\title{
NANO DREINFORGEMENTOF
}

TIRERUBBERS॰

SILCA DTECHNOLOGYFOR NATURALRUBBER

Explosing the Influence of

Von:Rubber Constituentson

the Watural Rubbers:Silica System 
NANO-REINFORCEMENT OF TIRE RUBBERS: SILICA-TECHNOLOGY FOR NATURAL RUBBER

Exploring the Influence of Non-Rubber Constituents on the Natural RubberSilica System 
The research described in this thesis was financially supported by the Malaysian Rubber Board (MRB).

\section{Graduation committee}

$\begin{array}{lll}\text { Chairman: } & \text { Prof. Dr. G.P.M.R. Dewulf } & \text { University of Twente } \\ \text { Secretary: } & \text { Prof. Dr. G.P.M.R. Dewulf } & \text { University of Twente, CTW } \\ \text { Promoter: } & \text { Prof. Dr. Ir. J.W.M. Noordermeer } & \text { University of Twente, CTW } \\ \text { Ass. Promotor: } & \text { Dr. Dipl-Ing. W.K. Dierkes } & \text { University of Twente, CTW } \\ \text { Members: } & \text { Prof. Dr. Ir. E. van der Heide } & \text { University of Twente, CTW } \\ & \text { Prof. Dr. Ir. J. Feijen } & \text { University of Twente, TNW } \\ & \text { Prof. Dr. N. Vennemann } & \text { University of Applied Sciences } \\ & & \text { Osnabrück, Germany } \\ \text { Referees: } & \text { Dr. Z. Mohd Nor } & \text { MRB, Malaysia } \\ & \text { Dr. S. Cook } & \text { Tun Abdul Razak Research Centre, } \\ & & \text { United Kingdom }\end{array}$

Nano-reinforcement of tire rubber: silica-technology for natural rubber.

Exploring the influence of non-rubber constituents on the natural rubber-silica system

By Siti Salina Sarkawi

Ph.D Thesis, University of Twente, Enschede, the Netherlands, 2013.

With references - With summary in English, Dutch and Malay.

Copy right (C) Siti Salina Sarkawi 2013.

All rights reserved.

Cover design by Mohd Fauzi Mohd Anuar

Cover illustration background: Hevea Brasiliensis leaf

Printed at Wöhrmann Print Service, Postbus 92, 7200 AB Zutphen, the Netherlands.

ISBN: 978-90-365-0071-5

DOI: $\quad 10.3990 . / 1.9789036500715$

USL: $\quad$ http://dx.doi.org/10.3990/1.9789036500715 


\section{NANO-REINFORCEMENT OF TIRE RUBBERS: SILICA-TECHNOLOGY FOR NATURAL RUBBER}

\section{EXPLORING THE INFLUENCE OF NON-RUBBER CONSTITUENTS ON THE NATURAL RUBBER-SILICA SYSTEM}

\section{DISSERTATION}

to obtain

the degree of doctor at the University of Twente,

on the authority of the rector magnificus,

Prof. Dr. H. Brinksma,

on account of the decision of the graduation committee,

to be publicly defended

on Thursday, October $17^{\text {th }}, 2013$ at $12: 45$

by

Siti Salina Sarkawi

born on December $17^{\text {th }}, 1976$

in Johor, Malaysia 
This dissertation has been approved by:

Promoter : : Prof. Dr. Ir. J.W.M. Noordermeer

Assistant Promoter : Dr. Dipl- Ing. W.K. Dierkes 
"People, We have created you all from a single man and a single woman, and made you into races and tribes so that you should get to know one another. In God's eyes, the most honoured of you are the ones most mindful of Him: God is All-Knowing, All-Aware."

Al-Hujurat 49:13 



\section{TABLE OF CONTENTS}

Chapter $1 \quad$ Introduction

Chapter 2 A Review on Silica Reinforcement in Natural Rubber

Chapter $3 \quad$ The Influence of Processing Temperature on Silica 55

Reinforced Natural Rubber

Chapter $4 \quad$ The Influence of Non-Rubber Constituents on Silica 75

Reinforced Natural Rubber

$\begin{array}{ll}\text { Chapter } 5 & \text { Elucidation of Filler-to-Filler and Filler-to-Rubber } \\ \text { Interactions in Silica Reinforced Natural Rubber by TEM }\end{array}$

93

Network Visualization

Chapter 6 A Morphological Study of Macro- and Micro-Dispersion

of Silica in Natural Rubber Vulcanizates

Chapter 7 Mechanistic Study into the Effect of Proteins on

Chapter 8 The Performance of Modified-Silica in Natural Rubber

and Deproteinized Natural Rubber Compounds

Chapter $9 \quad$ Dynamic and Mechanical Properties of Silica

Reinforced NR/IR and NR/BR Blends

Appendix A study on Reactions of TESPT and Rubber in

Summary and Outlook

Samenvatting

Ringkasan

Symbols and Abbreviations

Bibliography

Acknowledgements

Curriculum Vitae 

Chapter 1

Introduction:

Natural Rubber, An Eco-Friendly Elastomer 


\subsection{Natural Rubber As Renewable Resource}

Natural rubber is a strategic material and due to its outstanding properties of elasticity, resilience, low hysteresis, low heat build-up, flexibility at low temperatures, resistance to tearing, abrasion, impact and corrosion, facile adhesion to textiles and steel, as well as its insulating properties and ability to disperse heat, it cannot be replaced by synthetic rubbers in some important applications ${ }^{1}$. Major applications of natural rubber can be divided into four sections: tires $(75 \%)$, automotive products $(5 \%)$, non-automotive products $(10 \%)$ and miscellaneous applications such as health related products (10\%). About $90 \%$ of natural rubber is produced in Asia, particularly Thailand, Indonesia and Malaysia.

Natural rubber enjoys a lot of advantages over synthetic rubbers. Natural rubber is a source of renewable material and gives tremendous environmental benefits. A synthetic rubber plant consumes energy and produces carbon dioxide $\left(\mathrm{CO}_{2}\right)$ in the conversion of crude oil into elastomers, whereas the natural rubber tree converts $\mathrm{CO}_{2}$ and solar energy into an elastomer. The energy input for natural rubber production is about 15-16 GJ/tonne, while in contrast synthetic rubber production such as styrene butadiene rubber (SBR) requires about $130-156 \mathrm{GJ} /$ tonne $^{2}$. Natural rubber plantations act as a significant sink for $\mathrm{CO}_{2}$ produced through the combustion of fossil fuel. The high photosynthetic rate of a mature rubber (Hevea Brasiliensis) leaf is about $11 \mu \mathrm{molm}^{-2} \mathrm{~s}^{-1}$ as compared to $5-13$ $\mu \mathrm{molm} \mathrm{m}^{-2} \mathrm{~s}^{-1}$ in other tree species ${ }^{3}$, this makes it an efficient $\mathrm{CO}_{2}$ sequester. The amount of carbon sequestration per hectare by rubber tree is determined as 272 tonnes within a 30 year life period, in comparison with 234 tonnes per hectare by rain forest and 150 tonnes by secondary rain forest ${ }^{3 a}$. Moreover, the rubber trees after their service life are a valuable source of timber. In terms of the socio-economic importance of the rubber industry, about 40 million people are dependent directly or indirectly on natural rubber for their livelihood ${ }^{4}$.

The global natural rubber consumption has shown a steady increase during the last decade: Figure 1.1. Out of 25.9 million tonnes of world rubber consumption in $2012,42 \%$ are natural rubber ${ }^{5}$. Global natural rubber consumption in 2015 may grow by $6 \%$ to about 12.3 million tonnes, according to the International Rubber Study Group (IRSG) forecast ${ }^{6}$.

The fact that no other suitable alternative materials can replace rubber has the consequence that the amount of rubber and rubbery materials used per automotive vehicle since the 1970s till now has not changed significantly. The use of natural rubber in modern radial truck tires has risen as compared to equivalent bias tires ${ }^{7}$. For one radial truck tire, approximately $21 \mathrm{~kg}$ of natural rubber is required as compared to $9 \mathrm{~kg}$ in a bias truck tire.

\footnotetext{
${ }^{a}$ Secondary forest is rainforest that has been disturbed naturally or unnaturally, and characterized by a less developed canopy structure, smaller trees and less diversity. 


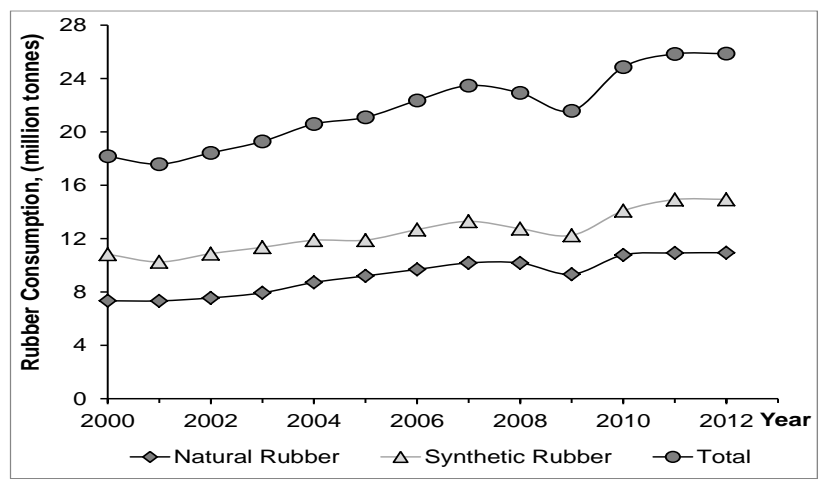

Figure 1.1 World rubber consumption

With an increasing demand for renewable materials flourishing from the growing concerns on climate change and global warming, natural rubber has gained a strong position as an eco-friendly elastomer and a strategic green material for rubber applications.

\subsection{Silica Technology and Motivation of the Project}

The use of silica has become of growing importance in tires, because of the need for reduced fuel consumption in automotive transport and consequently preservation of the environment. The high-dispersion silica reinforcement was introduced in the early 1990 by Michelin ${ }^{8}$ in passenger car tire-tread rubbers, the so-called "Green Tire", which offers approximately $30 \%$ lower rolling resistance in tires, resulting in $5 \%$ fuel savings, and consequently lower carbon dioxide emission to the environment.

Silica-technology encompasses four essential elements (Figure 1.2): the rubber polymer, a special type of silica, an effective coupling agent and the appropriate mixing technology, which form the basis for shifting the magic triangle of tire technology: the compromise between rolling resistance, (wet) traction and wear.

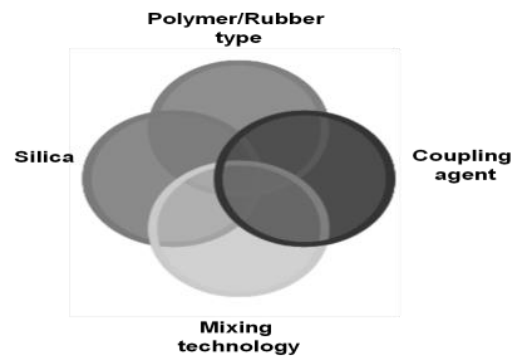

Figure 1.2 The elements of silica technology 
The high-dispersion silica technology, as it is used today, dictates the use of "solution-polymerized" synthetic elastomers e.g. solution Styrene-Butadiene Rubber (sSBR) and solution Butadiene Rubber (BR). On the other hand, the great majority of rubber polymers used for carbon-black reinforced tire applications are "emulsion-polymers". These emulsion-polymers are either synthetically produced in latex emulsion polymerization, like e.g. emulsion Styrene-Butadiene Rubber (eSBR), the most commonly used alternative for Natural Rubber (NR) in tires, or they are harvested in the form of a natural latex emulsion: NR.

The core of the high-dispersion silica technology is the nano-scale reaction of the 4-6 silanol-groups per $\mathrm{nm}^{2}$ on the surface of the 20-30 nm diameter primary silica-particles with a coupling agent. This reaction reduces the hydrophilic character of the filler and increases its compatibility with the rubber polymer. It is due to take place in the same processing step as mixing of the tire compound, and is very difficult to lead to completion. The coupling agent eventually creates a chemical link between the primary silica particles and the rubber molecules during the later processing step of vulcanization.

Up until now, the reason for not utilizing NR in silica technology and its ineffectiveness with silane coupling agents is still not fully understood. The non-rubber components, especially proteins are believed to be the origin of the outstanding rubber properties of NR. Nevertheless, it is postulated that the proteins contained in NR, or emulsifiers in eSBR, compete with the coupling agents for reaction with the silica during mixing, so disturb its reinforcement action and consequently affect the final properties of the compound.

The motivation of this study is therefore to develop the necessary means so as to make the use of NR more suited for high-dispersion silica reinforcement of tire-treads, by controlling NR composition and increasing the reactivity of NR towards silica. Therefore, an insight into the influence of non-rubber constituents in NR on silica-silica, silica-silane and silica-rubber interactions is required to further understand the mechanism of silica reinforcement in NR.

\subsection{Aim OF THE Project}

The research described in this thesis is aimed to gain insight in the silica reinforcing mechanism in NR compounds. The role of non-rubber constituents, particularly proteins on silica-silica and silica-silane interactions is investigated. The critical factor in silica technology is the nano-scale coupling between rubber molecules and the silica surface via 
coupling agents, not to be disturbed by other surface-active agents like proteins. The adsorption of proteins on silica surfaces will be controlled to improve the mixing of silica reinforced tire rubber, by the use of purified NR or deproteinized NR (DPNR), that has a controlled low protein content. A comparison is made with the absence of the non-rubber constituents by the use of the synthetic substitute of NR, polyisoprene (IR). A better understanding of the influence of non-rubber constituents, particularly proteins on the reaction between silica and silane coupling agents may lead to further developments for improving the reactivity of NR towards silica.

Another route to meet this challenge is pretreatment of silica with a coupling agent to minimize the adsorption of proteins on the silica surface, as well as blending NR with vinyl-containing synthetic rubbers to improve the reactivity of the coupling agent towards NR.

\subsection{CONCEPT OF THIS THESIS}

This thesis starts with an introduction of the advantages of NR as renewable resource, silica technology in tires as the background of this project and the aim of the project. In Chapter 2, a literature overview on the fundamental network structure of NR, non-rubber constituents in NR particularly proteins, and purification of NR is given. In addition, a comprehensive review on silica reinforcement in NR with emphasis on tire properties is presented.

This thesis encompasses 7 experimental chapters, which can be divided into two categories. The first five chapters are dedicated to investigate the influences of protein content in NR on silica reinforcement and the last two chapters are alternatives to address the challenges for improving silica reinforcement in NR.

Chapter 3 - The reinforcement of NR by precipitated silica as influenced by mixing temperature is studied in this chapter. A phenomenological investigation of the silica-silane system in NR is directed towards the absence of non-rubber constituents with DPNR and IR.

Chapter 4 - The effect of proteins on silica-silica and silica-silane interactions in the presence and absence of a silane coupling agent is investigated. Since proteins are complex materials, skim rubber with high protein content is considered as an option to represent the proteins in NR. A comparative study on silica reinforcement in three types of natural rubber with varying protein contents: NR, DPNR and skim rubber, is discussed.

Chapter 5 - The focus within this chapter is on filler-to-filler and filler-to-rubber interactions in silica-filled NR presented by the special technique of Transmission Electron Microscopy (TEM) Network Visualization. 
Chapter 6 - The macro- and micro-dispersion of silica in NR are further investigated using several different morphological techniques such as AFM, TEM, SEM and reflective light microscopy. The relation of silica-dispersion with the Payne effect or silicasilica interaction is elaborated.

Chapter 7 - A strong effect of proteins in hydrophobation of the silica surface is demonstrated with the use of proteins from NR serum in a silica-filled compound. The competitive interaction between proteins and silane on the silica surface is discussed. The mechanism of the adsorption of proteins on silica surface interaction is studied in detail with the use of a glass slide surface and compared to silica powder.

Chapters 8 - The use of pre-treated silica in NR and DPNR compounds is investigated in this chapter with the aim to circumvent the effect of non-rubber adsorption onto the silica surface. The reinforcing effect of pre-treated silica at varying amounts is studied. A comparison in properties of the in-situ TESPT-treated silica is also discussed.

Chapters 9 - In order to improve the efficiency of silica reinforcement in NR, blends of NR/IR and NR/BR are studied. Both, the IR and BR have 10\% vinyl-content which is aimed to improve the reactivity of silane towards rubber. Dynamic and mechanical properties of both, NR/IR and NR/BR blends, are compared at different blend ratios.

Summary - The overall conclusions of this thesis as well as suggestions for further study are given in this section.

\subsection{REFERENCES}

1. M.M. Rippel and F. Galembeck, Braz. Chem. Soc., 20 (6), 1024 (2009).

2. K.P. Jones, Kautsch. Gummi. Kunstst., 53, 735 (2000).

3. C.-M. Cheng, R.-S. Wang and J.-S. Jiang, J. Environ. Sci., 19, 348 (2007).

4. R. Mukhopadhyay, R. Sengupta and S.D. Gupta, "Recent Advances in Eco-Friendly Elastomer", in Current Topics in Elastomer Research, ed. A.K. Bhowmick, CRC Press, Boca Raton (2008).

5. Malaysian Rubber Board, Malaysian Rubber Statistic 2012.

6. Secretariat of the International Rubber Study Group, presented at international Smallholder Rubber Conference, Phnom Penh, Cambodia (2009).

7. B. Rodgers and W. Waddell, "The Science of Rubber Compounding", in: Science and Technology of Rubber, eds., J.E. Mark, B. Erman and F.R. Eirich, Elsevier Academic Press, USA, (2005).

8. R. Rauline, EU Patent 0501227A1, to Michelin \& Cie (1992). 


\section{Chapter 2}

\section{A Review on Silica Reinforcement in Natural Rubber}

This chapter gives an overview of the research on silica reinforcement in elastomers with emphasis on natural rubber (NR) and its importance in tire technology. The use of silica with and without silane coupling agents and consequent improvement in tire properties especially rolling resistance, wet grip and heat build up is discussed. Mixing silica into rubber compounds and the influence of additives on silica-filled rubber is also highlighted. Most of the early silica compound development involved NR as the base polymer. Silica has a positive influence on reduced rolling resistance ( $\tan \delta$ at $60^{\circ} \mathrm{C}$ ) and lower heat buildup ( $\left.\Delta T_{\text {centre}}\right)$ as compared to carbon black in $N R$ compounds. $A$ better understanding of the silica reinforcing mechanism in NR compounds will facilitate further development of silica technology for NR, especially for use in highly filled silica tire compounds. $^{a}$

\footnotetext{
${ }^{a}$ Part of this chapter is published in Malaysian Rubber Board monograph no.22 (2011)
} 


\subsection{Natural Rubber}

Natural rubber $(N R)$ is a linear, long chain polymer with repeating isoprene units ${ }^{1}\left(\mathrm{C}_{5} \mathrm{H}_{8}\right)$. It has a density of 0.915 at $20^{\circ} \mathrm{C}$ and glass transition temperature of $200.5 \mathrm{~K}$. The major component of NR is cis-1,4 polyisoprene, as shown in Figure 2.1.

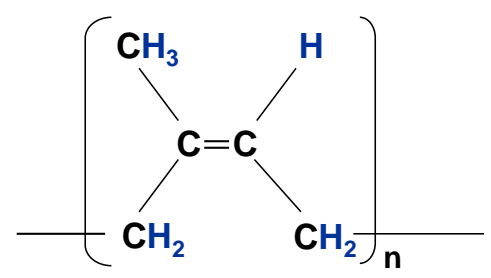

Figure 2.1 Natural rubber, cis-1,4 polyisoprene.

The commercial Natural rubber (NR) comes from the milky sap or latex that exudes from the rubber tree, Hevea Brasiliensis, which coagulates on exposure to air. Hevea latex is a complex emulsion of rubber hydrocarbon, proteins, lipids, phospholipids and water. There are many potential alternative sources of natural rubber which are being investigated, for example Guayule (Parthenium argentatum Gray), Russian dandelion (Taraxacum koksaghyz) and Canadian goldenrod (S. Canadensis) ${ }^{2}$.

There are three basic types of NR: technically specified rubber, visually inspected rubber and specialty rubber. The specification of technically specified NR is defined by the respective producing countries such as Standard Malaysian Rubber (SMR), Standard Indonesian Rubber (SIR) and Thai Technical Rubber (TTS) ${ }^{3}$. Examples of visually inspected natural rubber are ribbed smoked sheet (RSS), white and pale crepe and pure smoked blanket crepe. Specialty rubber includes liquid natural rubber (LNR), methyl methacrylate grafted rubber (MG rubber), deproteinized natural rubber (DPNR), epoxidized natural rubber (ENR) and superior-processing natural rubber.

The fundamental structure of NR has been revealed by NMR studies as a linear rubber chain consisting of $\omega$-terminal, two trans-1,4 isoprene units, long sequence of $1000-$ 3000 cis-1,4 isoprene units and $\alpha$-terminal ${ }^{4-6}$ as shown in Figure 2.2 The $\alpha$-terminal consists of mono- and diphospate groups linked with phospholipids by hydrogen bonds or ionic bonds ${ }^{7}$. The $\omega$-terminal is a modified dimethylallyl unit linked with functional groups, which is associated with proteins to form crosslinking via hydrogen bonding. These functional groups at both terminals are presumed to play a role in the branching and gel formation in $\mathrm{NR}^{8,9}$. 


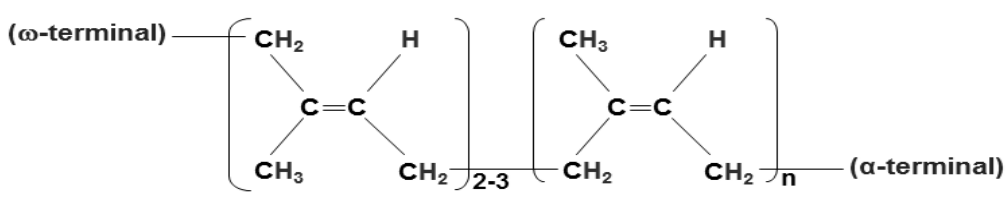

Figure 2.2 Fundamental structure of natural rubber ${ }^{5}$.

Natural rubber molecules exhibit some abnormal groups such as epoxide, carbonyl, and lactone ${ }^{4,6,10}$. Studies on NR characterization by Fourier transform infrared spectroscopy (FT-IR), ${ }^{13} \mathrm{C}-\mathrm{NMR}$, and ${ }^{1} \mathrm{H}-\mathrm{NMR}$ have shown that functional groups are present in the rubber molecules at the initiating and terminating ends ${ }^{10}$. These reactive groups (carbonyl and aldehyde) in the main chain of natural rubber molecules are assumed to be responsible for gel, branching formation and storage hardening in NR. Branching and crosslinking in NR are believed to come from the reaction of aldehyde and epoxide groups with amino acids of proteins (Figure 2.3).

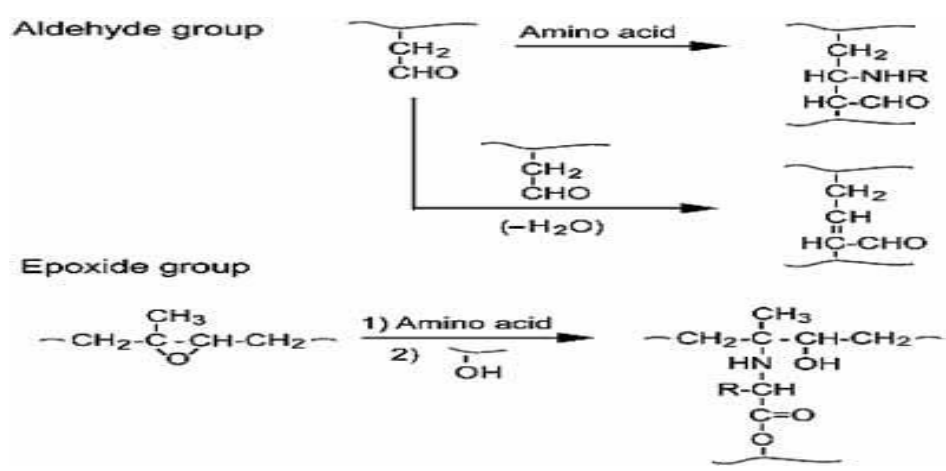

Figure 2.3 Functional groups in natural rubber that are associated with proteins to form crosslinking ${ }^{4}$.

A study on the effect of long chain branching in NR on carbon black mixing and incorporation time has shown that high branching provides a clear advantage and may enable more efficient mixing as well as better carbon black dispersion ${ }^{11}$.

\subsection{Proteins In Natural Rubber}

Hevea brasiliensis latex consists of rubber hydrocarbon, cis-1,4-polyisoprene, for about 3045 weight percent and non-rubber constituents for about $3-5$ weight $\%$, and the rest is 
water. The non-rubber constituents comprise of protein, amino acids, carbohydrates, lipids, amines, nucleic acids, as well as other inorganic and mineral components ${ }^{12}$. Some of these constituents are dissolved and lost in the aqueous serum during coagulation of NR latex.

Hevea latex can be separated using high speed centrifugation. After ultracentrifugation, the Hevea latex has mainly three basic fractions; a rubber phase, Cserum, and a bottom fraction as shown in Figure 2.4. C-serum contains a large variety of proteins and enzymes, whilst the bottom fraction contains mainly the lutoids, the source of B-serum, and other minor organelles ${ }^{13}$. B-serum is obtained via dialysis of the bottom fraction. Generally, the proteins from rubber particles and C-serum are acidic, whilst from Bserum comes a mixture of acidic and basic proteins. Most of the $\mathrm{C}$-serum and B-serum proteins are water soluble, however, the proteins from rubber particles are mainly nonsoluble in water. The distribution of protein in the centrifuged latex fraction is listed in Table $2.1^{14}$.

The non-rubber constituents that are retained in the rubber may influence the properties of rubber compounds, where proteins are believed to affect creep and heat buildup properties of vulcanizates, and amino acids affect the storage hardening of the raw rubber $^{12}$.

Midgley et al. have extracted protein constituents from natural rubber by hydrocarbon rubber removal with solvents, followed by electrodialysis of the residue ${ }^{15}$. The amino acids were identified as glycine, aspartic acid, leucine, proline, arginine, histidine, lysine and representative of the group comprising of alanine, phenylalanine, hydroxyproline and serine.

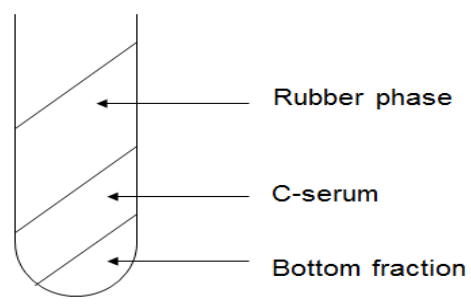

Figure 2.4 Hevea latex separate into three fractions after ultracentrifugation.

Table 2.1 Protein distribution in latex fraction

\begin{tabular}{lcc}
\hline Latex fraction & Protein concentration $(\mathrm{mg} / \mathrm{ml}$ latex $)$ & \% Distribution \\
\hline Rubber phase & 3.5 & 25 \\
C-serum & 6.0 & 43 \\
B-serum & 4.5 & 32 \\
\hline
\end{tabular}


The proteins in NR function as emulsifier in the rubber latex. These proteins are also responsible for dispersing the insoluble fraction of NR in a rubber solvent. The rubber end of the structure tends to dissolve in the rubber solvent, whilst the highly polar, insoluble protein end prevents solution.

Gregg and Macey ${ }^{16}$ have demonstrated that the insoluble non-rubber constituents in NR to a large extend account for the differences in properties between compounded natural rubber and compounded synthetic polyisoprene. This non-rubber material is mostly proteins and responsible for the higher modulus, faster scorch time and higher tear strength of NR. The protein is postulated to act as a reinforcing filler at low concentration (3-4 wt.\%) and as a cure activator. The amino acids identified in the proteins present in NR are listed in Table 2.2.

Table 2.2 Amino acid content of the denatured protein in natural rubber ${ }^{16}$

\begin{tabular}{|c|c|}
\hline Amino acid & Structure $(\mathrm{R})$ in $\mathrm{H}_{2} \mathrm{~N}-\mathrm{CH}(\mathrm{R}) \mathrm{COOH}$ \\
\hline Serine & $\mathrm{HO} \mathrm{CH} 2$ \\
\hline Glycine & $\mathrm{H}$ \\
\hline Leucine & $\left(\mathrm{CH}_{3}\right)_{2} \mathrm{CHCH}_{2}$ \\
\hline Alanine & $\mathrm{CH}_{3}$ \\
\hline Valine & $\left(\mathrm{CH}_{3}\right)_{2} \mathrm{CH}$ \\
\hline Glutamine & $\mathrm{H}_{2} \mathrm{NOCCH}_{2} \mathrm{CH}_{2}$ \\
\hline Lysine & $\mathrm{H}_{2} \mathrm{~N}\left(\mathrm{CH}_{2}\right)_{4}$ \\
\hline Arginine & $\mathrm{H}_{2} \mathrm{NC}(=\mathrm{NH}) \mathrm{NH}\left(\mathrm{CH}_{2}\right)_{3}$ \\
\hline Threonine & $\mathrm{CH}_{3} \mathrm{CH}(\mathrm{OH})$ \\
\hline Isoleucine & $\mathrm{CH}_{3} \mathrm{CH}_{2} \mathrm{CH}\left(\mathrm{CH}_{3}\right)$ \\
\hline Proline & $\mathrm{CH}_{2} \mathrm{CH}_{2} \mathrm{CH}_{2} \rightarrow \mathrm{NH}$ \\
\hline Tyrosine & $\mathrm{HOC}_{6} \mathrm{H}_{4} \mathrm{CH}_{2}$ \\
\hline Asparagine & $\mathrm{H}_{2} \mathrm{NOC} \mathrm{CH}$ \\
\hline Phenylalanine & $\mathrm{C}_{6} \mathrm{H}_{5} \mathrm{CH}_{2}$ \\
\hline Glutamic acid & $\mathrm{HOOCCH}_{2} \mathrm{CH}_{2}$ \\
\hline \multicolumn{2}{|l|}{ Tryptophane } \\
\hline Methionine & $\mathrm{CH}_{3} \mathrm{SCH}_{2} \mathrm{CH}_{2}$ \\
\hline
\end{tabular}


Othman and Hepburn have shown that the presence of proteins from B-, C-serum and proteolipids did not significantly affect the elastic modulus of a rubber vulcanizate. However, the presence of its hydrolyzed constituents, amino acids (ex. ethanolamine and arginine), gave a marked increase in the modulus of vulcanizates ${ }^{12}$.

Amnuaypornsri et al. have proposed a linear rubber chain structure with a naturally occurring network associated with proteins and phospholipids, as shown in Figure $2.5^{6}$. Both non-rubber constituents, i.e. proteins and phospholipids are presumed to form two types of branch points ${ }^{17}$. This network plays a significant role in strain-induced crystallization of unvulcanized and vulcanized NR.

The nitrogen content of NR is related to the protein level. Generally, it is accepted that a conversion factor for nitrogen to protein is $6.25^{18}$. The protein content of NR varies upon its source and methods of processing. The typical raw rubber has a nitrogen content in the range of $0.3-0.6 \%$, but rubber prepared from concentrated latex will usually have one of two nitrogen levels, the normal latex grades having generally lower levels than the dry rubbers, with values around $0.2 \%$, whilst the skim rubber, with its higher protein content, will have nitrogen values in the range of $1.5-2.5 \%$.

Skim rubber is a by-product of latex concentrate production and has a high protein content ${ }^{19}$. During latex concentrate production, after centrifugation of $5-10 \%$ of the total rubber, many of the non-rubber constituents remain in the serum phase. This is coagulated with sulfuric acid to produce rubber with a low dirt content and light color and that is relatively cheap. Due to the high proportion of protein in skim rubber, it is fast curing, giving vulcanizates of high modulus

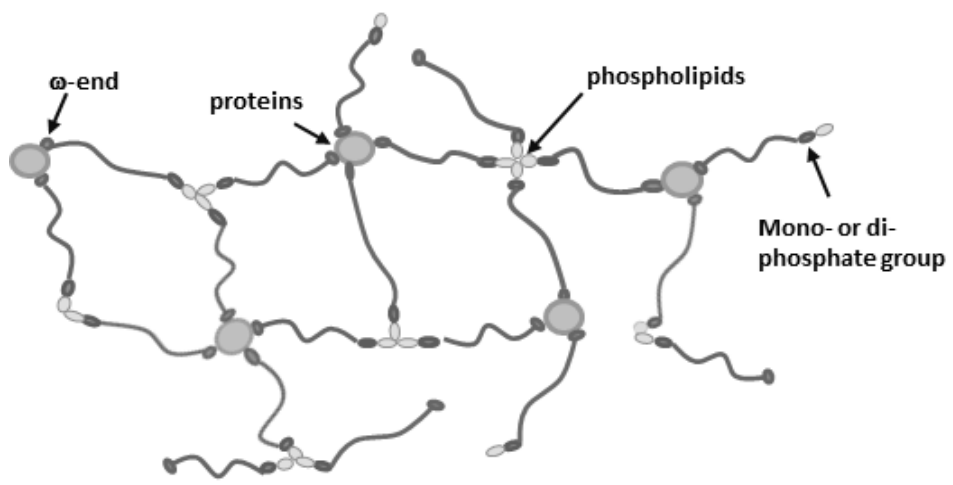

Figure 2.5 Linear rubber chain structure with the naturally occurring network associated with proteins and phospholipids ${ }^{6}$. 


\subsection{Purified Natural Rubber}

Many attempts have been made to purify natural rubber from the non-rubber constituents such as proteins. One of the most successful attempts is the 'Deproteinized Natural Rubber' (DPNR) which is characterized by its very low nitrogen, ash and volatile matter contents compared to the equivalent properties of commercial natural rubber.

DPNR is produced via treatment of natural rubber latex with a bioenzyme (proteinase), which hydrolyzes the proteins present into water soluble forms ${ }^{1}$. A protease produced by non-pathogenic microorganism like Bacillus subtilis is used at 0.3 parts per hundred rubber (phr). The enzymolysis process takes a minimum of 24 hours. After completion, the latex is diluted to $3 \%$ total solids content and coagulated by adding a mixture of phosphoric and sulfuric acids. The coagulated rubber is then pressed, crumbed, dried and baled.

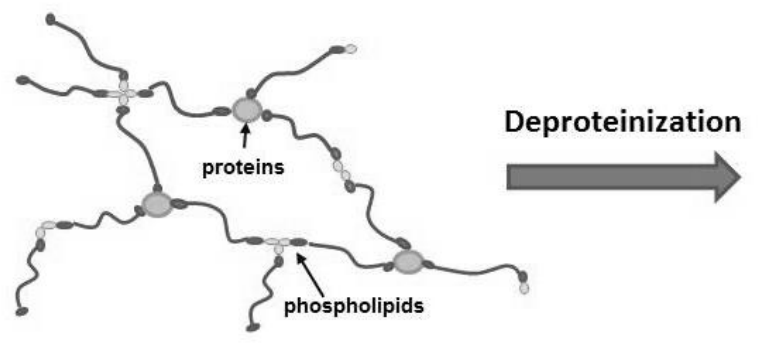

NR

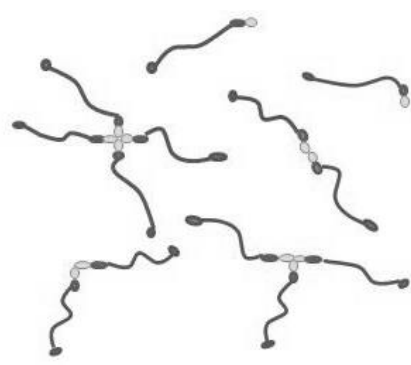

DPNR

Figure 2.6 Structural changes of branch points in NR after deproteinization.

Tanaka and coworkers have proposed the structural changes of branch points that occur with the deproteinization process as given in Figure 2.6. This is based on the findings that a linear rubber chain contains two types of functional groups at both terminals. After deproteinization, the branch points formed by the functional groups associated with proteins at the $\omega$-terminal through hydrogen bonding decompose, and leave the branch points from phospholipid at the $\alpha$-terminal. The findings by Amnuaypornsri showed that long-chain branching in the purified NR originated from the interaction of phospholipids which link the rubber chains together (Figure 2.7) ${ }^{20-22}$. The phospholipids are associated together by the formation of a micelle structure. 


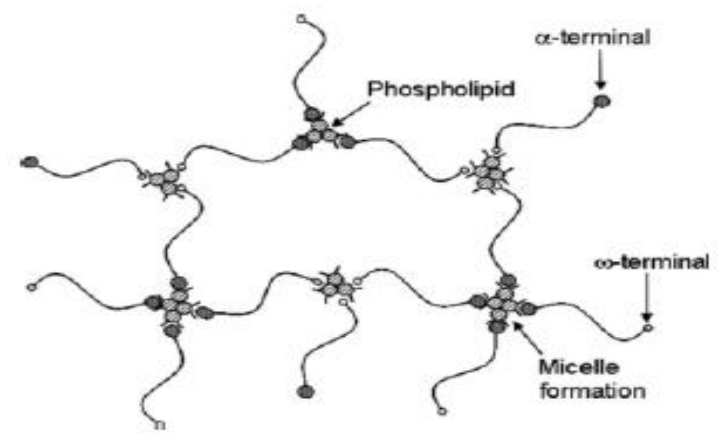

Figure 2.7 Proposed structure of branching and gel formation in DPNR ${ }^{20}$.

The commercially available DPNR is Pureprena, which is produced by the Malaysian Rubber Board. Pureprena is a purified form of natural rubber and has a very low nitrogen, ash and volatile matter contents as well as being lighter in color (Table 2.3). When compounded using an efficient vulcanization (EV) system, DPNR has low creep and stress relaxation, low water absorption, low compression set and a more consistent modulus when subjected to variable humidity conditions ${ }^{23}$. DPNR gives superior rubber compounds with excellent dynamic properties which are suitable for engineering applications.

Table 2.3 The specification and typical raw rubber properties of DPNR (Pureprena)

\begin{tabular}{|c|c|c|}
\hline Properties & $\begin{array}{c}\text { Specification of } \\
\text { Pureprena }\end{array}$ & $\begin{array}{c}\text { Typical properties of } \\
\text { Pureprena }\end{array}$ \\
\hline Dirt retained on $44 \mu \mathrm{m}$ aperture (\% wt) & $0.01 \max$ & 0.003 \\
\hline Ash content, (\% wt) & $0.15 \max$ & 0.09 \\
\hline Nitrogen content, (\% wt) & $0.12 \max$ & 0.08 \\
\hline Volatile matter content, (\% wt) & $0.30 \max$ & 0.17 \\
\hline
\end{tabular}

\subsection{EMULSION- vS. SOLUTION-POLYMERIZEd RUBBERS}

Both polymer macrostructure and microstructure are important in determining the rubber characteristics required to meet tire performance properties ${ }^{3}$.

Polymer macrostructure: molecular weight, crosslink distribution, chain branching, crystallite formation

Polymer microstructure: the arrangement of the monomers within a polymer chain (molecular configurations, stereochemistry) 
For example, butadiene rubber (BR) can adopt one of three configurations as base units: units with cis or trans internal double bonds from 1,4 addition and units with side vinyl groups from 1,2 addition. BR with high cis content (92\%) are more difficult to process at factory processing temperature but show better abrasion resistance. High-trans BR $(93 \%$ trans) tends to be tough, crystalline materials, whereas high-vinyl BR shows good wet skid and wet traction performance.

A study on comparison of the use of emulsion- and solution-polymerized SBR in tire performance was conducted by Brantley and Day ${ }^{24}$. Solution-SBR had a narrower molecular weight distribution and lower $\mathrm{T}_{\mathrm{g}}$ than emulsion-SBR. This lead to lower hysteretic properties for solution-SBR. The authors also noted that a solution-SBR with the same bound styrene as an emulsion-SBR will give lower rolling resistance, improved dry traction and better tread wear. Nonetheless, emulsion-SBR shows better wet skid, wet traction and wet handling performance.

Kern and Futamura have concluded that the number-average molecular weight, $M_{n}$ is considered as key parameter of polymer macrostructure, with respect to hysteretic characteristics of a tread compound ${ }^{25}$. The molecular weight of solution-SBR is much higher than emulsion-SBR, where $M_{n}$ of emulsion-SBR is typically 90,000 to 175,000 , whilst $M_{n}$ of solution-SBR is 250,000 . Emulsion-SBR contains about $92 \%$ rubber hydrocarbon as a result of the presence of residues from the production process such as emulsifiers. In contrast, solution-SBR has near to $100 \%$ rubber hydrocarbon. Hence, the difference in the macrostructure between emulsion- and solution-SBR will eventually dictate the many differences in their properties in tire tread compounds (Table 2.4).

Table 2.4 Comparison of emulsion and solution-polymerized $\mathrm{SBR}^{25}$

\begin{tabular}{lcc}
\hline Properties & Emulsion-SBR & Solution-SBR \\
\hline Viscosity (ML[1+4] at $100^{\circ} \mathrm{C}$ ) & 50 & 57 \\
Optimum cure time at $150^{\circ} \mathrm{C}$ (minutes) & 40 & 25 \\
Tensile strength (MPa) & 26 & 21 \\
Elongation at break (\%) & 400 & 300 \\
Rebound resilience (\%) & 48 & 61 \\
\hline
\end{tabular}

Polymer microstructure will play a greater role when considering only solution polymers. Table 2.5 shows the effect on tire traction, rolling resistance and tread wear when the vinyl-1,2-butadiene level in BR is increased from 10 to $50 \%{ }^{24}$. 
Table 2.5 Effect of vinyl level in BR on tire performance properties ${ }^{24}$

\begin{tabular}{lcc}
\hline Tire properties & 10\% Vinyl content & 50\% Vinyl content \\
\hline Glass transition temperature $(\stackrel{\circ}{ } \mathrm{C})$ & -90 & -50 \\
Wet traction rating (100\%) & 100 & 120 \\
Rolling resistance rating (100\%) & 100 & 95 \\
Tread wear rating (100\%) & 100 & 90 \\
\hline
\end{tabular}

\subsection{SiLica As Reinforcing Filler}

Silica is a strongly polar and hydrophilic reinforcing filler. The characteristic structure of silica can be divided into agglomerates, aggregates and primary particles: Figure 2.8. The agglomerate of silica is typically in the dimension of $1-40 \mu \mathrm{m}$. This agglomerate of silica is formed by the agglomeration or network structure of silica aggregates by hydrogen bonding and Van der Waals forces. The typical dimensions of the silica aggregates are 100-500 nm. The silica aggregates are formed by the reaction of primary particles during the dehydration. Within the aggregates, the nano-size primary particles are linked together via siloxane bonds. The size of the primary particle is between $5-45 \mathrm{~nm}$.

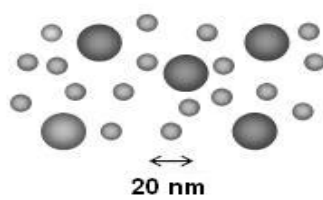

Primary particles Isolated

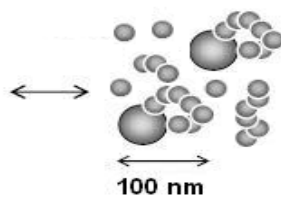

Aggregates Si-O-Si bonds

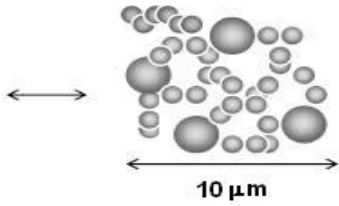

Agglomerates

Van-der-Waals H-bridges

Figure 2.8 The characteristic structure of silica.

The silica surface is composed of siloxane and silanol groups (Figure 2.9 $)^{26}$. The silanol groups present on the surface of silica can be divided into three different types; depending on the hydroxyl group, which is;

- Isolated silanol group : a single hydroxyl group on a silicon atom

- Vicinal silanol group : two hydroxyl groups on adjacent silicon atoms

- Geminal silanol group : two hydroxyl groups on the same silicon atom

In addition, a siloxane bridge is formed when one oxygen atom is shared by two silicon atoms as shown in Figure 2.9. The silanol groups are detectable by Infrared (IR) spectroscopy as presented in Figure $2.10^{27}$ as well as in Table $2.6^{26}$. 


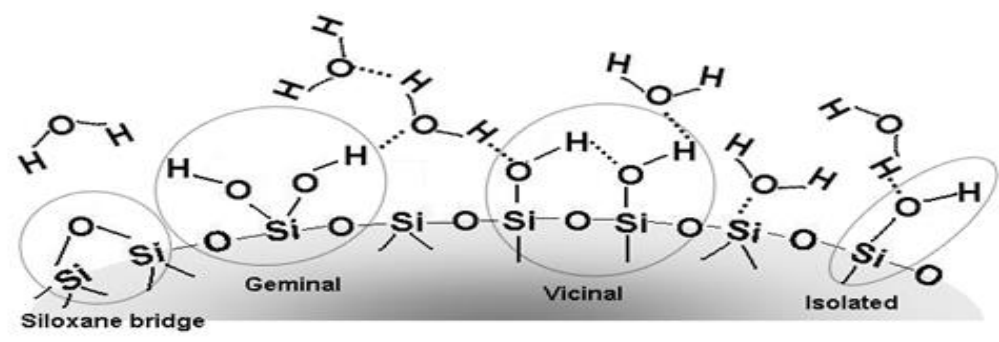

Figure 2.9 The siloxane and silonal groups present on a silica surface.

The reinforcement of rubber by silica is different from carbon black. The use of conventional silica has been limited as white filler for colored rubber compounds ${ }^{26}$ such as shoe soles, until the introduction of bifunctional silanes as coupling agent back in 1970 's ${ }^{28}$. Silica has been employed in small quantities together with carbon black to improve the traction of truck tire treads. Only recently, a full replacement of carbon black with silica is possible and used as reinforcement in passenger tire-treads. This is derived from the breakthrough in tire innovation with the introduction of the "Green Tire" by Michelin with a tread based on silica from Rhodia and silane from Degussa ${ }^{26}$. The specific silanol group density and the surface activity are important parameters for the improvement of the dispersion behavior, the dynamic stiffness and cure rate of a silica-filled compound ${ }^{29}$.

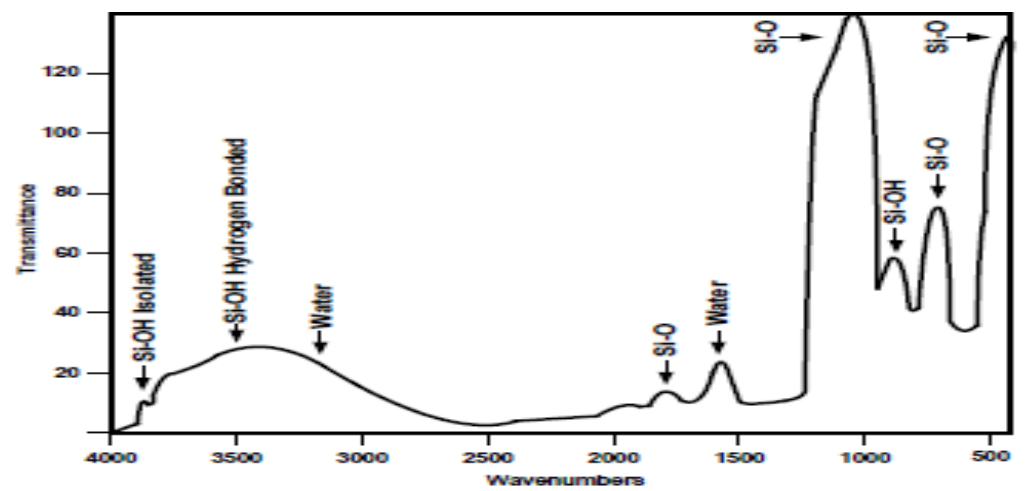

Figure 2.10 IR Spectrum of silica ${ }^{27}$.

Easy incorporation of silica into a rubber mixture and good dispersion of silica are important parameters as they affect the processing and compound properties. Silica can be classified according to their ease of dispersion into three categories; namely conventional, 
easily dispersible or semi-HD, and highly dispersible (HD) silicas. Table 2.7 lists various commercial silicas as classified according to surface area and ease of dispersion. A higher dynamic mechanical tan $\delta$ at $0^{\circ} \mathrm{C}$ and a lower tan $\delta$ at $60^{\circ} \mathrm{C}$, which indicate improved traction and lower rolling resistance respectively, were obtained with silica-filled compounds containing HD silica with high surface area and uniformly small pore diameter, compared to compounds with conventional silica or carbon black ${ }^{30}$.

Table 2.6 Silanol groups detectable by IR spectroscopy

\begin{tabular}{lc}
\hline & Infrared band $\left(\mathbf{c m}^{-1}\right)$ \\
\hline Isolated & $3740-3750$ \\
Vicinal & $3640-3660$ \\
Geminal & 3500 \\
Adsorbed water & $3420,3456,3480$ \\
\hline
\end{tabular}

Table 2.7 Classification of various commercial silica's

\begin{tabular}{|c|c|c|c|}
\hline $\begin{array}{l}\text { CTAB Surface } \\
\text { Area }\left(\mathrm{m}^{2} / \mathrm{g}\right)\end{array}$ & Conventional & Semi-HD & HD \\
\hline \multirow[t]{6}{*}{$100 \pm 20$} & Ultrasil 360 (GR) & Ultra VN2 (GR) & Zeosil $1115 \mathrm{MP}$ \\
\hline & Ultrasil AS 7, Ultrasil 880 & Zeosil $115 \mathrm{Gr}$ & Zeopol 8715 \\
\hline & Hubersil 1613, 1633, 1635 & Zeosil $1135 \mathrm{MP}$ & \\
\hline & Hi-Sil 315 & & \\
\hline & Zeosil 125 GR & & \\
\hline & Zeolex 23, 80 & & \\
\hline \multirow[t]{7}{*}{$160 \pm 20$} & Ultrasil VN3 & Ultrasil 3370 GR & Ultrasil7000 GR \\
\hline & Hubersil 1714, 1715, 1743 & Hi-Sil 243 MG & Zeosil $1165 \mathrm{MP}$ \\
\hline & Hubersil 1745 & Hi-Sil EZ & Zeopol 8745 \\
\hline & Hi-Sil 170, 210, 233, 255 & Huberpol 135 & Zeopol 8755 \\
\hline & Hi-Sil 243 LD & Zeosil 145MP & \\
\hline & Zeosil 145 GR, Zeosil 174 G & Zeosil 165 GR & \\
\hline & Zeolex 25 & & \\
\hline \multirow[t]{3}{*}{$200 \pm 20$} & Hi-Sil 170 & Hi-Sil $190 \mathrm{G}$ & Ultrasil 7005 \\
\hline & Hi-Sil 185/195 & Zeosil 195 MP & Zeosil $1205 \mathrm{MP}$ \\
\hline & Zeosil 195 GR & Zeosil 215 GR & Hi-Sil 2000 \\
\hline
\end{tabular}


The silica surface covered with a large number of silanol and siloxane groups can be characterized by the surface energy. The surface free energy of filler particles such as silica can be quantified using Inverse Gas Chromatography (IGC) ${ }^{31}$. The surface free energy of a solid or filler, $\gamma_{s}$, can be represented $a s^{31}$ :

$$
\gamma_{s}=\gamma_{s}^{d}+\gamma_{s}^{s p}
$$

Where, $\gamma_{s}^{d}$ is the dispersive component, and $\gamma_{s}^{s p}$ is the specific component of the surface free energy. Specific interactions measure the filler-filler interaction, which include hydrogen bonding, polar and acid-base interactions. The dispersive interactions between rubber and filler are non-specific interactions like van der Waals interactions.

In comparison with carbon black, the surface energies of silica are characterized by a lower dispersive component, $\gamma_{s}^{d}$ and a higher specific component, $\gamma_{s}^{s p 32,33}$. The low $\gamma_{s}^{d}$ of silica would result in low filler to rubber interaction, whilst high $\gamma_{s}^{s p}$ of silica leads to strong agglomeration of silica in the rubber matrix. In contrast, the high $\gamma_{s}^{d}$ of carbon black gives strong filler to rubber interaction.

\subsection{Silane Coupling Agents}

An organofunctional silane ${ }^{26,34}$ is a unique chemical that has vast applications. Due to its unique combination of organic activity and silicon reactivity, it is used in applications such as coatings, adhesives, sealants, elastomers, electronic materials, fiberglass, and foundry sand binders as well as in other many advanced and innovative technologies. A bifunctional organosilane coupling agent, through the organo-functional and silicon-functional moiety, is able to chemically bond a polymer matrix to inorganic substrates such as silica. The bifunctional organosilane coupling agent serves two functions; its one end is for coupling with the hydrophilic silica surface and the other end to couple with the hydrophobic polymer or rubber. Hence, the coupling agent acts as a connecting bridge between silica and the rubber and improves the reinforcement of silica in rubber.

The chemical structure of a bifunctional organosilane coupling agent is simply described as ${ }^{26,28,34-37}$ :

$$
\mathrm{X}_{3}-\mathrm{Si}-\left(\mathrm{CH}_{2}\right)_{\mathrm{n}}-\mathrm{Y}
$$

Figure 2.11 Structure of bifunctional silane coupling agent

where:

$X$ is the Silicon-functional group or hydrolysable groups which react with inorganic surfaces. The group may be halides, alkoxides or acyloxy. Some examples of this group include:

$$
-\mathrm{OCH}_{3},-\mathrm{OC}_{2} \mathrm{H}_{5},-\mathrm{OC}_{2} \mathrm{H}_{4} \mathrm{OCH}_{3}
$$


$\mathrm{Y}$ is the Organofunctional group that provides bonding with a polymer matrix, which can consist of an amino, methacrylate, epoxy, mercapto, glycidoxy, or cloropropyl group. Some examples of this group include:

$$
-\mathrm{SH},-\mathrm{NH}_{2},-\mathrm{Cl},-\mathrm{CH}=\mathrm{CH}_{2},-\mathrm{OC}(=\mathrm{O})-\mathrm{C}\left(\mathrm{CH}_{3}\right)=\mathrm{CH}_{2},-\mathrm{N}=\mathrm{C}=\mathrm{O} \text {, etc. }
$$

Stable $\left(\mathrm{CH}_{2}\right)_{n}$ carbon atoms are attaching the $\mathrm{Y}$ organofunctional group with the central silicon atom.

Organofunctional silane coupling agents chemically bond to organic polymers via different methods as follows ${ }^{34}$ :

- Reaction with terminal or pendant groups;

- this reaction can occur for isocyanato, hydroxyl and amine end blocked polymers as well as polymers with residual activated unsaturation.

- $\quad$ Grafting to reactive sites on the backbones;

- this method involves the free radical grafting with unsaturated silanes.

- Addition or condensation copolymerization;

- this method includes the incorporation within sulfur-crosslinked rubber, free radical-cured acrylates/methacrylates, thermoset epoxy resins, phenolic resins, and thermoset acrylic resins.

- Formation of interpenetrating polymer networks;

- silanes are used to form interpenetrating polymer networks.

The hydrolysable groups on silicon are able to react through hydrolysis and condensation. Alkoxysilane groups of silane coupling agents may react directly with a silanol group on the siliceous surface ${ }^{37}$, although a catalyst is recommended to accelerate the condensation.

Organofunctional silanes used for sulfur-cured rubber compounds can be categorized into the following three types ${ }^{26}$ :

$\begin{array}{lll}\text { Di- and polysulfide silanes : } & {\left[(\mathrm{RO})_{3}-\mathrm{Si}-\left(\mathrm{CH}_{2}\right)_{3}-\mathrm{S}_{2}-\mathrm{S}_{x}\right.} \\ \text { Mercaptosilanes } & (\mathrm{RO})_{3}-\mathrm{Si}-\left(\mathrm{CH}_{2}\right)_{3}-\mathrm{SH} \\ \text { Blocked Mercaptosilanes } & : & (\mathrm{RO})_{3}-\mathrm{Si}-\left(\mathrm{CH}_{2}\right)_{3}-\mathrm{S}-\mathrm{B} \\ \text { Where } \mathrm{R}=\mathrm{CH}_{3} \text { or } \mathrm{C}_{2} \mathrm{H}_{5} ; \mathrm{B}=\mathrm{CN} \text { or } \mathrm{C}_{7} \mathrm{H}_{15} \mathrm{C}=\mathrm{O} ; \quad x=0-8\end{array}$

Coupling agents may be premixed or pre-reacted with the silica filler or added to the rubber mix during the rubber and silica mixing stage. If the coupling agent and silica are added separately to the rubber mix during mixing, it is considered that the coupling agent then combines in situ with the silica ${ }^{38}$. 
To date, the common and effectively used silane coupling agents in rubber systems are bis(triethoxysilylpropyl) tetrasulfide (TESPT) ${ }^{39}$ and bis(triethoxy silylpropyl) disulfide (TESPD) ${ }^{26}$. The structure of TESPT or often called Si-69 is shown in Figure 2.12. TESPT is a silane with different sulfur ranks, ranging from $S_{1}$ to $S_{6}$ and average sulfur rank around 3.83 .

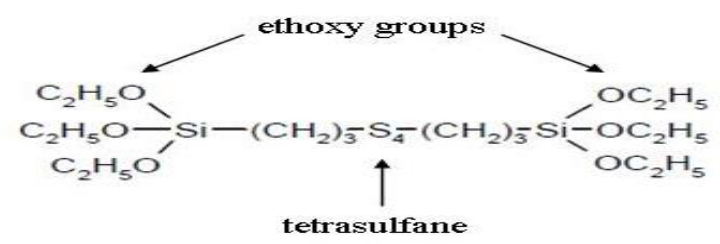

Figure 2.12 Bis(triethoxysilylpropyl) tetrasulfide (TESPT).

During mixing of a silica-filled rubber compound, the silane coupling agent reacts with the silica resulting in hydrophobation of the silica surface. This hydrophobation reduces the silica-silica network and makes the polar silica more compatible with the unpolar rubber.

The effect of silane coating on the surface free energy of silica has been assessed with $\mathrm{IGC}^{33}$. With silane modification, the surface chemistry of silica changes and both the dispersive and specific components of the silica surface free energy are reduced. This results in lower interaction between the silica particles and gives better dispersion of silica in a rubber matrix. The lower dispersive component on the surface energy of silane modified silica is compensated by introducing covalent linkages during vulcanization when bifunctional silanes are employed.

The reaction mechanism between silica, silane coupling agent and rubber has been extensively reviewed ${ }^{40-44}$. On one side, the triethoxysilyl group of the TESPT reacts with the silanol groups of silica during compounding with loss of ethanol. On the other side, the rubber reactive group of the silane (e.g. tetrasulfane) has a strong tendency to form rubber-to-filler bonds during curing of the rubber compounds. The primary and secondary reaction of TESPT and silica, as well as reaction of TESPT and rubber are shown in Figures $2.13,2.14$ and 2.15 , respectively.

The reactivity of a silane coupling agent is generally influenced by the hydrolysable group of the silane. By comparing the methoxy, ethoxy, propoxy and butoxy derivatives, the rate of silanization reaction decreases in the order ${ }^{44}$ :

$$
\mathrm{CH}_{3} \mathrm{O}->\mathrm{C}_{2} \mathrm{H}_{5} \mathrm{O}->\mathrm{C}_{3} \mathrm{H}_{7} \mathrm{O}->\mathrm{C}_{4} \mathrm{H}_{9} \mathrm{O}-
$$




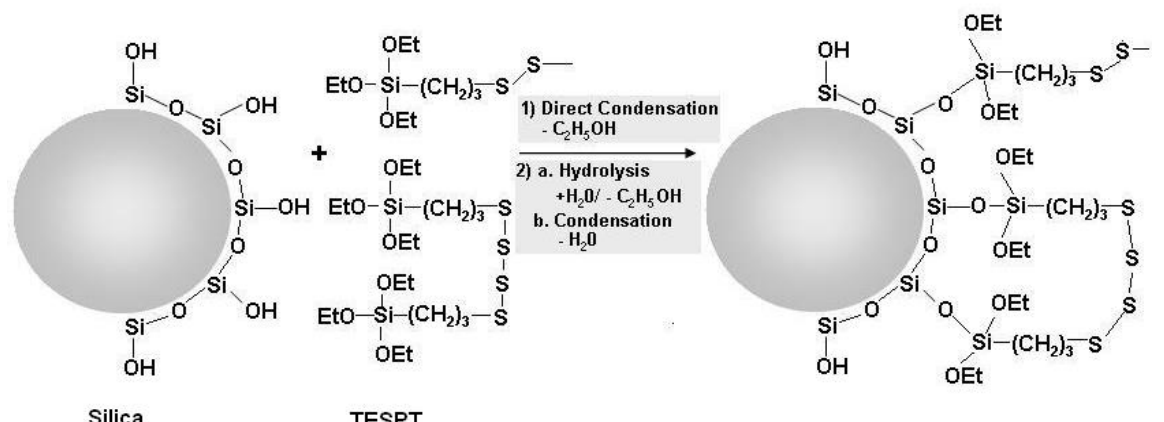

Figure 2.13 Primary reaction between silica and TESPT.
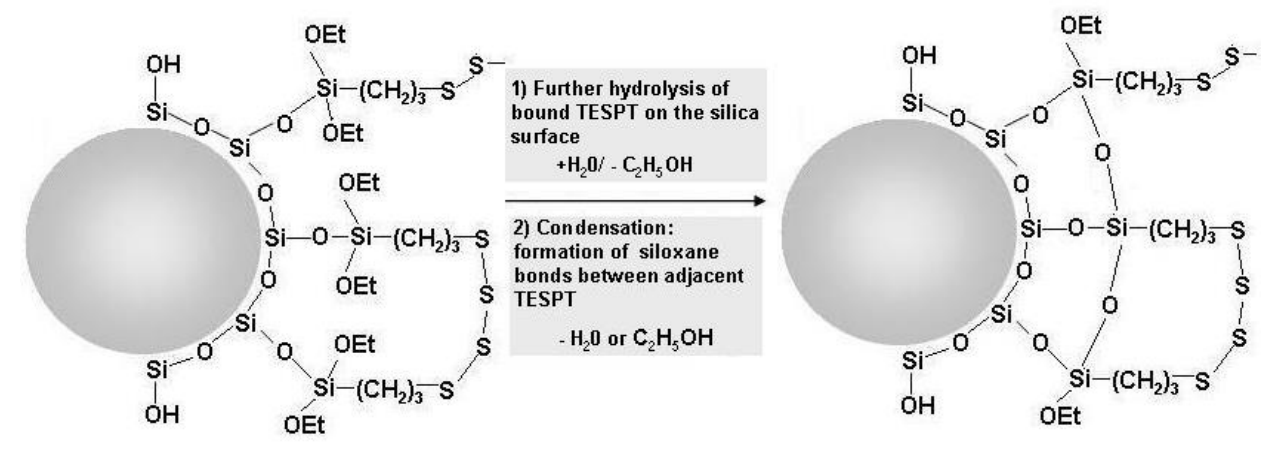

Figure 2.14 Secondary reaction between silica and TESPT.

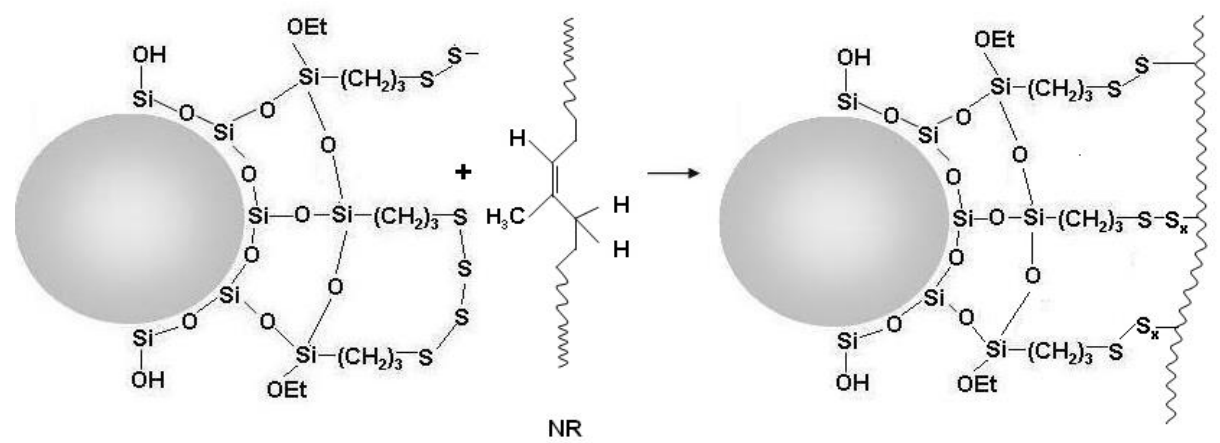

Figure 2.15 Reaction between rubber and TESPT. 
The reaction rate with propoxy and butoxy groups is too slow to be acceptable. The methoxy group reacts too rapidly, and it is not used as a silanization agent for toxicological reasons as it evolves methanol. Thus the ethoxy group is preferable as silanization agent, which reacts quickly enough and when precautions are taken, it is toxicologically harmless.

TESPT has been proven as curing agent and the carrier of the crosslinking reaction is the tetrasulfidic group ${ }^{45}$. At elevated temperature TESPT tends to disproportionate into bis-(3-triethoxysilylpropyl)-disulfide and bis-(3-triethoxysilyl propyl)polysulfides (Figure 2.16) where the sulfur chain has a mixture of less or more sulfur atoms than the tetrasulfide. With rubber, TESPT reacts as a sulfur donor which builds up rubber-torubber bonds (Figure 2.17). Nonetheless, the reaction of TESPT in silica-filled rubber compounds results in the immobilized tetrasulfidic group of the silane modified silica, which leads to formation of filler-to-rubber bonds (Figure 2.18).
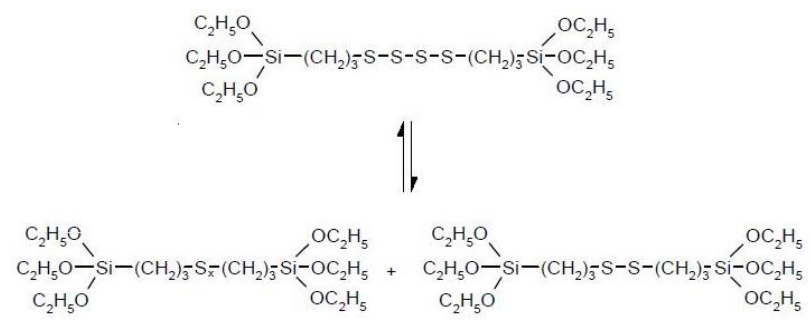

Figure 2.16 Disproportionation of TESPT ${ }^{45}$.

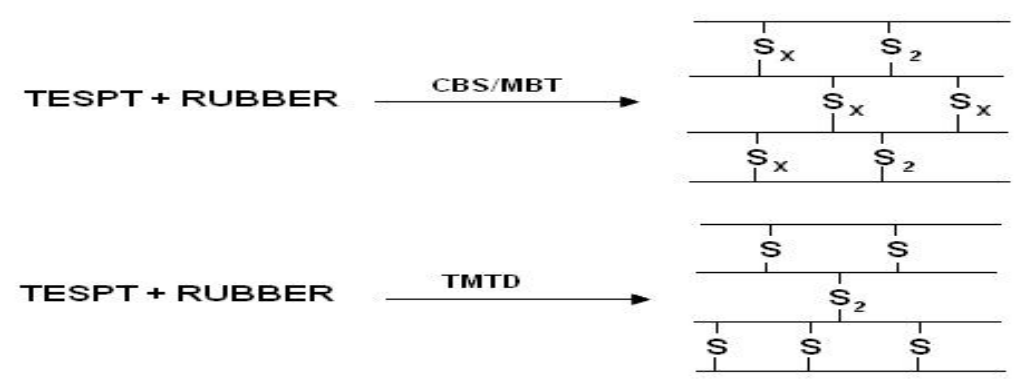

Figure 2.17 Types of rubber-to-rubber bonds built up with TESPT ${ }^{45}$. 


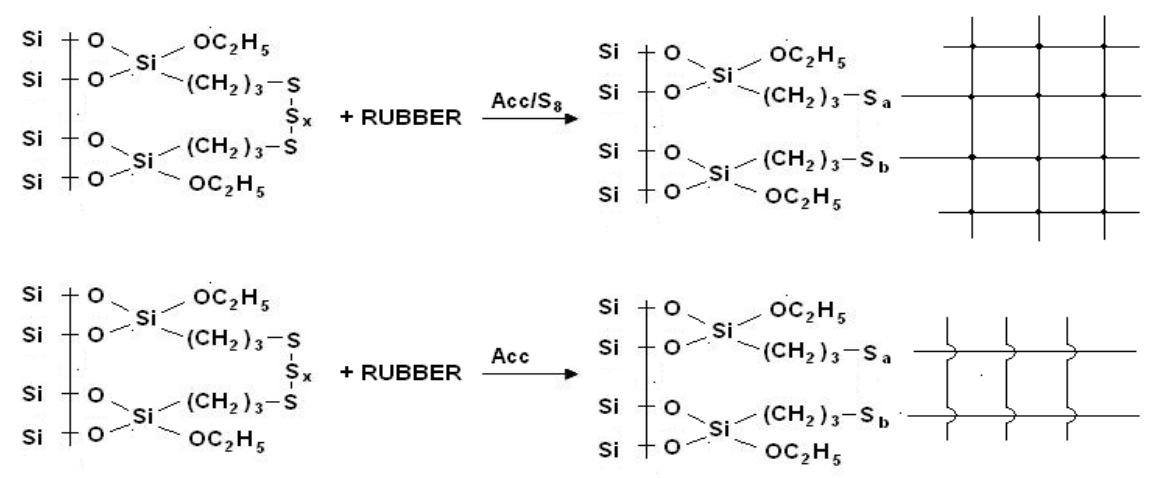

Figure 2.18 Build-up of filler-to-rubber bonds ${ }^{45}$.

\subsection{DeVelopment of Silica Technology in TiRe Rubbers}

When precipitated silica went into commercial production in 1948, NR was still the principal elastomer in commercial use. Hence, most of the early work on silica compound development involved NR as base polymer. It was evidenced that the use of silica in NR compounds attributed to tear strength, heat resistance and adhesion to fabrics and metal ${ }^{27}$.

\subsubsection{The USE of Silica Without Coupling Agent}

In their investigation on silica surface energies and interactions with model compounds, Wang et $a l^{31}$ have classified various rubbers with regard to their interaction with silica, based on thermodynamic adsorption parameters as follows:

$\mathrm{NBR}>\mathrm{SBR}>\mathrm{NR} \geq \mathrm{BR}>\mathrm{HV}-\mathrm{BR}>\mathrm{EPDM}>\mathrm{IIR}$

Where NBR is nitrile rubber, HV-BR is high-vinyl butadiene rubber, EPDM is ethylene propylene diene rubber and IIR is halogenated butyl rubber.

This compatibility chart between rubber and silica corresponds well with the solubility parameter of each rubber polymer ${ }^{43}$. Wang et al. concluded that aromatic hydrocarbons exhibit stronger interactions with silica than olefins ${ }^{31}$. Nonetheless, nitrile groups show the highest interaction with silica due to strong dipole-dipole interaction with polar surfaces as well as hydrogen-bonding interaction between the - $\mathrm{CN}$ group and silanol groups.

Tan and co-workers ${ }^{46}$ have shown that the low interaction of silica with nonpolar polymers and strong silica-silica interaction via hydrogen bonding resulted in marked flocculation between the silica aggregates, which caused high viscosity and high hardness. As depicted in Figure 2.19(a) there is sharp increase in Mooney viscosity for NR at high loading of silica as compared to NBR. This is primarily due to the stronger filler-filler interaction of silica in NR. 
The dynamic storage modulus, E', measured at room temperature as a function of filler volume fraction for NR and NBR is shown in Figure 2.19(b). Silica compounds show higher E', attributed to the stronger interaggregate interactions of silica than carbon black compounds. They also show higher filler-filler interactions for silica in NR as compared to NBR and this silica network formation provides a greater contribution to the E'.
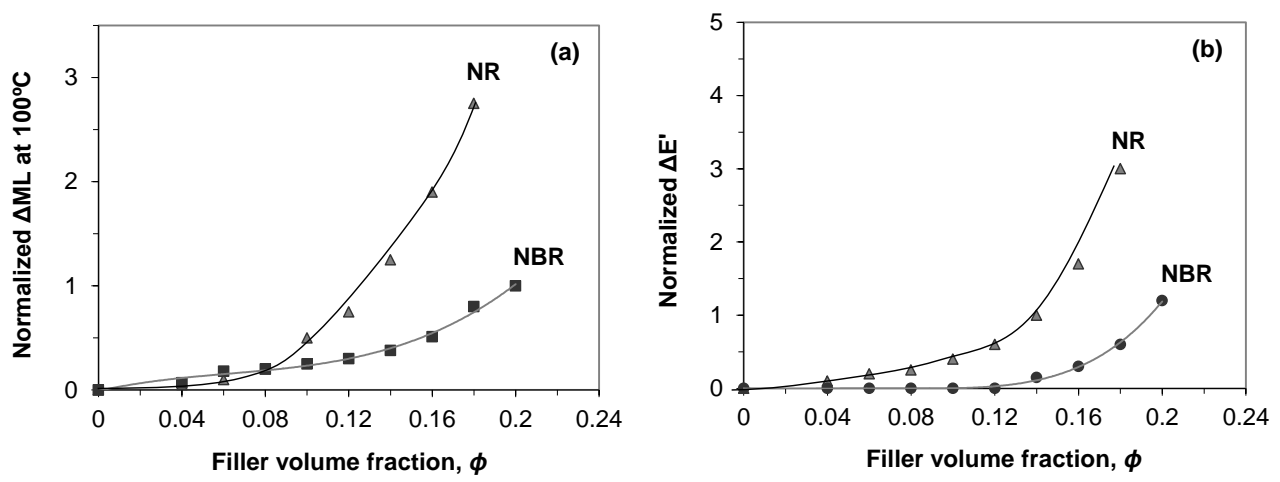

Figure 2.19 (a) Normalized Mooney viscosity; and (b) Normalized E', in NBR and $N R$ as a function of filler volume fraction of silica ${ }^{24 b}$.

Wolff has found that the ratio between the increase in rheometer torque during vulcanization of the filled compound and that of the gum is directly proportional to the filler loading $24,47,48$.

$$
\frac{D_{\max }-D_{\min }}{D_{\max }^{o}-D_{\min }^{o}}-1=\alpha_{f} \frac{m_{f}}{m_{p}}
$$

Where:

$D_{\max }-D_{\min } \quad$ is the maximum change in torque for filled rubber,

$D_{\text {max }}^{\circ}-D_{\text {min }} \quad$ is the maximum change in torque for gum rubber,

$m_{f} / m_{p} \quad$ is the weight ratio of filler to polymer,

$\alpha_{f} \quad$ is a filler specific constant which is independent of the curative system and closely related to the morphology of the filler.

In his investigation, Wolff defined the slope, $\alpha_{f}$ as the in-rubber structure. It is pointed out that $\alpha_{f}$ is a filler specific constant which is independent of the cure system and

\footnotetext{
${ }^{\mathrm{b}}$ Reproduced with permission from Rubber Chemistry and Technology. Copyright $@(1993)$, Rubber Division, American Chemical Society, Inc.
} 
closely related to the morphology of the filler. Here, $\alpha_{f}$ represents the structure of the filler as it exists in the vulcanizate after possible structure breakdown during mixing and vulcanization $^{49}$.

Figure 2.20 shows the plots of the normalized Wolff parameter, $\alpha_{f}$ against filler loading for both carbon black N110 and silica in NR and NBR ${ }^{46}$. It can be seen that the $\alpha_{f}$ of carbon black N110 is constant regardless of the filler loading both in NR and NBR. But for silica in NR the $\alpha_{f}$ is depending on the filler loading. In the case of NBR, a sharp upturn of $\alpha_{f}$ is observed at high silica loading but the increase in $\alpha_{f}$ is somewhat less than for NR.

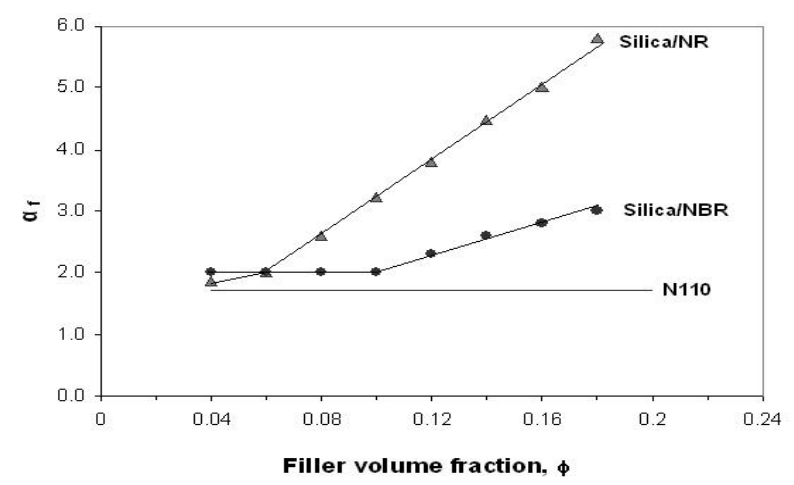

Figure 2.20 Wolff parameter $\alpha_{f}$ as a function of filler volume fraction for $N 110$ and silica in $N R$ and $N B R^{46 c}$.

The strong agglomeration of silica in the rubber matrix results in higher viscosity of the compounds, rapid increase in $\alpha_{f}$ and higher moduli of the vulcanizates at small strain: the latter commonly called the Payne effect ${ }^{50}$. The build-up of a strong filler network can be seen at high filler loading of silica, especially without silane modification ${ }^{51}$. The strong interaggregate interaction of silica as indicated by the Payne effect is illustrated in Figure $2.21^{32}$.

Mukhopadhyay has found silica to behave differently in comparison with carbon black in NR compounds. The $\alpha_{f}$ for carbon black is independent of curing temperature and dependent on filler characteristics only ${ }^{52}$. The larger the primary particle size of the carbon black, the lower the $\alpha_{f}$ value. Figure 2.22 illustrates the unusual behavior of silica where $\alpha_{f}$ value is dependent on the curing temperature.

\footnotetext{
${ }^{c}$ Reproduced with permission from Rubber Chemistry and Technology. Copyright @ (1993), Rubber Division, American Chemical Society, Inc.
} 


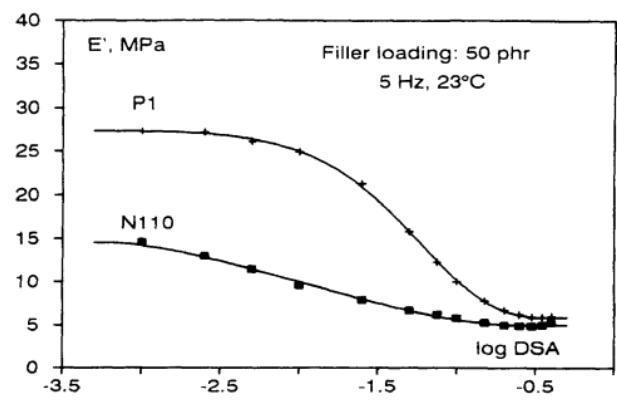

Figure 2.21 Comparison of Payne effect of NR vulcanisate at 50 phr loading between silica (P1) and carbon black (N110); E' as a function of the logarithm of the double strain amplitude ${ }^{32 d}$.

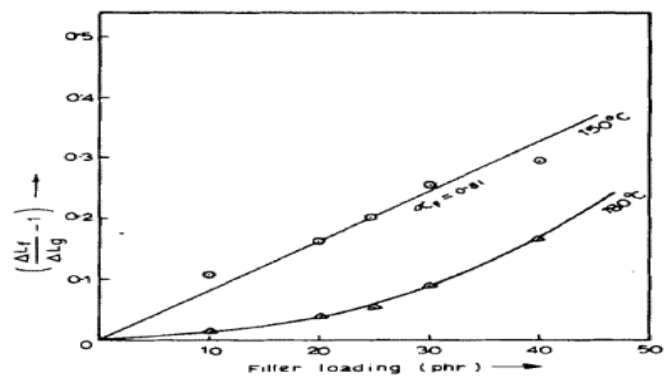

Figure 2.22 Plot of Wolff parameter, $\alpha_{f}\left(\Delta \mathrm{L}_{\mathrm{f}} / \Delta \mathrm{L}_{\mathrm{g}}-1\right)$ for silica-filled vulcanizates with vulcanization temperature indicated ${ }^{52 e}$.

The reinforcement mechanism of silica is different from carbon black. Kraus ${ }^{53}$ has shown that the swelling of a large number of vulcanizates containing highly reinforcing fillers obeys the following equation:

And

$$
\begin{aligned}
& v_{r o} / v_{r f}=1-m \phi /(1-\phi) \\
& m=3 C\left(1-v_{r o}{ }^{1 / 3}\right)+v_{r o}-1
\end{aligned}
$$

Where $v_{r o}$ is the volume fraction of rubber in unfilled (gum) vulcanizate,

$v_{r f}$ is the volume fraction of rubber in the filled vulcanizate,

$\phi$ is the volume fraction of filler in the filled vulcanizate,

$\mathrm{C}$ is a constant, characteristic of the filler but independent of the polymer, the solvent or the degree of vulcanization.

\footnotetext{
${ }^{d}$ Reproduced with permission from Rubber Chemistry and Technology. Copyright $@(1991)$, Rubber Division, American Chemical Society, Inc

${ }^{\text {e }}$ Reproduced with permission from Rubber Chemistry and Technology. Copyright $\odot$ (1979), Rubber Division, American Chemical Society, Inc
} 
The typical Kraus plot of $v_{r o} / v_{r f}$ versus $\phi /(1-\phi)$ for reinforcing and non-reinforcing fillers is shown in Figure $2.23^{54}$.

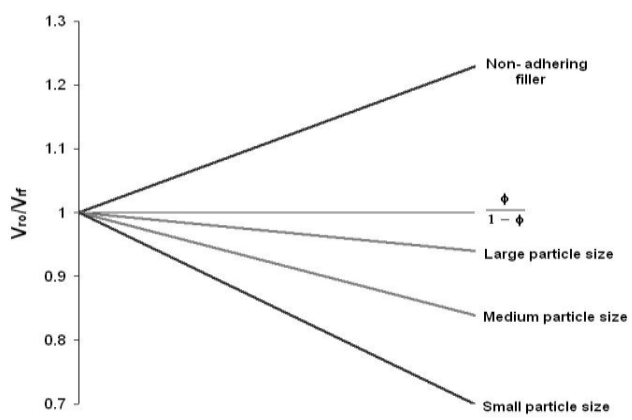

Figure 2.23 Relative swelling $R\left(=v_{r o} / v_{r f}\right)$ of filled vulcanizates as a function of filler loading ${ }^{54 t}$.

The extent of reinforcement can be determined from the Cunneen and Russell

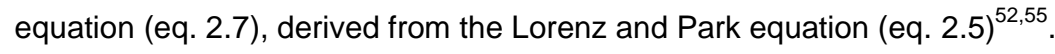

$$
Q_{f} / Q_{g}=a e^{-z}+b
$$

Also,

$$
\frac{Q_{f}}{Q_{g}}=\frac{v_{r o}\left(1-v_{r f}\right)}{v_{r f}\left(1-v_{r o}\right)} \cong \frac{v_{r o}}{v_{r f}}
$$

So,

$$
v_{r o} / v_{r f}=a e^{-z}+b
$$

Where: $z$ is the weight fraction of filler in the vulcanizates, $a$ and $b$ are constants characteristic of the system. By plotting $v_{r o} / v_{r f}$ against $e^{-z}$, values of a (slope) and $b$ (intercept) can be determined. The higher the value of $a$, the higher is the swelling restriction and reinforcement.

It is depicted in Figure $2.24^{52}$ that carbon blacks obey the Kraus equation up to a certain volume fraction of filler. Higher curing temperature causes a reduction in polymerfiller attachment as evident from the slope of the plots. Nonetheless, silica shows erratic behavior in the Kraus plot as compared to carbon black. Silica shows a positive slope indicative of poor interaction of the filler with the rubber. The most plausible explanation for the abnormal behavior of the silica-filled compounds is an ion exchange reaction on the silica surface between silanol groups and zinc stearate (Figure 2.25). In this reaction, stearic acid is liberated, which then solubilizes more zinc oxide, and the modified silica surface may be responsible for the deviations in the Kraus plots.

\footnotetext{
${ }^{\dagger}$ Reprinted from Polymer, 20, B.B. Boonstra, Role of Particulate filler in Elastomer Reinforcement: A Review, 691-704, Copyright (1979), with permission from Elsevier
} 


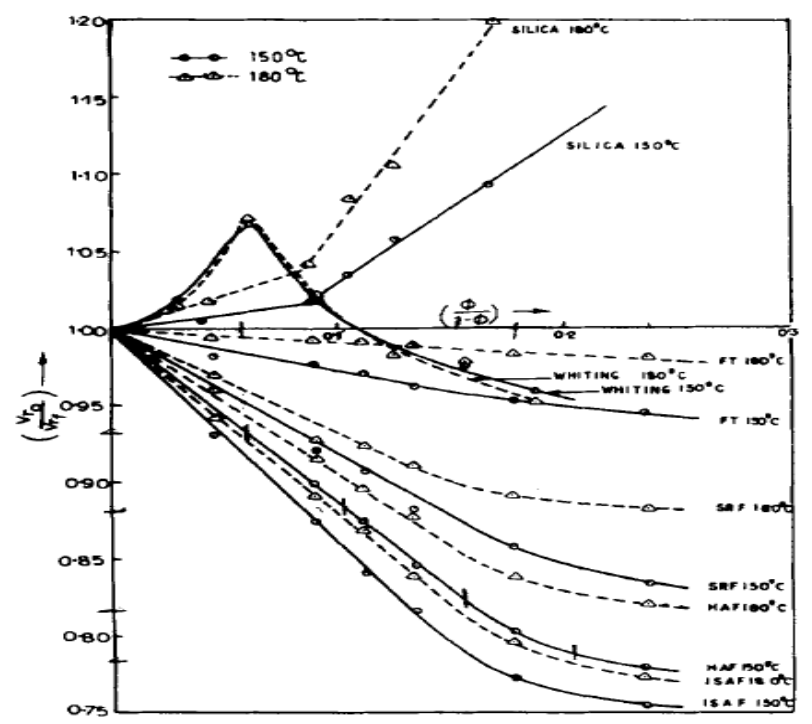

Figure 2.24 Kraus plots for different types of fillers: carbon black, silica \& whiting $^{52 g}$.
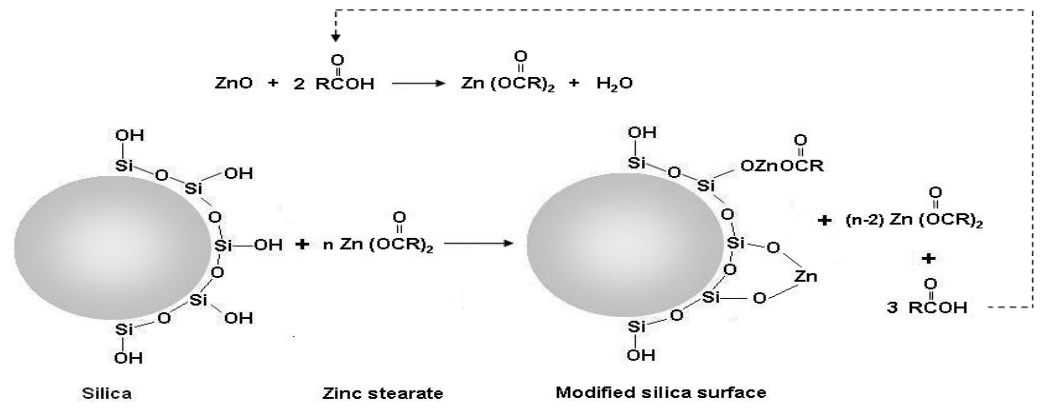

Figure 2.25 Reaction on the silica surface between silanol groups and zinc stearate.

Kralevich and Koenig have carried out FTIR analysis of silica-filled synthetic cis-14-polyisoprene, $I^{56}$. As shown in Figure 2.26, the increase in silica loading results in a decrease in wavenumber and rise of intensity in the silica region $\left(1250-1000 \mathrm{~cm}^{-1}\right)$. It is reasoned that changes in the FTIR spectrum are the result of the silica adsorbing the rubber and components of the cure system ingredients. The primary cause of the shifting and broadening is determined to be a combination of physisorption and chemisorption of the rubber matrix on the silica.

\footnotetext{
${ }^{9}$ Reproduced with permission from Rubber Chemistry and Technology. Copyright $\odot$ (1979), Rubber Division, American Chemical Society, Inc
} 


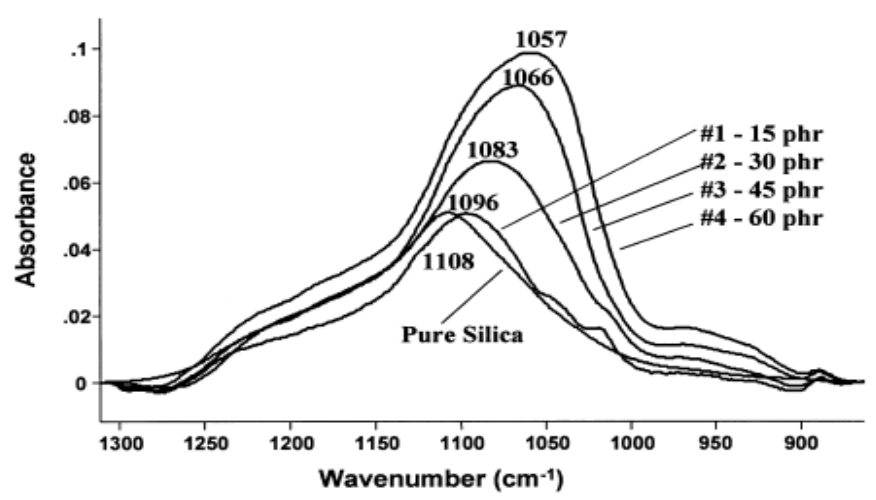

Figure 2.26 FTIR spectrum showing the effects of silica loading in rubber compound on Si-O-Si stretching ${ }^{56 h}$.

In a bound rubber study, Cook et al. has reported that a high level of NR is bound with silica, whilst in contrast very little BR is bound to silica without coupling agent (Table $2.8)^{57}$. It is postulated that hydrophilic proteins and occasional polar functional groups on NR are hydrogen bonded to silica. The volume swelling $(\mathrm{Vr})$ which is indicative of the strength of bound rubber shows higher values for NR than BR. Also noted is a high level of bound rubber for epoxidized natural rubber (ENR).

Table 2.8 Bound rubber content of uncured silica masterbatch of NR and $\mathrm{BR}^{57}$

\begin{tabular}{lccc}
\hline Property & BR & NR & ENR \\
\hline Bound rubber, g/g silica & 0.249 & 1.407 & 1.373 \\
Bound rubber content, \% & 5.0 & 28.2 & 27.5 \\
Volume swelling, Vr & 0.006 & 0.012 & 0.022 \\
\hline
\end{tabular}

The network visualization using TEM micrographs of a NR vulcanizate filled with 20 phr silica is illustrated in Figure $2.27^{57}$. It clearly shows voids between silica and the NR network. There are small numbers of stained NR network strands connecting silica particles with the surrounding rubber network. This provides direct evidence for interaction between NR and silica, which is in agreement with the results of bound rubber and volume swelling of silica-filled NR mentioned earlier.

\footnotetext{
${ }^{\mathrm{h}}$ Reproduced with permission from Rubber Chemistry and Technology. Copyright $\odot$ (1998), Rubber Division, American Chemical Society, Inc
} 


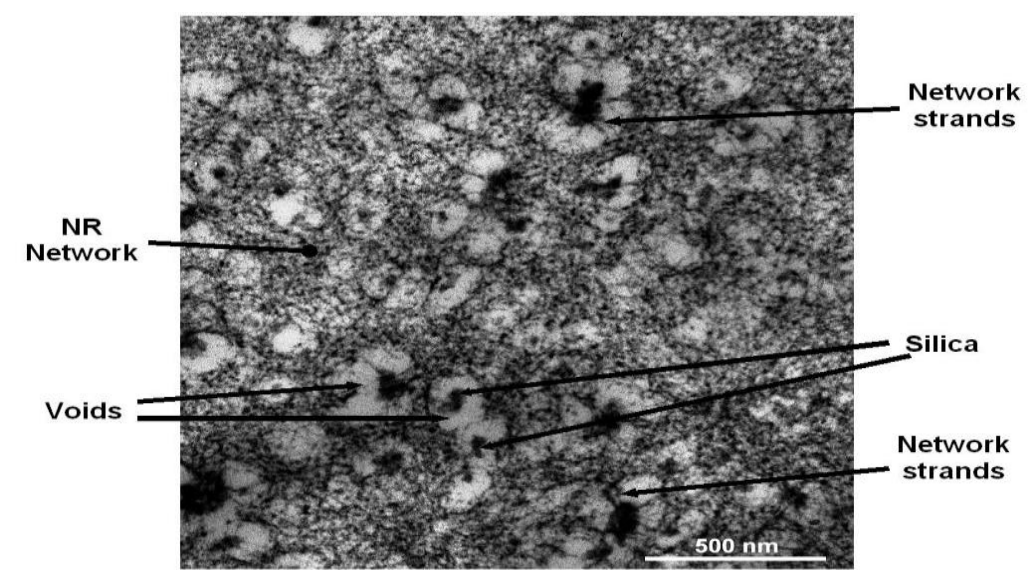

Figure 2.27 Network visualization TEM micrograph of NR vulcanizate filled with 20 phr silica ${ }^{57}$.

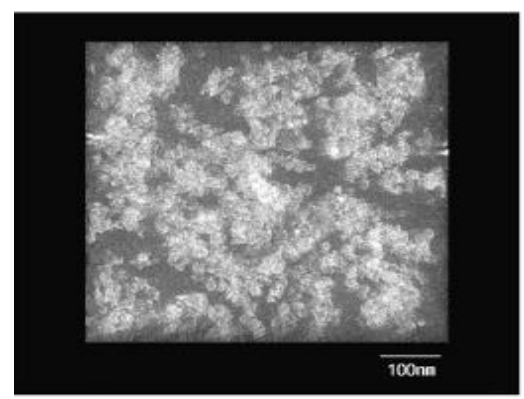

(a) NR-mix-V

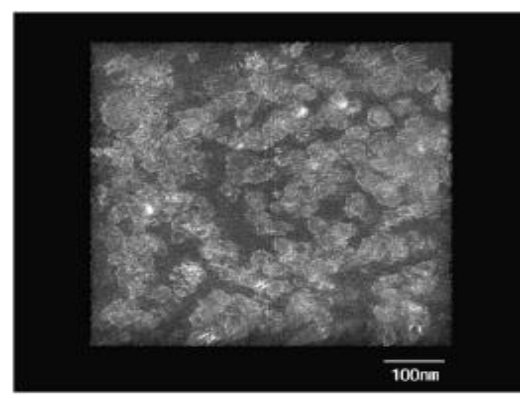

(b) NR-in situ-V

Figure 2.28 3D-TEM images of silica-filled NR vulcanizates ${ }^{58 i}$.

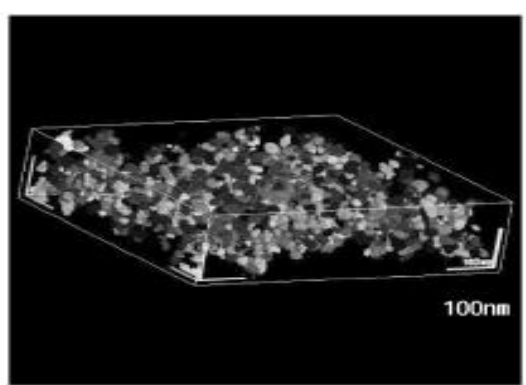

(a) NR-mix-V

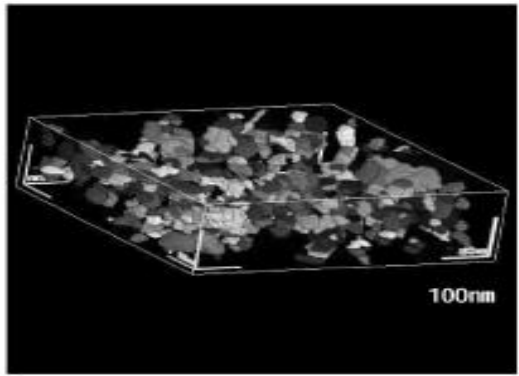

(b) NR-in situ-V

Figure 2.29 3D-image analysis results for silica-filled NR vulcanizates ${ }^{58 i}$.

\footnotetext{
${ }^{i}$ Reproduced with permission from Rubber Chemistry and Technology. Copyright $@$ (2007), Rubber Division, American Chemical Society, Inc
} 
Kato and coworker have successfully visualized the three-dimensional (3D) morphology of silica aggregates in sulfur-cured NR vulcanizates at nanometer level by applying transmission electron microscopy (TEM) combined with computerized tomography, so-called $3 \mathrm{D}-\mathrm{TEM}^{58}$. As pictured in Figure 2.28, the white particles indicate silica aggregates. NR-mix-V is sulphur-cured NR with 33 phr of silica Ultrasil VN3, whilst NR-in situ-V is 33 phr silica generated in raw rubber by a sol-gel reaction. As shown in Figure 2.29 the silica aggregates can be distinguished by the colours. The three white lines in the four corners of the images are 3D scales, the length of the scale indicates 100 nanometers. The primary silica particles in the NR-in situ vulcanizate are larger compared with the NR-mix vulcanizate.

\subsubsection{The Use of Silica With Coupling Agent}

When organosilane coupling was first introduced in 1975, the work by F.Thurn et al. ${ }^{59}$ has shown that bis-(3-triethoxysilylpropyl)-trisulfide or bis-(3-triethoxysilylpropyl)-tetrasulfide (TESPT) have an effect as reinforcing additive for silica-filled natural rubber compounds. $A$ compound of natural rubber filled with $40 \mathrm{phr}$ of silica and using polysulfide organosilane as coupling agent gives vulcanizate properties superior to a silane-free vulcanizate, especially in tensile strength and modulus at $300 \%$. The use of polysulfide organosilane did shorten the scorch time but did not negatively affect the Mooney viscosity. However, the use of 3mercaptopropyl-trimethoxysilane in the natural rubber-silica compound caused premature scorching and limited further processing possibilities. The effect of TESPT on the network of silica compounded with natural rubber was investigated further by Wolff ${ }^{60}$.

Silanization of silica leads to fundamental changes in its reinforcing characteristic. The dispersive component of surface energy of modified-silica drops below the value of unmodified silicas and the polar component becomes negligible. The measurement of the Payne effect also shows that TESPT-modified silica falls below that of carbon black as depicted in Figure $2.30^{49}$. In addition, Wolff reported that the viscosity of a silica compound can be reduced to a carbon black-level or even lower with silane TESPT modification (Figure 2.31) ${ }^{45}$.

Evaluation of the in-rubber structure of modified silica by Wolff has shown that silane modification gives silica $\alpha_{f}$ values corresponding to those of reinforcing carbon black (Table 2.9 $)^{45}$. The silane modification lowered the structure and increased the surface. The hydrophobation of silica with silane dissolves the bonds responsible for high secondary agglomeration of silica (primarily hydrogen bonds and perhaps -Si-O-Si- bonds). The build up of filler-to-rubber bonds is the reason for the increase of the in-rubber surface area. 

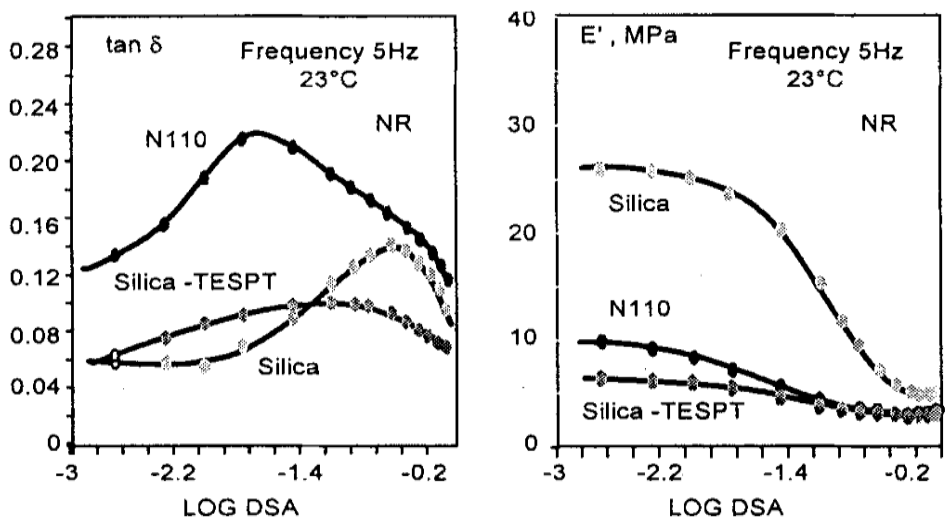

Figure 2.30 Tan delta and E' as functions of the double strain amplitude (DSA) for carbon black N110, silica and TESPT-modified silica compounds ${ }^{49 j}$
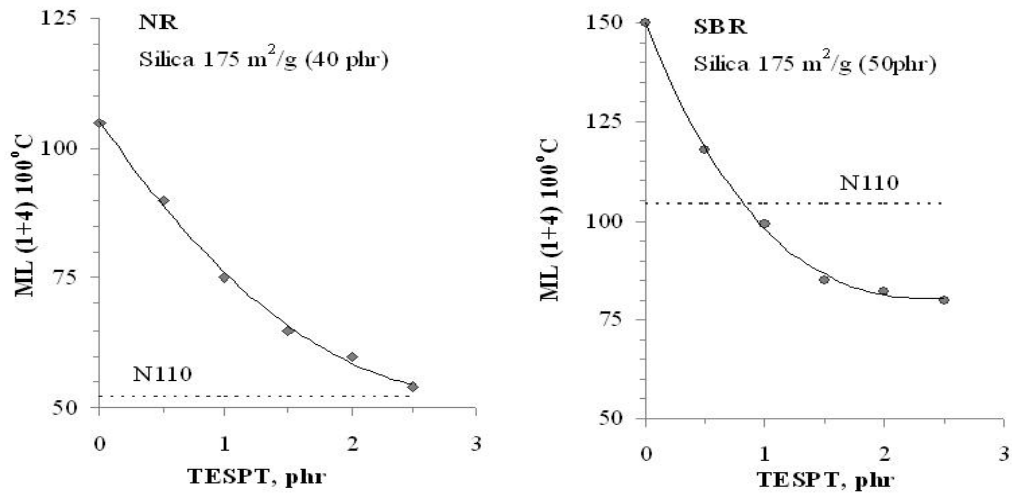

Figure 2.31 Decrease in Mooney viscosity with increasing TESPT used ${ }^{49}$

Table 2.9 In-rubber structure of carbon black and silica ${ }^{45}$

\begin{tabular}{lc}
\hline Filler & $\alpha_{f}$ values \\
\hline Carbon Black Corax N110 & 1.86 \\
Silica Ultrasil VN2 & 5.65 \\
Silica Ultrasil VN2-silane modified & 1.84 \\
\hline
\end{tabular}

\footnotetext{
${ }^{\mathrm{j}}$ Reproduced with permission from Rubber Chemistry and Technology. Copyright $@$ (1996), Rubber
} Division, American Chemical Society, Inc 
Görl et al. have shown that pre-crosslinking or scorching at higher temperatures concerns a partial formation of rubber to filler bonds as illustrated in the rheological behavior of NR/TESPT and NR/TESPT/silica compounds (Figure 2.32$)^{61}$. A notable torque rise only takes place when filler is present in the compound, especially at $180^{\circ} \mathrm{C}$.
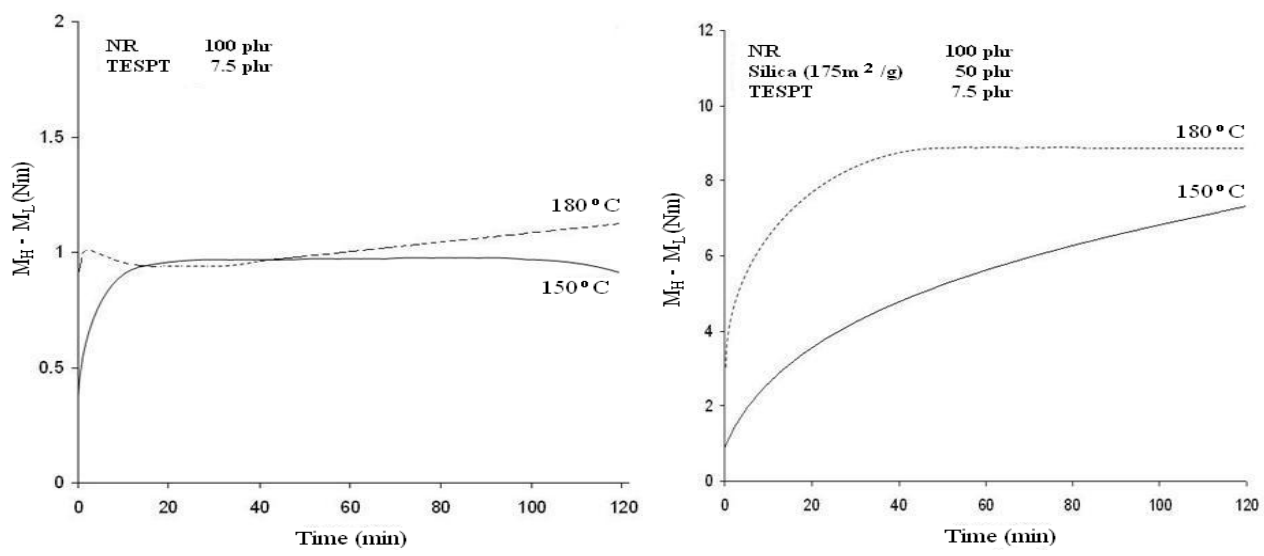

Figure 2.32 Rheological behavior of NR/TESPT and NR/TESPT/silica compounds at 150 and $180^{\circ} \mathrm{C}$, respectively ${ }^{61}$.

Ansarifar and coworker ${ }^{62}$ have examined the effect of treating the silica with TESPT in sulfur-cured NR. The bound rubber of a NR compound containing 30 phr of silica increased with increment of TESPT loading. The tensile strength, elongation at break, stored energy density and cohesive tear strength of the rubber were improved significantly after the full amount of TESPT was introduced into the compound ( $6 \mathrm{phr}$ ). Nonetheless, the in situ modification reaction of silica with TESPT was inefficient with the mixing condition used and the filler was poorly dispersed in the rubber. Silica which had not been properly silanized would result in deficient dispersion in rubber, and might have adverse effects to reinforce rubber ${ }^{63}$.

A study on TESPT pre-treated precipitated silica has shown that it is effective as filler to crosslink and reinforce $\mathrm{NR}^{64}$. An investigation by Ansarifar on the effect of $60 \mathrm{phr}$ TESPT pre-treated silica (Degussa Coupsil 8113) on the mechanical properties of sulfurcured NR showed that the tensile strength, stored energy density at break, hardness and tearing energy increased, but strain at break, cyclic fatigue life and compression set deteriorated $^{65}$. When the silica-filled NR was cured primarily by using sulfur in TESPT, the properties of the vulcanizate were enhanced. The addition of $0.2 \mathrm{phr}$ sulfur to the cure 
system had a noticeable improvement effect on the properties of filled NR, especially in tearing energy and fatigue life.

$\mathrm{Pal}$ and coworker ${ }^{55}$ investigated the effect of reinforcing silica on vulcanization, network structure and technical properties of natural rubber. Reinforcement of silica in a NR mix with coupling agent TESPT was evident at higher filler loading (40 phr). It was reported that the network structure did not change significantly on addition of silica and TESPT, particularly at optimum cure times.

Bokobza and Olivier have combined different experimental techniques (infrared dichroism, birefringence, mechanical properties) to study the reinforcement mechanism of filled natural rubber based on measurements of chain orientation ${ }^{66}$. The Payne and Mullins effects were also evaluated (Figure 2.33). It was demonstrated at intermediate strains, that the increase in moduli can be explained by the inclusion of rigid particles in the soft matrix and from molecular interactions between rubber and filler. The reinforcement of silica in NR with the use of silane coupling agent could also be obtained in NR composites containing silica particles generated by the sol-gel process ${ }^{67}$.
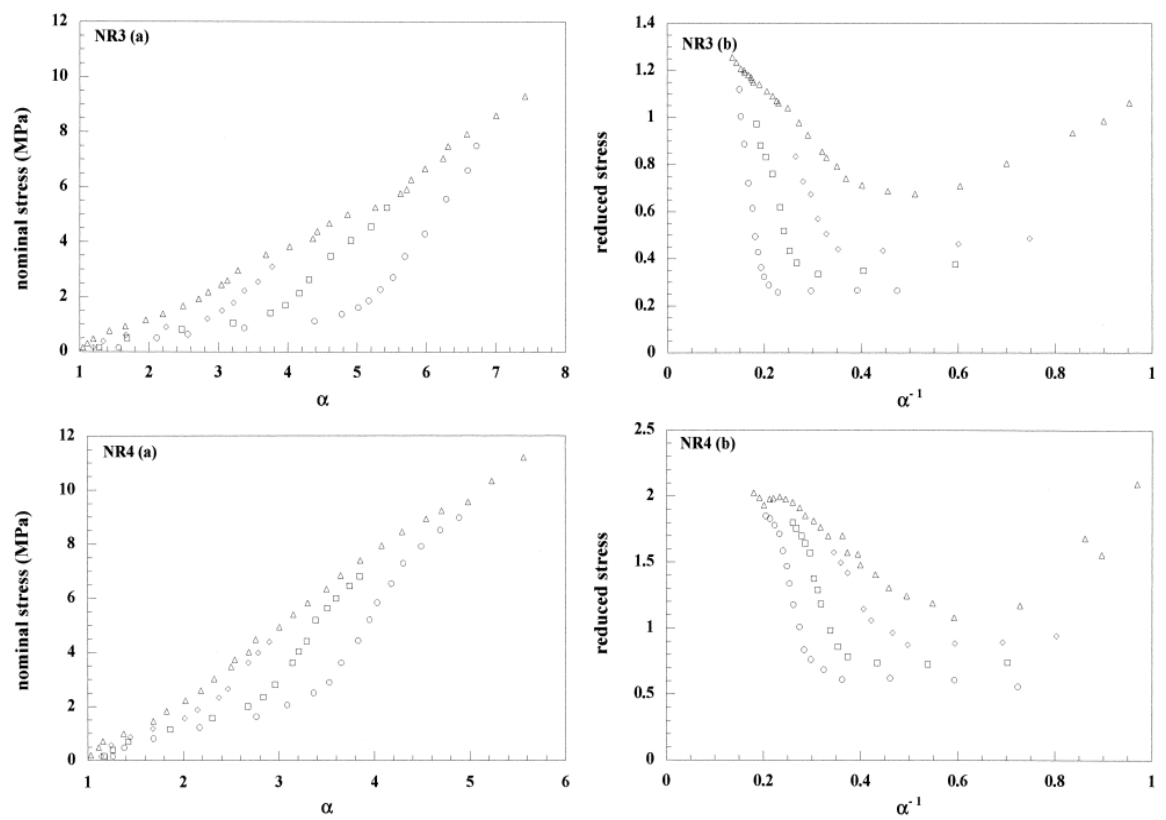

Figure 2.33 Mullins hysteresis from first and second stretching curves (a) and the corresponding Mooney-Rivlin plots (b) for silica-filled NR (NR3) and carbon black-filled NR $(N R 4)^{66}$. 
Chung and Dae have investigated the effect of different rubber types in the rubber system based on NR with silica reinforcement ${ }^{68}$. A reasonable rubber system comprising NR and SBR with high styrene content was considered the most appropriate for application in tire tread materials.

\subsection{Mixing Silica INTO RUbBer CompoundS}

The mixing of silica filled rubber compounds with in-situ modification of silica with a coupling agent in an internal mixer is a challenging task. During the mixing of silica compounds, two factors need to be taken into account, which are the mixing sequence of specific ingredients and the completion of the modification reaction of silica with TESPT ${ }^{42,69}$. For this, all compounding ingredients which might interfere with the ethoxy groups of TESPT have to be excluded during the modification reaction of silica with TESPT. The reaction of TESPT with silica during mixing or in situ is always more economical than using modified silica. Hence, a prerequisite for this is the modification reaction in the compound to be completed during the mixing.
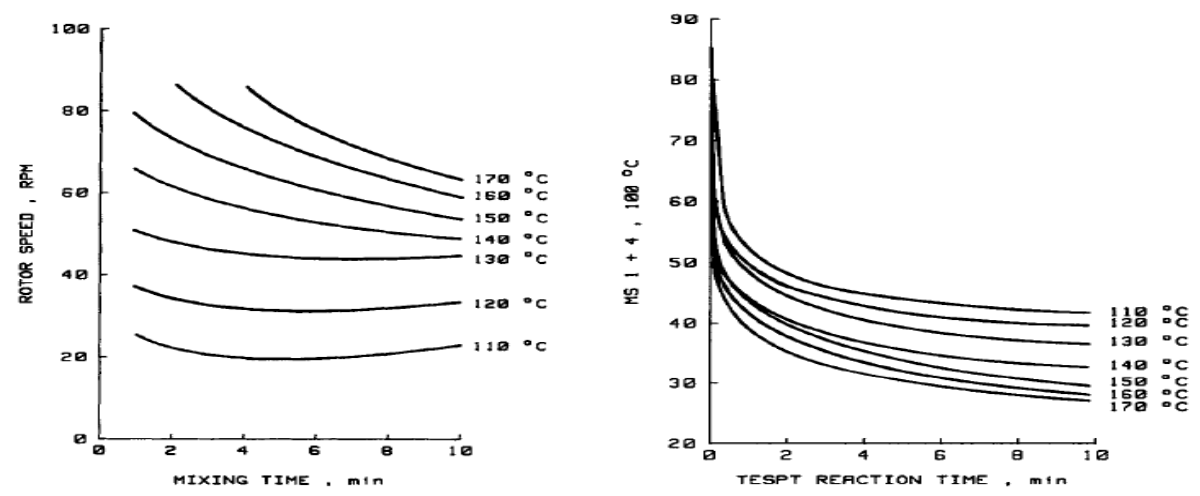

Figure 2.34 (a): Rotor speed as a function of mixing time to achieve batch temperatures from 110 to $170^{\circ} C^{69 k}$; (b): Mooney viscosity of a silica-NR compound as a function of temperature and mixing time ${ }^{69 k}$.

Extensive studies have been carried out by Wolff ${ }^{69}$ and Reuvekamp ${ }^{41}$ on the variation of mixing temperature and time during the modification of silica with TESPT. Figure 2.34(a) illustrates the correlation of reaction times and rotor speed to produce the desired batch end temperature between 110 and $170^{\circ} \mathrm{C}$. This is based on a typical off-the-road tire

\footnotetext{
${ }^{\mathrm{k}}$ Reproduced with permission from Rubber Chemistry and Technology. Copyright $\odot$ (1982), Rubber Division, American Chemical Society, Inc
} 
tread (OTR) formulation using 100 phr NR, 20 phr silica, 3 phr TESPT and 40 phr carbon black, carried out in a 2.5 liter laboratory internal mixer. The effect of reaction time and temperature on viscosity of the compound is depicted in Figure 2.34(b). The longest reaction times produce the lowest compound viscosity and the highest end temperatures produce the largest drop in viscosity with reaction time.

Wolff has concluded that the degree of modification increases with TESPT reaction time and temperature, where the effect of temperature is more significant than time. To obtain the highest filler-rubber to rubber-rubber bond ratio, the mix should be at highest possible temperature within practical limits. This is more important than increasing the mixing time. This study also showed that the modification reaction of silica with TESPT in situ cannot be considered as an equilibrium reaction.

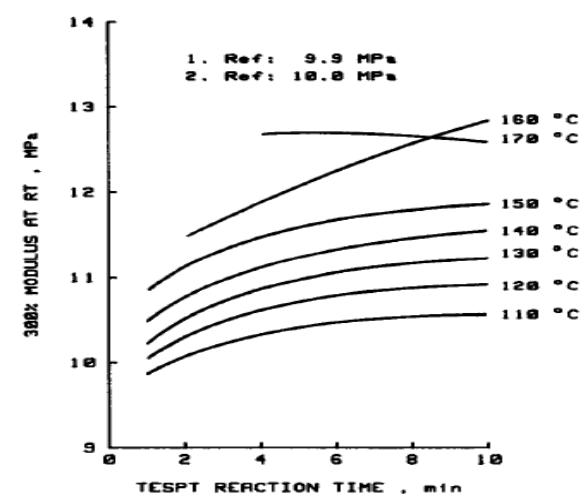

(a)

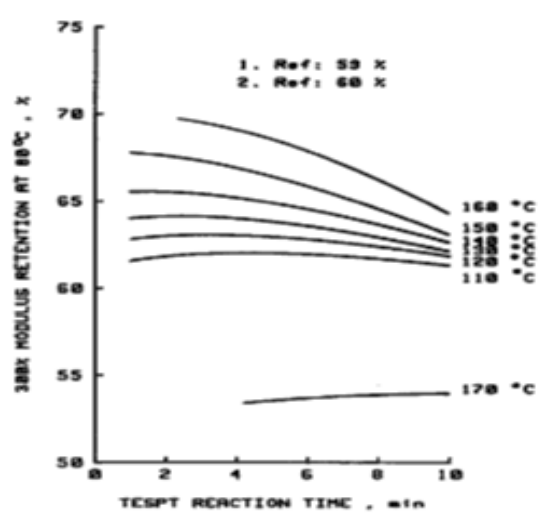

(b)

Figure 2.35 Modulus at 300\% (a) and retention at $80^{\circ} \mathrm{C}$ in $300 \%$ modulus (b) as a function of TESPT modification temperature and time ${ }^{691}$.

According to Wolff, for natural rubber, the highest and most practical modification reaction temperature is about $160^{\circ} C^{69}$. The influence of TESPT reaction time and temperature on $300 \%$ modulus is depicted in Figure 2.35. At a temperature between $150^{\circ} \mathrm{C}$ and $160^{\circ} \mathrm{C}$, the reaction produces optimum cure rate and $300 \%$ modulus. However, above $160^{\circ} \mathrm{C}$, the thermal reaction of TESPT with natural rubber starts and reduces the sum of filler/rubber and rubber/rubber crosslinks available in the vulcanizate. Up to $160^{\circ} \mathrm{C}$ the number of filler/rubber and rubber/rubber crosslinks remains constant. By increasing the

\footnotetext{
' Reproduced with permission from Rubber Chemistry and Technology. Copyright @ (1982), Rubber Division, American Chemical Society, Inc.
} 
temperature higher than $160^{\circ} \mathrm{C}$ and reaction time lead to an increasing ratio of filler/rubber to rubber/rubber bonds.

In order to achieve the highest silica modification with TESPT, the TESPT should be added together with silica in the mixing sequence, to make the best of the reaction time dependency. It is also observed that by using optimum reaction temperature and time, less TESPT is needed to obtain higher filler/rubber bonding and desired properties.

Sandstrom et al. have reported on the effect of the order of addition of silica and carbon black into the rubber compound during mixing ${ }^{38}$. The silica reinforced tread compound utilised 100 phr NR, 30 phr silica, 6 phr coupling agent and 50 phr of carbon black. They recommend the addition of carbon black in the first non-productive stage and followed by silica addition in the second non-productive stage. The compound prepared with silica added in the second stage gave superior processing behavior and produced less fragments of compound when dumped from the internal mixer and subsequently had better milling behavior.

Luginsland $^{70}$ in his work has summarized that to complete the silanization reaction and to guarantee the release of ethanol, mixing time must be sufficiently long. The mixing temperature should be high enough to speed up the reaction with the silica, but excessive temperatures, which lead to unwanted pre-crosslinking, must be avoided. The silica should have enough moisture content, about $5 \%{ }^{71}$ to complete the silanization reaction to an acceptable level. In the case of TESPT, a mixing temperature in the range of $145-155^{\circ} \mathrm{C}$ is advisable, to achieve a good silanization and to avoid the thermal pre-crosslinking. This requires a mixing equipment with a precise temperature control.

The study by Reuvekamp on silica-filled S-SBR/BR compounds showed that dump temperature is the dominant parameter in mixing silica and rubber with the aid of TESPT as a coupling agent ${ }^{41}$. A minimum dump temperature of $130^{\circ} \mathrm{C}$ is required to ensure that the silica and the coupling agent react to the desired extent, but at temperatures above $160^{\circ} \mathrm{C}$ either the coupling agent starts to react with the rubber or the TESPT starts to donate sulfur. $\mathrm{He}$ also found that a mixing of at least 10 minutes at $150^{\circ} \mathrm{C}$ is necessary to ensure complete coupling of the silica and the silane and the reaction between the silica and the silane takes place primarily during the first mixing $\operatorname{step}^{72}$. As illustrated in Figure 2.36 the storage modulus at $0.56 \%$ strain did not reach a constant value after the first mixing step. However, in the second mixing cycle of 5 minutes, the G' value reached a constant value for the compound that had been mixed for more than 10 minutes in the first step.

Reuvekamp also revealed that the presence of zinc oxide in the mixer provokes premature scorch of the compound, due to its interference with the reaction between the 
coupling agent and the silica surface. A lower tendency to scorch was observed when zinc oxide was omitted during the internal mixing stage and added only later together with the curing additives on the two-roll mill.
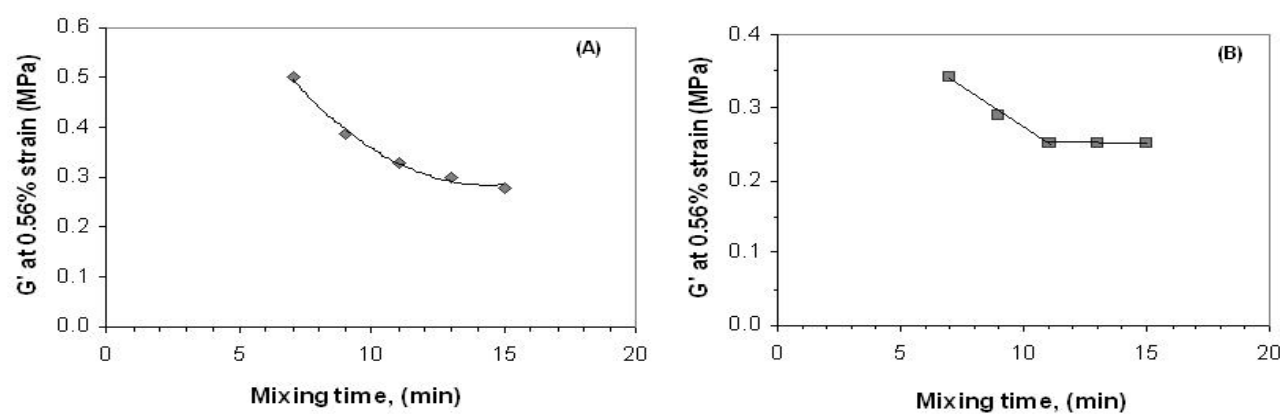

Figure 2.36 The influence of mixing time on the Payne effect, storage modulus at $0.56 \%$ strain after the first mixing step $(A)$ and after the second mixing step $(B)^{41}$.

In order to achieve a sufficient degree of silanization during the mixing cycle, a temperature above $130^{\circ} \mathrm{C}$ is required. The upper limit of the temperature window during processing is given by the risk of scorch. Therefore, the temperature range for the silanization reaction is limited, from approximately $130^{\circ} \mathrm{C}$ to $150^{\circ} \mathrm{C}$, depending on the type of coupling agent and silica, the mixing process and the type of mixing equipment ${ }^{42}$. Hence silica compounds need several mixing stages in order to stay in this relatively narrow temperature window and close to $150^{\circ} \mathrm{C}$.

Dierkes has investigated the influence of different processing parameters of the mixing on the silanization efficiency ${ }^{42}$. Silanization in an open mixer, where ethanol can escape out of the mixing chamber results in a lower Payne effect. The fill factor also influences the silanization efficiency. The lower the fill factor, the lower the total amount of ethanol generated in the mixing chamber. This is due to the fact that the batch is sheeted out to a higher extent and evaporation of ethanol out of the compound is enhanced. The concentration of ethanol in the compound and in the vapor phase of the mixing chamber will affect the rate of silanization as ethanol is competing with the silane for absorption sites on the silica surface. The influence of the fill factor on the Payne effect is more pronounced in an intermeshing mixer than in a tangential mixer due to the more irregular intake behavior. $\mathrm{A}$ summary of the different processing parameters which are identified to have an influence on the silanization efficiency is depicted in Table 2.10. 
Table 2.10 The influence of different processing parameters on silanization efficiency ${ }^{42}$

\begin{tabular}{|c|c|c|c|c|c|c|c|c|}
\hline $\begin{array}{r}\text { Factors } \\
\text { improving } \\
\text { the silanization } \\
\text { efficiency }\end{array}$ & 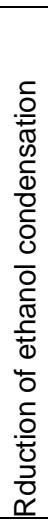 & 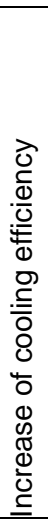 & 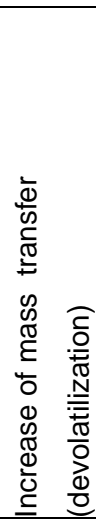 & 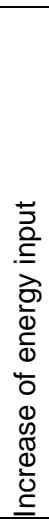 & 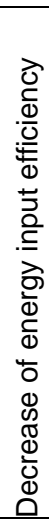 & 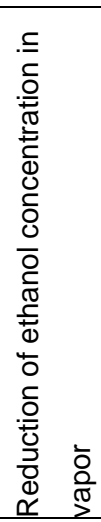 & 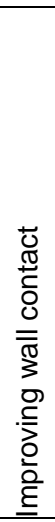 & 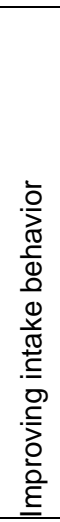 \\
\hline Rotor speed & & $\uparrow$ & $\uparrow$ & & & & & $\mathrm{x}$ \\
\hline Fill factor & $\mathrm{x}$ & $\bar{\uparrow}$ & $\downarrow$ & & $\downarrow$ & $\downarrow$ & $\uparrow$ & $\mathrm{x}$ \\
\hline Compound temperature & & $\uparrow$ & $\uparrow$ & $\uparrow$ & $\uparrow$ & $\downarrow$ & & $\mathrm{x}$ \\
\hline Open/closed mixer & 0 & c & 0 & & $x$ & 0 & c & c \\
\hline Air injection & $\uparrow$ & $\uparrow$ & $\uparrow$ & $\uparrow$ & & $\uparrow$ & & \\
\hline Coupling agent & $\mathrm{x}$ & & & & $\mathrm{x}$ & $\mathrm{x}$ & & \\
\hline
\end{tabular}

$\uparrow$ increase, $\downarrow$ decrease of the processing parameter for improvement, $x$ No clear influence

\subsection{Properties of Silica Reinforced Rubber Compounds in Relation to}

\section{TIRE PROPERTIES}

The first use of silica (5-10 phr) in truck tire treads based on NR had shown an improvement in tear properties in terms of cut and chip behavior, but the amount used was limited to avoid reduction in tread wear. Higher amounts of silica require coupling agents.

In 1992, Michelin patented the silica-filled rubber compound for the production of high-performance tires in all seasons ${ }^{73}$, which provide an excellent compromise of low rolling resistance, good traction on wet and snow-covered grounds, as well as noise reduction, later known as "Green Tire" technology. The rubber compound utilized a blend of high-vinyl solution-SBR and high cis-BR filled with highly dispersible silica ${ }^{74}$ (BET $190 \mathrm{~m}^{2} / \mathrm{g}$, CTAB $160 \mathrm{~m}^{2} / \mathrm{g}$ ) at high loading of $80 \mathrm{phr}$ and organosilane coupling agent (Degussa X50S) which is a mixture in a ratio of $1: 1$ of carbon black N330 and TESPT. It is highlighted in the patent that the compromise of magic triangle properties for the Michelin 'Green Tire' compound based on S-SBR and highly dispersible silica is superior to a compound using emulsion SBR and conventional silica. Nonetheless, the compound may also use a blend of S-SBR with another copolymer such as natural rubber or polyisoprene or butadiene rubber for up to 70 phr. 
Investigations by Wolff in field tests with truck treads based on NR demonstrated that the rolling resistance can be improved by as much as $30 \%$ when TESPT-modified silica is used to replace $\mathrm{N} 220$ carbon black. The treadwear index (abrasion resistance) decreases by no more than $5 \%$ and wet traction shows little changes ${ }^{49}$ (Figure 2.37 ).

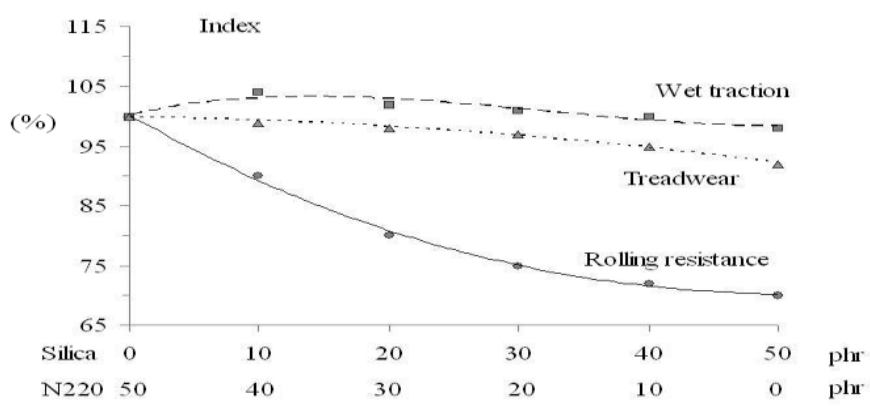

Figure 2.37 Tread test results TESPT- modified silica vs. N220 carbon black loading in NR truck treads ${ }^{49 m}$.

Silica has a superior influence on rolling resistance (tan $\delta$ at $60^{\circ} \mathrm{C}$ ) and lower heat buildup $\left(\Delta \mathrm{T}_{\text {centre }}\right)$ as compared to carbon black in NR compounds as shown in Figure $2.38^{75}$. Even silica at lower BET surface area offers a lower tan $\delta$ value and less heat build-up, in combination with good reinforcing behavior than a low reinforcing carbon black ${ }^{26}$.

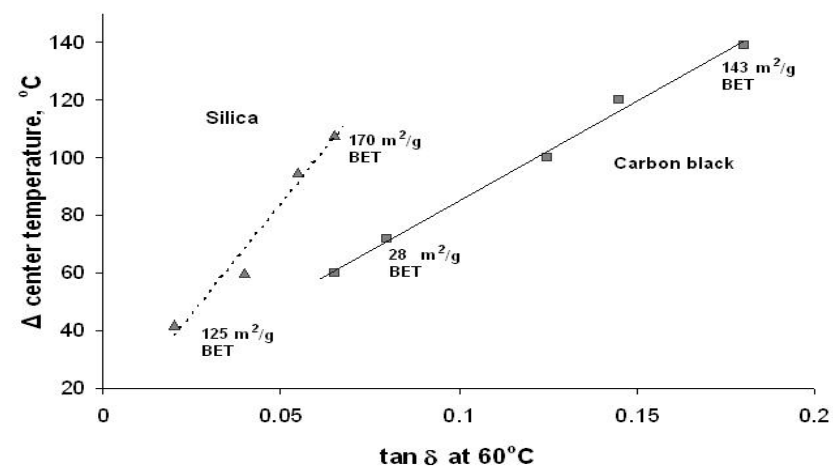

Figure 2.38 Influence of silica and carbon black on $\tan \delta$ at $60^{\circ} \mathrm{C}$ and heat build-up in NR, 50 phr filler ${ }^{75}$.

\footnotetext{
${ }^{\mathrm{m}}$ Reproduced with permission from Rubber Chemistry and Technology. Copyright @ (1996), Rubber Division, American Chemical Society, Inc
} 
As reported by Wolff, the rubber vulcanization reaction with TESPT has an effect on vulcanizate data resulting in two dominant types of vulcanizates. One is no reversion with low compression set and good ageing properties but low tensile and tear properties. The other one is no reversion with high tensile and tear properties, but high compression set. The effect of TESPT on physical data of silica vulcanizates is summarized in Table 2.11.

Table 2.11 Achievable physical properties with TESPT cure system ${ }^{45}$

\begin{tabular}{ccl}
\hline Accelerator & $\begin{array}{c}\text { Main structure of } \\
\text { crosslinks }\end{array}$ & \multicolumn{1}{c}{ Vulcanizate Properties } \\
\hline Thiuram-disulfides & C-S-C (monosulfidic) & No Reversion \\
& C-S ${ }_{2}-\mathrm{C}$ (disulfidic) & $\begin{array}{l}\text { Good ageing resistance } \\
\text { Low compression set }\end{array}$ \\
& & Extremely low heat build up \\
Sulfenamides & C-S ${ }_{x}-\mathrm{C}$ (polysulfidic) & No Reversion \\
and disulfides & C-S ${ }_{2}-\mathrm{C}$ (disulfidic) & High tensile and tear strength \\
& & High compression set \\
& Equilibrium cure (EC) & Extremely low heat build up \\
& system & \\
\hline
\end{tabular}

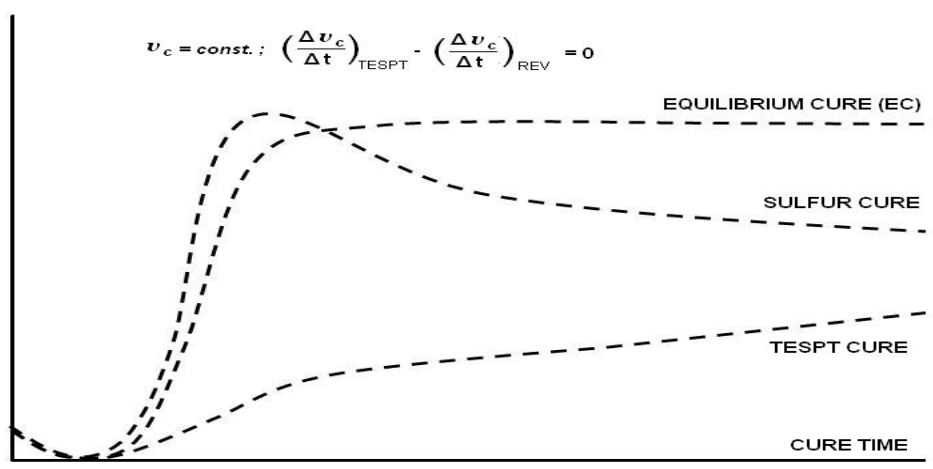

Figure 2.39 Equilibrium cure system for Off the Road (OTR) tire ${ }^{45}$.

The use of silica and TESPT was also applied in an Earth Mover tire tread recipe ${ }^{59}$. Based on 100 phr natural rubber and 66 phr of a mixture of silica Ultrasil VN3 and TESPT in 10:1 ratio, the properties were compared with a conventional carbon black reinforced EarthMover tire compound. It was illustrated that the properties of silanized-silica reinforced 
rubber exceed those of carbon black reinforced rubber especially in regard to tear resistance and heat build-up (Goodrich Flexometer test). The scorch time and cure time are somewhat shortened but still remain in a range suitable for industrial practice. The reduction in Mooney viscosity of the compound is also an added advantage for easier processing and lower production cost.

Wolff has reported that the use of silica in an Earth Mover (EM)- tire tread gives good properties ${ }^{45}$. The EM-tread compound was based on NR with 40 phr of N110, 20 phr of silica and 3 phr of TESPT. Here, Wolff introduced an equilibrium cure (EC) system ${ }^{n}$ consisting of TESPT and accelerator in a particular ratio dependent on test conditions. This system could be subjected to longer cure periods. The advantage of the EC cure system was the reversion and ageing resistance especially with regard to the NR compound: Figure 2.39. As shown in Table 2.12, the modification of silica with TESPT (EM2) leads to filler-to rubber bonds which increases the roadwear index and gives low heat build-up.

Table 2.12 EM-tread compound based on $\mathrm{NR}^{45}$

\begin{tabular}{lll}
\hline & EM1 & EM2 \\
\hline Tensile strength (MPa) & 18.6 & 21.3 \\
Trouser tear (N/mm) & 20 & 22 \\
Road wear index (\%) & 100 & 115 \\
Heat build-up ${ }^{\mathrm{a}}\left({ }^{\circ} \mathrm{C}\right)$ & 103 & 82 \\
\hline Ingredients in phr: EM1 (NR-100, N220-42, VN3-12, MBS-1.2, TMTM-0.1, retarder-0.3, $^{\text {sulfur-2) ; EM2 (NR-100, N220-40, VN3-20, TESPT-3, MBS-1.4, sulfur-1.7). }}$ \\
\hline \multicolumn{2}{c}{${ }^{\mathrm{a} G o o d r i c h ~ F l e x o m e t e r ~ 0.250 " / R T / 108 N / 60 ' ~}$}
\end{tabular}

The efficiency of the EC-system is particularly useful for application in truck tires which are exposed to considerable temperature during vulcanization. Even semi-efficientcured compounds suffer under reversion for carbon black-filled treads. The use of an ECsystem stops the reversion completely at any cure temperature ${ }^{76}$.

The use of a silica-TESPT system in heavy-duty solid tires has increased their life time resulting from the thermal resistivity of the compound. When silica-filled natural rubber compounds vulcanized with an EC-system were compared with an in-service compound carbon black-filled SBR sulfur cured, the heat generation of the silica-filled NR compound was remarkably lower: Table $2.13^{45}$. The blow-out testing on the Goodrich Flexometer could

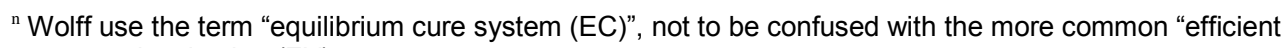
system vulcanization $(E V)$.
} 
not be finished due to the extremely low heat build-up of the silica-NR compound which did not lead to a continuous increase in temperature for thermal destruction of the vulcanizate.

Table 2.13 The physical data of silica-NR heavy duty solid tire in comparison with carbon black $^{45}$

\begin{tabular}{lcc}
\hline & SBR/CB & NR/Silica \\
\hline Tensile strength (MPa) & 19.3 & 24.8 \\
Modulus at 300\% (MPa) & 10.8 & 10.7 \\
Elongation (\%) & 460 & 510 \\
Hardness (Shore) & 61 & 61 \\
Goodrich Flexometer (0.25"/108N/RT): & & \\
$\Delta$ T after 120' $\left({ }^{\circ} \mathrm{C} /{ }^{\circ} \mathrm{F}\right)$ & $124 / 223$ & $30 / 54$ \\
Blow-out (min) & 139 & $>>4320$ \\
\hline
\end{tabular}

Reuvekamp has investigated the effect of silica/coupling agent combinations on the properties of NR compounds ${ }^{41}$. Addition of highly dispersible silica to NR reinforces this rubber. Comparing silica to carbon black, silica shows stronger reinforcing capabilities and better dynamic properties (low stiffness ratio) in NR compounds: Figure 2.40.
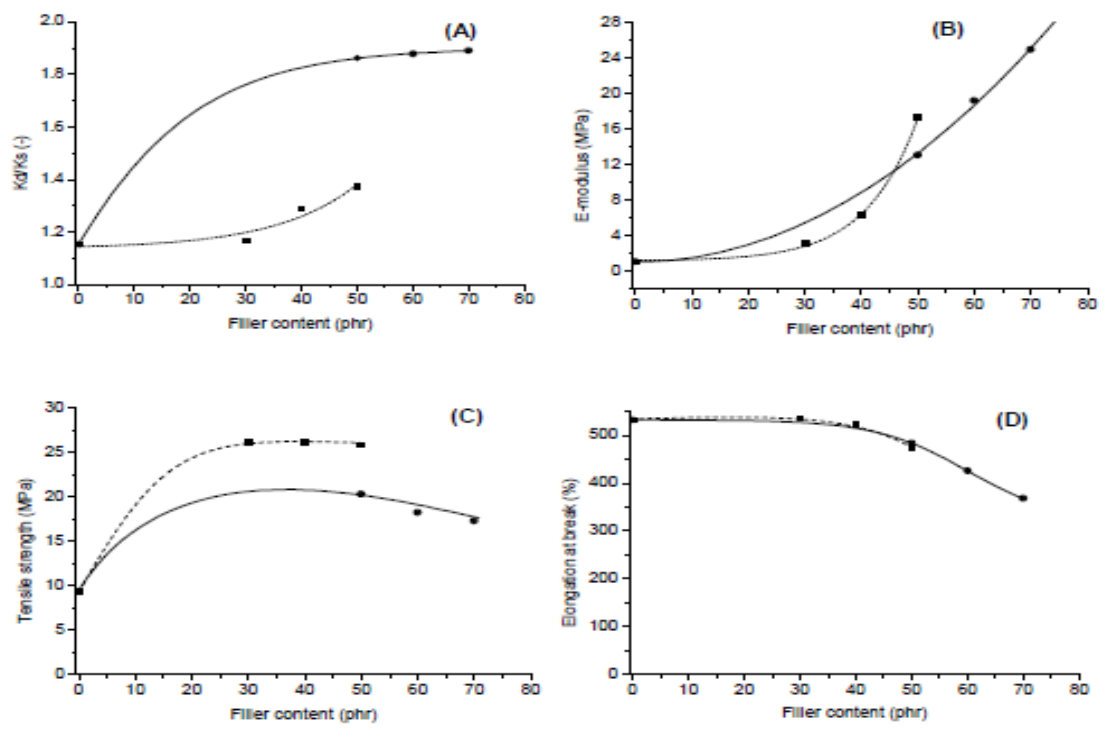

Figure 2.40: Dynamic and mechanical properties as a function of the filler content.

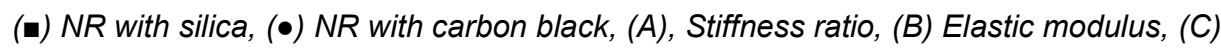
Tensile strength, (D) Elongation at break ${ }^{41}$. 


\subsection{Other Factors Influencing Silica-Filled Rubber Properties}

\subsubsection{EFFECT OF AdDITIVES}

Silanol groups of silica react readily with oxygen- or nitrogen-containing compounds such as glycol, water, alcohols, amines, divalent metal salts and with each other. A soluble zinc compound, e.g. zinc-stearate reacts with silanol groups of silica, and this reaction competes with the zinc activating functions. Soluble zinc ions which are produced from reaction of zinc oxide and fatty acids during compounding are the key for accelerator activation. As presented earlier in Figure 2.25, zinc ions become securely bound to one or two silanol groups, and result in reduced silica network formation as well as decrease in filler to rubber bonds. Loss of soluble zinc from its cure activating function leads to reductions in cure rate, mono and disulfides crosslinks and high strain modulus.

The interaction of silica with glycols or amines through hydrogen bonding provides a buffer layer to reduce the silica-zinc reaction. The addition of glycol alters the character of silica surfaces from hydrophilic to partially hydrophobic ${ }^{77}$. The silanol groups $(\mathrm{Si}-\mathrm{OH})$ on the silica surface may bind to the hydroxyl groups of ethylene glycol through a condensation reaction as shown in Figure 2.41. The interaction between ethylene glycol hydroxyl groups and hydrogen of silica surface silanol groups by means of hydrogen bonds is also possible: Figure 2.42.
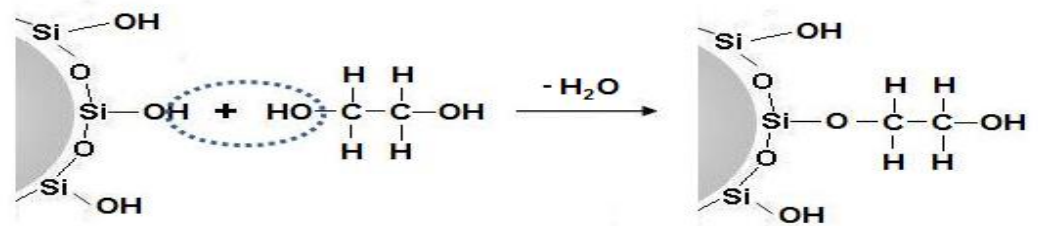

Figure 2.41 Reaction between silanol group of silica and hydroxyl group of ethylene glycol via condensation reaction
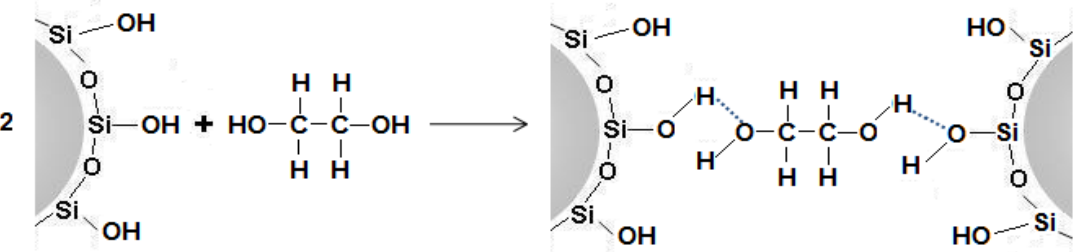

Figure 2.42 Interaction between silanol group of silica and hydroxyl group of ethylene glycol via hydrogen bonding 
One drawback in using silica filler is its effect on the sulfur curing vulcanization. The acidic nature of silanol groups has a retarding or deactivating effect on sulfur curing. The use of silica also results in differences in cure behavior and decreased crosslinking rate when compared with conventional carbon black filler ${ }^{78}$. Polyethylene glycol (PEG) is the most frequently used additive due to its cure acceleration as shown in Table 2.14. Addition of PEG is known to prevent the adsorption of vulcanizing agent on the surface of silica by coating the silica surface ${ }^{78}$. PEG also eliminates the loss in cure rate which occurs during remilling of silica-reinforced natural rubber.

Table 2.14 Cure activation by polyethylene glycol

\begin{tabular}{lccc}
\hline & \multicolumn{3}{c}{ PEG (3350), phr } \\
& $\mathbf{0}$ & $\mathbf{2}$ & $\mathbf{4}$ \\
\hline Cure time, t90 at 144으, min & 34 & 19 & 16 \\
Original & 42 & 21 & 16 \\
Remilled & 51 & 41 & 39 \\
Mooney viscosity, ML[1+4] & 59 & 66 & 60 \\
Hardness, Shore A & 32 & 29 & 22 \\
Heat build-up, ㅇ & \multicolumn{3}{c}{} \\
\hline Ingredients: NR-100, 150m²/g N2SA Silica-30; sulfur-2.8, MBS-1.5, DPG-0.3, ZnO-5, \\
Stearic acid-3 (phr).
\end{tabular}

Besides PEG, other materials suitable for buffering include triethanolamine (TEA), glycerol, and diethyleneglycol (DEG). Comparisons among PEG, DEG and TEA, on equal parts basis, indicate that PEG gives $30 \%$ faster curing than DEG, but slower than TEA. Pal and $\mathrm{De}^{55}$ have found that ethylene glycol (EG) is a weak activator as compared to TEA. TEA is an alkaline substance and therefore neutralizes the acidity of the silanol groups. Based on the extent of reinforcement determined from the Cunneen and Russell equation (eq. 2.7), the $v_{r o} / v_{r f}$ is close to 1: Figure 2.43. The relative activating power of different system is summarized as follows:

$$
\text { No additive }<\text { EG }<\text { TEA }<\text { TESPT }
$$

In their work, Pal and De have measured the extent of silica dispersion using the Lee equation ${ }^{55}$. The Lee equation was initially proposed to correlate the moduli with filler loading, filler morphology and rubber-filler interaction in carbon black compounds ${ }^{79}$ : 


$$
L=\eta_{r}-M_{r}
$$

Where $\eta_{r}=T_{\min , F} / T_{\min , G}$ and $M_{r}=T_{\max , F} / T_{\max , G \text {. }}$

$T_{\min }$, is the minimum torque and $T_{\max }$ is the maximum torque of the cure curve of a mix and $G$ and $F$ refer to gum and filled mix, respectively. From the plot of $L$ against loading of silica, the order of dispersion of filler was determined. The lower the value of $L$ at the particular loading, the higher is the dispersion: Figure 2.44.

In tire tread compounds, dithiophosphates were found suitable replacements for diphenyl guanidine (DPG) as secondary accelerator, which offer advantages of nonnitrosamine generating accelerator systems and non-toxic. The use of dithiophosphate with sulfenamide type accelerators in S-SBR/NR-based silica compounds gives synergistic effects and results in improved reversion resistance and dynamic heat stability ${ }^{80}$.

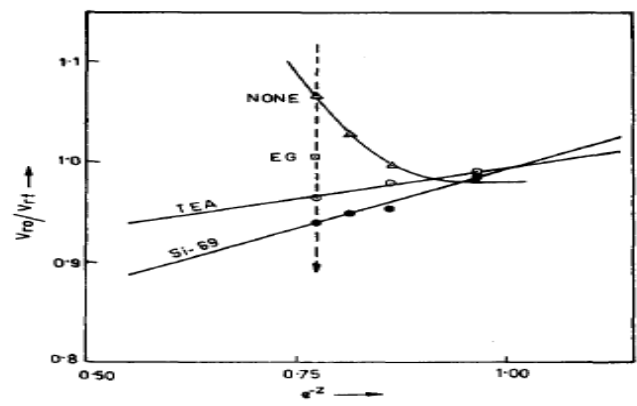

Figure 2.43 Plot of $v_{r o} / v_{r f}$ against $e^{-Z}$ (Cunneen and Russell equation) for silica vulcanizates in the presence of activators (EG and TEA) and coupling agent, Si-6950

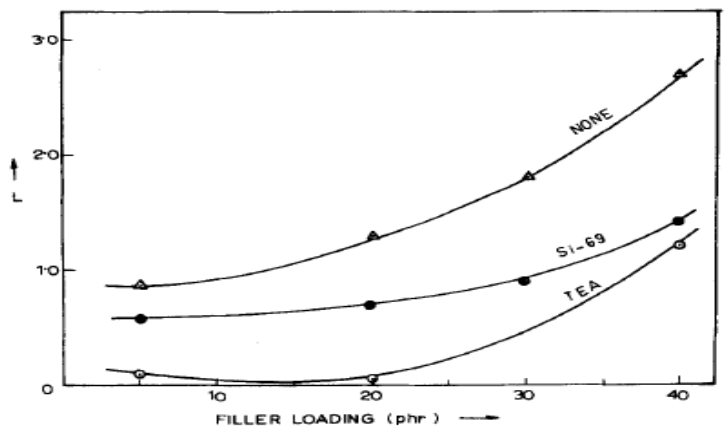

Figure 2.44 Variation of $L$ (Lee parameter) with filler loading ${ }^{550}$

\footnotetext{
${ }^{\circ}$ Reproduced with permission from Rubber Chemistry and Technology. Copyright $@(1982)$, Rubber Division, American Chemical Society, Inc
} 
An optimization in the method of incorporating silica filler in rubber compounds is disclosed with the use and ordered addition of both dispersing and coupling agents during the rubber mixing process ${ }^{81}$. A quaternary ammonium compound is used as dispersing agent for silica in the rubber matrix. The dispersing agent is used in combination with coupling agent in the rubber tread compound to provide improvement in the properties relating to wear, wet traction and rolling resistance.

\subsubsection{EfFect Of Free WATER / MOISTURE CONTENT}

The free water or moisture content in silica reflects its hydrophilic nature and tendency to adsorb moisture to attain equilibrium with relative humidity of its environment. Silanol groups are very unstable and the equilibrium is towards forming the siloxane groups ${ }^{82}$. Hence, siloxane and silanol groups establish an equilibrium depending upon humidity level. The normal moisture content of silica is in a range of 5 to $7 \%$. As illustrated in Figure 2.45, the larger particle aggregates are reduced with dry silica (moisture content less than $1 \%$ ). The average diameter size of the larger aggregates is reduced from 100 to $70 \mathrm{~nm}$ with dry silica. Hewitt has demonstrated that the use of dry silica in the compound has an effect to increase the viscosity, reduce the hardness and increase high-strain modulus: Table $2.15^{27}$. The smaller aggregates, together with the removal of the free water barrier are responsible for the increase in $300 \%$ modulus and abrasion resistance. It is also noted that the viscosity reduces when silica is treated with silane indicating the effect of loss in hydrogen bonded structures.

Table 2.15 Effect of moisture content on NR-silica compound ${ }^{27}$

\begin{tabular}{llll}
\hline \multicolumn{1}{c}{ Silica (160 $\mathbf{~ m}^{\mathbf{2}} \mathbf{g}$ ) } & A & B & C \\
\hline Moisture content (\%) & 6.0 & 0.2 & 0.2 \\
Loading (phr) & 30 & 28 & 28 \\
Mercapto silane (phr) & - & - & 1 \\
ODR, MH-ML, dNm & 18 & 16 & 22 \\
ML (1+4) @100ㄷ & 66 & 90 & 74 \\
Hardness (durometer) & 60 & 57 & 53 \\
M300, MPa & 6.9 & 7.8 & 9.2 \\
Elongation at break, \% & 495 & 490 & 430 \\
Rebound resilience, \% & 84 & 87 & 88 \\
SEM predominat silica size range in vulcanizates, nm & $30-100$ & $30-70$ & $30-50$ \\
\hline Ingredients: SMR CV60-100, ODPA-1, Paraffinic oil-3, Dicumyl peroxide-2.4 & \\
\hline
\end{tabular}



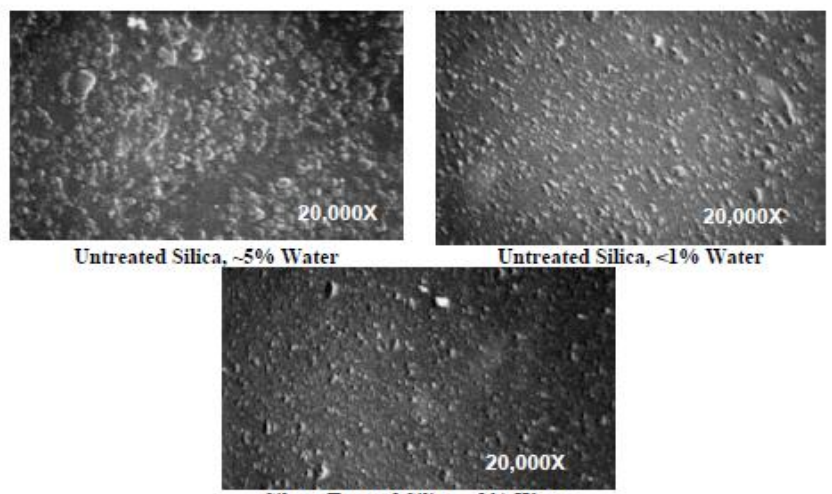

Silane-Treated Silica, $<1 \%$ Water

Figure 2.45 Effect of moisture content on aggregate size of silica in $N R^{27 p}$.

\subsubsection{Effect of Particle Size of Silica}

Particle size has an influence on compound properties. Silicas with smaller particles (high surface area) produce small aggregates, whilst large particles produce large aggregates. Figure 2.46 shows the electron micrographs of three types of silica with different surface area compounded at $30 \mathrm{phr}$. It can be seen that the NR compound containing silica with average primary particle of 20 nanometer $(\mathrm{nm})$ has aggregates of 40 to 100 nanometers in diameter, whilst the compound with silica of $75 \mathrm{~nm}$ primary particle gives larger aggregates of $1 \mu \mathrm{m}$ in size.

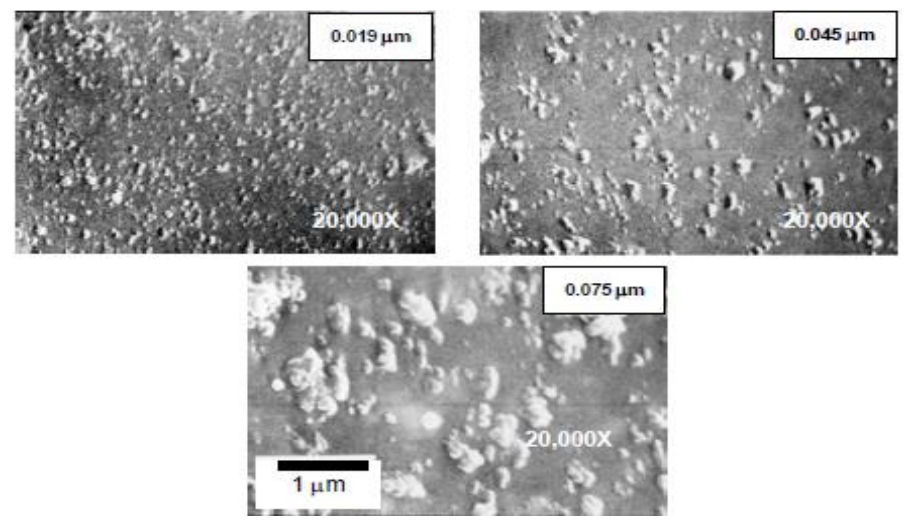

Figure 2.46 Silica primary particle and aggregates sizes in NR compound (30 phr) ${ }^{27 p}$.

\footnotetext{
${ }^{\mathrm{P}}$ Reprinted from Compounding Precipitated Silica in Elastomer, N. Hewitt, Chapter 1: Silica as A Reinforcing Filler, 1-23, Copyright (2007), with permission from Elsevier
} 
Silica generally produces higher Mooney viscosity in rubber mixes than carbon black of comparable size: Table 2.16. However, the difference between the fillers becomes less pronounced as the average filler particle size increases. As shown in Table 2.17, at larger particle size $(40 \mathrm{~nm})$ the silica and carbon black produce similar viscosities ${ }^{83}$.

Table 2.16 Mooney viscosity for carbon black- and silica-filled compounds (50phr) ${ }^{45}$

\begin{tabular}{lcccc}
\hline Filler & $\begin{array}{c}\mathrm{N}_{2} \text { Surface Area } \\
\left(\mathrm{m}^{2} / \mathrm{g}\right)\end{array}$ & $\begin{array}{c}\text { DBPA } \\
(\mathrm{mL} / 100 \mathrm{~g})\end{array}$ & SBR1500 & $\mathrm{NR}$ \\
\hline CB N110 & 140 & 113 & 104 & 52 \\
Silica & 130 & 231 & 201 & 93 \\
\hline
\end{tabular}

Table 2.17 Mooney viscosity for carbon black- and silica-filled NR compounds ${ }^{83}$

\begin{tabular}{lccc}
\hline \multicolumn{3}{c}{ Loading, phr } & \multicolumn{3}{c}{$\mathrm{ML}(1+4)$ at $100^{\circ} \mathrm{C}$} \\
\hline Carbon Black N550, (particle size $45 \mathrm{~nm})$ & 40 & 60 & 70 \\
Silica HiSil-400, (particle size $40 \mathrm{~nm}$ ) & 44 & 61 & 73 \\
\hline
\end{tabular}

\subsection{Summary and Focus of The Project}

This review gave an overview of the work carried out on silica reinforcement of rubbers since it was first introduced as filler. It can be seen that, since at that time NR was the only most common polymer of use, most of the work on silica reinforcement was done with NR. When silica technology for tires was introduced with the use of silane coupling agents, the work still involved NR as base polymer, but gradually was replaced by synthetic rubber. Considerations that need to be taken during mixing silica into rubber compounds are discussed. The good reinforcing behavior of silica in NR is translated into improved tire properties, in particular reduction of rolling resistance and lower heat build-up. The properties of NR and the influence of non-rubber constituents on the strength and network structure of unreinforced NR is well documented. However, little information is available on the effect of non-rubber constituents, particularly proteins on the silica-filled NR compounds. Hence, in this thesis, the postulation on the influence of proteins on the silica-silane reaction in NR compounds will be investigated. 


\subsection{REFERENCES}

1. F. W. Barlow, Rubber Compounding: Principles, Materials and Techniques, Chapter 2: Elastomers: Natural Rubber, M. Dekker, New York, (1988).

2. J. B. van Beilen and Y. Poirier, TRENDS in Biotechnology, 25, 522 (2007).

3. B. Rodgers and W. Waddell, "The Science of Rubber Compounding", in: Science and Technology of Rubber, eds., J.E. Mark, B. Erman and F.R. Eirich, Elsevier Academic Press, USA, (2005).

4. L. Tarachiwin, J. Sakdapipanich and Y. Tanaka, Kautsch. Gummi Kunstst., 58, 115 (2005).

5. A.H. Eng, S. Kawahara and Y. Tanaka, Rubber Chem. Technol., 67, 159 (1993).

6. S. Amnuaypornsri, J. Sakdapipanich, S. Toki and B.S. Hsiao, N. Ichikawa, and Y. Tanaka, Rubber Chem. Technol., 81, 753 (2008).

7. J. Sakdapipanich, J. Biosci. Bioeng., 103, 287 (2007).

8. K. Nawamawat, J. Sakdapipanich, D. Mekkriengkrai, Y. Tanaka, Kautsch. Gummi Kunstst., 61, 518 (2008).

9. L. Tarachiwin, J. Sakdapipanich, K. Ute, T. Kitayama, T. Bamba, E. Fukusaki, A. Kobayashi and Y. Tanaka, Biomacromolecules.,6, 1851 (2005).

10. J. Yunyongwattanakorn, Y. Tanaka, S. Kawahara, W. Klinklai, and J. Sakdapipanich, Rubber Chem. Technol., 76, 1228 (2003).

11. W. Emunds and H. Burhin, FT Rheology, a Tool to Quantify Long Chain Branching in Natural Rubber and its Effects on Mastication, Mixing Behaviour and Final Properties, Rubber Testing, (2007).

12. A.B. Othman and C. Hepburn, Plast. Rub. Compos. Pro., 19, 185 (1993).

13. H.Y. Yeang, S.A.M. Arif, F. Yusof, and E. Sunderasan, Methods, 27, 32 (2002).

14. S. J. Tata, Studies on the Lysozyme and Components of Microhelices of Hevea Brasiliensis Latex, PhD Thesis, University of Malaya, 1980.

15. T. Midgley, Jr, A. L. Henne and M. W. Renoll, J. Am. Chem. Soc., 59, 2501 (1937).

16. E.C. Gregg and J.H. Macey, Rubber Chem. Technol., 46, 47 (1973).

17. S.Amnuaypornsri, A. Nimpaiboon, and J. Sakdapipanich, Kautsch. Gummi Kunstst., 62, 88 (2009).

18. M.J.R. Loadman and W.C. Wake, Analysis of Rubber and Rubber-like Polymers, Kluwer Academic Publishers, the Netherlands, 96 (1988).

19. D.J.C. Salamone, Polymeric Materials Encyclopedia, Vol. 6, CRC Press Inc., 4556 (1996).

20. S. Amnuaypornsri, L. Tarachiwin and J. Sakdapipanich, J. Appl. Polym. Sci., 115, 3645 (2009).

21. P.Rojruthai, L.Tarachiwin, J.T. Sakdapipanich, K.U. Toyonaka and Y. Tanaka, Kautsch. Gummi Kunstst., 62, 227(2009). 
22. P. Rojruthai, L. Tarachiwin, J.T. Sakdapipanich and Y. Tanaka, Kautsch. Gummi Kunstst., 62, 399 (2009).

23. K.T. Cheang, A. R. Rais and K. B. Basir, Deproteinised Natural Rubber (DPNR), Malaysian Rubber Board Monograph No. 10, C\&D Trading, Kuala Lumpur, (2003).

24. H.L. Brantley and G.L. Day, presented at ACS Rubber Division Meeting, New York, USA, paper no.33 (1986).

25. W.J. Kern and S. Futamura, presented at ACS Rubber Division Meeting, Quebec, paper no.78 (1987).

26. W. Meon, A. Blume, H.-D, Luginsland and S. Uhrlandt, in Rubber Compounding Chemistry and Applications, (Ed.: B. Rodgers) Marcel Dekker Inc., New York, USA (2004).

27. N. Hewitt, Compounding Precipitated Silica in Elastomers, William Andrew Publishing, New York, USA (2007).

28. F. Thurn and S. Wolff, Kautsch. Gummi Kunstst.,28, 733 (1975).

29. A. Blume, ACS Rubber Division Meeting, Spring, Chicago, USA, paper no.73 (1999).

30. L. R. Evans and W. H. Wadell, Kautsch. Gummi Kunstst., 48, 718 (1995).

31. M.-J. Wang, S. Wolff and J.-B Donnet, Rubber Chem. Technol., 64, 559 (1990).

32. S. Wolff and M.-J. Wang, Rubber Chem. Technol., 65, 329 (1991).

33. M.-J. Wang and S. Wolff, Rubber Chem. Technol., 65, 715 (1991).

34. Union Carbide, (1991) Organofunctional Silanes, Union Carbide Chemicals and Plastics Company Inc. USA.

35. P. E. Cassidy and B.J. Yager, J. Macromol. Sci--Revs. Polymer Technol., D1, 1 (1971).

36. M. W. Ranney and C.A. Pagano, Rubber Chem. Technol., 44, 1080 (1971).

37. E. P. Plueddemann, Silane Coupling Agents, Plenum Press, New York and London, Second Edition (1991).

38. P.H Sandstrom, R.R. Smith and K.J. Pyle, EP 0989161 A1, The Goodyear Tire \& Rubber Company (1999).

39. S. Wolff, Tire Sci. and Technol., 15 (4), 276 (1987).

40. A. ten Brinke, Silica Reinforced Tire Rubbers, Ph.D Thesis University of Twente, Twente University Press, Enschede, the Netherlands (2002).

41. L.A.E.M. Reuvekamp, Reactive Mixing of Silica and Rubber for Tyres and Engines Mounts, Ph.D Thesis University of Twente, Twente University Press, Enschede, the Netherlands (2003).

42. W. Dierkes, Economic Mixing of Silica-Rubber Compounds, Ph.D Thesis University of Twente, Print Partners Ipskamp, Enschede, the Netherlands (2005).

43. S. Mihara, Reactive Processing of Silica-Reinforced Tire Rubber, Ph.D Thesis University of Twente, Ipskamp Drukkers B.V, Enschede, the Netherlands (2009). 
44. J.W.M. Noordermeer and W.K Dierkes, in Rubber Technologist's Handbook, Vol.2, (Eds.: J. White, S.K. De and K. Naskar), Smithers Rapra Technology, Shawbury, Shrewsbury, Shropshire, UK (2008).

45. S. Wolff, Kautsch. Gummi Kunstst., 34, 280 (1981).

46. E-H. Tan, S. Wolff, M. Haddeman, H.P. Grewatta and M-J. Wang, Rubber Chem. Technol., 66, 594 (1993).

47. H. Westlinnings and S. Wolff, Kautsch. Gummi Kunstst., 19, 470 (1966).

48. S. Wolff, Kautsch. Gummi Kunstst., 23, 7(1970).

49. S. Wolff, Rubber Chem. Technol., 69, 325 (1996).

50. A.R. Payne and R.E. Whittaker, Rubber Chem. Technol., 44, 440 (1971).

51. H-D. Luginsland, J. Fröhlich and A. Wehmeier, Rubber Chem. Technol., 75, 563 (2001).

52. R. Mukhopadhyay and S.K. De, Rubber Chem. Technol., 52, 263 (1979).

53. G. Kraus, Rubber Chem. Technol., 37, 6 (1964).

54. B.B. Boonstra, Polymer, 20, 691(1979).

55. P.K. Pal and S. K. De, Rubber Chem. Technol., 55, 1370 (1982).

56. M. L. Kralevich and J.L. Koenig, Rubber Chem. Technol., 71, 300 (1998).

57. S. Cook, A.V. Chapman, A.J. Tinker and L. Oleksik, Joint conference of the ACS Rubber Division and IRC 2007, Cleveland, Ohio, 16-18 October, paper no 122 (2007).

58. A. Kato, S. Kohjiya and Y. Ikeda, Rubber Chem. Technol., 80, 690 (2007).

59. F. Thurn, K. Burmester, J. Pochert and S. Wolff, US Patent 3,873,489, Degussa(1975).

60. S. Wolff, Kautsch. Gummi Kunstst., 30, 516 (1977).

61. U. Görl, J. Munzenberg, H.-D. Luginsland and A. Muller, Kautsch. Gummi Kunstst., 52, 588 (1999) .

62. A. Ansarifar, R. Nijhawan, T. Nanapoolsin and M. Song, Rubber Chem. Technol., 76, 1290 (2003).

63. A. Ansarifar, L.K. Chong, J. Zhang, A. Bell and R.J. Ellis, Int. J. Adhes. Adhes., 23, 177(2003).

64. A. Ansarifar, S.F. Shiah and M. Bennett, Int. J. Adhes. Adhes., 26, 454 (2006).

65. A. Ansarifar, N. Ibrahim and M. Bennett, Rubber Chem. Technol., 78, 793 (2005).

66. L. Bokobza and O. Rapoport, J. Appl. Pol. Sci., 85, 2301(2002).

67. L. Bokobza and J-P. Chauvin, Polymer, 46, 4144 (2005).

68. H.S. Chung and S.K. Dae, Polym. Adv. Technol., 19, 1062 (2008).

69. S. Wolff, Rubber Chem. Technol., 55, 967 (1982).

70. H.-D. Luginsland, presented at $11^{\text {th }}$ International SRC Meeting, Puchov, Slovakia (1999).

71. H.-D. Luginsland, and A. Hasse, ACS Rubber Division Meeting, Fall, Ohio, USA, paper no. 34 (2000). 
72. L.A.E.M. Reuvekamp, J.W. ten Brinke, P.J. van Swaaij and J.W.M. Noordermeer, Rubber Chem. Technol., 75, 187 (2002).

73. R. Rauline, FR Patent 9102433, Compagnie Generale des Etablissements Michelin-Michelin \& Cie (1992).

74. Y. Chevallier and J.-Cl. Morawski, EU Patent 0157703, Rhone-Poulenc Chimie (1984).

75. S. Wolff, Kautsch. Gummi Kunstst., 41, 674(1988).

76. S. Wolff, ACS Rubber Division Meeting, Cleveland (USA), paper no. 6 (1979).

77. T. Jesionowski and A. Krysztafkiewicz, Appl. Surf. Sci., 172,18 (2001).

78. D. D. Parker and J. L. Koenig, J. Adhesion, 73, 299 (2000).

79. B.L. Lee, Rubber Chem. Technol., 52, 1019 (1979).

80. H.-M., Issel, L., Steger, A. Bischoff, Kautsch. Gummi Kunstst., 58, 529 (2005).

81. G. L. Day, US Patent 6,025,428 Hankook Tire Co. Ltd (2000).

82. G. Akovali, in The Interfacial Interactions in Polymeric Composites, (Eds.H. Ishida), Kluwer Academic Publishers, Netherland, 69 (1993).

83. M.P. Wagner, Rubber Chem. Technol., 49, 703(1966). 


\section{Chapter 3}

\section{The Influence of Processing Temperature on Silica Reinforced Natural Rubber}

The thermal history and in particular the mixing dump temperature is a parameter of paramount importance in mixing rubber and silica with a silane coupling agent in order to achieve proper silanization of silica and to avoid premature scorch reactions. In this chapter, the influence of mixing dump temperature on the performance of silica reinforced Natural Rubber (NR) is investigated. The investigation also includes the effect of non-rubber constituents, primarily proteins in NR, by using deproteinized Natural Rubber (DPNR) and synthetic polyisoprene (IR). The vulcanization properties and rubber-to-filler interactions of silica reinforced $N R$ in presence and absence of a silane coupling agent are highlighted. With increasing mixing dump temperature, the silanization reaction between silica and silane coupling agent proceeds further. At sufficiently high dump temperature, filler-filler interactions in the NR-silica compounds are reduced and silica-rubber interaction improved as evidenced by a drop in the Payne effect and increment in chemically bound rubber. It is demonstrated that $N R$ and IR compounds mixed till above the optimum dump temperature exhibit cure reversion and reduction in tensile properties. On the other hand, DPNR-silica vulcanizates show slightly more constant physical properties. $^{\text {a }}$

\footnotetext{
${ }^{a}$ This chapter is accepted for publication in Rubber Chemistry and Technology, in press (2013)
} 


\subsection{INTRODUCTION}

Compared to carbon black filled materials, mixing of silica compounds involves many difficulties due to the large polarity difference between silica and rubber. A bifunctional organosilane such as bis(triethoxysilyl propyl) tetrasulfide (TESPT) or its disulfide equivalent is commonly used as coupling agent ${ }^{1}$ in enhancing the compatibility of silica and rubber, by chemically modifying silica surfaces and eventually creating a chemical link between silica aggregates and the rubber chains: Figure 3.1. Complications arise during mixing silica compounds as several chemical reactions need to take place, all at their appropriate time slots during rubber processing, namely the silica and silane reaction or silanization, silanerubber coupling and crosslinking among the rubber chains ${ }^{2}$. The highly-dispersible silica technology, as it is used today, employs mainly solution-polymerized synthetic rubber, and is still not fully commercially feasible with Natural Rubber (NR) ${ }^{3}$. It was postulated that nonrubber constituents contained in NR such as proteins compete with the coupling agent for reaction with the silica during mixing, so disturbing its reinforcement action.

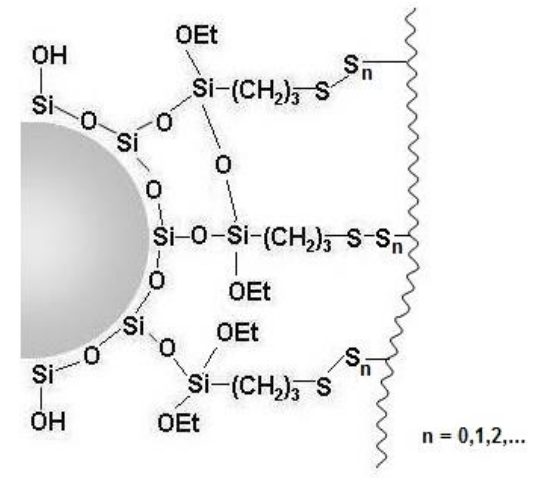

Figure 3.1 Silica-silane-rubber coupling

$\mathrm{NR}$ is a unique material with outstanding properties, such as high tensile strength, good resilience and low heat build-up, superior to synthetic polyisoprene (IR). NR derived from Hevea Brasiliensis latex contains about 3-5\% of non-rubber constituents ${ }^{4}$, essentially proteins and phospholipids, while in contrast IR has none. The structure of a linear NR chain consists of a long sequence of 1000 - 3000 cis-1,4 isoprene units, two trans-1,4 isoprene units, with $\alpha$ - and $\omega$-chain ends ${ }^{5}$. The $\alpha$ - and $\omega$-terminals are associated with phospholipids and proteins respectively ${ }^{6-7}$, and are presumed to play a part in the branching and gel formation in $\mathrm{NR}^{8}$. IR, synthetically produced from isoprene, may contain between 90-98 wt $\%$ cis-1-4 configuration, and the rest are trans-, 1,2- and 3,4- added isomers. It is 
absolutely free of proteins and phospholipids, but due to the absence of the almost $100 \%$ of cis-1-4 configuration it commonly shows little strain crystallization with consequent lower green strength and hence lower mechanical properties ${ }^{9}$.

Gregg and Macey have demonstrated that the insoluble non-rubber constituents in $N R$ also account for the differences in properties between compounded NR and IR ${ }^{10}$. These non-rubber materials, mostly proteins, are responsible for the higher modulus, faster scorch time and higher tear strength of NR. The protein is postulated to act as a reinforcing filler at low concentrations (3-4 wt.\%) and as a cure activator. Othman and Hepburn have shown that the presence of proteins did not significantly affect the elastic modulus of a rubber vulcanizate $^{4}$. However, the presence of its hydrolyzed constituents, amino acids, gave a marked increase in the modulus of vulcanizates.

The green strength of NR has always been ascribed to originate from proteins in NR. On the other hand, the removal of proteins in NR through deproteinization gives green properties similar to $\mathrm{NR}^{5,11}$; the removal of both proteins and phospholipids in $\mathrm{NR}$ by combined deproteinization and transesterification results in rubber with green strength comparable to that of $\mathrm{IR}^{11-13}$. Conversely, a simple addition of extracted proteins and/or phospholipids to the treated NR (after deproteinization and lipase treatment), did not improve the tensile properties, as it is not sufficient to revive the naturally occurring network in $\mathrm{NR}^{7}$. Consequently, the superior stress-strain behavior and the strain-induced crystallization of NR in both, unvulcanized and vulcanized form, must be from its characteristic network structure, which originates from the bonding of proteins and phospholipids with the terminal chain ends of the natural polyisoprene ${ }^{7}$. In a synchrotron $x$ ray study, the difference in the strength and strain-induced crystallization of vulcanized NR and IR is reported to originate from the pseudo or naturally occurring network ${ }^{14}$.

The thermal history and in particular the mixing dump temperature has been shown to be the parameter of paramount importance in mixing silica and rubber in presence of TESPT as coupling agent ${ }^{15-16}$. Wolff has reported that temperature has a more dominant effect than time in the silica-TESPT reaction ${ }^{17}$. In order to achieve a sufficient degree of silanization, the temperature during mixing should be between 150 and $160^{\circ} \mathrm{C}$. However, above $160^{\circ} \mathrm{C}$ either the coupling agent starts to prematurely react with the rubber matrix or the TESPT starts to donate sulfur; both result in pre-scorch of the compound. Reuvekamp et al. demonstrated that a mixing time of at least 10 minutes at $150^{\circ} \mathrm{C}$ is necessary to ensure complete coupling of the silica and the silane, and that the reaction between the silica and the silane takes place primarily during the first mixing $\operatorname{step}^{15}$. 
In the present work, the influence of mixing dump temperature on properties of silica reinforced NR is investigated. The effect of non-rubber constituents, particularly proteins, on silica reinforcement is highlighted in the presence and absence of coupling agent. NR is compared with purified NR from deproteinization (DPNR) as well as with synthetic polyisoprene (IR).

\subsection{EXPERIMENTAL}

\subsubsection{Materials}

Standard Malaysian Natural Rubber (SMR20, 100 MU, ML[1+4]@100C) and Deproteinized Natural Rubber (Pureprena, $70 \mathrm{MU}, \mathrm{ML}[1+4] @ 100^{\circ} \mathrm{C}$ ) were provided by the Malaysian Rubber Board. The nitrogen contents of SMR20 and Pureprena are 0.2 and 0.07 respectively. It is generally accepted that the conversion factor from nitrogen content to protein content is $6.25^{4,18}$. Synthetic polyisoprene (Nipol IR 2200, 98\% cis-1,4 content, 82 $\mathrm{MU}, \mathrm{ML}[1+4] @ 100^{\circ} \mathrm{C}$ ) was supplied by Zeon Corporation, Japan. Highly dispersible silica, Ultrasil 7005 (CTAB surface area :164 $\mathrm{m}^{2} / \mathrm{g}$ ), and silane coupling agent, bis (triethoxysilylpropyl) tetrasulfide (TESPT), from Evonik were used. All the ingredients were used as obtained from the respective sources. The compound formulation is based on a typical carbon black based truck tire tread recipe with the black replaced by silica as shown in Table 3.1.

Table 3.1 Compound formulation

\begin{tabular}{lcc}
\hline \multicolumn{1}{c}{ Ingredients } & Source & Amount (phr) \\
\hline Rubber & vary & 100 \\
Silica Ultrasil 7005 & Evonik & 55 \\
Silane, TESPT & Evonik & 5 \\
Zinc oxide (ZnO) & Sigma Aldrich & 2.5 \\
Stearic acid (St.A) & Sigma Aldrich & 1 \\
Treated distillate aromatic extract (TDAE) oil & Hansen \& Rosenthal & 8 \\
2,2,4-trimethyl-1,2-dihydroquinoline (TMQ) & Flexsys B.V. & 2 \\
Sulfur & Sigma Aldrich & 1.4 \\
N-cyclohexyl-2-benzothiazyl sulfenamide (CBS) & Flexsys B.V. & 1.7 \\
Diphenyl guanidine (DPG) & Flexsys B.V. & 2 \\
\hline
\end{tabular}

\subsubsection{Sample Preparation}

The compounds were mixed in 2 steps. The first step was done using a laboratory internal mixer Brabender Plasticoder 350S lab station with a capacity of $390 \mathrm{ml}$. The fill factor of the 58 
mixer was fixed at $70 \%$ and the rotor speed used was $60 \mathrm{rpm}$. The starting temperature of the mixing chamber and rotor was varied from 70 to $120^{\circ} \mathrm{C}$ in order to obtain variable temperature histories and dump temperatures. A typical fingerprint of masterbatch mixing indicating the dump temperature is represented in Figure 3.2. The dump temperature was recorded from the mixer control unit and the value taken as it appeared from the WinMix software. After mixing for 14 minutes, the batches were sheeted out on a Schwabenthan Polymix $80 \mathrm{~T} 80 \times 300 \mathrm{~mm}$ two-roll mill, with a tight nip with 10 passes to improve the dispersion of silica. After 24 hours, the curatives were mixed on the luke-warm two-roll mill in the second step. The mixing procedure is presented in Table 3.2

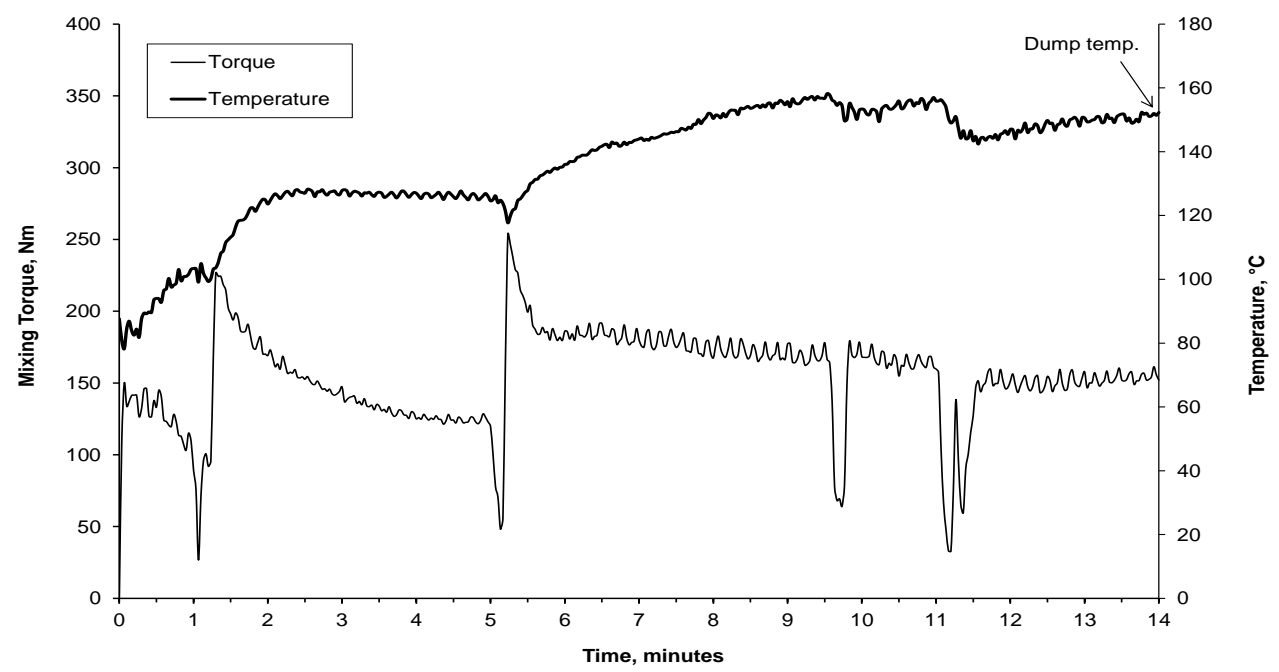

Figure 3.2 Typical fingerprint of the masterbatch mixing of the first mixing stage in the internal mixer showing the dump temperature.

Table 3.2 Mixing Procedure

\begin{tabular}{cll}
\hline & \multicolumn{1}{c}{ Step 1 } & \multicolumn{1}{c}{ Step 2 } \\
\hline Time (minute) & Action & All curatives \\
0.00 & Add rubber, mastication & (DPG, CBS and S) \\
1.00 & Add $1 / 2$ silica, $1 / 2$ silane & were mixed on \\
5.00 & Add $1 / 2$ silica, $1 / 2$ silane, oil & two-roll mill \\
9.00 & Sweep & \\
11.00 & Add ZnO, St.A, TMQ & \\
14.00 & Dump & \\
\hline
\end{tabular}




\subsubsection{Sample Analyses}

Mooney viscosity was measured at $100^{\circ} \mathrm{C}$ with a Mooney viscometer 2000E (Alpha Technologies) using the large rotor $(\mathrm{ML}(1+4))$ for compounds and the small rotor for the masterbatches $(\mathrm{MS}(2+4))$. Vulcanization curves were measured using a Rubber Process Analyzer (RPA 2000) from Alpha Technologies, under conditions of $0.833 \mathrm{~Hz}$ and $2.79 \%$ strain over a period of 30 minutes at a temperature of $150^{\circ} \mathrm{C}$. The Payne effect was measured prior and after cure in the RPA 2000 as well. Before cure, the sample was heated to $100^{\circ} \mathrm{C}$ in the RPA and subsequently subjected to a strain sweep at $0.5 \mathrm{~Hz}$. The Payne effect was calculated as the difference between the storage modulus, $G^{\prime}$ at $0.56 \%$ and $G^{\prime}$ at $100.04 \%$ strain. The Payne effect after cure was measured after vulcanization in the RPA 2000 at $150^{\circ} \mathrm{C}$ for 10 minutes and subsequent cooling to $100^{\circ} \mathrm{C}$, making use of the same strain sweep conditions.

Wolff's filler structure parameter, $\alpha_{f}$ was determined from the ratio between the increase in vulcameter torque of the filled compounds and that of the unfilled gum ${ }^{18}$ :

$$
\frac{D_{\max }-D_{\min }}{D_{\max }^{o}-D_{\min }^{o}}-1=\alpha_{f} \frac{m_{f}}{m_{p}}
$$

Where $D_{\max }-D_{\min }$ is the maximum change in torque for the filled rubber, $D^{o}{ }_{\max }-D^{\circ}{ }_{\min }$ is the maximum change in torque for the unfilled gum rubber, $m_{f} / m_{p}$ is the weight ratio of filler to polymer, $\alpha_{f}$ is a filler specific constant which is independent of the cure system and closely related to the morphology of the filler.

The bound rubber content (BRC) measurements were performed on masterbatch samples without curatives by extracting the unbound rubber with toluene at room temperature for seven days in both, normal and ammonia environment. The ammonia treatment of BRC was done to obtain the chemically bound rubber as ammonia cleaves the physical linkages between rubber and silica ${ }^{20-21}$. The amount of BRC ( $g / g$ filler) was calculated by:

$$
B R C(g / g \text { filler })=\frac{w_{d r y}-w_{\text {insol }}}{w_{o} \times \frac{w_{\text {filler }, p h r}}{w_{\text {total }, p h r}}}
$$

Where $w_{0}$ is the initial weight of the sample, $w_{\text {dry }}$ is the dry weight of the extracted sample, $W_{\text {insol }}$ is the weight of insolubles (mainly filler) in the sample, $W_{\text {filler,phr }}$ is the total filler weight in phr and $w_{\text {total, }}$ phr is the total compound weight in phr. The physically BRC was taken as the difference between untreated BRC and ammonia treated BRC. 
Vulcanizates were prepared by curing the compounds for their respective t95 $_{95}$ (time to reach $95 \%$ of torque difference in the curemeter) at $150^{\circ} \mathrm{C}$ using a Wickert laboratory press WLP $1600 / 5^{\star} 4 / 3$ at 100 bar. Tensile properties of the vulcanizates were measured using a Zwick Z020 tensile tester according to ISO-37. The hardness of the cured samples was determined according to DIN-53505. The tan delta (G"/G') at $60^{\circ} \mathrm{C}$ was measured using the RPA 2000 by applying a frequency sweep at $3.49 \%$ strain after first curing in the RPA at $150^{\circ} \mathrm{C}$.

\subsection{ResUlts AND Discussion}

\subsubsection{Compound Processability}

In terms of processability of the masterbatches, NR and DPNR are comparable, but IR shows completely different behavior with lower viscosity. In Figure 3.3, the increase in viscosity of the masterbatches of NR and DPNR with rising mixer dump temperature up to a temperature of $150^{\circ} \mathrm{C}$ is due to the increase in silanization degree of the silica and rubber-tofiller interactions. As more silica is silanized by TESPT when the dump temperature is raised, the viscosity is expected to decrease due to better hydrophobation. However, this is not the case for NR and DPNR, as both the silica-TESPT reaction and TESPT-rubber coupling occur simultaneously. The increase in silica-rubber interactions and silica-TESPTrubber coupling results in restriction of the mobility of rubber chains and consequently an increase of the viscosity. Oppositely, in the case of synthetic polyisoprene IR, the Mooney viscosity is not affected by a dump temperature below $150^{\circ} \mathrm{C}$. In addition, the Mooney of IR is lower than of NR to start with because of the difference in molecular weight and absence of a pseudo network. This demonstrates the different behavior of synthetic rubber as compared to NR as also reported by Kaewsakul and coworkers ${ }^{16}$. With increasing dump temperature, the silanization of silica in the IR masterbatch also increases but molecular motion of the rubber chain is not restricted. It is interesting to see that the viscosity of all masterbatches decreases above their respective optimum dump temperatures. One possible explanation is the molecular chain scission of NR at higher temperatures during mixing, which may even be more pronounced in its synthetic equivalent IR. This thermomechanical degradation of NR and IR results in reduction of their molecular weights and hence lower viscosities.

Once the curatives are added to the compounds, the viscosities of NR and DPNR drop to acceptable levels due to many factors which include the remilling step, silica dispersion, interaction of polar curatives with silica, and reduction of chain entanglements or 
pseudo network of rubber chains. For the NR compounds, the Mooney viscosity is seen to increase with increasing dump temperature up till $150^{\circ} \mathrm{C}$ and becomes constant afterwards.

For the DPNR compounds, a linear increase in Mooney viscosity is observed with increasing dump temperature to a maximum of $160^{\circ} \mathrm{C}$, and the values are also higher than those of NR. The increasing trend observed for NR and DPNR compounds with curative indicate the secondary reaction of silanization still continue even after mixing. However, the IR compound now exhibits higher Mooney viscosities, almost double of those of NR. In addition, the IR compound shows a clear optimum in viscosity at dump temperature of $150^{\circ} \mathrm{C}$. Here the trend of the IR compound is similar to the previously observed trend of the NR and DPNR masterbatches. The rise in the viscosity up to $150^{\circ} \mathrm{C}$ is attributed to the silanization process being accelerated upon addition of amine (DPG) in the second stage of mixing and resulting in more silica-rubber interaction. This indicates that silanization occurs much faster in NR and DPNR as compared to IR, which can be attributed to the non-rubber constituents making the environment more acidic or alkaline and facilitating the silica-TESPT reaction. The decrease in Mooney viscosity for IR above a dump temperature of $150^{\circ} \mathrm{C}$ is most probably due to main chain scission of IR, like for NR.

(a) masterbatches

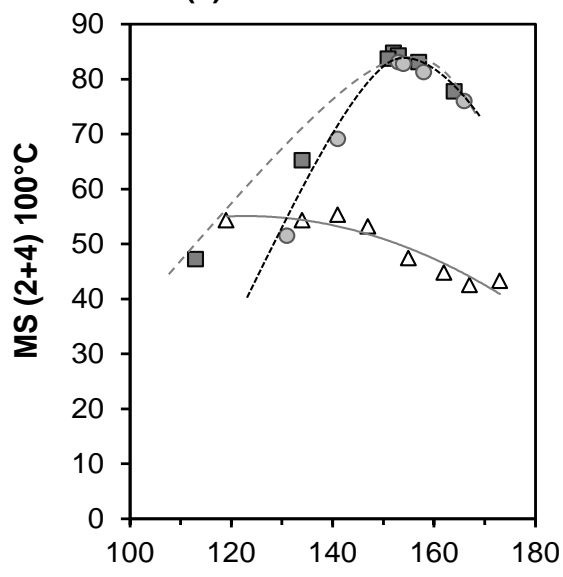

Dump Temperature, ${ }^{\circ} \mathrm{C}$ (b) compounds

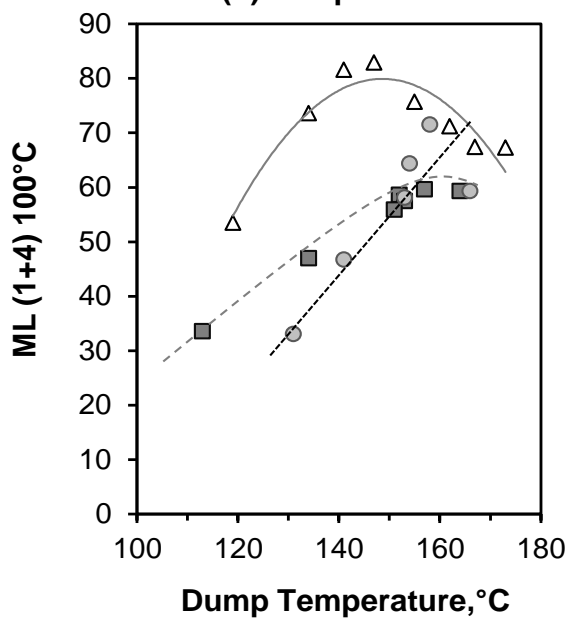

Figure 3.3 Mooney viscosities of : (a) masterbatches after $1^{\text {st }}$ mixing, and (b) compounds after $2^{\text {nd }}$ mill mixing of silica-filled compounds at varying dump temperature: (曰): NR; (•): $\operatorname{DPNR} ;(\Delta): I R$. 


\subsubsection{Effect of silane TESPT on filler flocculation}

Without silane coupling agent, silica develops strong networking in the compounds ${ }^{1,2}$. A high initial torque rise leading to a two-step vulcanization curve is observed in all NR-, DPNR- and IR-silica compounds without silane as illustrated in Figure 3.4(a). The initial torque rise at the beginning of the vulcanization is commonly associated with reagglomeration or flocculation of silica in the compounds ${ }^{22,23}$. The NR compound has a shorter scorch time than the DPNR compound. On the other hand, the IR compound indicates a scorch problem: practically no scorch time at the vulcanization temperature employed. The use of silane TESPT in the silica compound results in far less pronounced silica flocculation as demonstrated by only a small initial torque rise at the beginning of vulcanization: Figure 3.4(b). As compared to the silica compounds without silane, the flocculation tendency of silica in the compounds with TESPT is small due to hydrophobation of the silica surface by TESPT. It is noted that the scorch safety of NR and IR also improves with the use of TESPT.
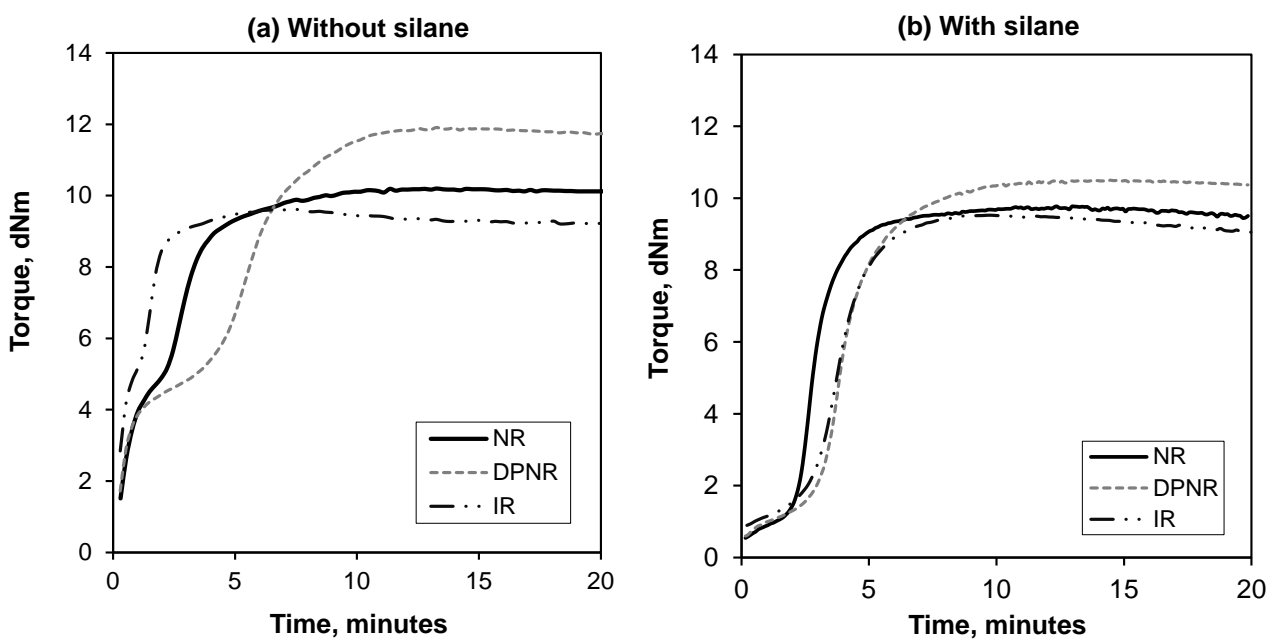

Figure 3.4 Comparison of vulcanization curves at $150^{\circ} \mathrm{C}$ of silica compounds: (a) without silane and (b) with silane

\subsubsection{Vulcanization properties}

The influence of the mixing temperature history, in particular the dump temperature of the first mixing step on the vulcanization behavior of the NR-silica-TESPT compounds as compared to the DPNR-silica-TESPT and IR-silica-TESPT compounds is illustrated in Figures 3.5 and 3.6. The vulcanization behavior of the compounds can be divided 
into three groups according to dump temperature. The first one encompasses the compounds with low dump temperatures (below $150^{\circ} \mathrm{C}$ ), which exhibit pronounced flocculation as evidenced by initial torque rise, high maximum torques and long scorch times. The middle group is represented by the vulcanization curves of the compounds with dump temperatures around $150^{\circ} \mathrm{C}$ to $155^{\circ} \mathrm{C}$, which show no appearance of flocculation, lower maximum torques and shorter scorch times as compared to those of the compounds with low dump temperature. The vulcanization curves of the third group, the compounds with high dump temperatures $\left(>155^{\circ} \mathrm{C}\right)$, display a lower maximum torque and no sign of flocculation. At high mixing temperature, the silanization reaction increases and results in a reduction of silica-silica interaction. However, in the vulcanization curves for these high dump temperatures, there is also clear indication of reversion.
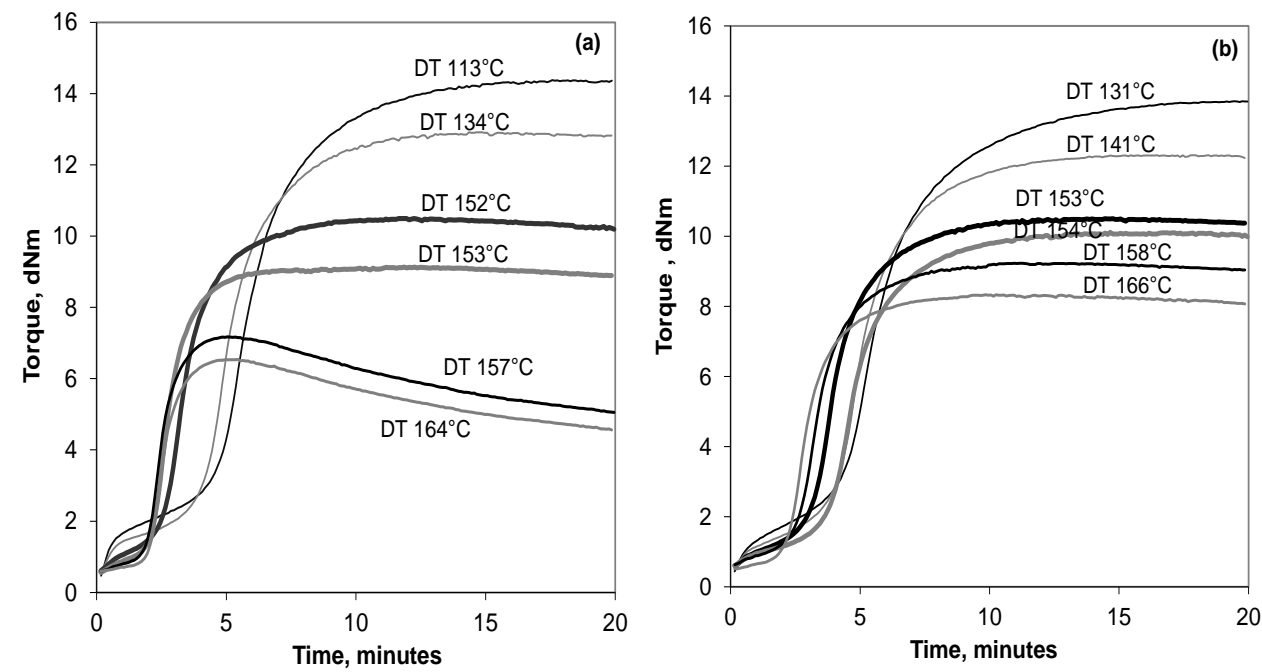

Figure 3.5 Vulcanization behavior at $150^{\circ} \mathrm{C}$ of (a): the NR compounds and (b): DPNR compounds, mixed till different dump temperatures (DT) in the first mixing stage.

The effect of mixing temperature history on vulcanization behavior of the DPNRsilica compound is smaller than for the NR-silica compound. Silica flocculation occurs in the DPNR compound mixed with lowest dump temperature, as also in NR. The maximum torque of the DPNR compound mixed at moderate temperature of around $150^{\circ} \mathrm{C}$ is comparable with the NR compound. Nonetheless, the decrease in maximum torque for the DPNR compound at higher dump temperature is much less compared to the NR-silica compound. The low protein content in DPNR may attribute to better silanization in the compound which gives more filler to rubber linkages. The DPNR-silica compounds with high dump temperature also 
show no sign of reversion as seen in NR compounds. This suggests that a different network structure in the DPNR contributes to the thermal resistance of the cured compound.

In Figure 3.6, the influence of dump temperature is most pronounced for the IRsilica-TESPT compound. The increase in dump temperature reduces the maximum torque of the IR compound very significantly. The vulcanization curves resemble those for NRsilica-TESPT most closely, where the various phenomena are even more pronounced.

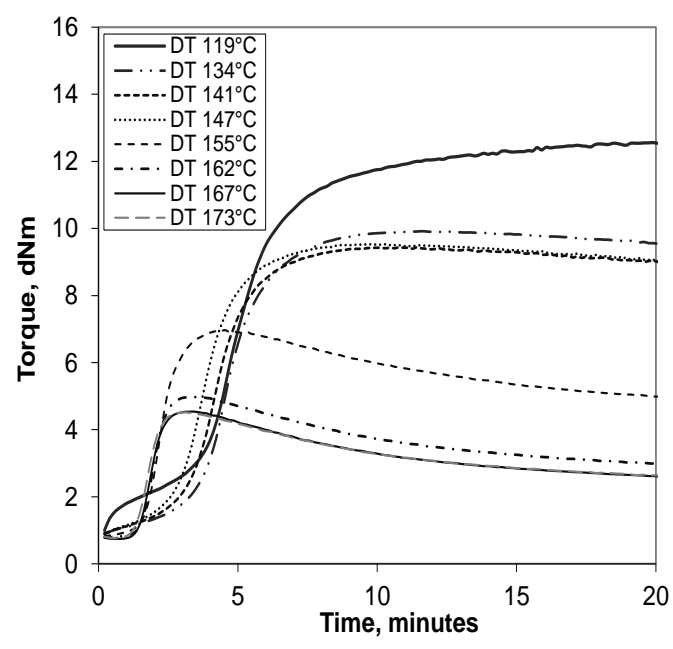

Figure 3.6 Vulcanization behavior at $150^{\circ} \mathrm{C}$ of the IR compound mixed till different dump temperatures $(D T)$ in the first mixing stage.

\subsubsection{Influence of silane TESPT on Payne effect}

Filler-filler interaction is commonly measured by the so-called Payne effect: the drop in storage modulus in a dynamic mechanical test when the strain (deformation) is increased from low $(0.56 \%)$ to a high value $(100 \%)$ at constant frequency and temperature. The storage modulus of filled rubber drastically decreases as strain increases as the result of breakage of physical bonds between filler particles, for example van der Waals interactions, hydrogen bonds and London forces.

The use of silica without silane modification in rubber, mixed till $160^{\circ} \mathrm{C}$ dump temperature, results in a high Payne effect due to strong interaggregate interaction of silica as shown in Figure 3.7. This relates well with the marked flocculation signs in the vulcanization curves of the silica compounds without silane. With TESPT modification, the Payne effect of the silica-filled compounds is greatly reduced as more silica surface is hydrophobized by TESPT, the silica-silica network is disrupted. The Payne effect for 
compounds with silane is observed to decrease in the order of $N R>D P N R>I R$. This points to a relation between the amount of protein and the increase of silica-silica interaction. In other words, it indicates that the hydrophobation of the silica surface by silane is indeed partially hindered due to silica-protein interactions.
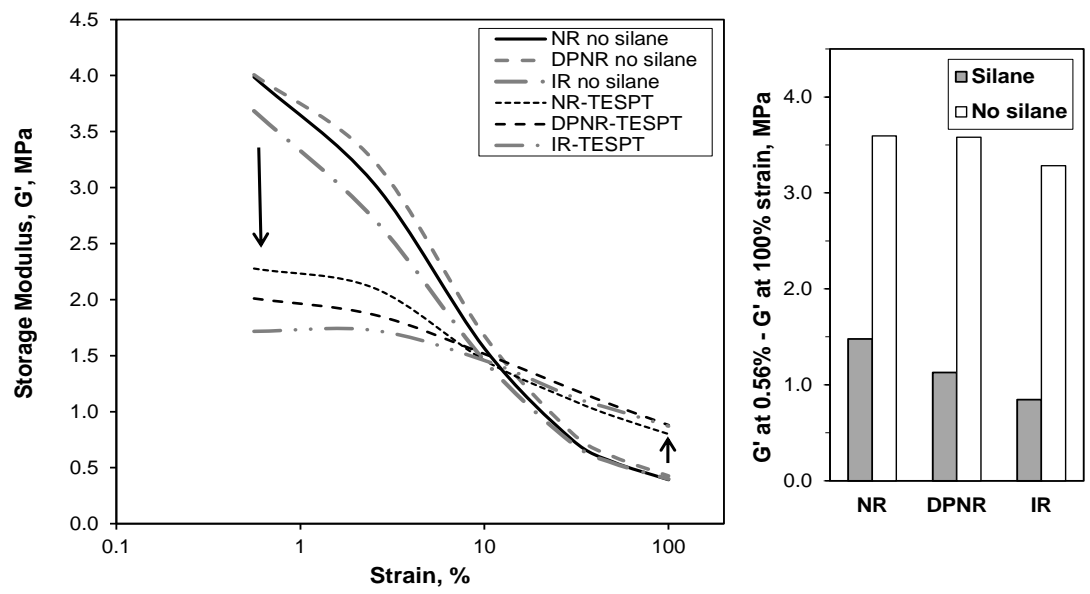

Figure 3.7 Comparison of Payne effect of silica compounds with and without silane

The use of TESPT in silica-filled NR, DPNR and IR also results in higher modulus, G' at high strains as compared to the compounds without silane, as illustrated in Figure 3.7. The values of G' at high strain for all three rubbers do not differ much. This phenomenon is described by Luginsland et al. as the formation of in-rubber structure ${ }^{23}$. The chemical linkages formed by silica-TESPT-rubber coupling result in the immobilization of rubber on the silica surface and within its structure.

A more detailed comparison of filler-filler interaction in NR-silica-TESPT compounds with DPNR-silica-TESPT and IR-silica-TESPT compounds as a function of mixing temperature is shown in Figure 3.8. The Payne effect of all silica-TESPT compounds decreases sharply with increasing dump temperature, as is also seen in synthetic rubber / silica compounds and taken as a sign of reaction and consequent hydrophobation of the silica by the silane coupling agent ${ }^{15-16}$. Before vulcanization, the Payne effect for IR compounds is surprisingly higher as compared to the NR-silica and DPNR-silica compounds. In contrast, after vulcanization, the Payne effect of the IR compound is lower than for the NR- and DPNR-compounds throughout the variation of dump temperature. This indicates that the silanization of silica in IR proceeds slower during mixing than in NR, but is more than compensated after vulcanization. 

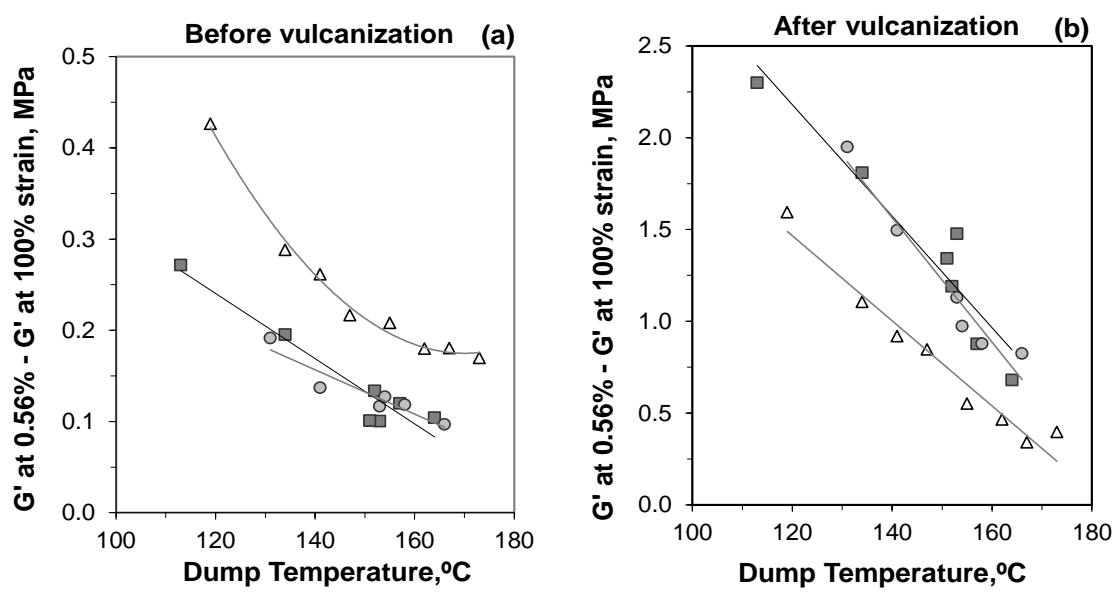

Figure 3.8 Payne effect of silica compounds with silane TESPT as a function of dump temperature: (a) unvulcanized samples; (b) vulcanized samples; (घ): NR; (•): DPNR; $(\Delta): I R$.

\subsubsection{Rubber-to-filler interactions}

Figure 3.9 illustrates the effect of mixing temperature on Wolff's filler structure parameter, $\alpha_{f}$. As observed earlier with the Payne effect, $\alpha_{f}$ is reduced with increasing dump temperature for the NR, DPNR and IR compounds. Better hydrophobation leads to a decrease in silicasilica interaction and consequently results in reduced $\alpha_{f}$. The DPNR compound shows a higher $\alpha_{f}$ than the NR compound, indicating a different type of filler and rubber network in the two compounds. For the synthetic IR compound, the $\alpha_{f}$ value is smaller than for NR. This corresponds with their Payne effects after vulcanization as shown earlier.

The filler to rubber interaction of silica-filled NR with varying protein content can also be judged on basis of the chemically and physically bound rubber content as illustrated in Figure 3.10. The chemically bound rubber of the silica-TESPT compound increases with increasing dump temperature up to $150^{\circ} \mathrm{C}$. This can be explained by the higher rate of silanization with increasing mixing temperature. However, at $150^{\circ} \mathrm{C}$, there is saturation in the amount of TESPT which has reacted and the surface of silica covered. Precipitated silica has about 4 to 5 silanol groups per $\mathrm{nm}^{2}$. Hence, there is no increase in chemically bound rubber for compounds mixed till above $150^{\circ} \mathrm{C}$ dump temperature. Above $150^{\circ} \mathrm{C}$, the chemically bound rubber slowly decreases for NR and DPNR, while for IR it stabilizes. In comparison, DPNR has more chemically bound rubber than NR, particularly at high dump temperature. Meanwhile, the IR compounds exhibit a considerably lower chemically bound rubber as compared to the NR and DPNR compounds. This is due to the slower rate of 
silanization in IR, as could also be taken from the results of the Payne effect before vulcanization above: Figure 3.8(a). Hence, the rubber to filler interaction is also reduced.

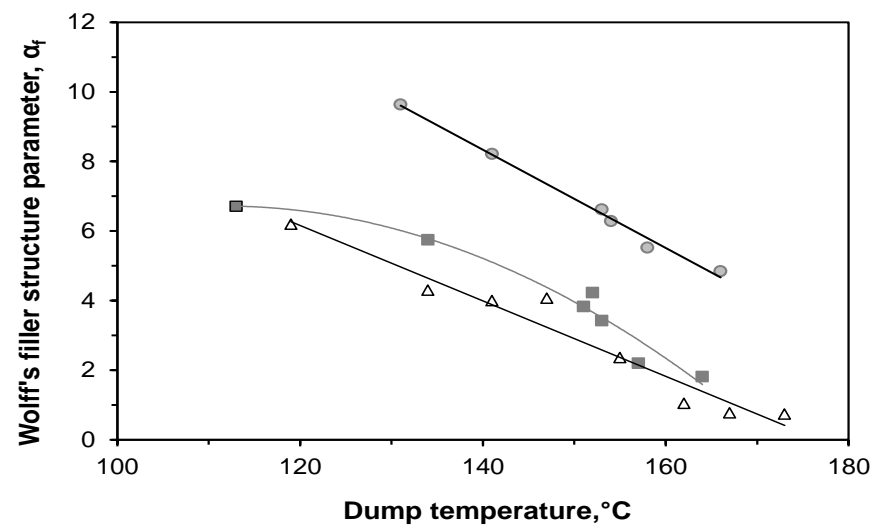

Figure 3.9 Effect of dump temperature on Wolff's filler structure parameter, $\alpha_{f}$ of silica reinforced compounds: (曰): NR; (०): DPNR; $(\Delta): I R$.

(a) Chemically BRC

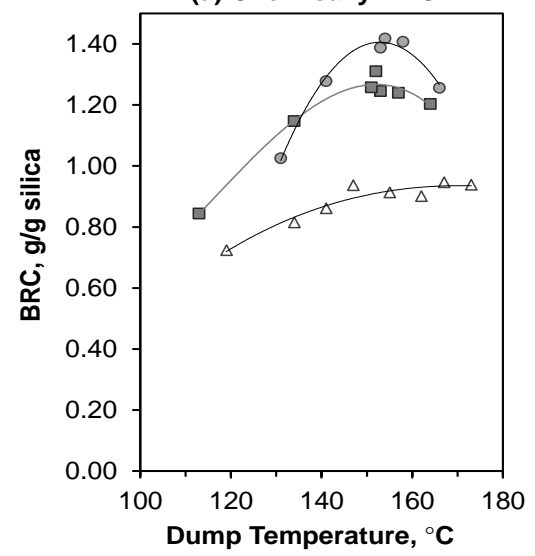

(b) Physically BRC

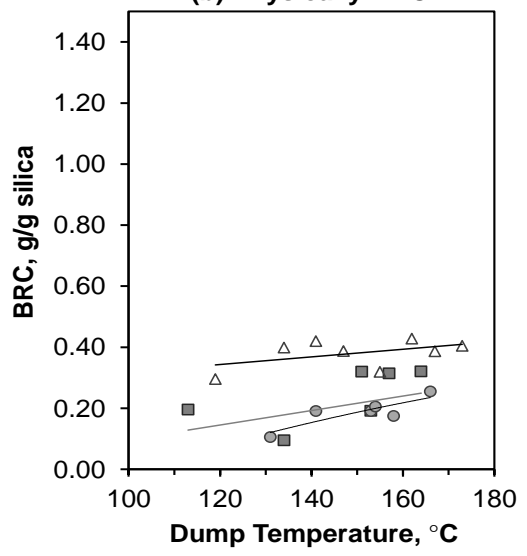

Figure 3.10 Comparison of: (a) chemically and (b) physically bound rubber content of silica

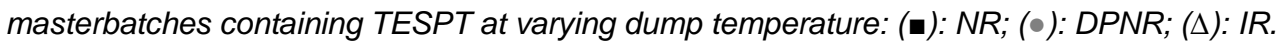

In Figure 3.10(b), the small increase in the physically bound rubber of the NR-silica and DPNR-silica compounds containing TESPT at higher dump temperature can be explained by the saturation of silica-TESPT coupling. Additional interactions above $150^{\circ} \mathrm{C}$ between the non-hydrophobized silica surfaces and rubber are physical of nature. The IR 68 
compound shows a more constant physically bound rubber which is comparable to that of NR: it is suggested as a result of physical adsorption and also the occluded rubber in silicasilica network for IR. As discussed above in the Payne effect, the in-rubber structure of IRsilica containing TESPT is comparable to those of NR and DPNR. The in-rubber structure includes the immobilization of rubber on the surface of silica due to silane modification, whether it is chemical or physical of nature and the occluded rubber in the silica network. With ammonia treatment, the immobilization of physically bound rubber in the silica structure or occluded rubber is released since physical interactions like the hydrogen bonding are destroyed.

\subsubsection{Mechanical and dynamic properties}

The use of TESPT as a coupling agent improves the stress-strain properties of silica-filled compounds. In Figure 3.11, it can be seen that NR-, DPNR- and IR-vulcanizates without silane exhibit inferior stress-strain behavior than those with silane. In comparison, the DPNR vulcanizates perform better than NR and IR in the presence and absence of coupling agent.

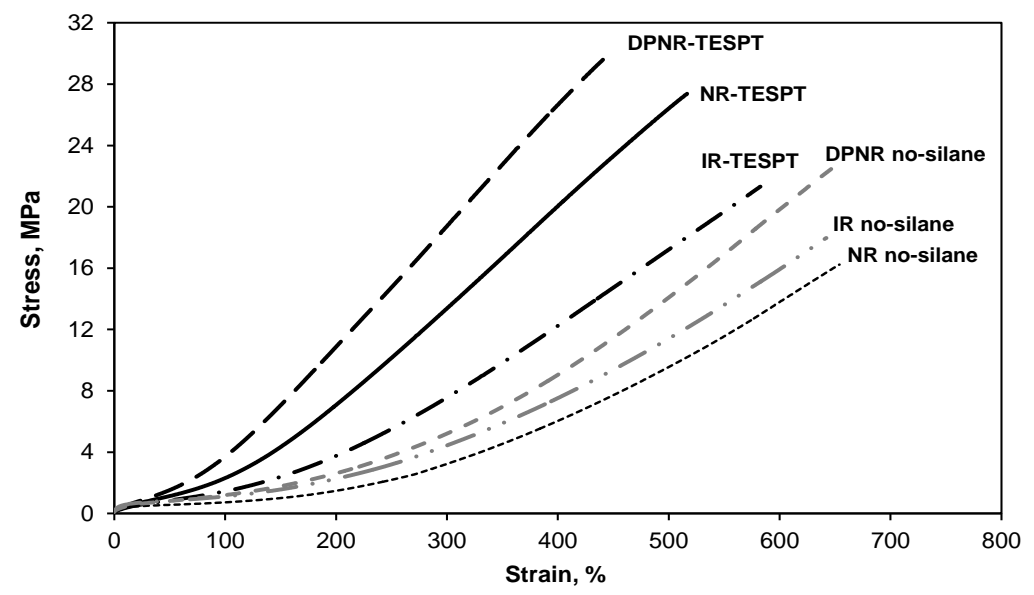

Figure 3.11 Comparison of stress-strain curves of NR-, DPNR- and IR-silica compounds with and without silane TESPT.

The effect of dump temperature on the physical properties of silica vulcanizates is depicted in Figure 3.12. At dump temperatures above $150^{\circ} \mathrm{C}$, both NR and IR vulcanizates show a clear reduction in tensile strength and the effect is most pronounced in the case of IR. This shows that mixing temperature is of importance for the NR-silica compounds. However, this reduction in tensile strength at high dump temperature is not seen for the low 
protein DPNR-silica vulcanizates. For the IR vulcanizate, the tensile strength is inferior to NR and DPNR vulcanizates. The elongation at break for the NR and DPNR vulcanizates reduces slightly with increasing dump temperature. The IR vulcanizates show a higher elongation at break as compared to NR and DPNR but are observed to decrease with mixing dump temperature as well.
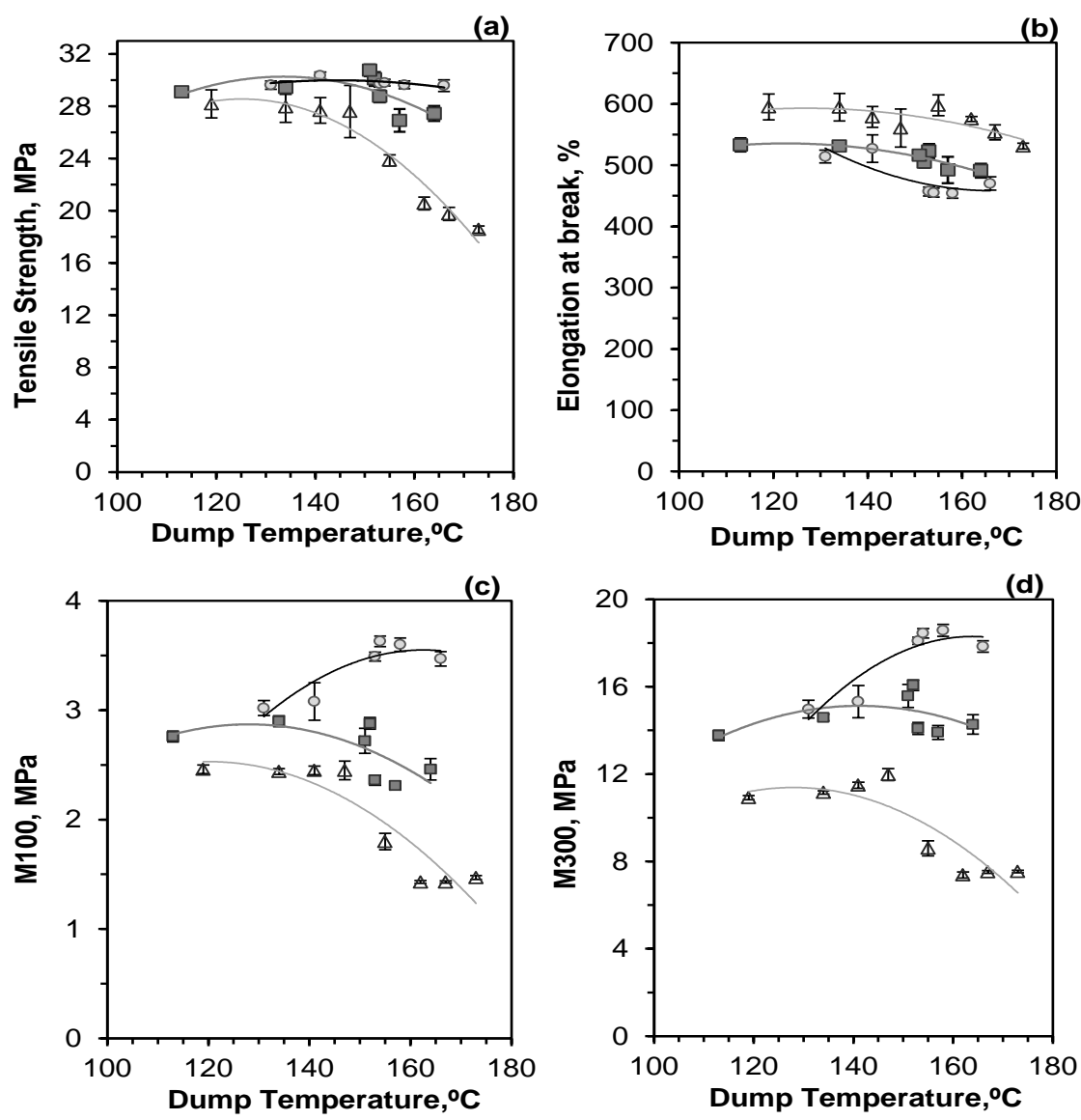

Figure 3.12 Physical properties of silica vulcanizates: (๓): NR; (๑): DPNR; $(\Delta)$ : IR;

(a) Tensile strength, (b) Elongation at break, (c) Modulus at 100\% elongation (M100), (d) Modulus at $300 \%$ elongation (M300).

A decrease in both moduli at 300\% elongation (M300) and 100\% elongation (M100) at higher dump temperatures is seen for the NR and IR vulcanizates. In contrast, DPNR vulcanizates exhibit higher moduli with the increasing mixing dump temperature. The 
reduction in the tensile properties of $N R$ and IR at high mixing temperature is in good agreement with the occurrence of reversion and decrease in the maximum torque observed in their vulcanization curves. In contrast, reversion is not seen in the vulcanization curve of DPNR and the effect of dump temperature on torque difference is also smaller. Another possible reason for the better properties achieved for DPNR is the difference in the naturally occurring networking as compared to NR. Amnuaypornsri et al. ${ }^{25}$ have proposed that the network structure of purified DPNR is associated with phospholipids linking both terminal end groups of the rubber chain via hydrogen bonding and ionic linkages, while the proteins bonds are released because of deproteinization.

Both, NR-silica and DPNR-silica, have a superior reinforcement index M300/M100 as compared to IR-silica vulcanizates as shown in Figure 3.13. With increasing mixing temperature, the reinforcement index of the NR and IR vulcanizates is somewhat improved. The processing conditions have less influence on the reinforcement index of DPNR. A better reinforcement index is obtained with higher chemically bound rubber of the compound as illustrated in Figure 3.13(b). It indicates that the improvement in properties is related to the increase in rubber to filler interaction.

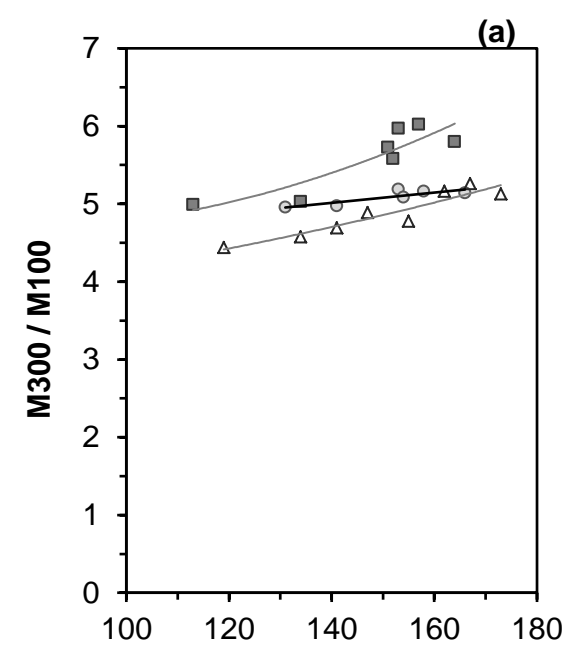

Dump Temperature, $\stackrel{\circ}{ } \mathrm{C}$

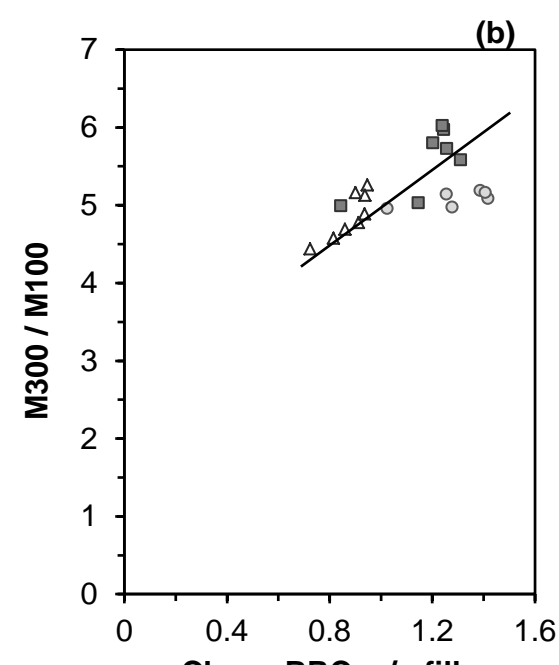

Chem. BRC, g/g filler

Figure 3.13 Reinforcement index M300/M100 of NR-, DPNR-and IR-silica compounds with TESPT as function of: (a) dump temperature; (b) chemically bound rubber; (घ):NR;

$$
\text { (०):DPNR; ( } \Delta) \text { : IR. }
$$


Commonly, the loss $\tan \delta$ at $60^{\circ} \mathrm{C}$ of a cured compound is employed as indication for the rolling resistance of tires made thereof. The lower the tan $\delta$ at $60^{\circ} \mathrm{C}$, the lower the rolling resistance expected in real tire performance. Figure 3.14 illustrates indications of rolling resistance of the silica-filled vulcanizates. Both NR and DPNR vulcanizates show a strong decrease in $\tan \delta$ at $60^{\circ} \mathrm{C}$ with increasing dump temperature. This must obviously be the result of more coupling of silica to the rubber with greater silanization efficiency at high temperatures. IR also shows a reduction in $\tan \delta$ at $60^{\circ} \mathrm{C}$ with increasing dump temperature, but only up to $150^{\circ} \mathrm{C}$. Above a dump temperature of $150^{\circ} \mathrm{C}$, the $\tan \delta$ at $60^{\circ} \mathrm{C}$ of the IR vulcanizates shows a marked increase. The DPNR vulcanizates exhibit the lowest $\tan \delta$ at $60^{\circ} \mathrm{C}$ at high dump temperature. This actually relates well with the higher chemically bound rubber content of DPNR compared to the NR compound.

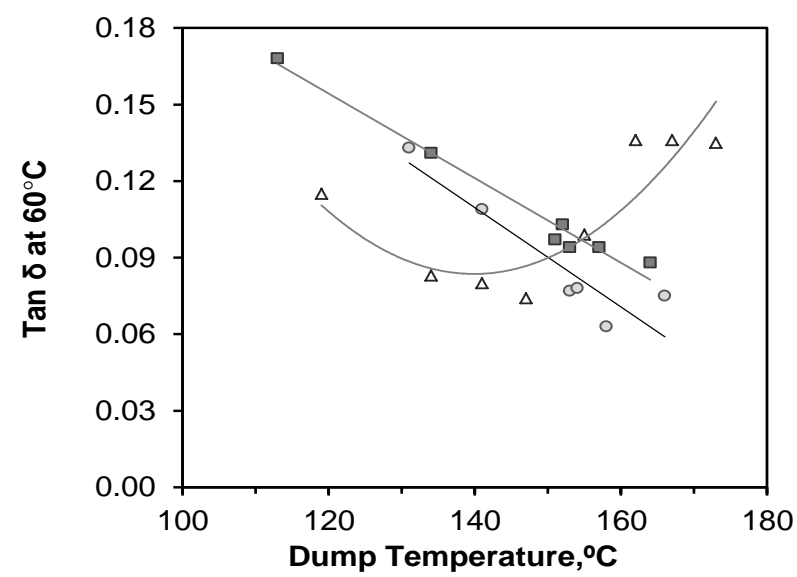

Figure 3.14 The effect of dump temperature on tan $\delta$ at $60^{\circ} \mathrm{C}$ of silica compounds: (匹):NR; $(\bullet): D P N R ;(\Delta): I R$.

In general, DPNR shows better mechanical and dynamic properties as compared to NR. In contrast, IR performs overall much worse, particularly if mixed till high dump temperature. The decrease in the mechanical properties of NR and IR vulcanizates mixed at high dump temperature correlates well with the decrease in the crosslink density.

This chapter illustrates in a phenomenological manner the reinforcement of various polyisoprenes by the silica-silane system. The results show the complicated nature of NR and that its influence on the mechanism of reinforcement is still unsatisfactorily understood. In addition to the chemical bonds between silica and rubber, the reinforcement 
of NR by silica fillers is also strongly governed by the polymer properties itself. NR is a unique natural product, subject to seasonal variations, and containing non-rubber constituents, such as proteins and phospholipids, and this makes this material very complex. The absence of these non-rubber constituents, in the case of IR, and removal of proteins, in the case of DPNR, show completely different behavior compared to NR even though they have nearly the same polyisoprene microstructure: till the last few percents poly(cis1,4-isoprene). How far the proteins and phospholipids affect the reinforcement of NR still needs further elucidation. Reinforcement of NR with silica remains a challenge but it offers tremendous benefit of utilizing renewable resources.

\subsection{CONCLUSIONS}

The key element in the reinforcement of rubber by silica is the chemical coupling between silica and rubber, which applies for both synthetic and natural rubbers. The investigation has demonstrated that the temperature development is an important parameter in mixing silica and natural and synthetic poly (cis 1,4-isoprene) with the aid of TESPT as coupling agent in order to achieve proper silanization of silica. Silica-silica interaction is reduced with hydrophobation of the silica surface by TESPT through silanization at sufficiently high mixing temperatures. In NR compounds, the silanization reaction between silica and TESPT progresses with increasing mixing dump temperature, which can be monitored with the drop in Payne effect of the compounds. Above an optimum dump temperature of approximately $150^{\circ} \mathrm{C}$, NR suffers some loss of dynamic and mechanical properties probably due to thermomechanical degradation. This effect is even more pronounced in the case of its synthetic equivalent: IR. On the other hand, removal of proteins from NR, in this case DPNR, results in a smaller influence of dump temperature and more constant mechanical properties. Dynamic properties improve with the silica-silane-rubber coupling as evidenced by a lower $\tan \delta$ at $60^{\circ} \mathrm{C}$ obtained especially in DPNR-silica-TESPT compounds.

\subsection{REFERENCES}

1. W. Meon, A. Blume, H-D. Luginsland, and S. Uhrlandt, "Silica and Silane", in Rubber Compounding: Chemistry and Applications, B. Rodgers, ed., Marcel Dekker, New York (2004)

2. J.W.M. Noordermeer and W.K. Dierkes, "Silica-Filled Rubber Compounds", in Rubber Technologist's Handbook Vol.2, J. White, S.K. De and K. Naskar, eds., Smithers Rapra Technology, Shawbury, Shrewsbury, Shropshire, UK, (2008).

3. S.S. Sarkawi, W.K. Dierkes and J.W.M. Noordermeer, presented at EU-PEARLS 2010 
Meeting: The Future of Natural Rubber, Montpellier, France (2010).

4. A.B. Othman and C. Hepburn, Plast. Rub. Compos. Pro., 19, 185 (1993).

5. Y. Tanaka and L. Tarachiwin, Rubber Chem. Technol., 82, 283 (2009).

6. J. Sakdapipanich, J. Biosci. Bioeng., 103, 287 (2007).

7. S. Amnuaypornsri, J. Sakdapipanich, S. Toki, B.S. Hsiao, N. Ichikawa and Y. Tanaka, Rubber Chem. Technol., 81, 753 (2008).

8. L. Tarachiwin, J. Sakdapipanich and Y. Tanaka, Kautsch. Gummi Kunstst., 58, 115 (2005).

9. B. Kastein and K.C. Baranwal, Chapter 9 in "Basic Elastomer Technology", Eds. K.C.

Baranwal and H.L. Stephens, Rubber Division ACS, Akron (2001).

10. E.C. Gregg and J.H. Macey, Rubber Chem. Technol., 46, 47 (1973).

11. Y. Tanaka, Rubber Chem. Technol., 74, 355 (2001).

12. S. Kawahara, T. Kakubo, N. Nishiyama, Y. Tanaka, Y. Isono and J. Sakdapipanich, J. Appl. Polym. Sci. 78, 1510 (2000).

13. S. Kawahara, Y. Isono, T. Kakubo, Y. Tanaka, and A.H. Eng, Rubber Chem. Technol., 73, 39 (2000).

14. S. Toki, J. Che, L. Rong, B.S. Hsiao, A. Nimpaiboon and J. Sakdapipanich, Fall 180th Technical Meeting, ACS Rubber Division, Cleveland, USA, paper no.9 (2011).

15. L.A.E.M. Reuvekamp, J.W. ten Brinke, P.J. van Swaaij and J.W.M. Noordermeer, Rubber Chem. Technol., 75, 187 (2002).

16. W.K. Kaewsakul, K. Sahakaro, W.K. Dierkes and J.W.M. Noordermeer, Rubber Chem. Technol., 85, 277 (2012).

17. S. Wolff, Rubber Chem. Technol., 55, 967 (1982).

18. M.J.R. Loadman and W.C. Wake, "Analysis of Rubber and Rubber-like Polymers", Kluwer Academic Publishers, the Netherlands, 96 (1988).

19. S. Wolff, Kautsch. Gummi Kunstst. 23, 7(1970).

20. K.E. Polmanteer and C.W. Lentz, Rubber Chem. Technol.,. 48, 795 (1975).

21. S. Wolff, M.-J. Wang and E.-H. Tan, Rubber Chem. Technol., 66, 163 (1992).

22. C.J. Lin, W.L. Hergenrother, E. Alexanian and G.G.A. Böhm, Rubber Chem. Technol., 75, 865 (2002).

23. S. Mihara, R.N. Datta and J.W.M. Noordermeer, Rubber Chem. Technol., 82, 524 (2009).

24. H.-D. Luginsland, J. Fröhlich and A. Wehmeier, Rubber Chem. Technol.,. 75, 563 (2002).

25. S. Amnuaypornsri, L. Tarachiwan and J. Sakdapipanich, J. App. Polym. Sci. 115, 3645 (2010). 
Chapter 4

\section{The Influence of Non-Rubber Constituents on Silica Reinforced Natural Rubber}

An in-rubber study of the interaction of silica with proteins present in natural rubber show that the latter compete with the silane coupling agent during the silanization reaction; the presence of proteins makes the silane less efficient for improving dispersion and fillerpolymer coupling, and thus influences the final properties of the rubber negatively. Furthermore, the protein content influences the rheological properties as well as filler-filler and filler-polymer interactions. Stress strain properties also vary with protein content, as do dynamic properties. With high amounts of proteins present in NR, the interactions between proteins and silica are able to disrupt the silica-silica network and improve silica dispersion. High amounts of proteins reduce the thermal sensitivity of the filler-polymer network formation. The effect of proteins is most pronounced when no silane is used, but they are not able to replace a coupling agent. ${ }^{a}$

\footnotetext{
${ }^{a}$ Parts of this chapter are published in Tire Technology 2012 p.18-22, Rubber World 247(2) p.26-31 (2012), KGK 66(3) p.27-33 (2013) and European Polymer Journal 49 p.3199-3209 (2013).
} 


\subsection{INTRODUCTION}

In recent years, the increasing demand for low-energy consuming and low rolling resistance tires has lead to growing use of silica in tread compounds. Four essential elements in silicarubber technology: the rubber polymer, a special type of silica, an effective coupling agent and the appropriate mixing technology are interconnected in expanding the magic triangle of tire technology: the compromise between rolling resistance, (wet) traction and wear. Compared to carbon black, mixing silica compounds involves many difficulties due to the large polarity difference between silica and rubber. A bifunctional organosilane such as bis(triethoxysilylpropyl) tetrasulfide (TESPT) or its disulfide equivalent is commonly used as coupling agent in enhancing the compatibility of silica and rubber, by chemically modifying silica surfaces and eventually creating a chemical link between silica aggregates and the rubber chains ${ }^{1}$. Complications arise during mixing silica compounds as several chemical reactions need to take place, all at their appropriate time slots during rubber processing, namely the silica and silane reaction or silanization, silane-rubber coupling and crosslinking between the rubber chains ${ }^{2}$.

The high-dispersion silica technology, as it is used today, employs mainly solutionpolymerised synthetic rubber, and is still not commercially feasible with natural rubber ${ }^{3}$. It was postulated that non-rubber constituents contained in natural rubber such as proteins compete with the coupling agent for reaction with the silica during mixing, so disturbing its reinforcement action ${ }^{3}$. However, no supporting evidence is available on this subject.

Commercial Natural Rubber (NR) comes from the milky sap or latex that exudes from the rubber tree, Hevea Brasiliensis, which coagulates on exposure to air. Hevea latex consists of rubber hydrocarbon for about 30-45 weight \%, non-rubber constituents for about 3-5 weight \%, and the rest is water. The non-rubber constituents comprise of proteins, amino acids, carbohydrates, lipids, amines, nucleic acids, as well as other inorganic and mineral components ${ }^{4}$. The work by Tanaka and coworkers has revealed that the fundamental structure of a linear NR chain consists of a long sequence of $1000-3000$ cis1,4 isoprene units, with at the $\alpha$ - and $\omega$-chain ends specific other groups ${ }^{5,6}$. The $\alpha$-terminal is composed of mono- and diphospate groups linked with phospholipids by hydrogen or ionic bonds ${ }^{7}$. The $\omega$-terminal entails two trans- 1,4 isoprene units $^{8}$ and a modified dimethylallyl unit linked with functional groups, which is associated with proteins to form crosslinks via hydrogen bonding ${ }^{9-10}$. Both non-rubber constituents, i.e. proteins and phospholipids are presumed to be the origin of branching and gel formation in $N R^{11}$. These secondary structures play a significant role in the strain-induced crystallization of unvulcanized and vulcanized natural rubber ${ }^{12,13}$. 
The protein content of NR varies upon its source and methods of production. The nitrogen content of the NR is related to the protein level. It is generally accepted that the conversion factor from nitrogen content to protein content is $6.25^{4,14}$. Typical raw NR has a nitrogen content in the range of $0.3-0.6 \%$. Many attempts have been made to purify NR from the non-rubber constituents such as proteins. One of the most successful attempts is the 'Deproteinized Natural Rubber' (DPNR) which is characterized by its very low nitrogen, ash and volatile matter contents compared to the equivalent commercial NR. DPNR is produced via treatment of natural rubber latex with bioenzyme (proteinase), which hydrolyses the proteins present into water soluble form ${ }^{15}$. In addition, deproteinization of natural rubber is also achieved in the latex stage with urea in the presence of a surfactant, such as sodium dodecyl sulphate ${ }^{16,17}$. On the other hand, during concentrated latex production, the serum phase after centrifugation contains $5-10 \%$ of the total rubber, and many of the non-rubbers. This is coagulated with sulfuric acid to produce skim rubber with a low dirt content and light colour and that is relatively cheap. Skim rubber has a high protein content ${ }^{18}$, where the nitrogen content has values in the range of $1.5-2.5 \%$.

Gregg and Macey ${ }^{19}$ have demonstrated that the insoluble non-rubber constituents in NR account for the differences in properties between compounded NR and compounded synthetic polyisoprene. This non-rubber material is mostly proteins and responsible for the higher modulus, faster scorch time and higher tear strength of NR. The protein is postulated to act as a reinforcing filler at low concentration (3-4 wt.\%) and as a cure activator. Othman and Hepburn [4] have shown that the presence of proteins from B-, C-serum and proteolipids did not significantly affect the elastic modulus of rubber vulcanizate. However, the presence of its hydrolyzed constituents, amino acids, gave a marked increase in the modulus of vulcanizates, in particular alanine and arginine, the basic and neutral amino acids, respectively ${ }^{20}$.

In the present chapter, the influence of non-rubber constituents in NR, particularly proteins, on the properties of silica-filled NR compounds in the presence and absence of coupling agent is illustrated. In order to demonstrate the variation of proteins content in NR, DPNR and skim rubber are selected in comparison with normal NR. The filler-filler and fillerto rubber interactions of silica reinforced NR compounds at varying mixing dump temperatures are highlighted. 


\subsection{EXPERIMENTAL}

\subsubsection{Materials}

Natural rubbers with different protein contents were compared in this study. The rubbers with their protein contents based on nitrogen estimations are summarized in Table 4.1. It is generally accepted that the conversion factor from nitrogen content to protein content is $6.25^{4,14}$. The nitrogen contents of the three rubbers were determined by the semi-micro Kjeldahl procedure carried out by the Materials Characterization Unit, Malaysian Rubber Board (MRB). The compound formulation used throughout this investigation is shown in Table 4.2. Highly dispersible silica, Ultrasil 7005 from Evonik with CTAB surface area of 164 $\mathrm{m}^{2} / \mathrm{g}$ was used. The other compounding ingredients were used as obtained from the respective sources.

Table 4.1 Protein content of natural rubber's used

\begin{tabular}{lcc}
\hline \multicolumn{1}{c}{ Rubber Type } & Nitrogen content, wt\% & Protein content, wt\% \\
\hline NR (SMR 20) & 0.21 & 1.3 \\
DPNR (Pureprena) & 0.07 & 0.4 \\
Skim Rubber & 2.06 & 12.9 \\
\hline
\end{tabular}

Table 4.2 Compound Formulation

\begin{tabular}{lll}
\hline Ingredients & Source & phr \\
\hline Natural Rubber (various types) & MRB & $100^{\mathrm{a}}$ \\
Silica Ultrasil 7005 & Evonik & 55 \\
TESPT & Evonik & $5^{\mathrm{b}}$ \\
Zinc Oxide & Sigma Aldrich & 2.5 \\
Stearic acid & Sigma Aldrich & 1 \\
TDAE oil & Hansen \& Rosenthal & 8 \\
2,2,4-trimethyl-1,2-dihydroquinoline (TMQ) & Flexsys B.V. & 2 \\
Sulfur & Sigma Aldrich & 1.4 \\
N-cyclohexyl-2-benzothiazyl sulphenamide (CBS) & Flexsys B.V. & 1.7 \\
Diphenyl guanidine (DPG) & Flexsys B.V. & 2 \\
\hline${ }^{a}$ For skim rubber, the formulation was adjusted to 112 phr to take into account the high protein content; \\
${ }^{b}$ For compound without silane, TESPT is omitted from the formulation
\end{tabular}

\subsubsection{Sample Preparation}

The procedures and equipments used for the compound mixing process were as previously described in the experimental section in Chapter 3. 


\subsubsection{Sample Analyses}

Mooney viscosity, vulcanization curves, Payne effect, Wolff's filler structure parameter $\alpha_{f}$, bound rubber content, tensile properties, hardness and tan delta at $60^{\circ} \mathrm{C}$ of the compounds were investigated according to the methods as described in the experimental section in Chapter 3.

An apparent crosslink density was determined by swelling a vulcanized sample in toluene. The vulcanized sample of about $0.2 \mathrm{~g}$ was cut from a sheet with a thickness of $2 \mathrm{~mm}$ and immersed in $50 \mathrm{ml}$ toluene at room temperature for 72 hours. The solvent was renewed after 24 hours. The sample was removed, blotted quickly with filter paper and weighed in a tared weighing bottle. The samples were immersed in acetone for 30 minutes to remove the remaining solvent. The samples were collected and left for 24 hours at room temperature in a fume hood before the dried weight was measured. The swelling value $Q$, defined as grams of toluene per gram of rubber hydrocarbon, was calculated as:

$$
Q=\left(\frac{\text { swollen weight-dried weight }}{\text { sample weight }}\right) \times\left(\frac{\text { formula weight }}{100}\right)
$$

where formula weight is the total weight of all compounding ingredients including rubber based on 100 parts of rubber (phr). The apparent crosslink density was calculated as the reciprocal swelling value, $1 / Q$.

\subsection{ResULtS AND Discussion}

\subsubsection{Processability of silica-filled NR compounds}

In terms of processability of the masterbatches, NR and DPNR are comparable, but skim rubber has a lower viscosity. In Figure 4.1, the increase in viscosity of the masterbatches with rising mixer dump temperature up to a temperature of $150^{\circ} \mathrm{C}$ is a combination of the hydrodynamic effect and silanization rate of the silica. More silica is hydrophobized by TESPT when the dump temperature is raised, and this results in a higher compatibility between silica and rubber and consequently increment of the viscosity. However, the viscosity of the masterbatches of NR and DPNR start to decrease above the optimum dump temperature, but in the case of skim rubber it levels off. One explanation is the degradation of the NR chains at higher temperatures, which seems to be inhibited by a high protein content.

Once the curatives are added to the compounds, the viscosities drop to processable levels, mainly due to the remilling step. In spite of the overall lower Mooney viscosities of the skim rubber masterbatches after the first mixing step, the Mooney 
viscosities of the compounds with curatives after mill mixing are almost comparable with those of the NR and DPNR compounds. A comparable Mooney viscosity between NR, DPNR and skim rubber compounds is due to many factors which include silica dispersion, the interaction of polar curatives with silica, the remilling step and reduction of chain entanglements or pseudo network of rubber chains.

(a) masterbatches

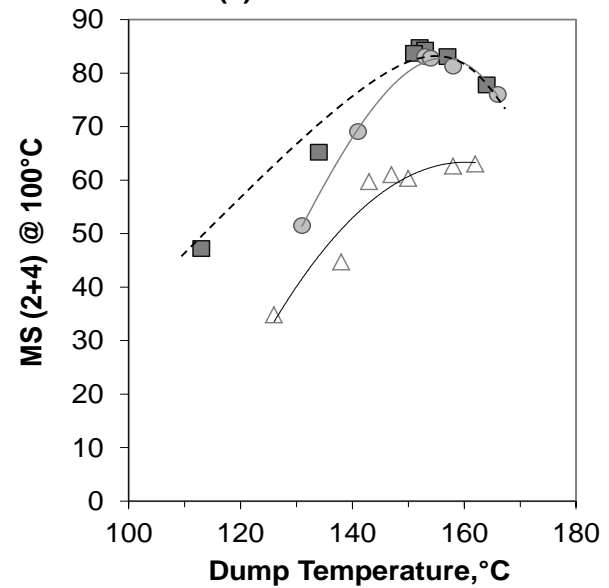

(b) compounds

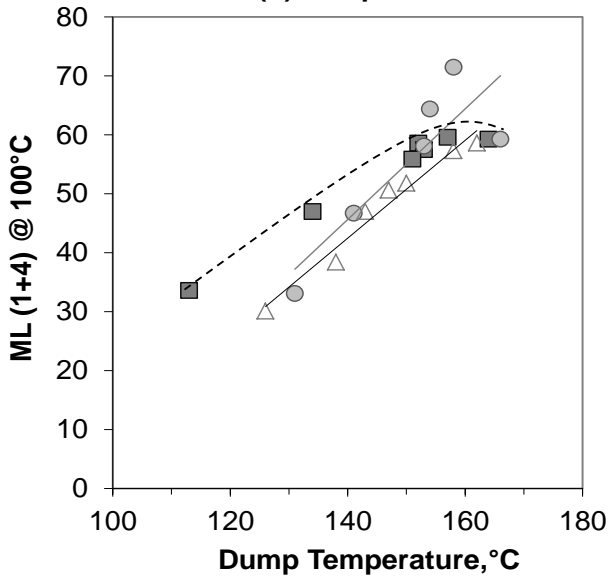

Figure 4.1 Mooney viscosities of (a) masterbatches after $1^{\text {st }}$ mixing, and (b) compounds after $2^{\text {nd }}$ mill mixing of silica-filled natural rubber at varying protein contents: $(\bullet): 0.4 \%$ (DPNR); (ロ): 1\% (NR); ( $\Delta): 12 \%$ (SkimRubber).

\subsubsection{Effect of protein on filler-filler interaction}

The influence of proteins in NR on the silica-silica interaction can be clearly observed from the vulcanization curve as depicted in Figure 4.2. The clear two-step curve for NR-silica and DPNR-silica compounds without silane is due to the silica flocculation ${ }^{21,22}$ or reagglomeration and strong silica networking. With high amounts of protein present in the compound, the silica-silica interaction is disrupted and this is shown with no sign of flocculation at the beginning of vulcanization for the skim rubber-silica compound without silane. A longer scorch time and slightly faster cure time is also observed for the skim rubber compound indicating an effect of protein on vulcanization speed as well.

The use of a silane, TESPT in this case, in the NR-silica compound results in less pronounced silica flocculation and this is demonstrated by a small initial torque rise at the beginning of vulcanization (Figure 4.2). As compared to the silica compounds without silane, the flocculation of silica in the compounds with TESPT is small due to hydrophobization of 
the silica surface by TESPT. The effect of protein on the cure behavior of the silica compounds fades with the presence of TESPT.
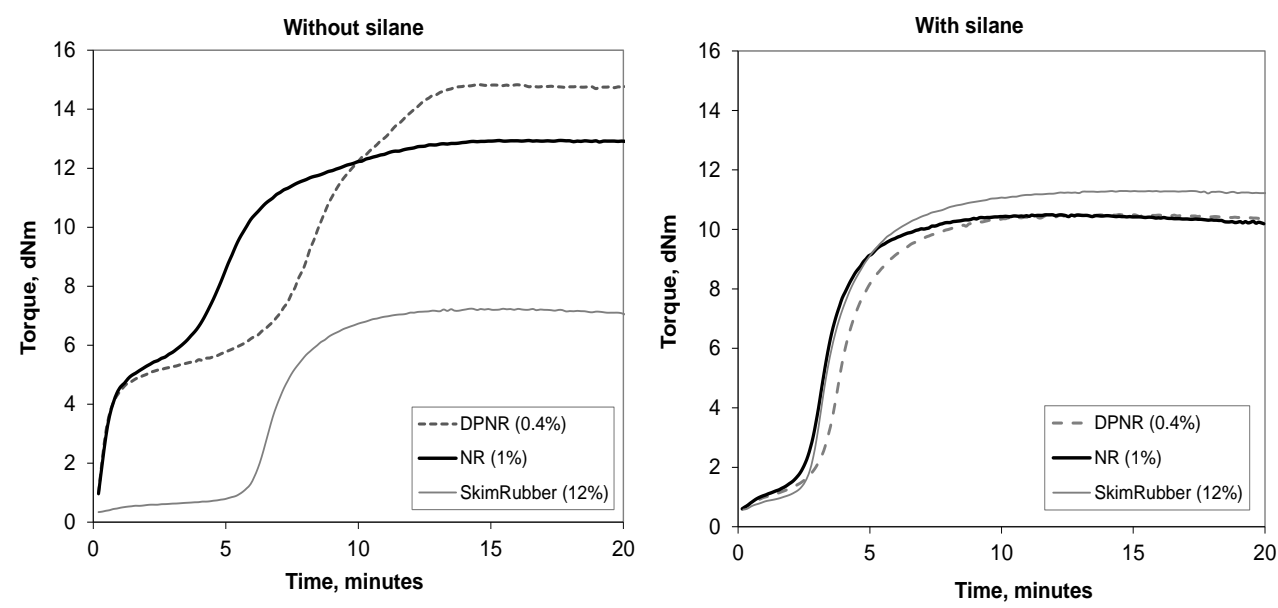

Figure 4.2 Comparison of vulcanization curves of silica compounds with silane and without silane.

The thermal history and in particular the dump temperature has been shown to be a parameter of paramount importance in mixing silica and rubber in presence of TESPT as coupling agent in order to achieve proper silanization of silica and to avoid premature scorch reactions ${ }^{23,24}$. The strong influence of dump temperature on the NR-silica-TESPT compound is reflected in the torque difference ( $\Delta$ torque) and cure rate index as shown in Figure 4.3. At high dump temperature, the NR-silica-TESPT compound exhibits a considerable reduction in $\Delta$ torque and a sudden remarkable increase in the cure rate index. It seems that with increasing silanization, the NR compound shows a shorter cure time due to enhancement in cure efficiency with the use of TESPT. This leads to anomalous behavior of the cure rate index for the NR compound mixed at higher temperature. Moreover, with higher silanization filler-filler interactions are reduced and this leads to lower maximum torque. DPNR-silicaTESPT also shows a decrease in $\Delta$ torque with increasing dump temperature, but to a lesser extent than that of NR. On the other hand, the high protein content skim rubber-silicaTESPT compound shows a different behavior where $\Delta$ torque is not influenced by the dump temperature. This may indicate a strong influence of the proteins in the silica compound and suggests the contribution of a silica-protein network in the cured compounds.

Filler-filler interaction is commonly measured by the so-called Payne effect: the drop in storage modulus in a dynamic mechanical test when the strain (deformation) is 
increased from low $(0.56 \%)$ to a high value $(100 \%)$ at constant frequency and temperature. The storage modulus of filled rubber drastically decreases as the strain increases. This is the result of breakage of physical bonds between filler aggregates, for example van der Waals, hydrogen bonds and London forces.
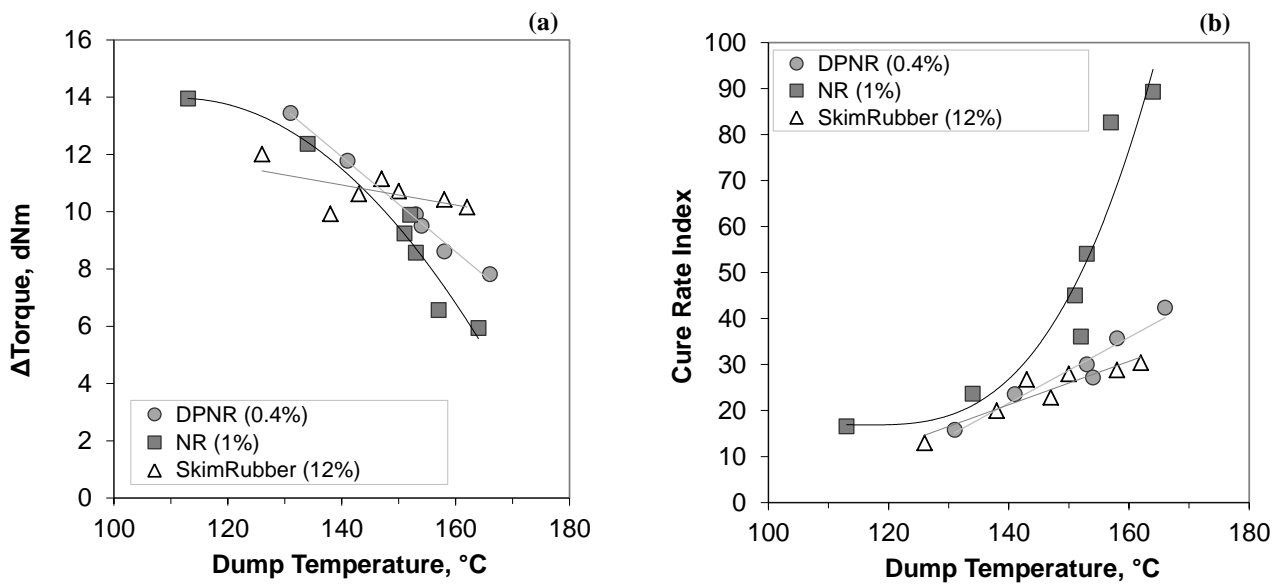

Figure 4.3 Comparison of the cure characteristic of NR-silica-TESPT compounds with different protein contents as influenced by mixing dump temperature: (a): Torque difference; (b): Cure rate index.

The use of silica without silane modification in rubber results in a high Payne effect due to strong interaggregate interaction of silica. With TESPT modification, the Payne effect of the silica-filled compounds is greatly reduced as the silica surface is hydrophobized by TESPT, and the silica-silica network is disrupted as shown in Figure 4.4. What is interesting in this study is that the same effect can be seen with protein. With a high amount of protein present in the rubber, the Payne effect of the silica compound without silane is lowered. There is a relation between the amount of protein and the decrease of silica-silica interaction. This indicates a strong affinity of the proteins towards silica, as well as the role of proteins in hydrophobizing the silica surface. The interaction between proteins and silica most likely come from hydrogen bonding between silanol groups on the surface of the silica to amide and carboxyl groups of proteins. In the presence of a silane, the effect of protein comes more pronounced in the Payne effect after vulcanization. An increase in the Payne effect at higher protein content indirectly indicates competition between protein and silane for the silica surface. This results in reduction of silanization efficiency and consequently less hydrophobation of the silica surface. 
(a) Before vulcanization

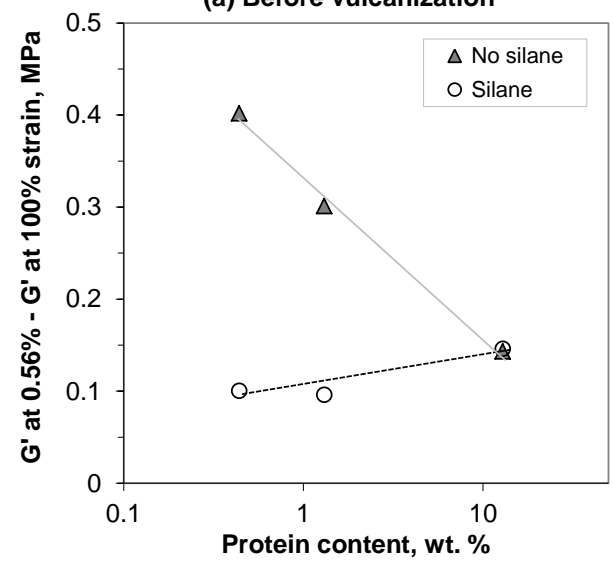

(b) After vulcanization

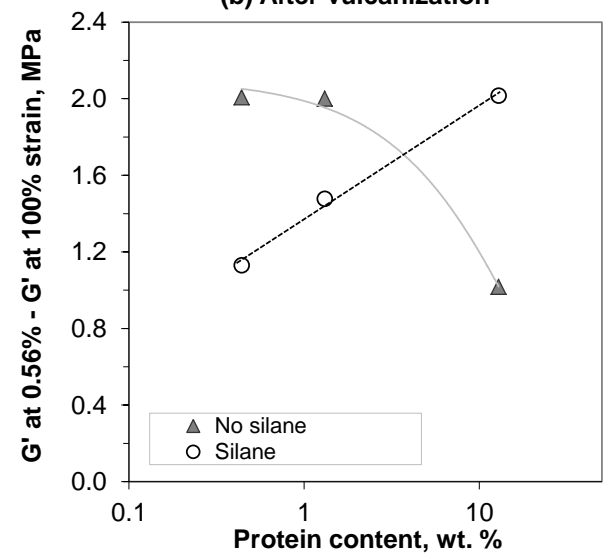

Figure 4.4 Influence of proteins on the Payne effect of NR-silica compounds: (a) Before vulcanization (b) After vulcanization ; (०): Silica compounds with silane TESPT ; (A): Silica compounds without silane.

(a) Before vulcanization

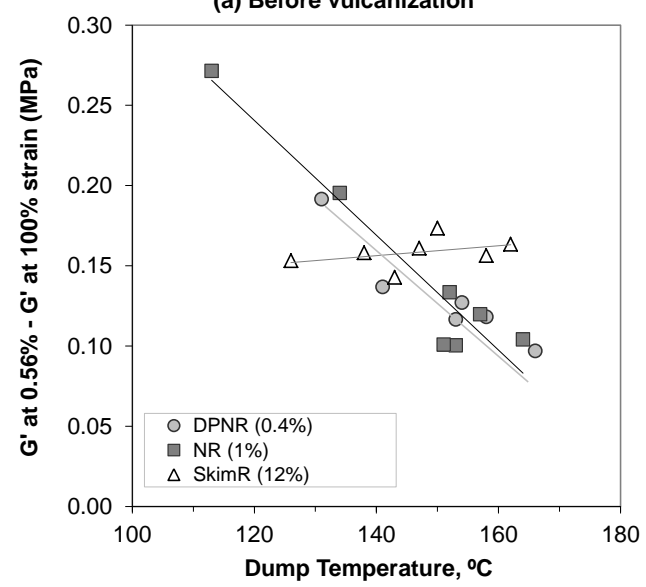

(b) After vulcanization

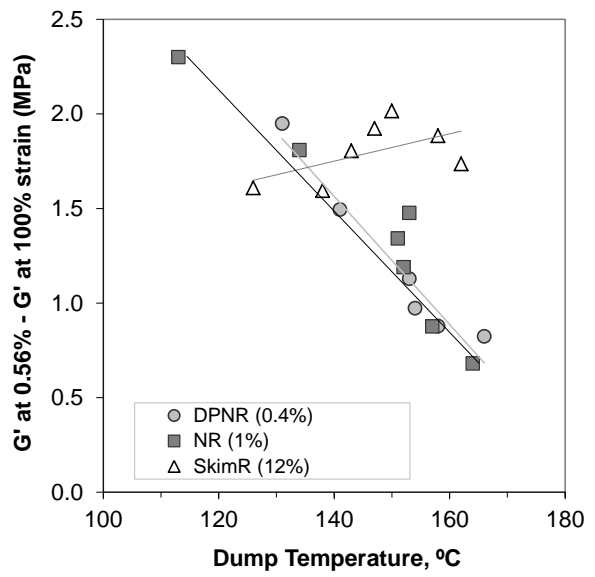

Figure 4.5 Payne effect of silica compounds with TESPT at varying protein contents in natural rubber: (a) unvulcanized samples; (b) vulcanized samples.

For silica compounds with silane, the Payne effect of NR and DPNR compounds decreases sharply with increasing dump temperature, as is also seen in synthetic rubber / silica compounds and taken as a sign of reaction and consequent hydrophobation of the silica by the silane coupling agent ${ }^{23,24}$. No effect of mixing temperature is perceived on fillerfiller interaction for the skim rubber compound, Figure 4.5; this is observed for the 
unvulcanized as well as for the vulcanized compounds. This again indicates a strong interference of the proteins in skim rubber with the filler-filler network. For skim rubber, the silica-silica network is not influenced by the dump temperature even in the presence of silane because silanization is hindered. It can be seen in Figure 4.5 that the Payne effect of the vulcanized skim rubber compound is higher than for the NR and DPNR compounds for the higher dump temperatures. The proteins in the skim rubber prevent the modification of the silica surface by the silane coupling agent. The logical explanation is that the interaction between silica and protein overrules the coupling agent and that the protein is shielding the silica surface.

\subsubsection{Effect of protein on rubber-to-filler interaction}

The difference between the NR compounds with varying protein content is further illustrated in Figure 4.6, covering the effect of mixing temperature on Wolff's filler structure parameter, $\alpha_{f}$. For compounds without silane, $\alpha_{f}$ is much higher than for those with TESPT, except for skim rubber. The $\alpha_{f}$ value for skim rubber without silane is even lower than the typical values obtained by Wolff for carbon black compounds: Table $4.4^{25}$. As observed earlier with the Payne effect, $\alpha_{f}$ is reduced with increasing dump temperature for NR and DPNR compounds containing silane. This is due to the increased hydrophobation of the silica by the silanization reaction at higher mixing temperatures. Better hydrophobation leads to a decrease in silica-silica interaction and consequently results in reduced $\alpha_{f}$. The $\alpha_{f}$ of NR mixed till high dump temperature is comparable to the typical value obtained by Wolff for TESPT-modified-silica compounds based on NR: Table 4.4. The DPNR compound shows a higher $\alpha_{f}$ than the NR compound, indicating a different type of filler and rubber network in the two compounds. For the high protein content skim rubber compound, the $\alpha_{f}$ value is much smaller than for the NR and DPNR compounds, and is constant regardless of the changes in the dump temperature. This corresponds with the results of its Payne effect described above. The non-rubber constituents or proteins in the skim rubber again plays the main role in the formation of the filler structure, and mixing temperature again has little influence on the compound properties.

Table 4.4 Typical Wolff's filler structure parameters $\left(\alpha_{f}\right)^{25}$

\begin{tabular}{lc}
\hline \multicolumn{1}{c}{ Filler } & $\boldsymbol{\alpha}_{\mathbf{f}}$ \\
\hline Carbon Black $^{\text {CoraxN110 }}$ & 1.86 \\
Unmodified Silica $^{\text {Ultrası VN2 }}$ & 5.65 \\
TESPT treated Silica & 1.84 \\
\hline
\end{tabular}




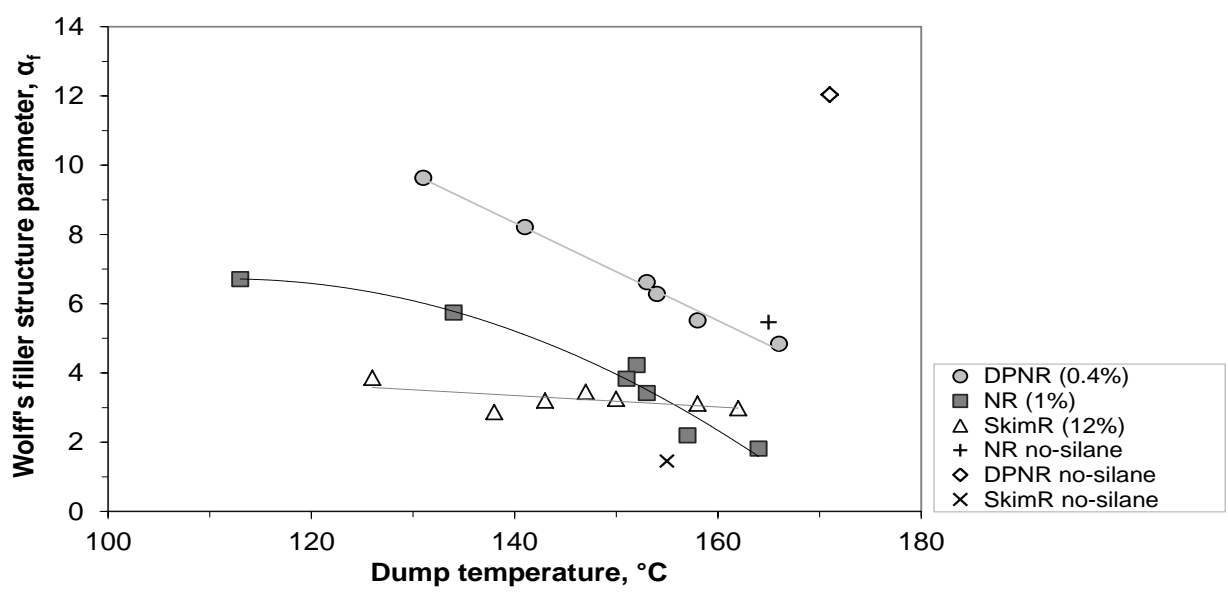

Figure 4.6 Effect of dump temperature on Wolff's filler structure parameter, $\alpha_{f}$, of silica reinforced natural rubber with varying protein contents.

The filler to rubber interaction of silica-filled NR with varying protein content can also be judged on basis of the chemically and physically bound rubber as illustrated in Figure 4.7. The chemically bound rubber content of the NR-silica-TESPT compound increases with increasing dump temperature up to $150^{\circ} \mathrm{C}$, and above $150^{\circ} \mathrm{C}$ it tends to decrease slightly. This can be explained by the higher rate of silanization. At $150^{\circ} \mathrm{C}$, there is saturation in the amount of TESPT which has reacted and the surface of silica is completely covered. Hence, there is no further increase in chemically bound rubber above $150^{\circ} \mathrm{C}$. The low protein DPNR compound also shows an increase in chemically bound rubber with increasing dump temperature, but has an optimum at $155^{\circ} \mathrm{C}$ dump temperature. The skim rubber compound has no chemically bound rubber at all at low dump temperatures below $140 \stackrel{\circ}{\circ}$. This clearly indicates that no silanization occurs and that the interaction of protein and silica in skim rubber is purely physical of nature. Surprisingly, above $150^{\circ} \mathrm{C}$, the skim rubber compound has a constant, and high chemically bound rubber content comparable to DPNR. This suggests that some part of the silica surface is silanized although at lower efficiency due to competition between protein and silane as discussed in the filler-filler interactions. Another explanation of the higher chemically bound rubber of skim rubber is the effect of crosslinks generated by sulfur released from TESPT at the high mixing temperature. This premature crosslinking is possibly accelerated by protein in the case of skim rubber.

In Figure 4.7(b), the increase in physically bound rubber for the NR-silica compound at higher dump temperatures can be explained by the completion of the silica- 
TESPT coupling. Additional interactions above $150^{\circ} \mathrm{C}$ between the non-hydrophobized silica surfaces and rubber are physical of nature. For the low protein content DPNR compound, the physically bound rubber is lowest throughout the whole dump temperature range, but also still shows a slight tendency to grow at higher dump temperatures. Further, a strong interaction between protein and silica can be seen in the high physically bound rubber values for the skim rubber at low dump temperatures. Above dump temperature of $150^{\circ} \mathrm{C}$, an equal level of physically bound rubber of skim rubber and DPNR is observed indicating the physical interactions caused by proteins are destroyed.

(a) Chemically BRC

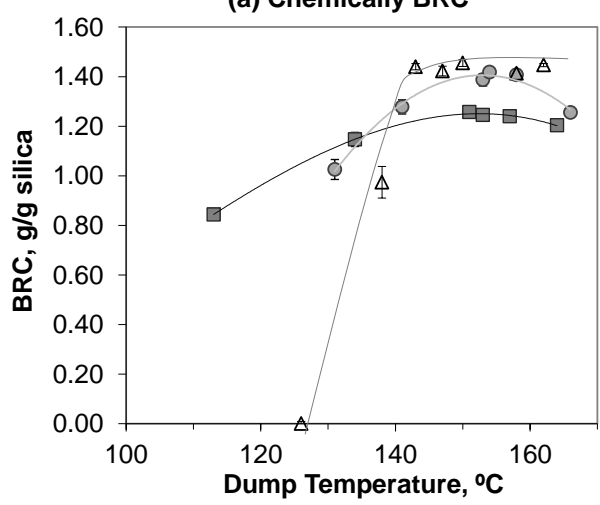

(b) Physically BRC

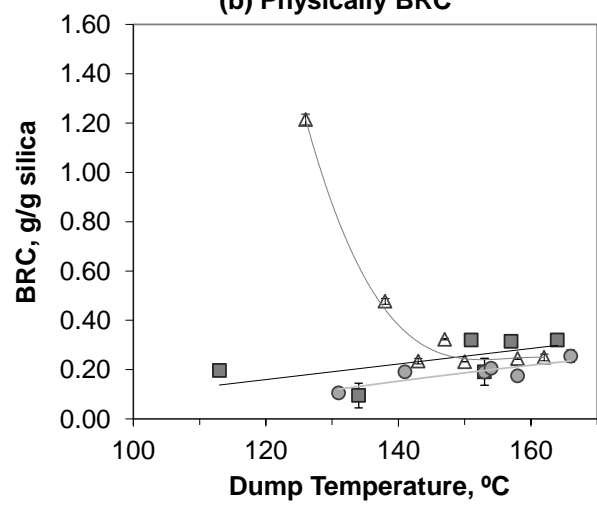

Figure 4.7 Comparison of (a) chemically and (b) physically bound rubber content of NRsilica-TESPT compounds with varying protein content: (•): 0.4\% (DPNR); (घ): $1 \%(N R) ;(\Delta)$ : 12\% (SkimRubber)

Most of the bound rubber formed in a NR-silica-TESPT compound is chemically attached. This is obviously due to the hydrophobation of the silica surface as a result of silanization with TESPT. The increase in silica-TESPT coupling consequently results in more filler-to-rubber interaction. This corresponds well with the lower Payne effect of the silica compounds with TESPT. The trends seen in chemically bound rubber correlate surprisingly well with the Mooney viscosities of the masterbatches with the exception of skim rubber: Figure 4.8. Apparently, when more chemically bound rubber is formed, the molecular motion of the rubber chains is restricted and this results in higher Mooney viscosity. This emphasizes the importance of chemical coupling of the rubber to the silica surface. In the skim rubber, the increase in the chemically bound rubber seems to be constrained, as the silanization is hindered by protein, and hence no changes in the Mooney viscosity are observed. 


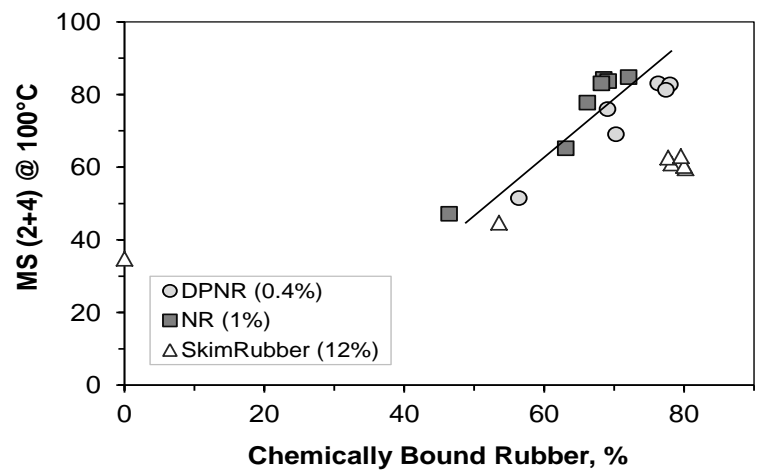

Figure 4.8 Mooney viscosity of NR-silica-TESPT masterbatches as a function of chemically bound rubber.

Table 4.5 Bound Rubber Content (BRC) of silica compounds without silane

\begin{tabular}{lcc}
\hline Rubber type & Physically BRC (\%) & Chemically BRC, \% \\
\hline NR & 57 & 0 \\
DPNR & 45 & 0 \\
Skim rubber & 51 & 0 \\
\hline
\end{tabular}

Without silane in the compounds, there is still silica-rubber interaction, as indicated by the physically bound rubber in Table 4.5. There are many factors that could contribute to the formation of bound rubber content, which involves physical adsorption, chemisorption and mechanical interaction. The results demonstrate that the proteins contained in skim rubber do interact with the silica, mainly via hydrogen bonding, make it less hydrophilic and thus increase the rubber-silica interaction. A comparison of filler-to-rubber interactions in compounds with silane and without silane is graphically represented in Figure 4.9. However, no chemically bound rubber was obtained for the silica compounds without silane after ammonia treatment because all the hydrogen bonds are destroyed. This means that without silane in the compound, only loosely or physically bound rubber is formed. This again indicates that the interaction of silica with NR in the absence of coupling agent is not as strong as compared to covalent bonds in a compound with silane. A slightly higher physically bound rubber content for the NR than for the skim rubber compound relates to the effect of higher molecular weight of NR. Many studies have established increase of bound rubber and preferential adsorption onto reinforcing fillers with higher molecular weight polymers ${ }^{26-28}$. A longer rubber chain will have more inter-aggregate connections, and this will contribute to more bound rubber formation. In addition, filler morphologies also a 
contributing factor to formation of bound rubber. The stronger silica-silica interaction in the NR compound without silane as shown by the higher Payne effect increases the bound rubber content. This is in accordance with the occluded bound rubber model where the rubber is trapped in the filler aggregate.

(a) Silica - Silane - NR

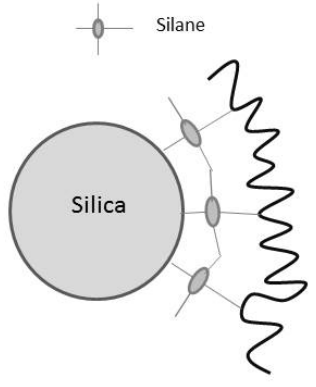

(b) Silica - Protein - NR

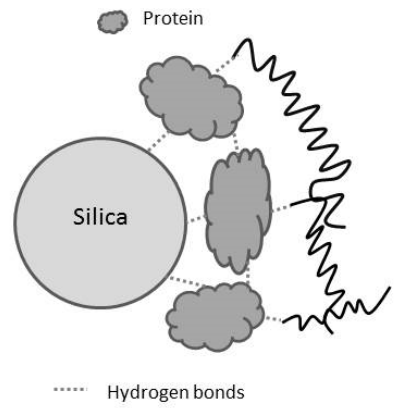

Figure 4.9 Illustration on the filler-to-rubber interaction for a silica compound: (a): with silane;(b): with protein and without silane.

\subsubsection{Physical and dynamic properties of silica reinforced NR}

The use of TESPT as a coupling agent improves the vulcanizate properties of silica-filled compounds. Vulcanizates without silane exhibit inferior tensile strength than those with silane as depicted in Figure 4.10. NR-silica-TESPT vulcanizates mixed till higher dump temperatures exhibit a slight drop in tensile strength, elongation at break and moduli values. On the other hand, the effect of dump temperature is less pronounced for DPNR-silicaTESPT vulcanizates, showing more constant tensile strength. This relates to the crosslink density of the vulcanizates as shown in Figure 4.11. The DPNR-silica-TESPT vulcanizates exhibit a rather constant crosslink density while NR-silica-TESPT vulcanizates show a reduction in crosslink density at higher dump temperature. The decrease in the elongation at break for NR and DPNR when the dump temperature exceeds $150^{\circ} \mathrm{C}$ is most probably caused by the degradation of natural rubber chains under high temperature. NR and DPNR vulcanizates have comparable hardness and show quite a decrease in hardness with increasing dump temperature. The skim rubber vulcanizates perform overall much worse compared to NR and DPNR vulcanizates in terms of physical properties, due to lack of silica-rubber coupling and lower molecular weight of the polymers to start with. Skim rubber shows higher modulus and hardness. This correlates with the highest crosslink density of skim rubber compared to NR and DPNR as shown in Figure 4.11. The higher crosslink of 
skim rubber is also in agreement with the earlier discussion on the premature crosslinks generated by sulfur released from TESPT during high mixing temperature, which results in higher chemically bound rubber. This premature crosslinking is possibly accelerated by proteins in the skim rubber.

(a)

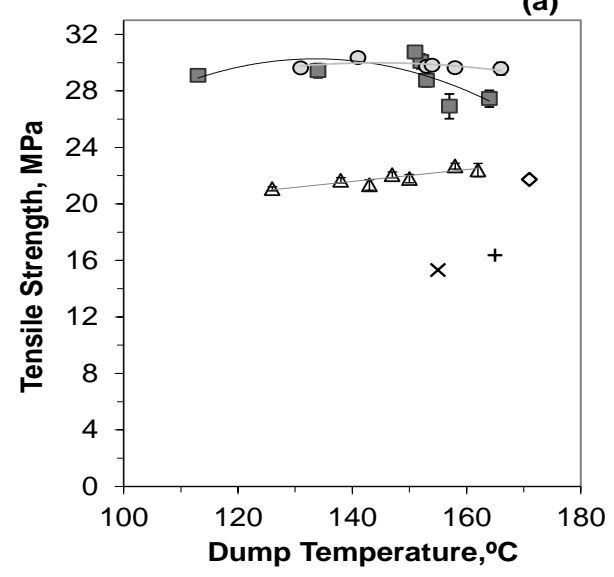

(c)

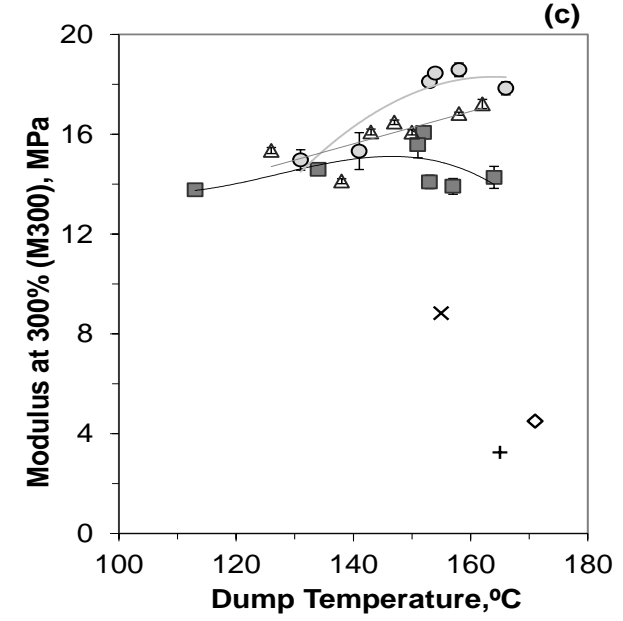

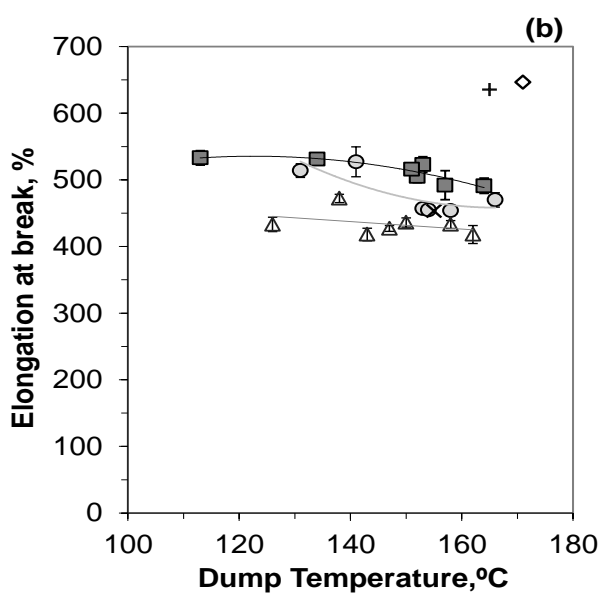

(d)

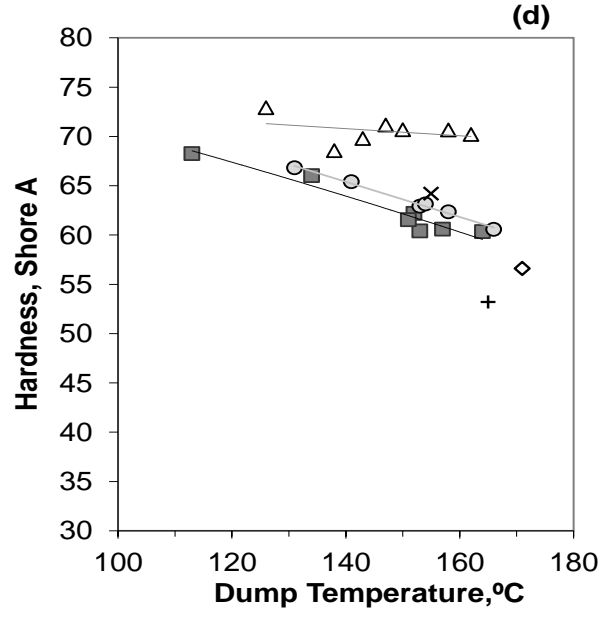

Figure 4.10 Comparison of physical properties of silica-TESPT-NR vulcanizates with different amounts of protein content: (•): 0.4\% (DPNR); (ロ): 1\% (NR); $(\Delta): 12 \%$ (SkimRubber); and silica compounds without silane: (+) NR; ( $)$ : DPNR; (x) Skim Rubber.

Commonly, two characteristic properties of cured compounds are accepted as indications for the rolling resistance of tires made thereof: the reinforcement index or $\mathrm{M} 300 / \mathrm{M} 100$ and the loss angle $\tan \delta$ at $60^{\circ} \mathrm{C}$. The higher the M300/M100 or the lower the 
$\tan \delta$ at $60^{\circ} \mathrm{C}$, the lower the rolling resistance expected in real tire performance. Figure 4.12 illustrates both indicators of rolling resistance of the silica-filled vulcanizates. Both, NR-silica and DPNR-silica have a superior reinforcement index as compared to skim rubber-silica vulcanizates. With increasing mixing temperature, the reinforcement index of the NR vulcanizate is somewhat improved. The processing conditions have no influence on the reinforcement index M300/M100 of DPNR and skim rubber vulcanizates, where skim rubber is substantially lower than NR and DPNR. These data seem to indicate that the correlation between reinforcement index and rolling resistance only applies for NR, but not for DPNR and skim rubber. This also relates to the constant crosslink density of DPNR and skim rubber vulcanizates.

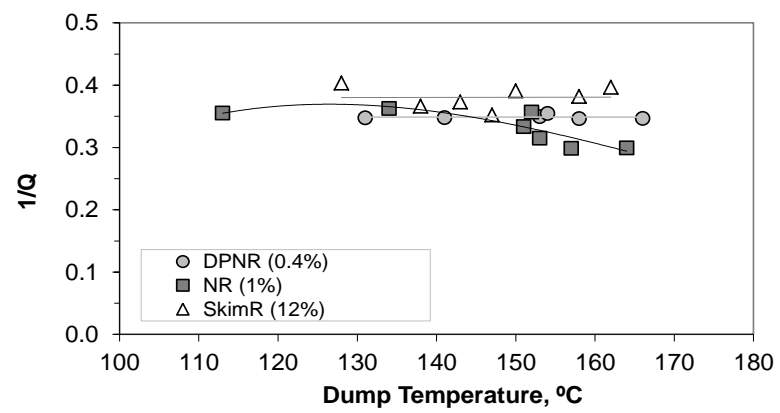

Figure 4.11 Apparent crosslink density of silica-TESPT reinforced natural rubber vulcanizates with varying protein content.

All natural rubber vulcanizates show a strong decrease in $\tan \delta$ at $60^{\circ} \mathrm{C}$ with increasing dump temperature regardless of the amount of protein in the rubber. Improvement in $\tan \delta$ at $60^{\circ} \mathrm{C}$ can still be achieved with higher mixing temperatures, like with synthetic rubber. This must obviously be the result of more coupling of silica to the rubber with greater silanization efficiency at high temperatures. This is further supported by the high $\tan \delta$ at $60^{\circ} \mathrm{C}$ for silica compounds without silane. Due to low protein content, the DPNR vulcanizates with high dump temperatures exhibit the lowest $\tan \delta$ values at $60^{\circ} \mathrm{C}$. This actually relates well with a higher chemically bound rubber content of DPNR than of NR. Still with all the protein contained in skim rubber, the $\tan \delta$ at $60^{\circ} \mathrm{C}$ is significantly lowered by increasing mixing temperature, and only marginally worse than for NR and DPNR. It is further quite surprising that the reinforcement index and tan $\delta$ at $60^{\circ} \mathrm{C}$ show opposite behavior in the present NR systems, compared to synthetic rubber based silica-filled compounds $^{29}$. 
(a)

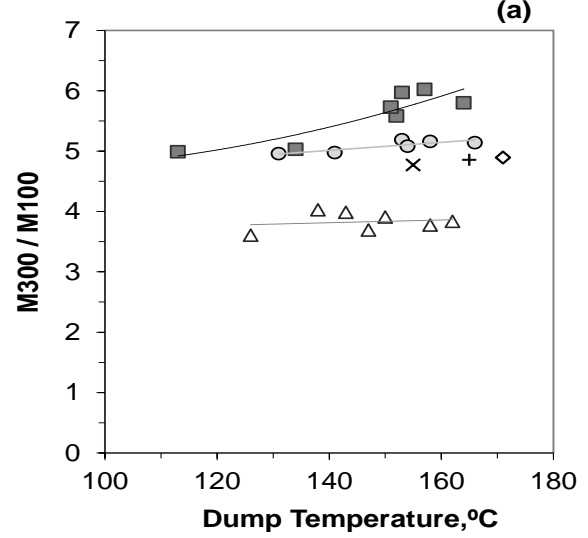

(b)

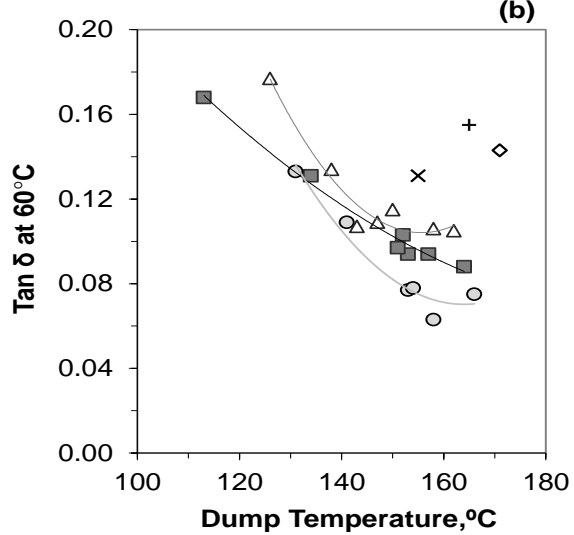

Figure 4.12 Comparison of (a) reinforcement index $M 300 / M 100$ and (b) $\tan \delta$ at $60^{\circ} \mathrm{C}$ for natural rubber vulcanizates with varying protein content; symbols as in Figure 4.10.

\subsection{CONCLUSIONS}

Coupling agent and proteins show a complicated antagonistic effect in silica reinforcement of natural rubber. The effect of proteins is most pronounced when no silane is used in NRsilica compounds. When high amounts of proteins are present in NR, the interactions between proteins and silica are able to disrupt the silica-silica networking. The temperature development is an important parameter in mixing NR-silica with the aid of TESPT as coupling agent, as silica-silica interaction is reduced through silanization at sufficiently high mixing temperatures. This is clearly the case for NR and low protein content rubber DPNR. However, mixing temperature has little influence on the properties of a high protein-content skim rubber compound. Consequently, the hydrophobization of the silica surface by silane may be partially hindered due to silica-protein interactions.

\subsection{REFERENCES}

1. W. Meon, A. Blume, H-D. Luginsland, and S. Uhrlandt, "Silica and Silane", in Rubber Compounding: Chemistry and Applications, B. Rodgers, Ed., Marcel Dekker, New York (2004).

2. J.W. Noordermeer and W.K. Dierkes, "Silica-Filled Rubber Compounds", in Rubber Technologist's Handbook Vol.2, J. White, S.K. De and K. Naskar, Eds., Smithers Rapra Technology, Shawbury, Shrewsbury, Shropshire, UK, (2008).

3. S.S. Sarkawi, W.K. Dierkes and J.W.M. Noordermeer, presented at EU-PEARLS 2010 Meeting: The Future of Natural Rubber, Montpellier, France (2010).

4. A.B. Othman and C. Hepburn, Plast. Rub. Compos. Pro., 19, 185 (1993). 
5. Y. Tanaka and L. Tarachiwin, Rubber Chem. Technol., 82, 283 (2009).

6. L. Tarachiwin, J. Sakdapipanich, K. Ute, T. Kitayama, T. Bamba, E. Fukusaki, A. Kobayashi and Y. Tanaka, Biomacromolecules.,6, 1851 (2005).

7. J. Sakdapipanich, J. Biosci. Bioeng., 103, 287 (2007).

8. A.H. Eng, S. Kawahara and Y. Tanaka, Rubber Chem. Technol., 67, 159 (1993).

9. K. Nawamawat, J. Sakdapipanich, D. Mekkriengkrai and Y. Tanaka, Kautsch. Gummi Kunstst., 61, 518 (2008).

10. D. Mekkriengkrai, J. Sakdapipanich and Y. Tanaka, Rubber Chem Technol., 79, 366 (2006).

11. L. Tarachiwin, J. Sakdapipanich and Y. Tanaka, Kautsch. Gummi Kunstst., 58, 115 (2005).

12. S. Amnuaypornsri, J. Sakdapipanich, S. Toki, B.S. Hsiao, N. Ichikawa and Y. Tanaka, Rubber Chem. Technol., 81, 753 (2008).

13. S. Amnuaypornsri, A. Nimpaiboon, and J. Sakdapipanich, Kautsch. Gummi Kunstst., 62, 88 (2009).

14. M.J.R. Loadman and W.C. Wake, "Analysis of Rubber and Rubber-like Polymers", Kluwer Academic Publishers, the Netherlands, 96 (1988).

15. F.W. Barlow, in: Rubber Compounding: Principles, Materials and Techniques, Chapter 2: Elastomers: Natural Rubber, M. Dekker, New York, (1988).

16. S. Kawahara, W. Klinkai, H. Kuroda and Y. Isono, Polym. Adv. Technol., 15, 181 (2004).

17. W. Klinklai, T. Saito, S. Kawahara, K. Tashiro, Y. Suzuki, J.T. Sakdapipanich and Y. Isono, J. Appl. Polym. Sci., 93, 555 (2004).

18. D.J.C. Salamone, Polymeric Materials Encyclopedia, Vol. 6, CRC Press Inc., 4556 (1996).

19. E.C. Gregg and J.H. Macey, Rubber Chem. Technol., 46, 47 (1973).

20. A. B. Othman and H. Hasma, Proceedings International Rubber Technology Conference 1988, 166 (1988).

21. C.J. Lin, W.L. Hergenrother, E. Alexanian and G.G.A. Böhm, Rubber Chem. Technol., 75, 865 (2002).

22. S. Mihara, R.N. Datta and J.W.M. Noordermeer, Rubber Chem. Technol., 82, 524 (2009).

23. L.A.E.M. Reuvekamp, J.W. ten Brinke, P.J. van Swaaij and J.W.M. Noordermeer, Rubber Chem. Technol., 75, 187 (2002).

24. W.K. Kaewsakul, K. Sahakaro, W.K. Dierkes and J.W.M. Noordermeer, Rubber Chem. Technol., 85, 277 (2012).

25. S. Wolff, Kautsch. Gummi Kunstst., 34, 280 (1981).

26. D.S. Villars, J. Polym. Sci., 21, 257(1956).

27. G. Kraus and J.T. Gruver. Rubber Chem. Technol., 41,1256(1968).

28. B. Meissner, Rubber Chem. Technol., 48, 810 (1975).

29. J.W. ten Brinke, P.J. van Swaaij, L.A.E.M. Reuvekamp and J.W.M. Noordermeer, Rubber 92 Chem. Technol., 76, 12 (2003). 
Chapter 5

\section{Elucidation of Filler-to-Filler and Filler-to-Rubber Interactions in Silica Reinforced Natural Rubber by TEM Network Visualization}

Filler-to-rubber interaction is a key parameter in the reinforcement of rubber. This chapter presents an investigation into filler-to-filler and filler-to-rubber interactions in silica-reinforced Natural Rubber (NR) in the presence and absence of a silane coupling agent. Using a special network visualization technique based on Transmission Electron Microscopy (TEM), insight into the silica and rubber interaction in NR is gained. In absence of silane, vacuoles around the silica particles are formed as a result of a weak filler-to-rubber interaction, while the presence of silane leads to strong filler-to-rubber bonding, which prevents formation of vacuoles. Further, the relationship between the filler-to-rubber interaction as seen from TEM images and bound rubber and Payne effect is discussed. 


\subsection{INTRODUCTION}

Rubber polymers, with Natural Rubber (NR) as the largest representative, need to be reinforced with fillers to provide necessary strength for most applications. In recent years, high-dispersion silica has become the preferred alternative to conventional carbon-black fillers in tire tread compounds. Its use provides considerable improvement in rollingresistance of passenger car tires. However, since silica is highly polar and hydrophilic, it is incompatible with apolar rubbers such as NR. Therefore, a bi-functional silane coupling agent, such as bis(triethoxysilylpropyl) tetrasulfide (TESPT) is needed to improve the fillerto-rubber interaction on the nano-scale by creating chemical links between the silica surface and the rubber chains ${ }^{1,2}$.

The dynamic modulus of rubber increases with addition of reinforcing filler. The contributions of the fillers to the modulus of rubber can be divided into two: strain-dependent and strain-independent parts. Filler-filler interaction contributes to the strain-dependence of the modulus. The modulus decreases with increasing strain: the so-called 'Payne effect', which is due to a partially reversible breakdown of the filler network ${ }^{3}$. The strain-independent part of the modulus is a combination of filler-rubber interactions, the crosslinked rubber network contribution and a hydrodynamic effect of the filler as illustrated in Figure 5.1 $1^{4-6}$. Filler-filler and filler-rubber interactions are two partially competitive processes. A decrease in filler networking would lead to better filler-rubber interaction. Filler-filler interaction is commonly characterized by the Payne effect: the drop in storage modulus in a dynamic mechanical test when the strain (deformation) is increased from low $(<1 \%)$ to a high value $(100 \%)$ at constant frequency and temperature. The use of TESPT significantly reduces the Payne effect in silica-filled compounds as a result of hydrophobation of the silica surface and therefore increased filler-rubber bonds. The phenomenon of bound rubber is another evidence for filler-rubber bonds, which has been studied by many scholars ${ }^{7-11}$. This rubberto-filler interaction is reflected macroscopically in the formation of bound rubber, which involves physical adsorption, chemisorption and mechanical interaction ${ }^{10,12}$.

Transmission Electron Microscopy (TEM) was used by Julve et al. to evaluate the microdispersion of silica in a silica tire tread compound with a high filler content ${ }^{13}$. Reuvekamp found that a better silica microdispersion is obtained at high discharge temperature after mixing, as characterized by an increase in the amount of segregated silica particles $^{14}$. A technique called Network Visualization using TEM is a beneficial tool to study the rubber to filler interaction in rubber compounds. This technique was first developed at the Tun Abdul Razak Research Centre (TARRC) to study rubber networks and was later used to investigate rubber-to-filler interactions ${ }^{15}$. The TEM Network Visualization process 94 
involves swelling of a crosslinked vulcanizate in styrene, polymerization of the styrene, staining the rubber network and visualization using $\mathrm{TEM}^{16}$. Using this method, the topology of a rubber network can be identified from the stained elastomer moieties and the unstained polystyrene matrix.

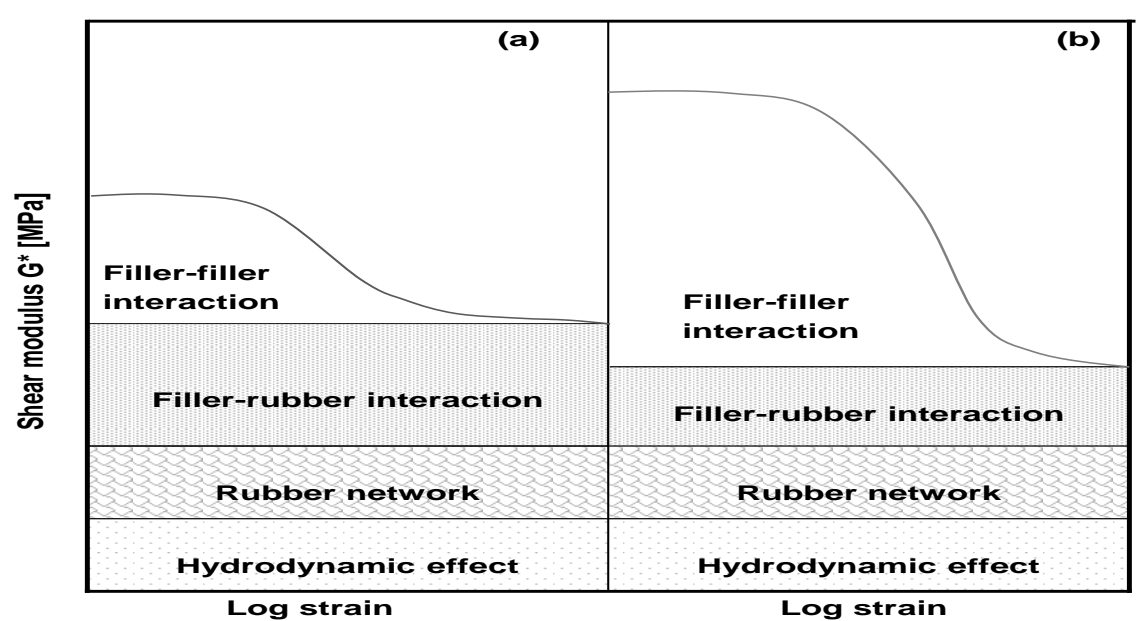

Figure 5.1 Contributions to the shear modulus of filled rubber (a) carbon black filled rubber (b)silica filled rubber.

Chapman and coworkers used the TEM Network Visualization technique to investigate the rubber-to-filler interaction between NR and 25 mole\% Epoxidized NR (ENR25) vulcanizates filled with 20 phr silica ${ }^{16,17}$. Polystyrene void regions between the silica and rubber are clearly visible for the NR-silica vulcanizate. In contrast, the ENR-25 network is fully attached to the silica particles and no voids are seen, indicating a higher rubber-tofiller affinity for the ENR-silica vulcanizate. However, for NR there are a small number of stained network strands connecting the silica particles and the NR network which indicates that there still is a certain interaction between NR and silica.

The present chapter presents an investigation into filler-to-rubber interaction in silica reinforced NR in the presence and absence of a silane coupling agent. The filler-filler interactions are monitored by measuring the Payne effect. The TEM Network Visualization technique is used to look at the filler/rubber morphology and to obtain further insight into the reinforcing mechanism of silica in NR. In addition, the influence of proteins contained in the rubber on the silica-NR interaction is studied by using NR with different protein contents. The relationship of the filler-to-rubber interactions as seen from the TEM images with bound rubber and Payne effect measurements is further detailed. 


\subsection{EXPERIMENTAL}

\subsubsection{Materials and compounding}

In the present experiments, the ingredients and compound formulation were the same as what has been described in Chapter 4. The compounds were mixed and vulcanized according to the experimental section of Chapter 3 .

\subsubsection{Sample preparation for TEM Network Visualization}

Samples were taken from the vulcanizates and extracted overnight using acetone to remove remaining curing additives. A strip of approx. $10 \mathrm{~mm} \times 5 \mathrm{~mm}$ from the extracted vulcanizate sample was then swollen in a styrene solution ${ }^{16,17}$ containing a radical initiator (1 wt. \% Benzoyl Peroxide, 2 wt. \% Dibutyl Phthalate plasticizer) for 2 days. A 10mm x 2mm strip was then cut from the swollen sample and transferred into a polymer capsule. The capsule was filled up with styrene solution and fitted with a cap. The capsule was heated overnight at $68^{\circ} \mathrm{C}$ for the styrene to be polymerized. Only when the capsules had hardened fully, could they be subjected to microscopy. Depending on the sample, it was sometimes necessary to 'post cure' the capsules in an oven at $90^{\circ}$ for several hours to completely harden the styrene.

\subsubsection{Characterization methods}

Payne effect and bound rubber content (BRC) were measured according to the methods as described in Chapter 3. The amount of BRC (\%) was calculated as:

$$
\text { BRC }(\%)=\left(\frac{w_{\text {dry }}-w_{\text {insolubles }}}{w_{o} \times \frac{100}{w_{\text {total }, p h r}}}\right) \times 100 \%
$$

where $w_{0}$ is the initial weight of the sample, $w_{\text {dry }}$ is the dry weight of the extracted sample, $W_{\text {insolubles }}$ is the weight of insoluble matter (mainly filler) in the samples, and $w_{\text {total, phr }}$ is the total compound weight in phr (parts per hundred rubber). In this study, total BRC refers to $B R C$ if measured in a normal atmosphere, while chemical BRC refers to BRC measured in an ammonia environment ${ }^{10,19}$.

The apparent crosslink density was determined by swelling of a vulcanized sample in toluene according to the method as described in Chapter 4. The apparent crosslink density was calculated as the reciprocal swelling value, $1 / \mathrm{Q}$.

\section{Measurements of glass transition temperature}

The glass transition temperature ( $\mathrm{Tg}$ ) was measured using a Metravib Dynamic Mechanical Analyzer (DMA) viscoanalyzer VA 2000. The samples were cut from the vulcanized sheets 
of $2 \mathrm{~mm}$ thickness. A temperature sweep measurement from -80 to $+80^{\circ} \mathrm{C}$ was performed in tension mode at a frequency of $10 \mathrm{~Hz}$ and dynamic strain of $0.1 \%$.

\section{Transmission Electron Microscopy (TEM)}

TEM analysis of a swollen rubber sample embedded in the polymerized polystyrene matrix was done using a Philips CM12 TEM operating at $80 \mathrm{kV}$ at the Tun Abdul Razak Research Centre (TARRC), Hertford, United Kingdom. An ultra-thin section of the sample was obtained by ultramicrotomy at room temperature using glass knives. The microtome used was PowerTome PC (RMC). The sections were collected in a water-filled trough and relaxed with xylene vapour before collecting on TEM grids. The sections were stained with osmium tetroxide ${ }^{15-17}$ vapor for one hour. Osmium tetroxide reacts with carbon-carbon double bonds and this results in the rubber network appearing darker than the polystyrene. By using this method, the regions of rubber network can be identified from the stained rubber and unstained polystyrene matrix.

\subsection{ResULTS AND Discussion}

\subsubsection{Influence of silane and proteins on filler-filler interactions}

Filler-filler interaction is commonly measured by the Payne effect. A comparison of filler-filler interaction in the NR-silica compounds in both the absence and presence of silane coupling agent TESPT, is shown in Figure 5.2. The Payne effect of silica compounds with TESPT is noticeably lower than for compounds without TESPT, except for skim rubber. This indicates that the hydrophobation of the silica surface by TESPT greatly reduces the filler-filler interaction. The influence of non-rubber constituents in the NR, in particular proteins, on silica-silica interaction can also be seen, where the low protein DPNR compound exhibits a higher Payne effect and the high protein skim rubber compound has the lower Payne effect. The proteins in NR have a similar effect as silane in hydrophobizing the silica surfaces, although not as strong, as shown in Figure 5.3(a). For unvulcanized samples, the Payne effect for compounds without silane decreases with increasing content of proteins in the NR. The role of the proteins is to disrupt the silica-silica network resulting in lower filler-filler interaction. The physical interaction between silica and proteins through hydrogen bonding with functional protein groups is illustrated in Figure 5.4.

This effect is not seen in the compounds with TESPT, as silica-TESPT coupling apparently is much stronger than the silica-protein interaction. Interestingly, with a lot of proteins present in the rubber, the Payne effect for the compound shows no difference whether or not silane is used. 


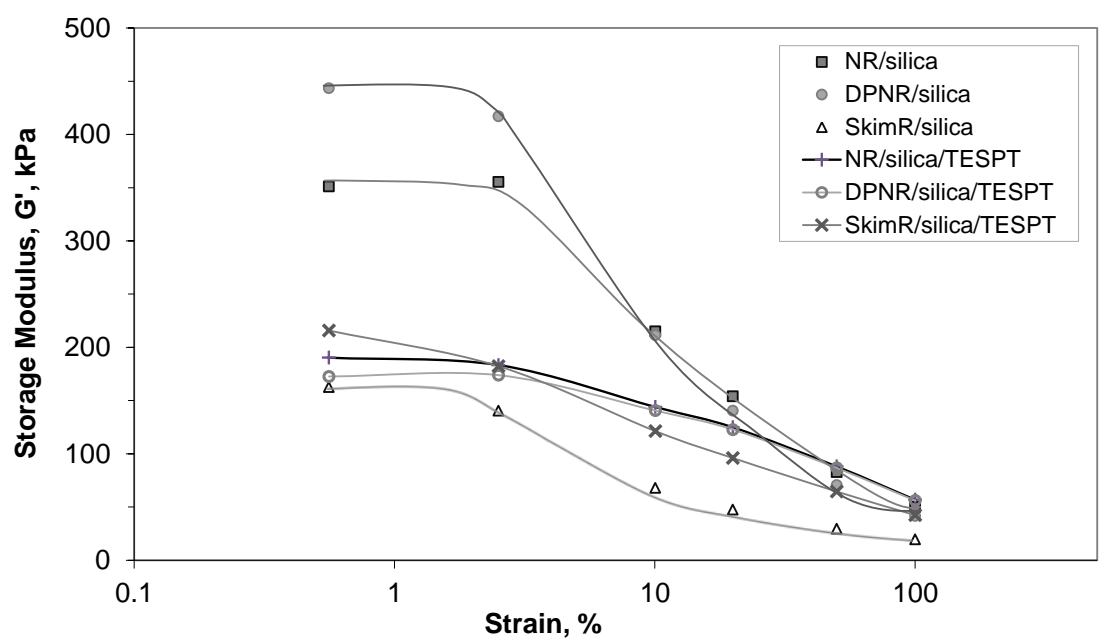

Figure 5.2 Payne effect of silica compounds as influenced by silane and protein contents in NR.

The Payne effect measurements performed on the vulcanized compounds show opposite behavior. In Figure 5.3(b), the Payne effect for the vulcanized compounds with TESPT increases with increasing protein content in NR. The lower filler-filler interaction in the DPNR compound compared to NR indicates that there is more coupling between TESPT and silica with less protein present or purified NR.
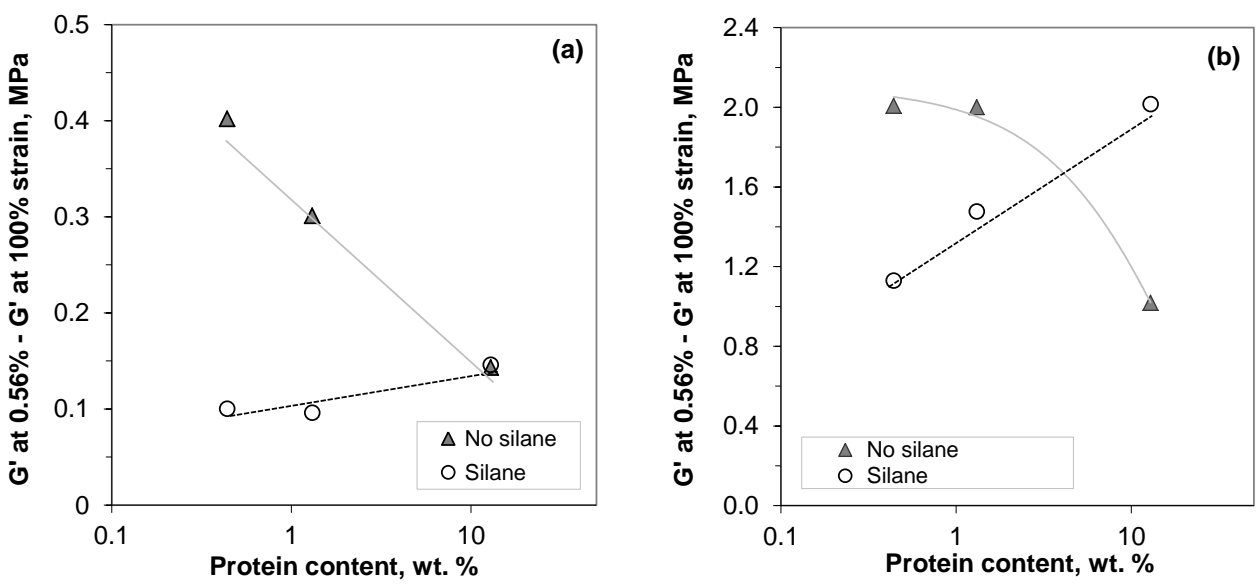

Figure 5.3 Comparison of Payne effect between silica-NR compounds with varying protein contents in the presence and absence of silane; (a): unvulcanized samples; (b): vulcanized samples. 
The difference in the Payne effect between NR and DPNR is also seen in the compound without silane. Nonetheless, the Payne effect of the NR and DPNR compound with silane is still considerably lower than for compounds without silane. As observed with the unvulcanized samples, the protein-silica interactions in the now vulcanized sample of skim rubber without silane also results in lower filler-filler interaction. What remains puzzling is the opposite effect when silane is present in the skim rubber compound. It seems that there is a competitive effect between protein and silane in the hydrophobation of the silica surface. Another possible explanation is the denaturation of protein after vulcanization, which results in the breakage of silica-proteins interaction and consequently gives rise to a silica-silica network to reform.
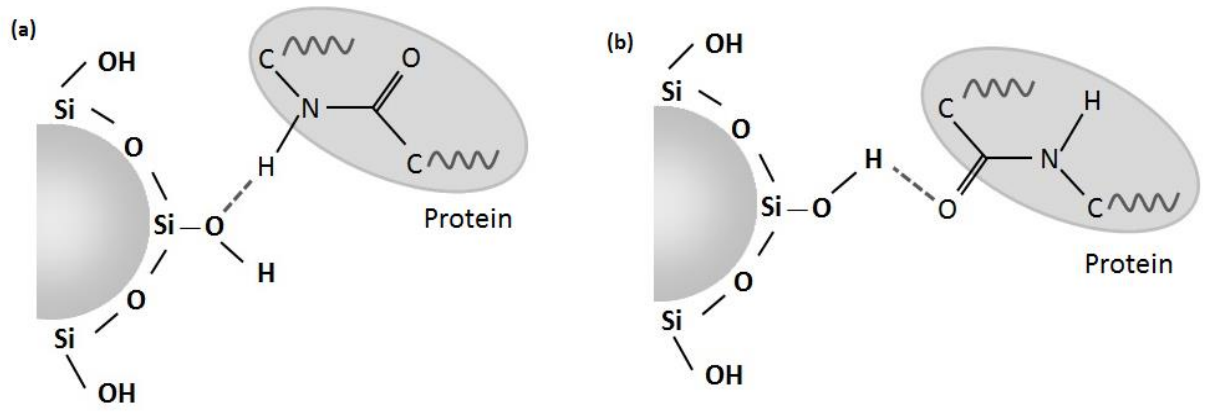

Figure 5.4 Illustration of possible interactions between silanol groups of silica and proteins in natural rubber via hydrogen bonding with functional groups. (a)amino group (b) carboxyl group.

\subsubsection{Bound rubber and filler-to-rubber interactions in silica compounds}

Bound rubber is the polymer portion that remains bound to the filler when an unvulcanized compound is extracted with a good solvent such as toluene. For ease of description, the bound rubber can be described according to its layer on the filler particle or aggregate, resulting in a tightly bound rubber skin and a loosely bound rubber shell. In the present study, the total BRC as measured in normal atmosphere is a combination of the tightly and the loosely bound rubber. The chemically BRC as obtained from extraction in an ammonia atmosphere is only tightly chemically bound rubber left, as the loosely physically bound rubber is also extracted. As proposed by Wolff ${ }^{10}$, there are three configurations of rubber chain attachments to a filler particle as depicted in Figure 5.5, which can be described as follows: 
(a) single attachment, where only one chain segment is immobilized on a filler particle;

(b) multiple attachment of several chain segments from one rubber chain;

(c) inter-particle attachment where one rubber chain connects to two or more filler particles. Bound rubber can only be formed if the inter-particle attachment or configuration (c) exists in the system as configurations (a) and (b) will lead to disperse gel, and determination of BRC would be impossible with the common bound rubber procedure. The inter-particle attachment in bound rubber is also in agreement with the morphology described by Leblanc $^{11}$ and Papon et al. ${ }^{20}$.

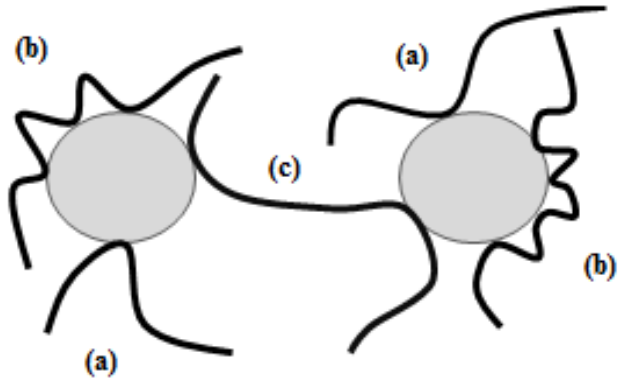

Figure 5.5 Illustration of adsorption of rubber chains on filler particles leading to rubber-tofiller interaction: (a) single attachment, (b) multiple attachment; and (c) inter-particle attachment.

Table 5.1 shows a comparison of BRC between silica compounds both with and without silane. Most of the BRC formed in a NR-silica-TESPT compound is chemically bound. This is obviously due to the hydrophobation of the silica surface as a result of silanization with TESPT. The increase in silica-TESPT coupling reduces the specific component of surface energy, $\gamma_{s}^{\text {sp }}$ of silica and consequently results in more filler-to-rubber interaction. This corresponds well with the lower Payne effect of the silica compounds with TESPT. Without silane, the silica compounds still form bound rubber, as indicated by the total BRC. However, no chemically BRC was obtained for the silica compounds without silane after ammonia treatment. This indicates that silica compounds without silane have weak interaction with rubber due to the high $\gamma_{s}^{\text {sp }}$ of silica which leads to a stronger filler-filler network, as reflected in the high Payne effect as shown in Figure 5.2. 
Table 5.1 Comparison of bound rubber content (BRC) of silica compound with silane and without silane

\begin{tabular}{lcccc}
\hline Rubber & \multicolumn{2}{c}{ Without silane } & \multicolumn{2}{c}{ With silane } \\
\cline { 2 - 5 } types & $\begin{array}{c}\text { Physically } \\
\text { BRC [\%] }\end{array}$ & $\begin{array}{c}\text { Chemically } \\
\text { BRC [\%] }\end{array}$ & $\begin{array}{c}\text { Physically } \\
\text { BRC [\%] }\end{array}$ & $\begin{array}{c}\text { Chemically } \\
\text { BRC [\%] }\end{array}$ \\
\hline NR & 57 & 0 & 11 & 68 \\
DPNR & 45 & 0 & 11 & 76 \\
Skim Rubber & 51 & 0 & 13 & 80 \\
\hline
\end{tabular}

Ammonia treatment of bound rubber was first described by Polmanteer and Lentz ${ }^{19}$ to chemically break polymer-filler bonds in silica-filled silicone rubber. In their work, the silicone rubber fraction attached to the silica surface by hydrogen bonds between free silanol groups on the filler structure and siloxane bonds of the elastomer were dissolved by toluene in an ammonia atmosphere. In the case of the NR and silica system without silane, filler-to-rubber interaction is not due to hydrogen bonds but more based on physical adsorption and mechanical interaction. In comparison, a carbon black compound shows very little difference in bound rubber with or without ammonia treatment: Table 5.2. This phenomenon was also reported by Wolff et al. ${ }^{10}$ and Choi and $\mathrm{Kim}^{21}$. It is well accepted that the bound rubber in carbon black-filled rubber is mainly caused by adsorption processes and at the most only $23 \%$ of the bound rubber is attributed to covalent bonds between rubber and carbon black.

Table 5.2 Comparison of BRC of a carbon black compound ${ }^{\mathrm{a}}$ in normal and ammonia atmosphere

\begin{tabular}{|c|c|}
\hline & $\operatorname{ber}(\%)$ \\
\hline Normal & 46 \\
\hline Ammonia atmosphere & 44 \\
\hline
\end{tabular}

The present results point to the interesting fact that the ammonia treatment of bound rubber only works for silica compounds. Hydrogen bonds in silica-filled NR without silane come mostly from inter-particle silica-silica interactions. With ammonia treatment, the hydrogen bonds in the silica-silica interactions are destroyed as schematically shown in Figure 5.6. This results in the disperse gel and consequently no chemically BRC is obtained. 
Furthermore, it explains that in the silica-filled skim-rubber compound there is no chemically $B R C$, even though in this case the silica-silica network is disrupted by proteins, and the interaction between silica and proteins is mainly via hydrogen bonding again: Figure 5.4. This finding demonstrates that the filler-filler interaction or filler morphology is one of the major factors contributing to the bound rubber formation for untreated silica filler. In addition, a lower Payne effect or less filler-filler interaction does not necessarily lead to an increase in rubber-to-filler interaction as demonstrated in the skim rubber-silica compound without silane.
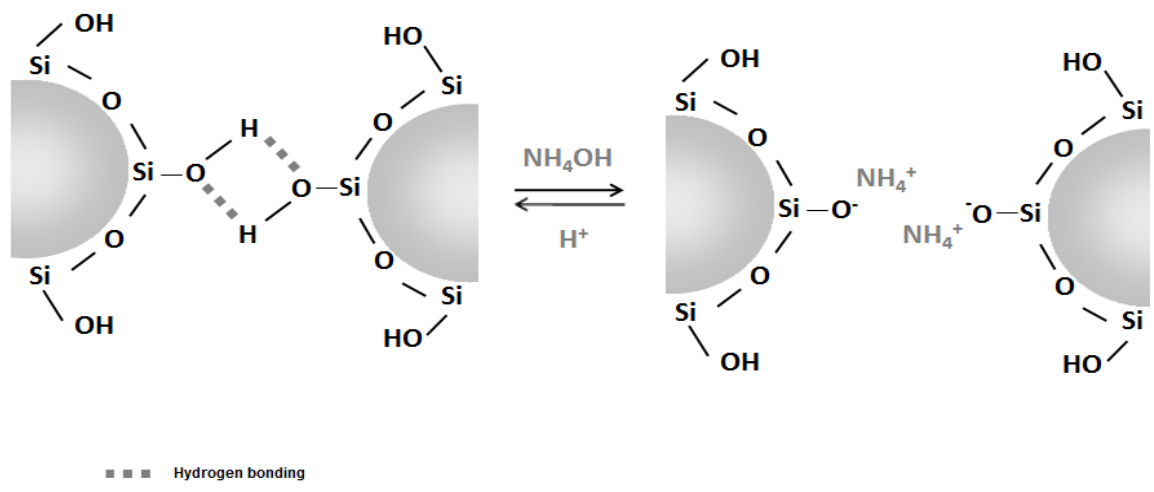

Figure 5.6 Proposed mechanism for the destruction of hydrogen bonding during bound rubber content measurement with ammonia treatment.

\subsubsection{TEM Network Visualization of silica-NR compounds}

Attempting to analyze the morphology of filler-to-rubber interaction in silica compounds at high loading, which in this study is 55 phr of silica, is difficult as the silica aggregates are very close together as depicted in the TEM image in Figure 5.7(a). In order to gain insight into the filler-to-rubber interaction, TEM network visualization was carried out where the vulcanizate was swollen in styrene. The comparison of silica morphology after swelling in styrene is shown in Figure 5.7(b) where the distances between the silica aggregates are enlarged. According to Ladouce-Stelandre et al. $^{15}$ the swelling ratio of the vulcanizate swollen in styrene monomer is close to three, and the force is equally distributed in the three dimensions of the sample. Hence, it should be noted that the images of the styrene swollen compounds are not representative of the dispersion of silica in the vulcanizates, but are rather meant to look more closely into the interaction between filler and rubber. 

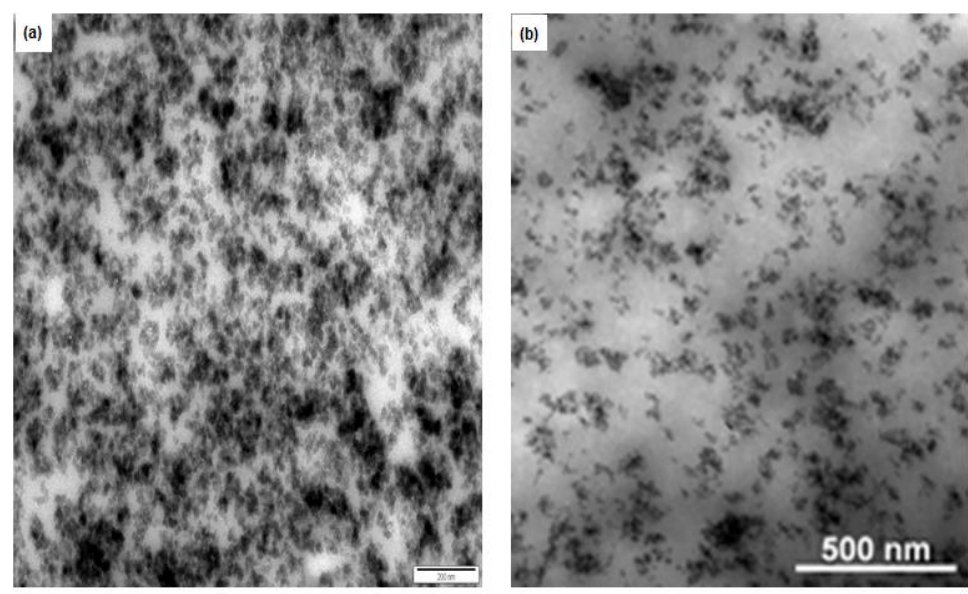

Figure 5.7 Comparison of TEM image for NR-silica-TESPT vulcanizate (a) not swollen with styrene and (b) swollen with styrene

TEM network visualizations of silica-filled NR and silica-filled DPNR vulcanizates without silane coupling agent are depicted in Figures 5.8 and 5.9, respectively. In both micrographs, silica aggregates of around $50-100 \mathrm{~nm}$ size can be seen as dark particles throughout the sample. The vulcanized rubber network appears as a stained mesh structure, while polystyrene appears as unstained regions. Some silica aggregates in the NR vulcanizate are well-bonded to the NR network as seen from the network strands connecting silica particles to the NR network. In addition, there are vacuoles or voids surrounding the silica aggregates. The network visualization of the DPNR vulcanizate is markedly different from the NR vulcanizate. There are clearly vacuoles surrounding the silica aggregates in the DPNR vulcanizate.

The styrene polymerizes between the rubber network and the silica particles, and this results in the polystyrene vacuoles surrounding the silica particles or aggregates. Ladouce-Stelandre et al. have suggested that the formation of such vacuoles is due to a weak interface between silica particles and rubber chains ${ }^{15}$. Since silica exhibits a low dispersive component of surface energy, $\gamma_{s}^{d}$, the filler-to-rubber interaction is weak and not many rubber chains adsorb on its surface. Once a segment of a rubber chain is attached to the silica surface, it is possible that multiple attachment can occur due to segmental reptation of the rubber chain. Both single and multiple attachments or configurations (a) and (b) in Figure 5.5 will lead to formation of vacuoles when the vulcanizate is swollen in styrene. There are less vacuoles present in the NR vulcanizate as compared to the DPNR vulcanizate without silane, which suggests higher filler-to-rubber interactions in the former. 


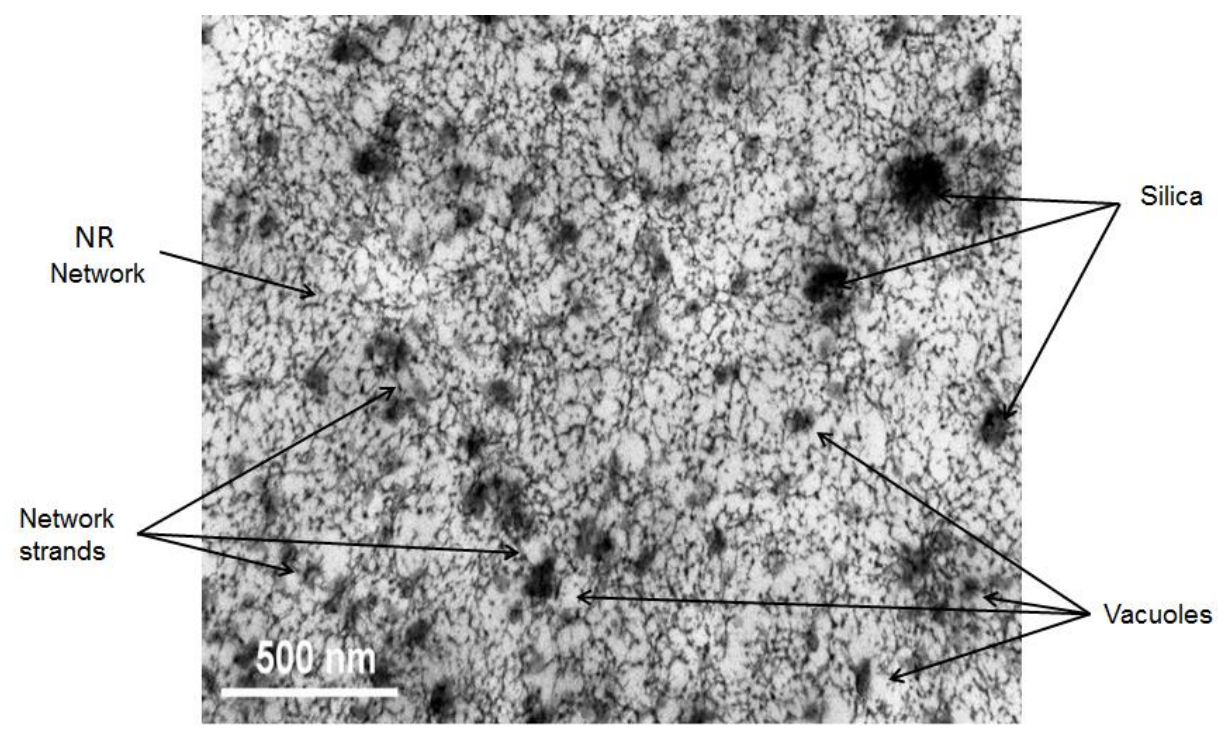

Figure 5.8 TEM Network Visualization micrograph of silica-filled NR vulcanizate without coupling agent

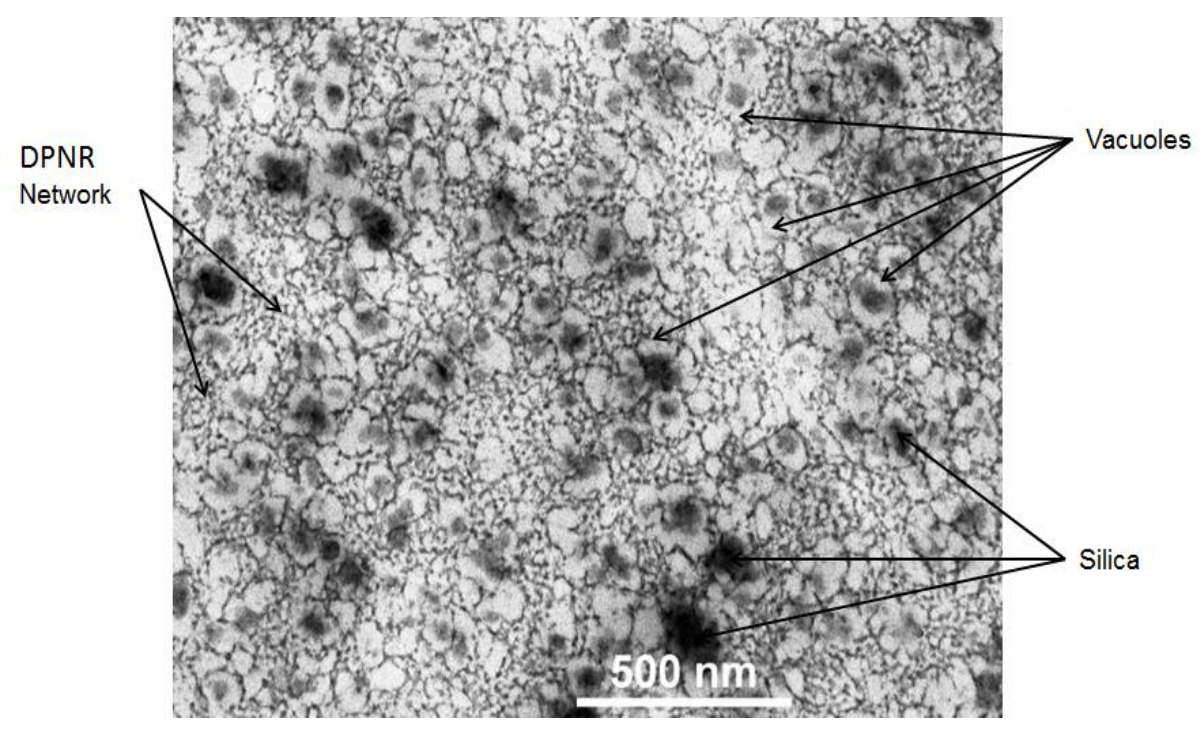

Figure 5.9 TEM Network Visualization micrograph of silica-filled DPNR vulcanizate without coupling agent 


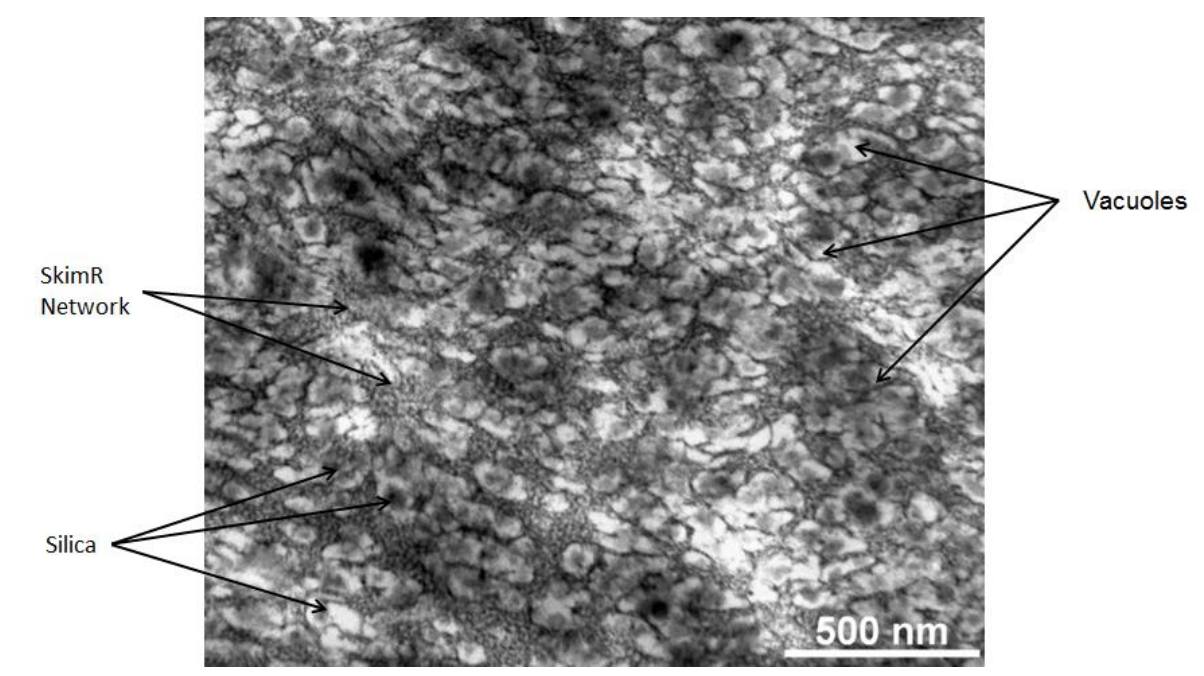

Figure 5.10 TEM Network Visualization micrograph of silica-filled skim rubber vulcanizate without coupling agent

Figure 5.10 shows the TEM network visualization of the skim rubber-silica vulcanizate without coupling agent. The size of the silica aggregates in the skim rubber matrix is smaller as compared to those in the NR and DPNR vulcanizates without silane. This is in agreement with the earlier results of the Payne effect which demonstrated that silica-silica interactions were disrupted by the high amount of protein present in the skim rubber. However, vacuoles surrounding the silica particles and aggregates are clearly observed in the skim-rubber-silica vulcanizate. The existence of such vacuoles in the vulcanizate indicates again weak interaction between the silica and the skim rubber. This is confirmed by no chemically BRC as obtained for skim rubber without silane. As discussed earlier, the lower Payne effect in skim rubber is not resulting in higher filler-to-rubber interaction. The skim rubber network also appears to be different from the NR and DPNR networks, where the mesh is smaller. It may be due to the lower molecular weight of skim rubber compared to NR and also to a higher crosslink density of skim rubber than for NR and DPNR without silane (Figure 5.11).

A comparison of the TEM network visualization between NR, DPNR and skim rubber vulcanizates with TESPT coupling agent included is shown in Figures 5.12, 5.13 and 5.14 , respectively. Even though the micrographs are relatively less clear, it can be seen that there is strong attachment of the rubber network to the silica aggregates in all three rubbers with TESPT present. Voids are scarcely visible in the TEM images. The aggregates of silica 
are also smaller as compared to those in vulcanizates without silane. This agrees well with the earlier data that all silica compounds with TESPT exhibited a lower Payne effect and very high chemically $B R C$. This also shows that these compounds have a high silanization efficiency as a result of good mixing and reaction. In addition, the rubber networks in the vulcanizates with silane appear to be more dense as compared to those without silane. This correlates with the higher apparent crosslink density of the vulcanizates with silane: Figure 5.11 .

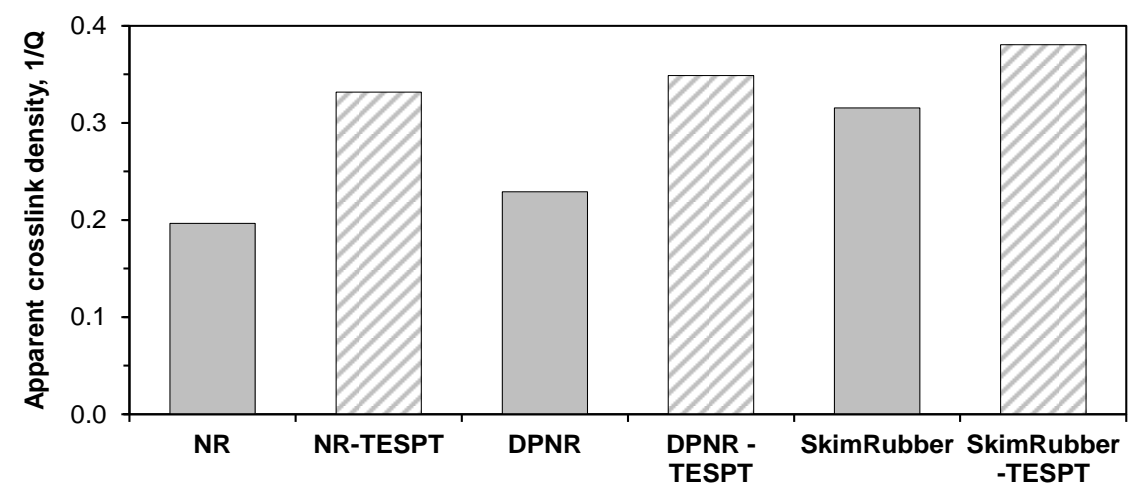

Figure 5.11 Apparent crosslink density of silica vulcanizates without silane and with TESPT silane coupling agent.

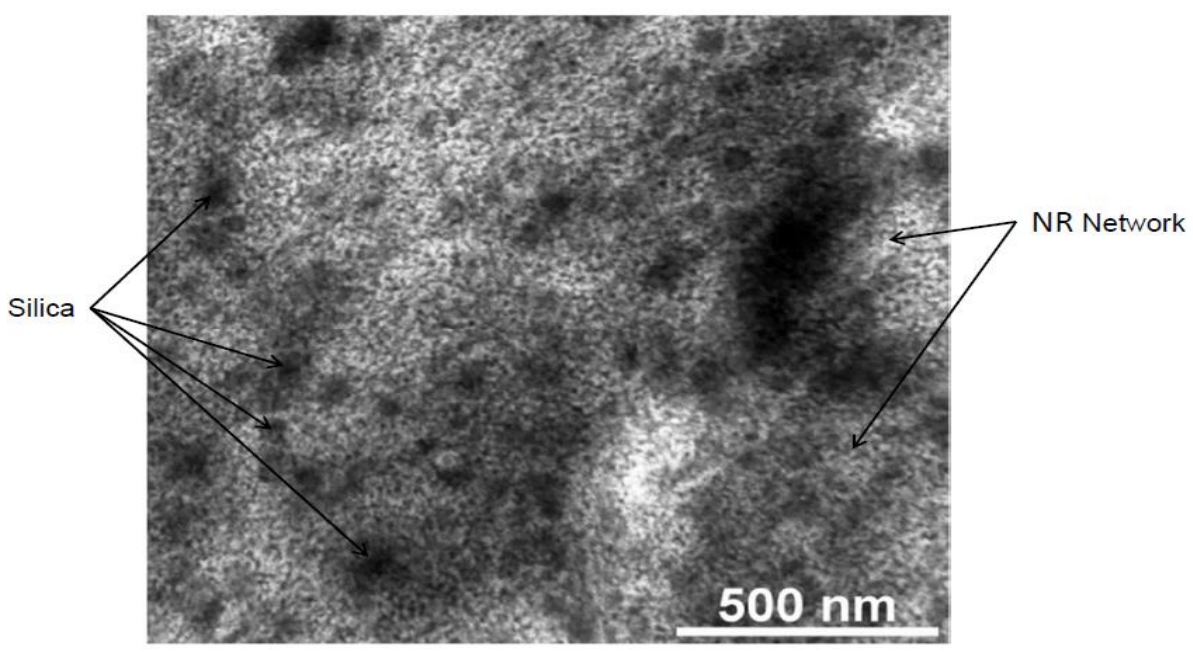

Figure 5.12 TEM Network Visualization micrograph of silica-filled NR vulcanizate with TESPT silane coupling agent. 


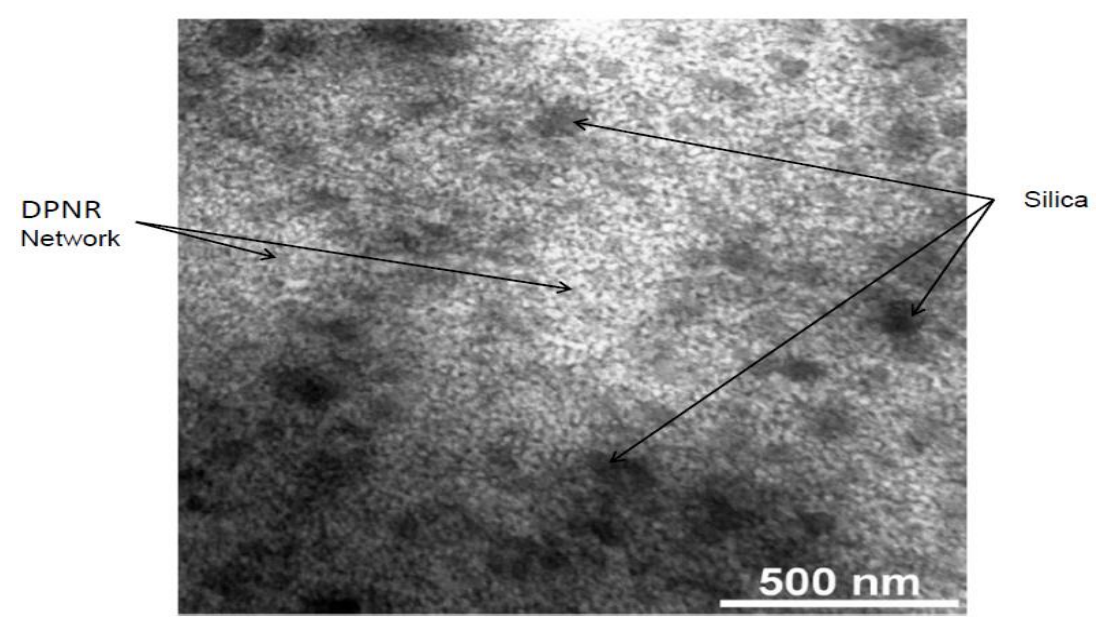

Figure 5.13 TEM Network Visualization micrograph of silica-filled DPNR vulcanizate with TESPT silane coupling agent.

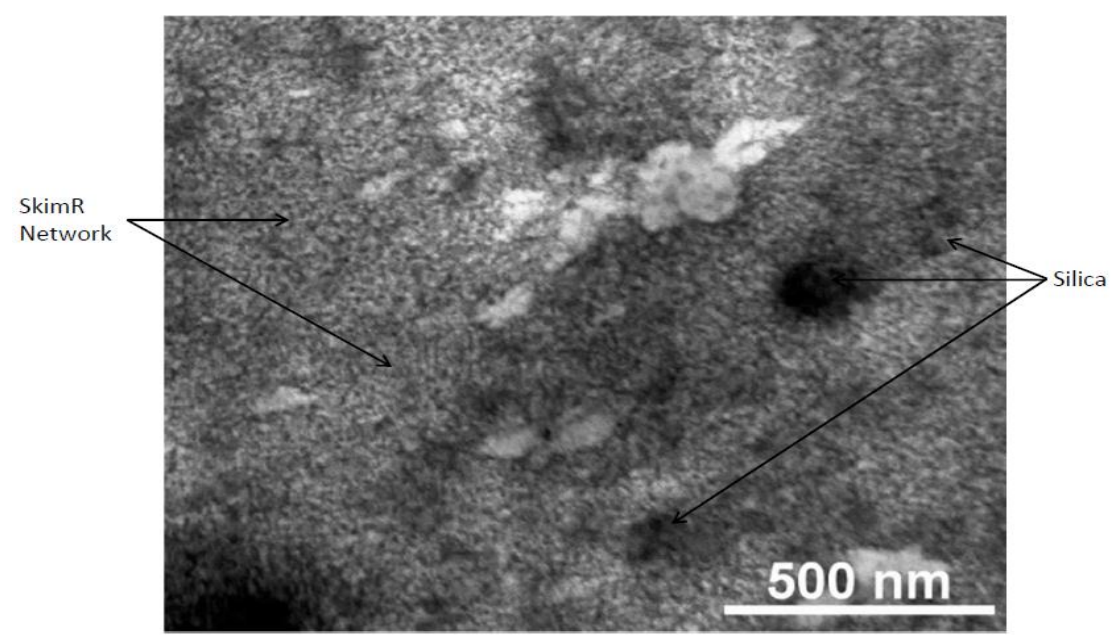

Figure 5.14 TEM Network Visualization micrograph of silica-filled skim rubber vulcanizate with TESPT silane coupling agent.

That the silica-TESPT-rubber bonds created during vulcanization improve the rubber-to-filler interaction, is further supported by a shift in the glass transition temperature ( $\mathrm{Tg}$ ) of vulcanizates with the use of silane TESPT. In Figure 5.15, the $\mathrm{Tg}$ of the vulcanizates, as measured with DMA at $10 \mathrm{~Hz}$, increases from $-49.5^{\circ} \mathrm{C}$ to $-46.5^{\circ} \mathrm{C}$ when TESPT is used, indicating a restriction of the local mobility of rubber chains due to silica-TESPT-rubber 
coupling. In addition, the apparent crosslinking density in vulcanizates with TESPT is higher as indicated in Figure 5.11, due to free sulfur released from TESPT, which also contributes to the increase in $\mathrm{Tg}$. The $\mathrm{Tg}$ is not affected by the non-rubber constituents in the rubber. This is in good agreement with the earlier observation that the interaction of protein and silica is weaker than the silica-TESPT coupling.

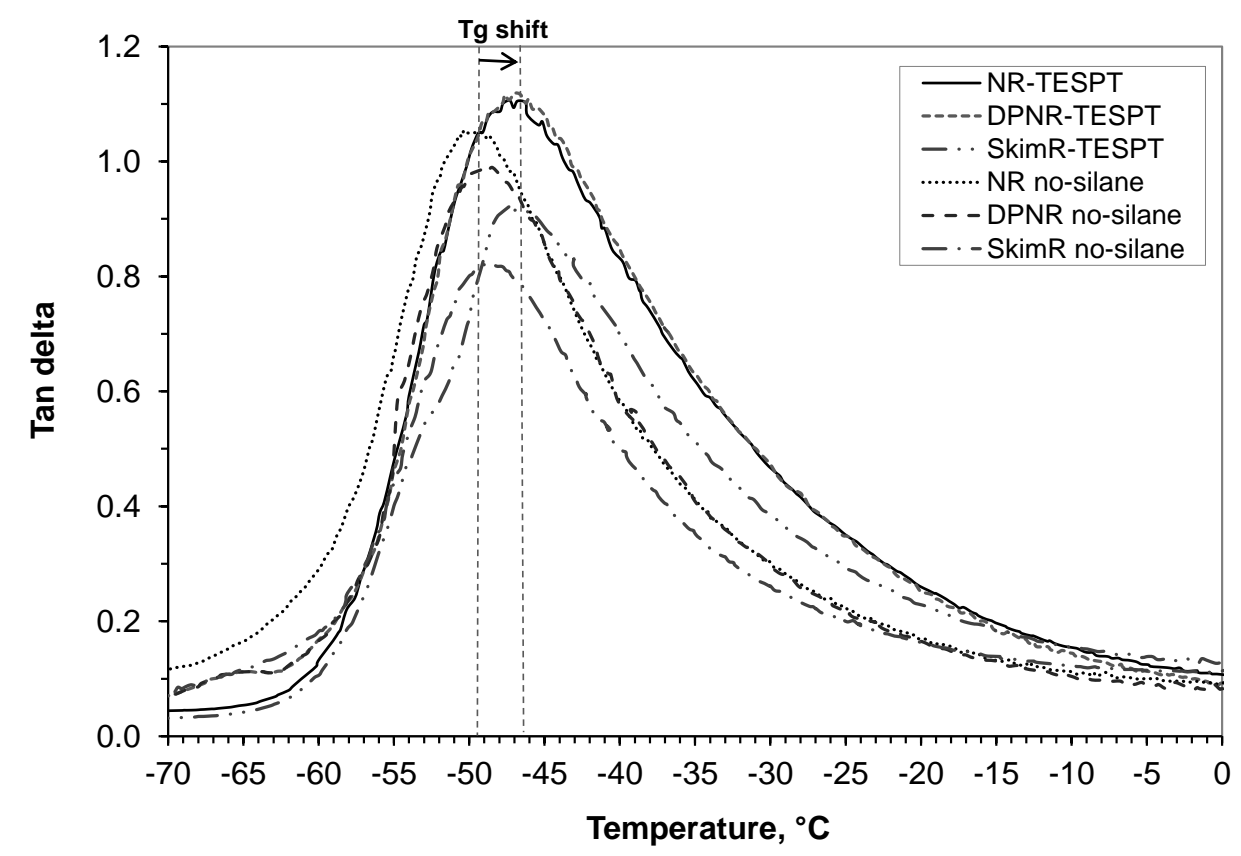

Figure 5.15 The effect of silane TESPT on the glass transition temperature, Tg of silica-

filled vulcanizates

\subsection{CONCLUSIONS}

The rubber-to-filler interaction in silica vulcanizates in the absence of silane is a physical phenomenon. The TEM Network Visualization of vulcanizates without silane reveals vacuoles around the silica particles and aggregates, which indicates weak filler-to-rubber interactions. This is further supported by only physically bound rubber in the compounds without silane. In comparison, the vacuoles are more clearly observed in DPNR and skim rubber-vulcanizates without silane than in the NR-vulcanizate. The rubber-to-filler interaction of silica-filled natural rubber vulcanizates is greatly improved with the use of silane TESPT as coupling agent. The TEM Network Visualization of the silica-vulcanizates with TESPT shows no formation of vacuoles, which demonstrates strong attachment of the rubber 
networks to silica aggregates. This is the result of a chemical reaction between silica and TESPT, as also demonstrated by a high chemically bound rubber content and a low Payne effect.

For NR and DPNR vulcanizates without silane, strong filler-filler interactions as observed from the higher Payne effect contribute to their physically bound rubber. In contrast, skim rubber vulcanizates exhibit lower Payne effects due to disruption of the silicasilica network by proteins. Even though proteins are capable to hydrophobize the silica surface and reduce the filler-filler interaction, they do not contribute to strong rubber-to-filler interactions, as the silica-protein interaction only leads to formation of physically bound rubber.

The present findings from the TEM Network Visualization and shift in the glass transition temperature confirm a strong rubber-to-filler interaction in the silica compounds with silane, and a minimal influence of protein in the presence of silane.

\subsection{REFERENCES}

1. W. Meon, A. Blume, H-D. Luginsland and S. Uhrlandt, "Silica and Silane", in Rubber Compounding: Chemistry and Applications, (Ed. B. Rodgers), Marcel Dekker Inc., New York (2004).

2. J. W. M. Noordermeer and W. K. Dierkes, "Silica-Filled Rubber Compounds", in Rubber Technologist's Handbook, Vol.2, (Eds.: J. White, S. K. De and K. Naskar), Smithers Rapra Technology, Shawbury, Shrewsbury, Shropshire, UK (2008).

3. A. R. Payne and R. E. Whittaker, Rubber Chem. Technol., 44, 440 (1971).

4. J-B. Donnet, Rubber Chem. Technol., 71, 323 (1998).

5. S. Wolff, and M.-J. Wang, Rubber Chem. Technol., 65, 329 (1992).

6. H-D. Luginsland, J. Fröhlich and A. Wehmeier, Rubber Chem. Technol., 75, 563 (2002).

7. C. M. Blow, Polymer, 14, 309 (1973).

8. B. Meissner, Rubber Chem.Technol., 48, 810 (1975).

9. S. Wolff, Rubber Chem. Technol., 69, 325 (1996).

10. S. Wolff, M.-J. Wang and E-H. Tan, Rubber Chem. Technol., 66, 163 (1993).

11. J. L. Leblanc, J. Appl. Polym. Sci., 66, 1541 (2000).

12. J. L. Leblanc, Prog. Polym. Sci., 27, 627 (2002).

13. D. Julve, M. Menendez, J. Perez and J. Ramos, Rubber Chem. Technol., 69, 74 (1996).

14. L. A. E. M Reuvekamp, PhD Thesis, University of Twente, Enschede, the Netherlands, (2003).

15. L. Ladouce-Stelandre, Y. Bomal, L. Flandin and D. Labarre, Rubber Chem.Technol., 76, 145 (2003). 
16. A. V. Chapman, presented at 24th International H.F. Mark-Symposium, 'Advances in the Field of Elastomers \& Thermoplastic Elastomers', Vienna (2007).

17. S. Cook, A. V. Chapman, A. J. Tinker and L. Oleksik, presented at Fall 172th ACS Rubber Division Meeting, Cleveland, OH (2007).

18. M. J. R. Loadman and W.C. Wake, Analysis of Rubber and Rubber-like Polymers, Kluwer Academic Publishers, the Netherlands (1988).

19. K. E. Polmanteer and C. W. Lentz, Rubber Chem. Technol., 48, 795 (1975).

20. A. Papon, H. Montes, F. Lequeux, J. Oberdisse, K. Saalwächter and L. Guy, Soft Matter, 8, 4090 (2002).

21. S. -S. Choi, and I. -S. Kim, Eur. Pol. J., 38, 1265 (2002). 


\section{Chapter 6}

\section{A Morphological Study of Macro- and Micro-Dispersion of Silica in Natural Rubber Vulcanizates}

A good dispersion of silica in a rubber vulcanizate is important as it influences the final properties. Macro- and micro-dispersion morphologies of silica in Natural Rubber (NR), Deproteinized Natural Rubber (DPNR) and skim rubber vulcanizates are investigated by combining several different morphological techniques. Reflective light microscopy and Scanning Electron Microscopy (SEM) are used to analyze the macro-dispersion of silica. The micro-dispersion of silica is further evaluated using Atomic Force Microscopy (AFM) and Transmission Electron Microscopy (TEM). The results show that the use of coupling agent TESPT enhances the micro-dispersion of silica, which results in an increase of filler-torubber interactions. In the absence of a silane coupling agent, a similar micro-dispersion is observed for NR and DPNR vulcanizates from AFM phase images. Silica networking is clearly very strong in skim rubber vulcanizates. In the presence of the TESPT silane coupling agent, primary particles of silica of $50 \mathrm{~nm}$ and silica aggregates in the size of $100 \mathrm{~nm}$ are clearly visible in NR and DPNR vulcanizates. A bound rubber layer is observed on the primary particles of silica in the DPNR-TESPT vulcanizate. A better insight into filler-filler interaction is obtained from the micro- and nano-dispersion of silica by AFM and TEM measurements. 


\subsection{INTRODUCTION}

In recent years, high-dispersion silica has become the preferred alternative to carbon-black as reinforcing filler in tire tread compounds. Its use provides considerable improvement in rolling-resistance of passenger car tires. However, since silica is highly polar and hydrophilic, it is incompatible with apolar rubbers such as NR. Hence, mixing silica and rubber is difficult. A bi-functional silane coupling agent, such as bis(triethoxysilylpropyl) tetrasulfide (TESPT) is needed to improve the filler-to-rubber interaction on the nano-scale by creating chemical links between the silica surface and the rubber chains ${ }^{1,2}$.

The use of a silane like TESPT involves two main chemical reactions that need to take place at their appropriate time slots during rubber processing, namely the silica and silane reaction or silanization, and silane-rubber coupling. Since the silanization occurs insitu during mixing, it also correlates to dispersion of silica in the rubber matrix. A proper silanization of silica will results in a good dispersion of silica. Dispersion of silica can be categorized into macro-dispersion and micro-dispersion. Macro-dispersion is designated as dispersion of agglomerates with sizes of larger than $1 \mu \mathrm{m}$, while micro-dispersion refers to dispersion of aggregates of silica with a size smaller than $500 \mathrm{~nm}$. A dynamic equilibrium between silica particles, aggregates and agglomerates exists in rubber compounds as shown in Figure 6.1 ${ }^{3}$.

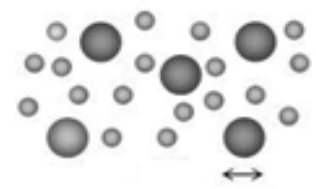

$10-50 \mathrm{~nm}$

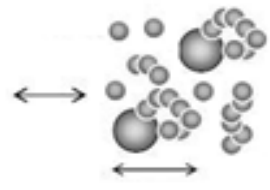

$100-500 \mathrm{~nm}$

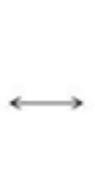

Aggregates

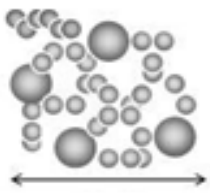

$1-40 \mu \mathrm{m}$

Primary particles

Figure 6.1 Primary particles, aggregates and agglomerates of silica.

Atomic Force Microscopy (AFM) is one of the foremost tools to study dispersion of fillers in polymer/rubber at nano-scale. AFM is a way of visualizing a surface using the forces between atoms ${ }^{4}$. The AFM probe is a cantilever with a sharp tip at its end that is used to scan the surface of the specimen. The principle of AFM is shown schematically in Figure 6.2. Most of the cantilevers are typically silicon or silicon nitride. The probe tip has a radius of curvature on the order of nanometers, normally $5-10 \mathrm{~nm}$. When the tip is brought into proximity of a specimen surface, forces between the tip and the specimen lead to a 
deflection of the cantilever, according to Hooke's law. The deflection is measured using a laser spot reflected from the top surface of the cantilever into an array of photodiodes. The principle of AFM is similar to that of a record player where a stylus is moving along a surface producing a variable signal dependent on the surface relief.

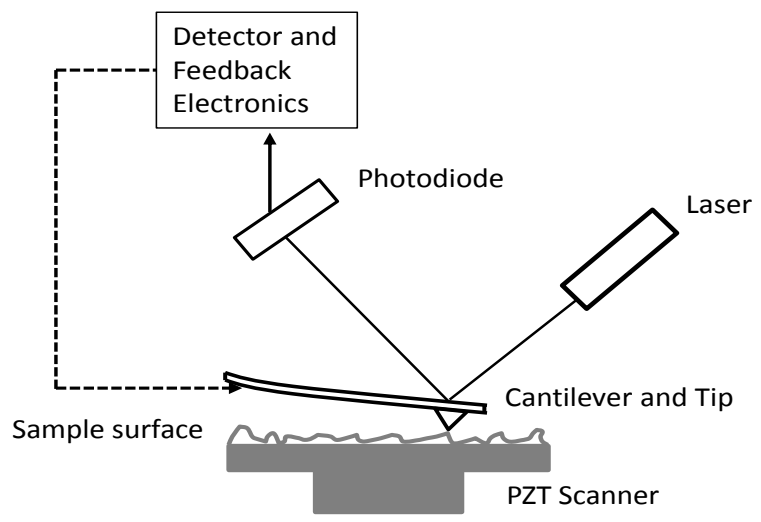

Figure 6.2 Principle of AFM.

There are 3 types of operating modes of AFM as shown in Figure 6.3. The first one is the contact mode where the force is measured by the reflection of the laser beam of the cantilever onto detector. A feedback system adjusts the $Z$ position of the cantilever to maintain constant force. The second mode is the tapping mode where the tip oscillates with an amplitude of several $\mathrm{nm}$. The typical frequency is $50-400 \mathrm{kHz}$. The tip touches the sample at the maximum amplitude. The feedback systems keep the amplitude constant throughout the scan. The third mode is the non-contact mode where it is basically the tapping mode but it remains $5-10 \mathrm{~nm}$ above the surface of the specimen and the tip registers differences in van der Waals attraction towards different moieties in the surface.

The effect of mixing dump temperature on micro-dispersion of silica in sSBR/BR blend compounds has been studied by Reuvekamp using AFM measurements ${ }^{5}$. In addition, AFM has also been used to visualize pre-vulcanization effects in the compounds: elliptical structures appears in the AFM phase image. Natchimuthi has investigated the effect of an epoxy resin on the dispersion characteristics of silica fillers in EPDM rubber using AFM ${ }^{6}$. Improved silica dispersion for EPDM-silica systems with epoxy resin was reported in terms of scanned height in the range of $0-100 \mathrm{~nm}$, as compared to the scanned height of a compound without resin which was found to be in the range of $0-700 \mathrm{~nm}$. Jeon et al. have successfully distinguished two different polymer domains in an unfilled rubber blend (natural 
rubber/synthetic rubber blend) using tapping mode $\mathrm{AFM}^{7}$. In the phase image, the harder polymer appears as the lighter phase in the view of image contrast. Using the same AFM technique, filler morphology in filled natural rubber and filled rubber blends was also examined by its shape as well as contrast.

(a) Contact mode

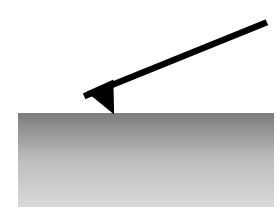

(b) Tapping mode

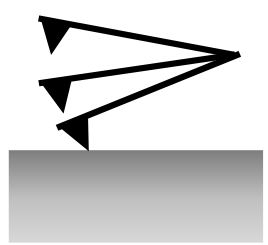

(c) Non-contact mode
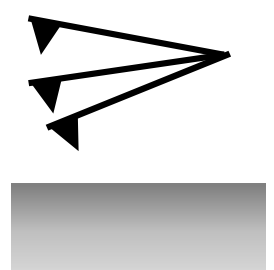

Figure 6.3 AFM operating modes: (a) Contact mode (b) Tapping mode (c) Non-contact mode.

In the present work, the macro- and micro-dispersion characteristics of silica in natural rubber compounds is studied using several morphological techniques. The macrodispersion is obtained using reflective optical microscopy and SEM. The micro-dispersion of silica is evaluated using non-contact tapping mode AFM and TEM. The influence of silane coupling agent, TESPT on morphological micro-dispersion is highlighted. NR is compared with purified NR from deproteinization (DPNR) as well as skim rubber for high protein content.

\subsection{EXPERIMENTAL}

\subsubsection{Materials}

Natural rubbers with various protein content were used, of which regular Natural Rubber (NR) SMR20 was taken as the reference, and compared to Deproteinized Natural Rubber (DPNR) as a low protein version, and Skim Rubber for the highest protein content. The ingredients and compound formulation were the same as what has been described in Chapter 4.

\subsubsection{Sample preparation}

The compounds were mixed and vulcanized according to the experimental section of Chapter 3. 


\section{Macro-dispersion measurements}

Macro-dispersion measurements were performed using a Dispergrader+ Tech Pro reflective optical light microscope. Vulcanized samples were cut with a razor blade and measured on three different spots using 100 times magnification. Using this method particle sizes of $3 \mu \mathrm{m}$ and larger can be detected.

\section{Scanning electron microscopy (SEM)}

Morphology characterization of tensile fractured surfaces of vulcanizates was carried out using a JSM-5000 NeoScope Benchtop SEM. The samples were sputter-coated with a thin layer of gold using a JEOL JFC-1300 automatic sputter coater.

\section{Sample preparation for AFM}

The vulcanizates (approx. $1 \times 5 \mathrm{~cm}$, along direction of grain) were extracted in refluxing acetone overnight. Each sample was wrapped in lens tissue $(5 \times 6 \mathrm{~cm})$. Several samples were extracted at once in the same solvent. After extraction was complete, the vulcanizates were allowed to dry to remove residual solvent. The surface of the extracted vulcanizate samples were cryo-microtomed at $-110^{\circ} \mathrm{C}$ using a glass knife. The sectioned samples were attached to the stub using Araldite glue.

\section{Atomic Force Microscopy (AFM)}

AFM measurements were conducted using an MFP-3D Stand Alone AFM (Asylum Research, Santa Barbara,CA,USA) at Tun Abdul Razak Research Centre, Brickendonbury, Hertford, UK. All measurements were done in non-contact tapping mode (dynamic, AC mode) at $k=2 \mathrm{~N} / \mathrm{m}$ and $\mathrm{f}_{0}=70 \mathrm{kHz}$. The $A F M$ images were processed using Argyle Light software by Asylum Research.

\section{Transmission Electron Microscopy (TEM)}

TEM analysis of the vulcanizates (not unextracted with acetone) was done using a Philips CM12 TEM operating at 80kV at the Tun Abdul Razak Research Centre (TARRC), Hertford, United Kingdom. An ultra-thin section of the sample was obtained by cryo-ultramicrotomy at $-110^{\circ} \mathrm{C}$ using glass and diamond knives. The microtome used was a PowerTome PC (RMC). 


\subsection{RESULTS}

\subsubsection{Macro-dispersion of silica-NR vulcanizates}

A comparison on macro-dispersion of silica among NR, DPNR and skim rubber vulcanizates with the means of reflective optical light microscope is presented in Figure 6.4. In this technique, the distribution of silica agglomerates with sizes of $3 \mu \mathrm{m}$ and larger can be detected. In addition, the white area percentage can be used as indication of silica macrodispersion in the vulcanizates. The silica agglomerates are easily observed in the vulcanizates with TESPT silane coupling agent. The details on the average size of agglomerates and percentage of white area for silica vulcanizates with TESPT is shown in Figure 6.5. The average sizes of silica agglomerates in NR and DPNR vulcanizates are comparable, which is about $13 \mu \mathrm{m}$. The skim rubber vulcanizate has smaller silica agglomerates with an average size of $10 \mu \mathrm{m}$. In comparison, NR and skim rubber vulcanizates without silane coupling agent have comparable agglomerate sizes, but the DPNR vulcanizate exhibits a larger size of silica agglomerates as shown in Table 6.1. The white area percentage for the DPNR vulcanizate without coupling agent is also higher than those of NR and skim rubber, which refers to poorer macro-dispersion of silica. The lowest white area percentage obtained for the skim rubber vulcanizate points to a positive effect of protein in the rubber to improve macro-dispersion of silica in absence of coupling agent.

NR
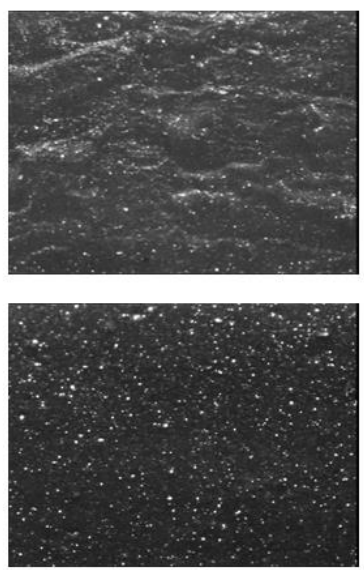

DPNR
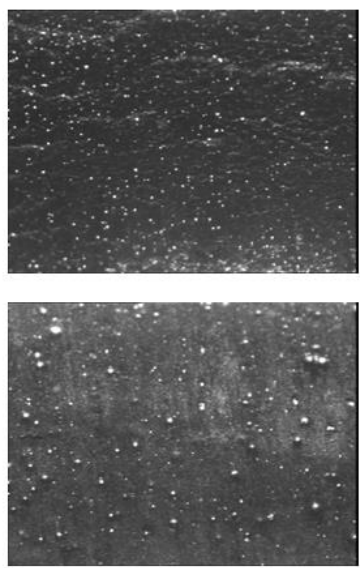

Skim Rubber
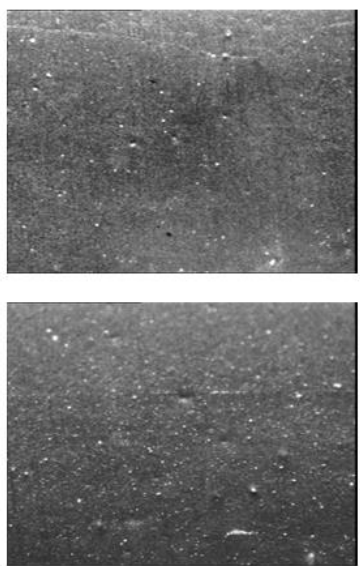

Figure 6.4. Macro-dispersion of silica in NR, DPNR and skim rubber vulcanizates in the absence and presence of TESPT coupling agent using reflective optical light microscopy.

The magnification was 100 times. 
A higher white area percentage is observed with increasing mixing dump temperature of the first stage mixing, which indicates a poorer macro-dispersion of silica in the vulcanizates at higher mixing temperature. However, the size of silica agglomerates remains constant with the variation in the mixing dump temperature. The results from reflective light microscopy indicates that the rubber types have a higher influence on the macro-dispersion of silica in the vulcanizates than the mixing dump temperature. Both NRsilica-TESPT and DPNR-silica-TESPT vulcanizates have comparable macro-dispersion, however skim rubber-silica-TESPT has slightly better macro-dispersion.

Table 6.1 Agglomerate size and white area \% of silica-filled vulcanizates without coupling agent

\begin{tabular}{lcc}
\hline Rubber type & Average agglomerate size, $\mu \mathrm{m}$ & White area, \% \\
\hline DPNR & 14.7 & 6.3 \\
NR & 11.6 & 3.3 \\
Skim Rubber & 11.6 & 0.4 \\
\hline
\end{tabular}

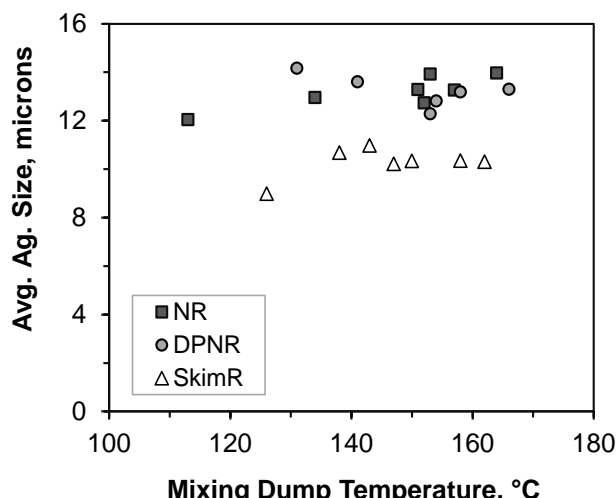

Mixing Dump Temperature, ${ }^{\circ} \mathrm{C}$

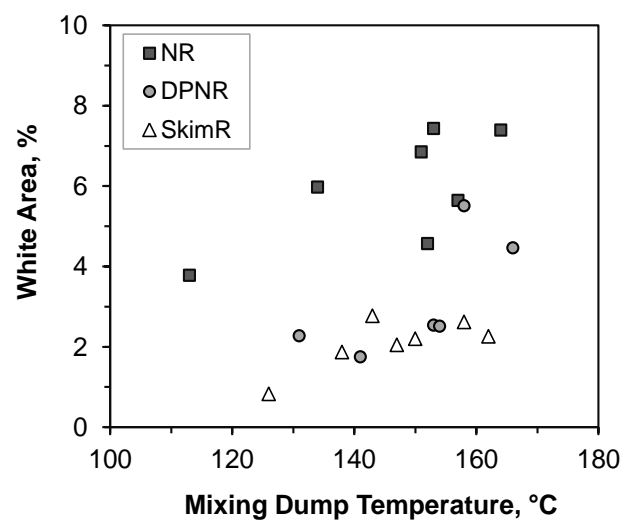

Figure 6.5 Comparison of average size of agglomerates and percentage of white area of silica vulcanizates with TESPT as a function of the $1^{\text {st }}$ stage mixing dump temperature.

\subsubsection{Morphology of silica macro-dispersion by SEM}

Morphology of macro-dispersion of silica-filled vulcanizates was further investigated by using SEM. In Figure 6.6, a comparison of tensile fracture surfaces between NR-silicaTESPT, DPNR-silica-TESPT and skim rubber-silica-TESPT vulcanizates is shown. The macro-dispersion (remaining agglomerates $>1 \mu \mathrm{m}$ ) of silica are observed to be comparable in all vulcanizates containing TESPT irrespective of the natural rubber types. 
A closer look at the surface morphology of silica vulcanizates in the absence and presence of TESPT is depicted in Figure 6.7. The filler agglomerates in the vulcanizates without silane are bigger than those with silane TESPT. In vulcanizates with TESPT, silica agglomerates with the size of $1 \mu \mathrm{m}$ and higher can be nicely distinguished and the distribution of the agglomerates is more homogeneous as compared to those without silane. A higher magnification than 5000 was also attempted but not successful to obtain clearer image with the bench top Joel SEM. In the image of the skim rubber vulcanizates without silane coupling agent the presence of foreign particles may be noticed which are assumed to be non-rubber constituents.

NR

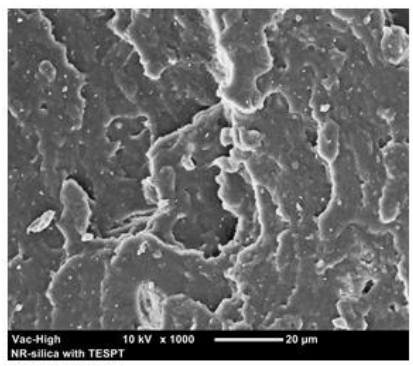

DPNR

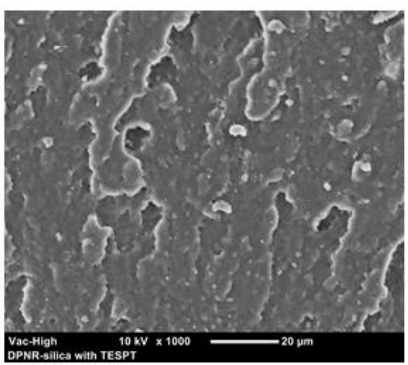

Skim Rubber

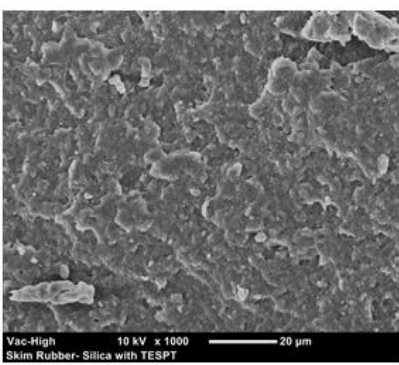

Figure 6.6 SEM micrographs of tensile fracture surfaces of silica-filled vulcanizates with TESPT coupling agent.
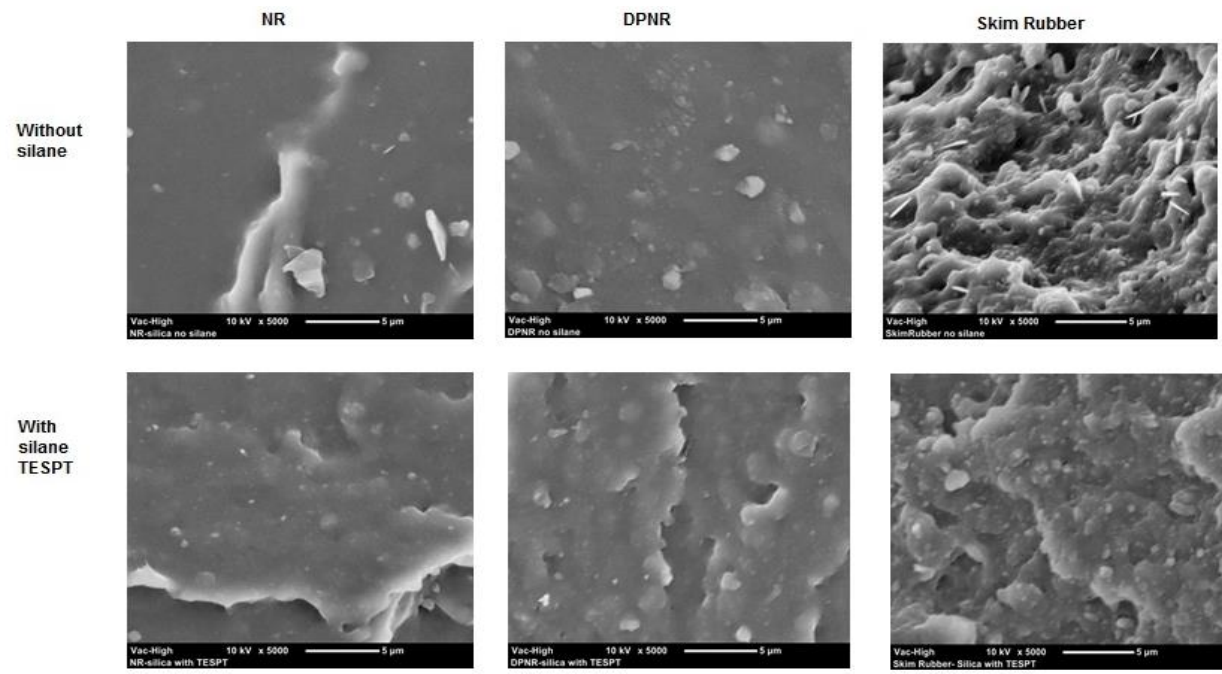

Figure 6.7 Comparison of SEM surface morphology of silica-filled vulcanizates in the absence and presence of TESPT coupling agent. 
Filler dispersion morphology affects the energy dissipation or hysteresis of silicafilled vulcanizates. The presence of fillers increases the hysteresis of rubber compound viscoelastically and hydrodynamically ${ }^{8}$. Therefore, the Mullins effect of NR-silica-TESPT vulcanizates is shown in Figure 6.8. The Mullins effect, or stress softening effect, is characterized by a reduction in stress when the filled-vulcanizate is extended for the second and subsequent time. Hysteresis can be measured from the area of the loop between the extension and retraction curves in a single tensile stress-strain cycle. Comparison of hysteresis of NR and DPNR vulcanizates with and without silane coupling agent is shown in Figure 6.9. The use of TESPT increases the hysteresis of silica-filled vulcanizates considerably due to strong filler-rubber coupling, but the differences between NR and DPNR are small if at all significant.

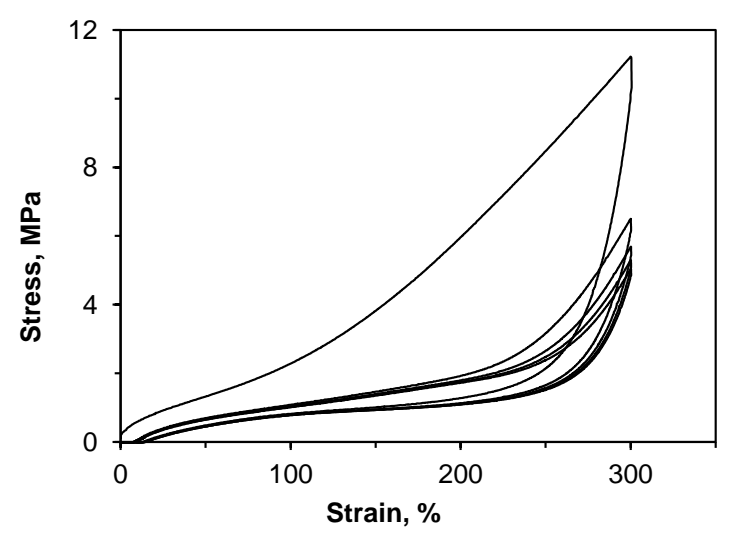

Figure 6.8 Mullins Effect of NR-silica-TESPT vulcanizate. 5 cycles till $300 \%$ strain.
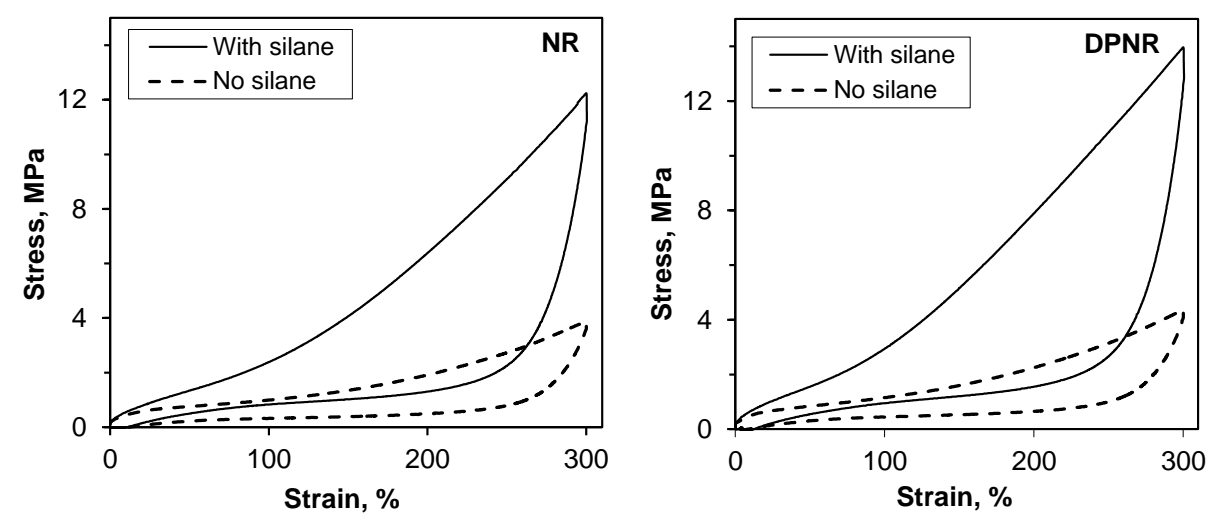

Figure 6.9 Comparison of hysteresis of NR and DPNR vulcanizates in the presence and absence of silane TESPT. 


\subsubsection{Micro-Dispersion of silica-NR vulcanizates by AFM}

Using non-contact tapping mode AFM, the height (topography), amplitude and phase images as well as 3D images of the samples can be obtained. Comparison of AFM images of NR-silica with DPNR-silica and skim rubber-silica vulcanizates in absence of TESPT silane coupling agent for a scan size of $5 \times 5 \mu \mathrm{m}$ are depicted in Figure 6.10. The left images are the height images and the right images are the phase images. In the height images, silica aggregates/agglomerates are shown in yellow, while rubber is shown in blue color. From the phase images, the silica aggregates/agglomerates can be differentiated based on the assumption that it has a higher stiffness compared to the rubber matrix ${ }^{9}$. Thus, in phase images silica aggregates/agglomerates are shown in blue, while rubber is shown in yellow color.

The structure of the silica aggregates forming agglomerates are readily distinguished from the height images: Figures 6.10 (a), (c), (e) based on the surface roughness at the same scanned heights in the range of $0-100 \mathrm{~nm}$. At scan size of $5 \times 5 \mu \mathrm{m}$ for the NR vulcanizate without coupling agent, the micro-dispersion of silica aggregates of the size 500nm and smaller is dominant, in addition to small agglomerates that are also present. The corresponding phase image in Figure 6.10(b) confirms the good microdispersion of silica in NR vulcanizate. The phase image of the DPNR vulcanizate without coupling agent shows comparable micro-dispersion of silica to that of NR, where silica aggregates of $500 \mathrm{~nm}$ and smaller are uniformly distributed. In the absence of a silane coupling agent, silica networking is clearly very strong in the skim rubber vulcanizates. In addition, the phase image for the skim rubber vulcanizate presents a poor micro-dispersion of silica, where a large number of aggregates close together forming agglomerates with size of $1 \mu \mathrm{m}$ and larger are detectable.

A comparison of AFM images of NR-silica with DPNR-silica and skim rubber-silica in presence of TESPT silane coupling agent is illustrated in Figure 6.11. From the height scan of $5 \times 5 \mu \mathrm{m}$ (Figures 6.11(a), (c) and (e)), micro-dispersion of silica is observed for the NR-silica-TESPT and DPNR-silica-TESPT vulcanizates, but only macro-dispersion is obtained for skim rubber-silica-TESPT vulcanizates, as agglomerates of the size $1 \mu \mathrm{m}$ are still visible. In addition, the NR vulcanizate has the lowest surface roughness. The size of the silica aggregates in NR appears to be smaller than in DPNR and skim rubber with TESPT from the height images. What is striking from the phase images for the vulcanizates with TESPT is the smaller aggregates size of silica as compared to the vulcanizates without silane. This indicates that a better micro-dispersion is obtained for silica vulcanizates with the use of TESPT. 

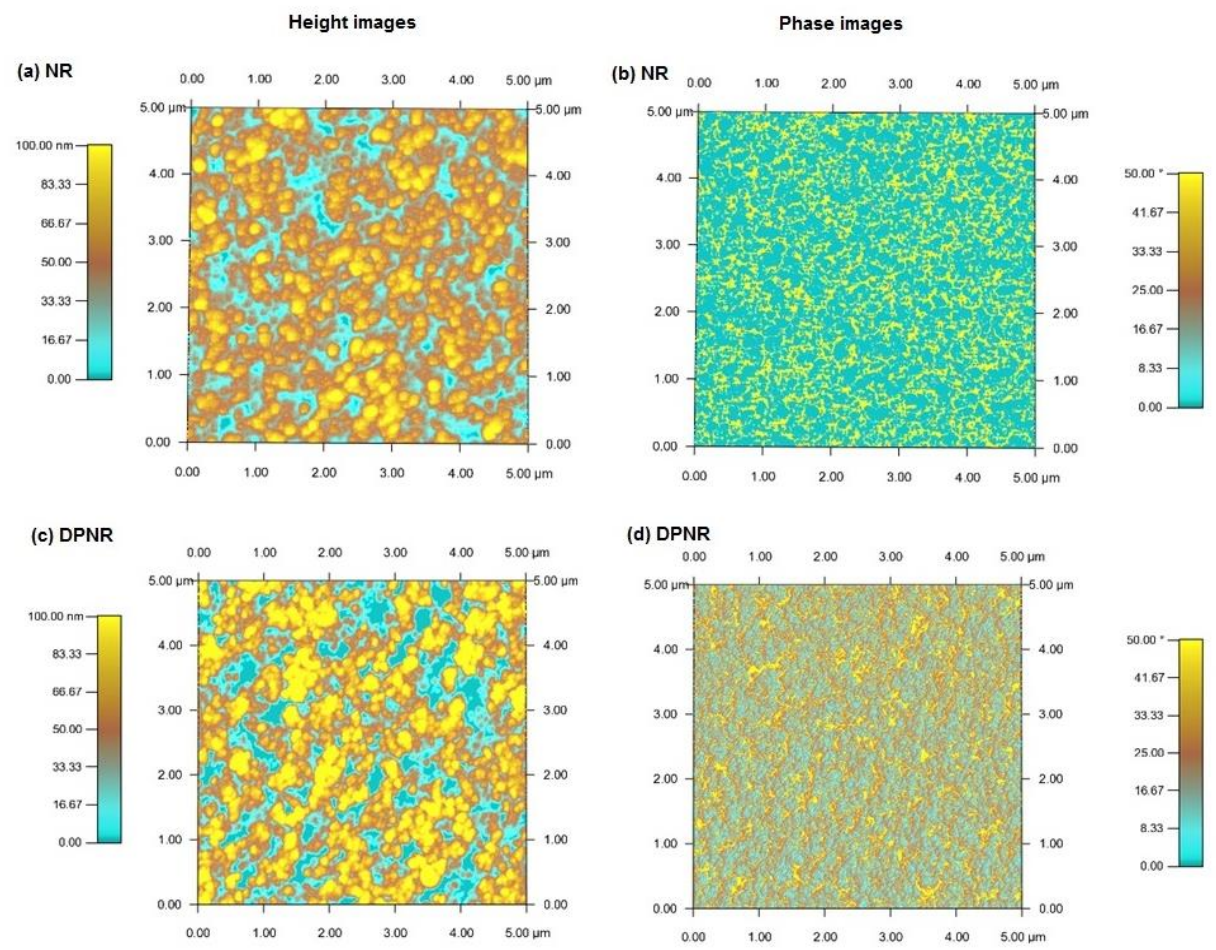

(e) Skim rubber

(f) Skim rubber
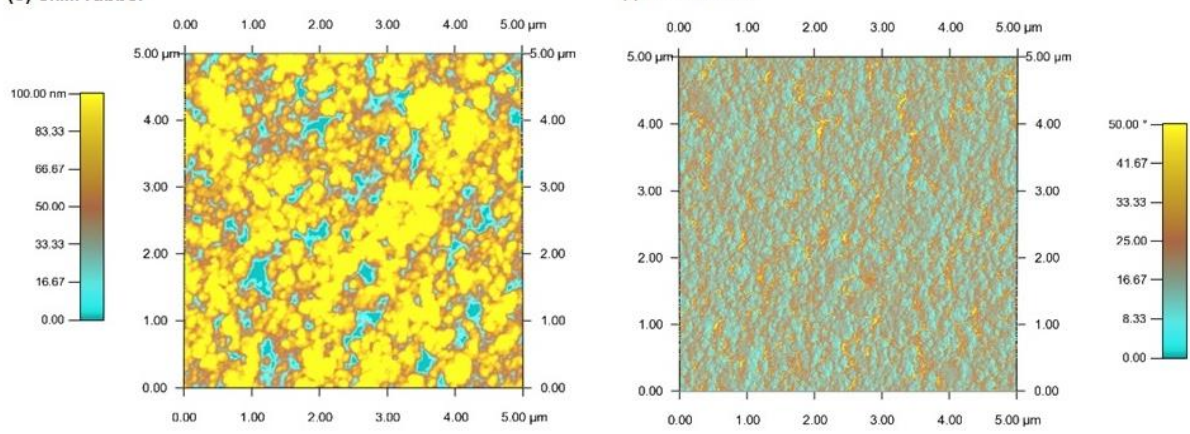

Figure 6.10 AFM images of silica vulcanizates in the absence of coupling agent with scan size of $5 \times 5 \mu \mathrm{m}$.

The phase images further reveal that the silica aggregates in NR and DPNR vulcanizates are approximately $100 \mathrm{~nm}$ and smaller. In addition, there are also aggregates of the size of $200 \mathrm{~nm}$ randomly visible, which is more obvious in the NR vulcanizate. 
Height images

(a) NR

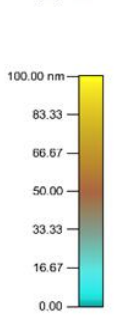

(c) DPNR

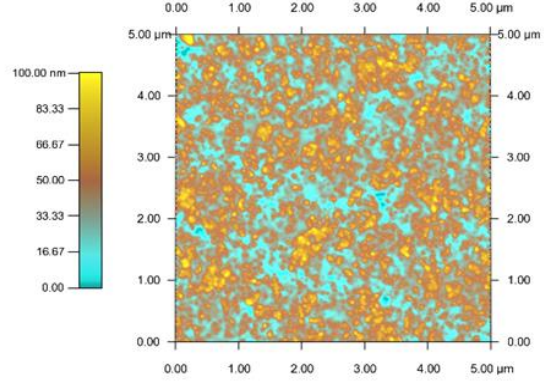

(e) Skim rubber

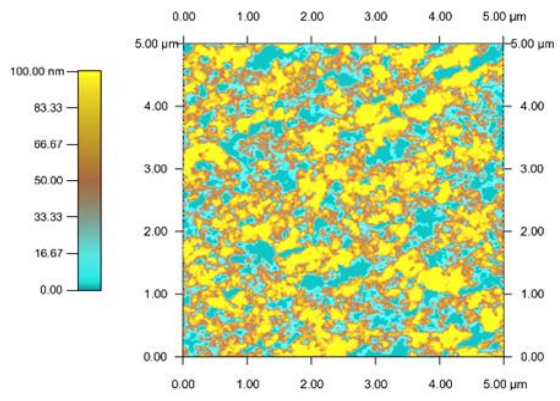

Phase images

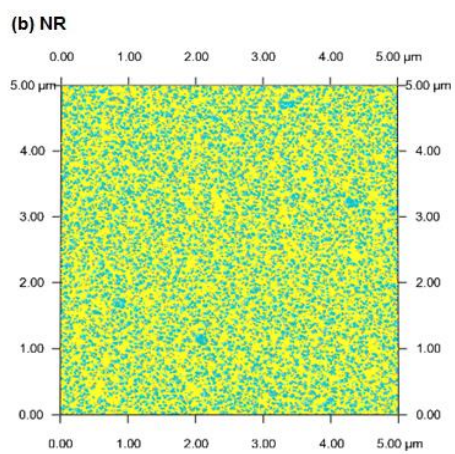

(d) DPNR

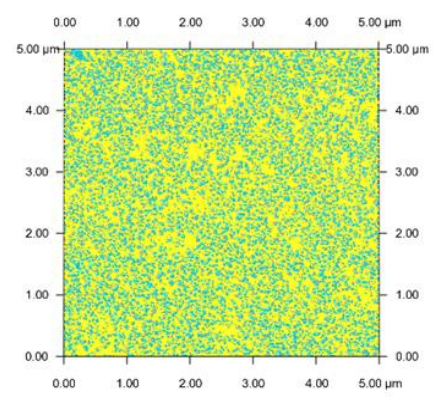

(f) Skim rubber

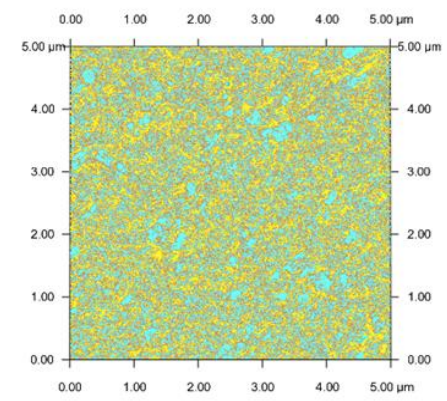

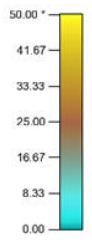

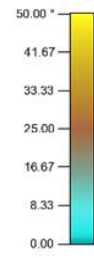

$\left.\begin{array}{l}50.00 * \\ 41.67- \\ 33.33- \\ 25.00- \\ 16.67- \\ 8.33- \\ 0.00\end{array}\right]$

Figure 6.11 AFM images of silica vulcanizates in the presence of silane coupling agent, TESPT with scan size of $5 \times 5 \mu \mathrm{m}$.

Overall, the silica aggregates in DPNR are somewhat more homogeneously dispersed than those in NR. It can be seen from the phase image of the skim rubber-silicaTESPT vulcanizate that it is a mixture of small aggregates of $100-200 \mathrm{~nm}$ and large aggregates of $200-500 \mathrm{~nm}$. This indicates poor micro-and macro-dispersion for skim rubber with TESPT as compared to NR and DPNR vulcanizates. 


\subsubsection{Nano-Dispersion of silica-NR vulcanizates by AFM}

The nano-dispersion of silica vulcanizates by AFM with even higher magnification in the absence of silane is illustrated in Figure 6.12. The size of the silica aggregates in DPNR and skim rubber vulcanizates without coupling agent is bigger than in the NR vulcanizate as seen from the height image at $1000 \times 1000 \mathrm{~nm}$. The phase image of NR-silica without silane in Figure 6.12(b) shows the silica aggregates of 100nm size and smaller as dispersed in the matrix. The size of the silica aggregates in the DPNR vulcanizate is almost comparable to that in NR, although they seem to be closer together. In contrast, the silica aggregates are clearly agglomerated in the phase image of skim rubber vulcanizates, where the silica aggregates size is $200 \mathrm{~nm}$ and bigger as shown in Figure 6.12(f). Our earlier work in Chapter 4 has shown that with the high protein content, skim rubber without silane exhibits a lower Payne effect than NR and DPNR. It is quite surprising that the aggregate size in skim rubber as seen from height image is more than $500 \mathrm{~nm}$ and clearly bigger than in NR and DPNR. Even though the silica-silica network is disrupted by proteins, the silica and protein interactions eventually form a silica-protein network at equilibrium and this results in silicaprotein agglomerates. The corresponding phase image shows the aggregates of $100 \mathrm{~nm}$ and bigger are glued together forming an aggregate structure, which indicates a poor microdispersion of silica in the skim rubber vulcanizate without silane.

In Figure 6.13, the height images show an improved nano-dispersion of silica in NR and DPNR vulcanizates with the use of TESPT as compared to without coupling agent. The primary particles of silica in the size of $50 \mathrm{~nm}$ are clearly visible in both NR and DPNR in addition to silica aggregates of approximately $100 \mathrm{~nm}$. The difference between NR and DPNR can be observed from the phase image. The distances between the silica aggregates of size $50-100 \mathrm{~nm}$ are clearly visible in the NR-silica-TESPT vulcanizate. However, in DPNRsilica-TESPT, the distance between the aggregates looks a little smaller, indicating a somewhat better nano-dispersion. Besides, there is an intermediate region between the silica and rubber phases. A study by Nakajima and Nishi by AFM analysis on carbon black filled-NR vulcanizates showed the existence of an intermediate phase surrounding the carbon black region whose Young's modulus was stiffer than the rubbery region but softer than the filler region ${ }^{10}$. Bielinski et al. have also shown a filler-matrix interphase from AFM images, ascribed to a bound rubber layer ${ }^{11}$. However, the filler-rubber interphase is found to be different for carbon black and silica mixes ${ }^{12}$. The interpretation of this intermediate region is still subject to debate as some blame it on a shadowing effect of AFM. 
Height images

(a) NR
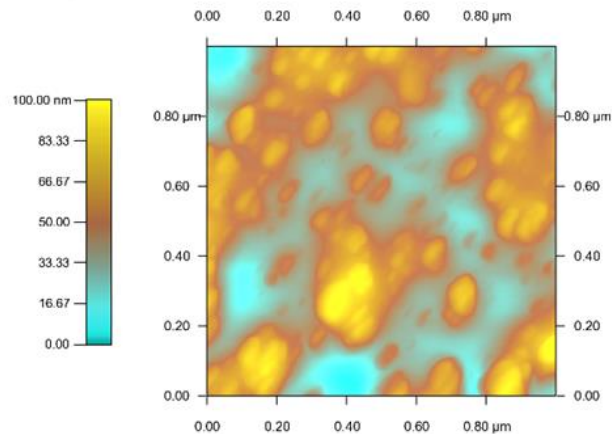

(c) DPNR

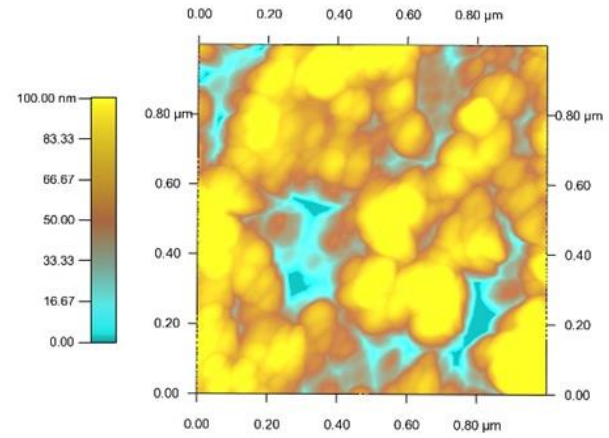

(e) Skim rubber

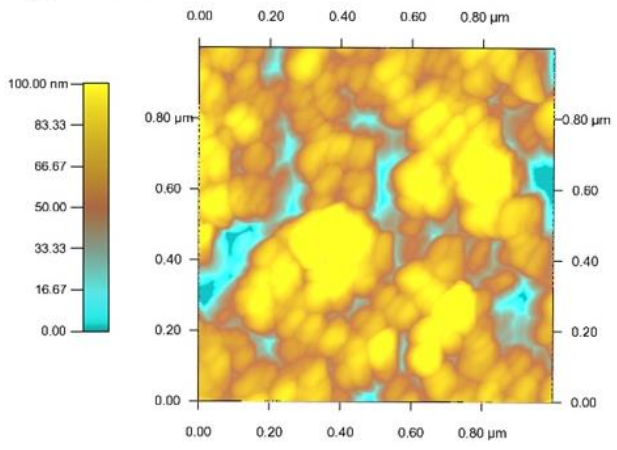

(b) NR

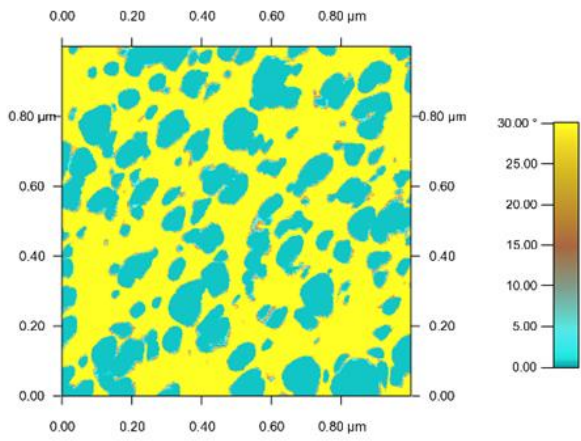

(d) DPNR
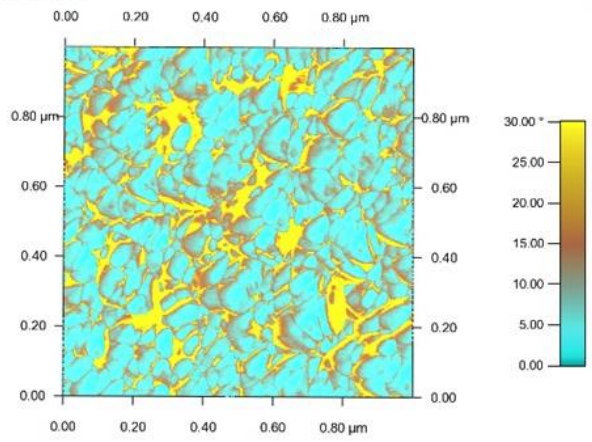

(f) Skim rubber

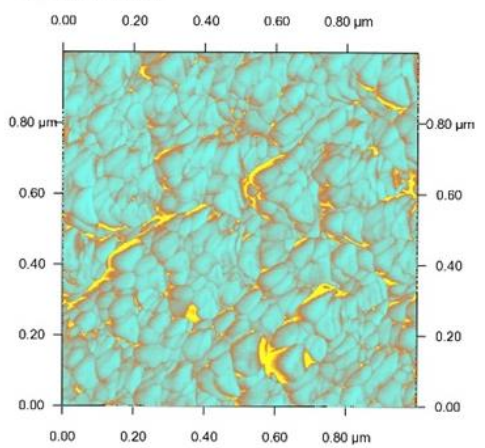

Figure 6.12 AFM images of silica vulcanizates in the absence of coupling agent with scan size of $1000 \times 1000 \mathrm{~nm}$. 
Height images

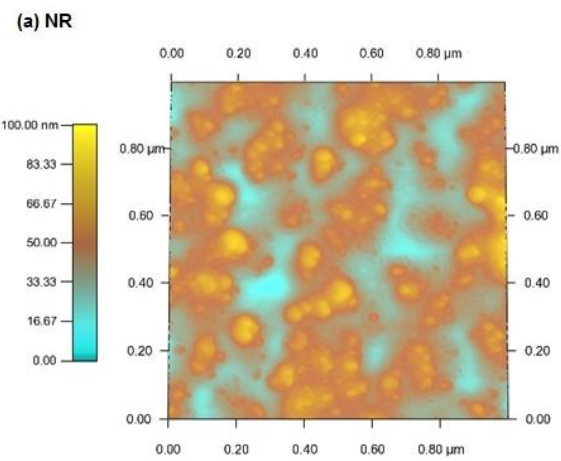

(c) DPNR

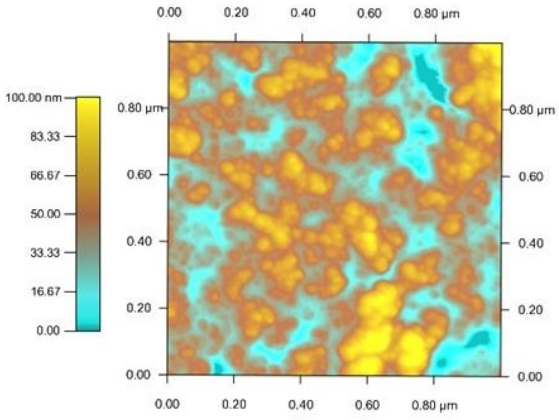

(e) Skim rubber

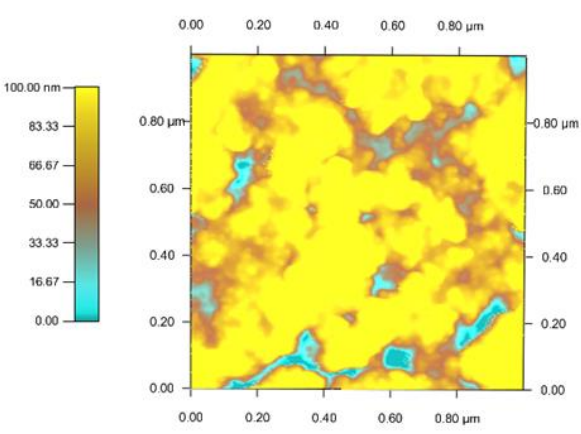

Phase images

(b) NR

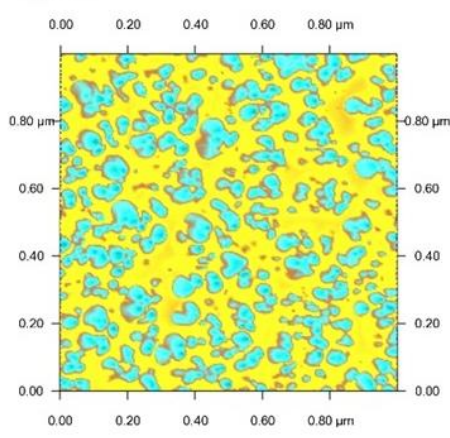

(d) DPNR

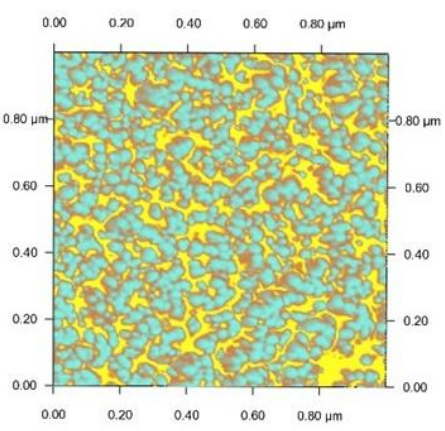

(f) Skim rubber

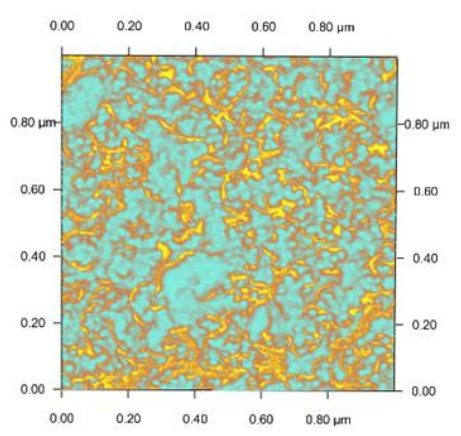

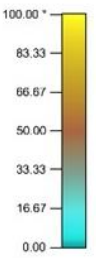
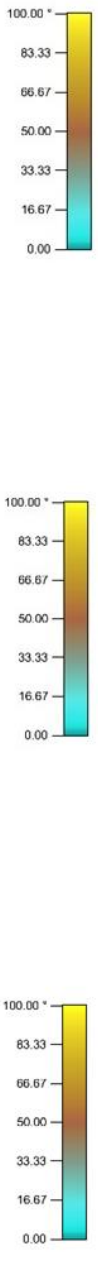

Figure 6.13 AFM images of silica vulcanizates in the presence of silane coupling agent, TESPT with scan size of $1000 \times 1000 \mathrm{~nm}$.

In Figure 6.13(d), the intermediate region is clearly observed surrounding the silica aggregates, rather than only on one side. This suggests that there is a bound rubber phase covering the silica aggregates in DPNR, which demonstrates that there is more rubber-to- 
filler interaction in the presence of silane TESPT. This may be related to better silanization in DPNR as low amounts of protein are present in the rubber, and results in lower filler-filler interaction. For the skim rubber-silica-TESPT vulcanizate, only large agglomerates of silica are visible from the height image, which is similar to the one without coupling agent, which indicates that a poor micro-dispersion and no nano-dispersion is obtained. In addition, the phase image shows a strong silica network with big aggregates to agglomerate together resulting from silica-protein interaction. However, the intermediate region is also observed for the skim rubber vulcanizate, which indicates that the silanization occurs only to limited extent. The AFM morphology of the skim rubber-TESPT-silica vulcanizate confirms the earlier finding that the high amount of proteins present in the rubber can hinder the silanization of silica and result in silica not well dispersed to the micro-level.

\subsubsection{Nano-Dispersion of silica-NR vulcanizates by TEM}

TEM images showing the dispersion of silica particles in vulcanizates with TESPT are presented in Figure 6.14. The TEM image reveals that the silica is mainly dispersed to primary particles due to increase hydrophobation of silica surface by TESPT. The average size of the primary particle of silica is about $20 \mathrm{~nm}$. In comparison, the DPNR-silica-TESPT vulcanizate exhibits better nano-dispersion than the NR-silica-TESPT vulcanizate. The primary particles and aggregates of silica are homogeneously distributed in the NR and DPNR vulcanizates with TESPT. However, for skim rubber vulcanizate with TESPT, the silica aggregates appear to be separated in addition to randomly distributed silica particles.

A further investigation on skim rubber vulcanizates is attempted to clarify the silica and proteins interaction. Proteins are not visible in the TEM unless it is stained. Uranyl acetate stains both negatively and positively charged side-chains on the protein molecules ${ }^{13}$ and with this method the proteins can be visualized. The TEM images of skim rubber vulcanizates stained with uranyl acetate are shown in Figure 6.15. In both vulcanizates, with and without silane, globular features stained by uranyl acetate are clearly visible. This indicates the proteins in skim rubber are present as globules in the size of $100-300 \mathrm{~nm}$, which is equivalent to the size of silica aggregates. In the absence of silane, the silica is observed to settle around the globules and also inside the globular proteins. In the presence of silane, the silica is much less surrounding the globular proteins and very little is penetrated into the proteins globules. The presence of these globular proteins explains the separation of silica aggregates in the skim rubber vulcanizate in Figure 6.14(c), which results in a poor micro-dispersion of the silica. It also confirms the earlier discussion that the 
silica-protein interactions form a silica-protein network which results in big agglomerates observed in the AFM images of the skim rubber vulcanizates.
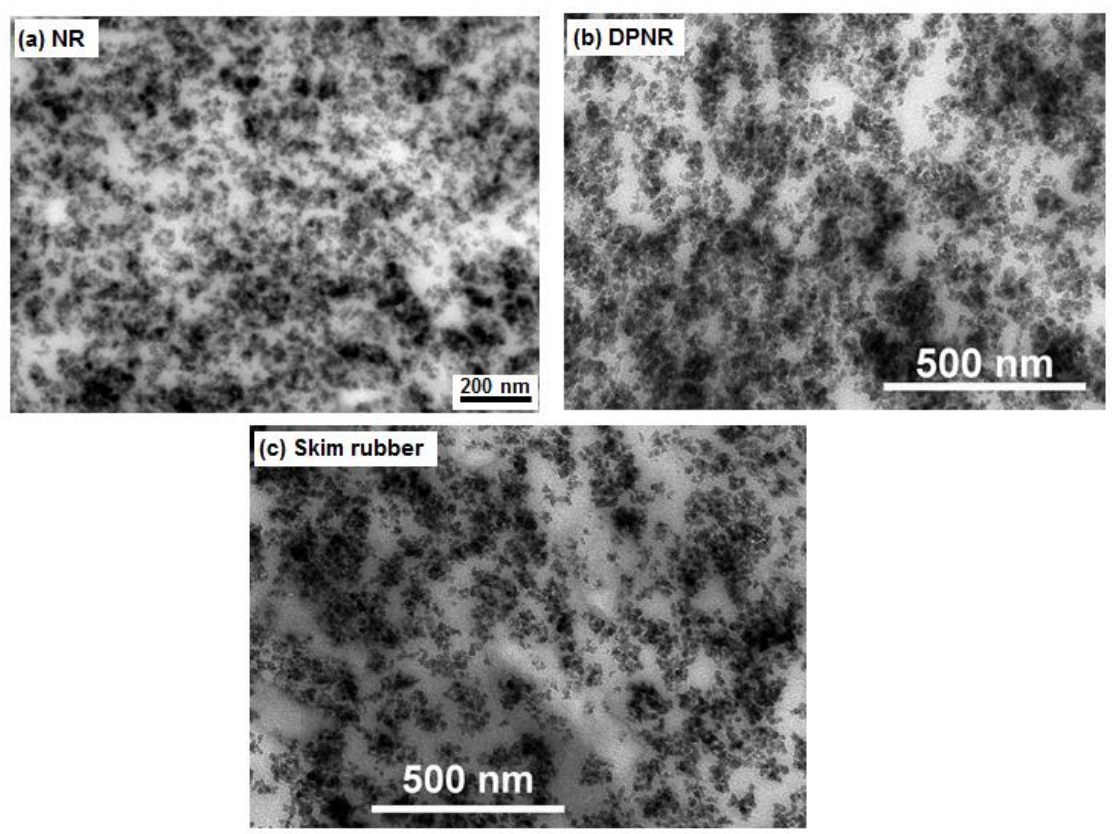

Figure 6.14 Comparison of TEM images of silica vulcanizates in the presence of silane coupling agent, TESPT.
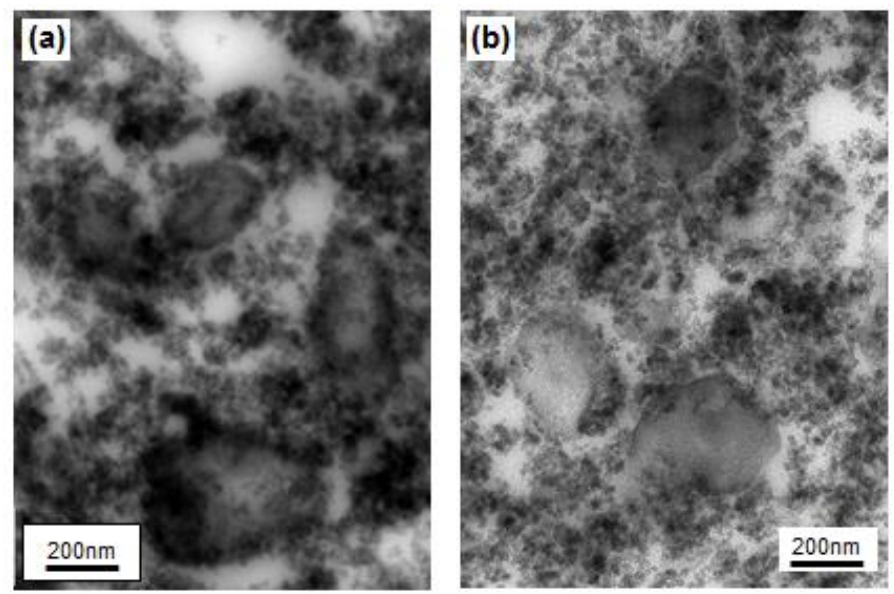

Figure 6.15 Comparison of TEM images of skim rubber-silica vulcanizates stained with uranyl acetate: (a) without silane (b) with silane coupling agent, TESPT. 


\subsubsection{Payne Effect of silica-NR vulcanizates}

A comparison of the Payne effect of silica-filled NR, DPNR, and skim rubber vulcanizates is shown in Figure 6.16. In the absence of silane coupling agent, the Payne effects of NR and DPNR are almost comparable. This is in agreement with the results of micro-dispersion. The Payne effects of the NR and DPNR vulcanizates with silane is considerably lower than those without silane. This again correlates well with a good micro- and nano-dispersion of NR- and DPNR-silica-TESPT vulcanizates, which is confirmed by the AFM phase images in Figures 6.11 and 6.13. The lower Payne effect which results from reduced filler-filler interaction in the DPNR vulcanizate as compared to NR indicates that there is more coupling between TESPT and silica with less protein present or purified NR. This is also in agreement with the intermediate region surrounding the silica aggregates in DPNR-silica-TESPT aggregates, which is ascribed to the bound rubber layer as discussed earlier.

The skim rubber without silane has the lowest Payne effect, which indicates lower filler-filler interaction and the effect of proteins in the hydrophobation of silica. This is rather puzzling as this only relates to the good macro-dispersion of silica, as the skim rubber without silane has poor micro- and nano-dispersion from AFM analysis. In the presence of silane coupling agent, TESPT, the skim rubber vulcanizate has the highest Payne effect, which demonstrates the opposite effect when silane is present in the skim rubber compound. This clearly points to a competitive effect between protein and silane in the hydrophobation of the silica surface. The high Payne effect value supports the poor microand macro-dispersion obtained for the skim rubber vulcanizate with TESPT.

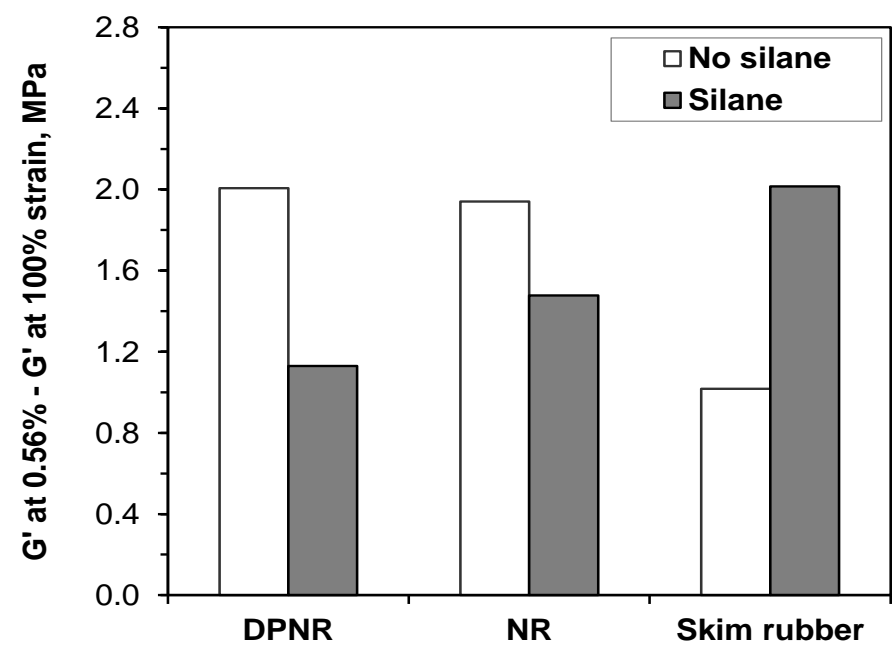

Figure 6.16 Payne effect of silica vulcanizates with different natural rubber types. 


\subsection{Discussion}

A good micro-dispersion of silica in the rubber vulcanizates will determines the final properties. With different morphological techniques, the macro-, micro- and nano-dispersion of silica in natural rubber has been illustrated. The different levels of silica dispersion are schematically illustrated in Figure 6.17. At nano-dispersion level, a bound rubber layer is observed on primary particles of silica.

Evaluation of macro-dispersion of filler by reflective light microscopy and SEM is not sufficient to reveal differences between degree of silica dispersion in NR, DPNR and skim rubber vulcanizates. The average agglomerate sizes of silica in NR and DPNR are comparable, as seen from the reflective light microscopy, which is in the range of $12-14 \mu \mathrm{m}$. Meanwhile, the average agglomerate size of silica in the skim rubber vulcanizate is only slightly smaller: about $10 \mu \mathrm{m}$. The morphology of macro-dispersion of silica by SEM is comparable in all vulcanizates irrespective of the natural rubber types.

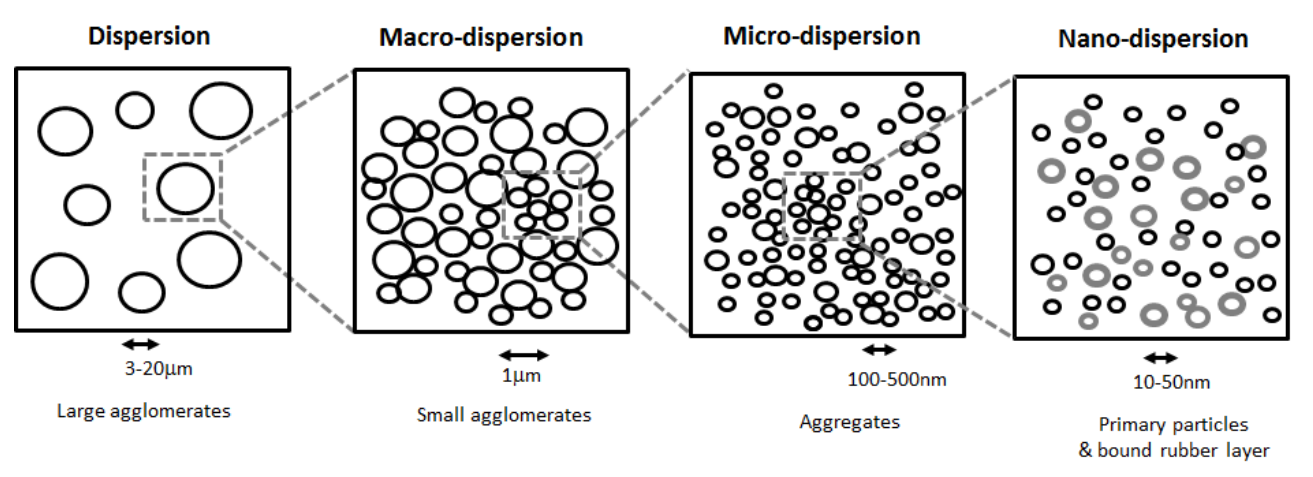

Figure 6.17 Schematic illustration showing the different levels of dispersion from macro, to micro and nano-dispersion of fillers.

In the absence of the silane coupling agent, the AFM phase image reveals that both NR and DPNR-vulcanizates have the silica dispersed to the micro level. This correlates with the results from the Payne effect. For skim rubber vulcanizate without silane, the height and phase images from AFM analysis show strong silica-networking. This is opposite to the lower Payne effect observed. Micro-dispersion of silica is observed in all vulcanizates with TESPT from the AFM phase images, where aggregates sizes of $500 \mathrm{~nm}$ are dominant.

The results have shown that the use of coupling agent TESPT enhances the dispersion of silica into aggregates and even into primary particles, which results in an increase of filler-to-rubber interactions. This is confirmed by the good nano-dispersion of 
silica in the NR-silica-TESPT and DPNR-silica-TESPT vulcanizates from the TEM- and AFM-phase images where primary particles of silica of $20-50 \mathrm{~nm}$ are uniformly dispersed. The skim rubber-silica-TESPT has the worst micro- and nano-dispersion which again points to the competitive effect of silane and protein in hydrophobation of the silica surface. The TEM image of the skim rubber vulcanizate reveals that the proteins exist in globular structures which have the same size as the silica aggregates. It may be postulated, that the globular proteins have a tendency for preferential silane adsorption on their surface which results in a reduced efficiency of the silane to cover the surface of silica.

The results have shown that the different morphological techniques do not always give consistent information on the dispersion of filler in the rubber. The AFM analysis on micro- and nano-dispersion of silica does correlate the best with the Payne effect of the vulcanizates. This only applies to NR and DPNR vulcanizates. Skim rubber with such a high protein content, and also its production method - acid coagulation-apparently should be treated as totally different material. A proper comparison of skim rubber with NR and DPNR does not really work. All in all, even with the use of highly dispersible silica in the compound, the good micro-and nano-dispersion of silica is influenced by the rubber types, silane coupling agent and compound preparation.

\subsection{CONCLUSIONS}

A comparative morphological study on macro-, micro- and nano-dispersion of silica between Natural Rubber (NR), Deproteinized Natural Rubber (DPNR) and skim rubber vulcanizates has been presented, as executed with different morphological techniques. The micro- and nano-dispersion of silica observed by means of tapping mode Atomic Force Microscopy (AFM) gives the best insight into filler-filler and polymer-filler interactions. In the absence of a silane coupling agent, silica networking is clearly very strong in skim rubber as compared to the pure NR vulcanizate. In the presence of TESPT silane coupling agent, primary particles of silica of $50 \mathrm{~nm}$ and silica aggregates in the size of $100 \mathrm{~nm}$ are clearly visible in NR and DPNR vulcanizates. A bound rubber layer is also observed on the primary particles of silica in the DPNR-TESPT vulcanizate. In addition, the silica aggregates in DPNR are slightly more homogeneously dispersed than those in NR, indicating a good microdispersion of silica in DPNR-TESPT, which correlates well with the lower Payne effect of the vulcanizate. The interaction between silica and proteins in skim rubber vulcanizate is further illustrated by the TEM nano-dispersion, where large protein globules of size approximately $300 \mathrm{~nm}$ block a good nano-dispersion of the silica aggregates; possibly also by preferred adsorption of the coupling agent TESPT. It is clear that the micro- and nano-dispersion is 
strongly influenced by the different qualities of the natural rubber types, the presence of a silane coupling agent and the compound preparations.

\subsection{REFERENCES}

1. W. Meon, A. Blume, H-D. Luginsland, and S.Uhrlandt, in Rubber Compounding: Chemistry and Applications, (Ed. B. Rodgers), Marcel Dekker Inc., New York, Ch.7 (2004).

2. J.W.M. Noordermeer and W.K. Dierkes, in Rubber Technologist's Handbook, Vol.2, (Eds.: J. White, S.K. De and K. Naskar), Smithers Rapra Technology, Shawbury, Shrewsbury, Shropshire, UK, Ch.3 (2008).

3. Y. Li, M.J. Wang, T. Zhang, F.Zhand and X. Fu, Rubber Chem. Technol., 67, 693 (1994).

4. B. Cappella and G. Dietler, Surface Science Reports, 34, 1-104 (1999).

5. L.A.E.M. Reuvekamp, PhD thesis, University of Twente, Enschede, the Netherlands (2003).

6. N. Natchimuthu, Rubber Chem. Technol., 83, 123 (2010).

7. I.H. Jeon, H. Kim and S.G. Kim, Rubber Chem. Technol., 76, 1 (2002).

8. A.R. Payne, J. Polymer Sci.:Symposia. 48, 169 (1974).

9. D.R-V Haeringen, H. Schönherr, G.J. Vansco, L. van der Does, J.W.M. Noordermeer and P.J. P. Janssen, Rubber Chem. Technol., 72, 862 (1999).

10. K. Nakajima and T. Nishi, in Current Topics in Elastomers Research, (Ed. A. K. Bhowmick), CRC Press, New York, Ch. 21 (2008).

11. D. Bielinski, L. Slusarski, O. Dobrowolski, E. Dryzek, Kautsch. Gummi Kunstst., 57, 579 (2004).

12. D. Bielinski, L. Slusarski, O. Dobrowolski, E. Dryzek, Kautsch. Gummi Kunstst., 58, 239 (2005).

13. M.A. Hayat, Principles and Techniques of Electron Microscopy Biological Applications, Third Edition, Macmillan Press, London (1989). 
Chapter 6 


\title{
Chapter 7
}

\section{Mechanistic Study into the Effect of Proteins on Hydrophobization of Silica Surfaces}

\begin{abstract}
Adsorption of proteins on silica surfaces is driven by many factors. The interactions of proteins and silica are able to disrupt the silica-silica networking in natural rubber (NR) compounds. When a silane coupling agent is used in a silica compound, both silane and proteins compete for the silica surface and this leads to the silanization process being hindered, which results in low silica reinforcement. Proteins from NR serum are used in an in-rubber study to investigate the filler-filler and filler-to-rubber interactions in both NR and synthetic polyisoprene (IR) systems. The mechanism of proteins in hydrophobization of silica surfaces is investigated through the interaction of flat glass surfaces to mimic the surface of silica, with the NR serum protein. Silica surfaces treated with silane and proteins are evaluated using contact angle measurements. A comparison is made between treated glass surface and treated silica powder. The results from the contact angle measurements show a strong hydrophobization effect of the NR serum protein on silica surfaces as compared to the silane.
\end{abstract}




\subsection{INTRODUCTION}

Adsorption of proteins on a solid surface is a common but very complicated phenomenon ${ }^{1,2}$. The interaction of proteins with solid surfaces such as silica has been subject of investigation in various fields such as biotechnology, medicine, food processing and environmental science ${ }^{3,4}$. Protein adsorption is a very complex process which is driven by many parameters, among others are $\mathrm{pH}$, temperature, the ionic strength, protein concentration, protein stability, the properties of the protein and the nature of the solid surface itself ${ }^{5}$.

In silica reinforced natural rubber (NR) compounds, proteins and coupling agent show a complicated antagonistic effect ${ }^{6}$. The effect of proteins is most pronounced when no silane is used in the NR-silica compounds ${ }^{7}$. When high amounts of proteins are present in $\mathrm{NR}$, the interactions between proteins and silica are able to disrupt silica-silica networking. When a silane is used in the silica compounds, both silane and proteins compete for silica surface and this leads to the silanization process being hindered, which results in low silica reinforcement.

Although proteins adsorb on solid surfaces through various interactions, the important forces in particular are hydrophobic/hydrophilic and electrostatic interactions ${ }^{1}$. Krisdhasima et al. have shown that an increase in hydrophobicity of a chemically modified silicon surface led to an increase in the amount of protein ( $\beta$-lactoglobulin) adsorbed ${ }^{8}$. Buijs and Hlady studied the adsorption of recombinant human growth hormone on modified silica surfaces, and concluded that hydrophobic interaction significantly contributes to strong protein and surface interaction, which is accompanied by a less compact protein conformation $^{9}$. Tarasevich and Monakhova have shown that the carbonyl-groups of albumin molecules interact with vicinal hydroxyl groups, and amide groups with individual hydroxyl groups of silica ${ }^{10}$. The geminal hydroxyl groups of the silica surface behave as single adsorption sites with respect to albumin ${ }^{10}$. Gunko et al. described protein molecules adsorbed on fumed silica aggregates by hydrogen bonds with $\equiv \mathrm{SiOH}$ or $\equiv \mathrm{SiO}^{-}$groups by amino groups, eg. $\mathrm{H}+\mathrm{NR}$ from zwitterion fragments ${ }^{11}$. The protein adsorption rate decreases with increasing molecular weight of the proteins ${ }^{11}$. Increase in ionic strength reduces the amount of protein being adsorbed on silica and complete removal of protein can be achieved at high ionic strength ${ }^{12}$. On the other hand, increase in the concentration of surface silanols with growing size of primary particles of silica enhances protein adsorption in $\mathrm{mg} \cdot \mathrm{m}^{-2}$ of the silica surface ${ }^{13}$. Rezwan et al. have shown that adsorption to the hydrophilic silica surface increases with a protein of opposite charge, compared to proteins of the same charge where only low amounts are adsorbed ${ }^{14}$. 
The aim of the present chapter is to gain more insight into the mechanism of proteins and silica interaction. The kinetics of silanization of silica in natural rubber (NR) and synthetic polyisoprene (IR) systems are first demonstrated. The proteins from NR serum are used in the in-rubber study, to investigate the filler-filler and filler-to-rubber interactions in both NR and IR systems. The mechanism of proteins in hydrophobization of silica surfaces is then further investigated from the interaction of flat glass surfaces, to mimic the surface of silica, with NR serum proteins. Comparison is made between a treated glass surface and treated silica powder. Silica surfaces treated with coupling agent and proteins are evaluated using contact angle measurements.

\subsection{EXPERIMENTAL}

\subsubsection{Materials}

Polyisoprene (IR2200) was supplied by Nipol Zeon as described in Chapter 3. Standard Malaysian Natural Rubber (SMR 20) was provided by the Malaysian Rubber Board (MRB). Silica Ultrasil 7005 and silane coupling agent, bis-(triethoxysilylpropyl)- tetrasulfide (TESPT) were supplied by Evonik. Natural Rubber (NR) serum protein powder was provided by the MRB. The typical properties of NR serum protein powder are listed in Table 7.1. The method of extracting the NR serum protein is described elsewhere ${ }^{15}$. Treated distillate aromatic extract (TDAE) oil was from Hansen \& Rosenthal. Zinc oxide, stearic acid and sulfur were from Sigma Aldrich. Antioxidant TMQ, N-cyclohexyl-2-benzothiazyl sulfenamide (CBS) and diphenyl guanidine (DPG) were from Flexsys. All ingredients were used as received.

Table 7.1 Natural Rubber Serum Protein Powder

\begin{tabular}{ll}
\hline \multicolumn{1}{c}{ Properties } \\
\hline Total protein content & $95-98 \%$ \\
Average particle size & $40-60 \mu \mathrm{m}$ \\
Color/powder form & White, free flowing \\
Main amino acids & Cysteine, lysine, tyrosine \\
\hline
\end{tabular}

\subsubsection{Compounding}

The compound formulations are shown in Table 7.2. All ingredients except the curatives were mixed in an internal mixer, Brabender Plasticoder 350S lab station. The mixing was done at $60 \mathrm{rpm}$ rotor speed, 0.7 fill factor, 14 minutes mixing time and $150^{\circ} \mathrm{C}$ dump temperature. After 24 hours, the curatives were added to the masterbatches on a two-roll mill. 
Table 7.2 Compound Formulations

\begin{tabular}{llllll}
\hline Ingredients & I & II & III & IV & V \\
\hline IR (Nipol IR2200) & 100 & - & 100 & - & 100 \\
NR (SMR20) & - & 100 & - & 100 & - \\
Silica Ultrasil 7005 & 55 & 55 & 55 & 55 & 55 \\
NR Serum Protein & - & 12 & - & 12 & 12 \\
TESPT & - & - & - & - & 5 \\
Zinc Oxide & 2.5 & 2.5 & 2.5 & 2.5 & 2.5 \\
Stearic acid & 1 & 1 & 1 & 1 & 1 \\
TDAE oil & 8 & 8 & 8 & 8 & 8 \\
TMQ & 2 & 2 & 2 & 2 & 2 \\
Sulfur & 1.4 & 1.4 & 1.4 & 1.4 & 1.4 \\
CBS & 1.7 & 1.7 & 1.7 & 1.7 & 1.7 \\
DPG & 2 & 2 & 2 & 2 & 2 \\
\hline
\end{tabular}

\subsubsection{Surface modification of silica powder and glass slides}

\subsubsection{Silica powder treated with silane}

About $0.45 \mathrm{~g}$ of TESPT was dissolved in $50 \mathrm{ml}$ of ethanol. The solution was stirred for 30 minutes. $5 \mathrm{~g}$ of silica was slowly added to the solution until all the silica was dispersed in the ethanol. The slurry was then stirred for 15 minutes. After that, the slurry was dried in an oven at $100^{\circ} \mathrm{C}$ for 12 hours.

\subsubsection{Silica powder treated with NR serum protein}

About $0.45 \mathrm{~g}$ of NR serum protein was dispersed in $50 \mathrm{ml}$ of ethanol. The solution was stirred for 30 minutes. $5 \mathrm{~g}$ of silica was added to the solution slowly until all the silica was dispersed in the ethanol. The slurry was then stirred for 15 minutes. After that, the slurry was dried in an oven at $100^{\circ} \mathrm{C}$ for 12 hours.

\subsubsection{Silica powder treated with both silane and NR serum protein}

This procedure was the same as described above, only both $0.45 \mathrm{~g}$ of TESPT and $0.45 \mathrm{~g}$ of $\mathrm{NR}$ serum protein were dissolved in $50 \mathrm{ml}$ of ethanol.

\subsubsection{Glass slide treated with silane}

Before treatment the glass slides were cleaned by immersion in a $\mathrm{NaOH}(2.5 \mathrm{M})$ solution for 24 hours, followed by sonication in demineralized water for 10 minutes, then immersion in in $\mathrm{HCl}(0.5 \mathrm{M})$ for 15 minutes and sonication again in demineralized water for 10 minutes. The glass slides were then immersed in methanol for 5 minutes prior to silanization. The cleaned 
glass slide was dip coated in a silane solution (1\%wt TESPT in ethanol) for 15 minutes. After that, the glass slide was shaken in methanol for 5 minutes and rinsed in water for 10 minutes. The TESPT-coated glass slide was subsequently heated in an oven at $110^{\circ} \mathrm{C}$ for 15 minutes and stored in a vacuum desiccator.

\subsubsection{Glass slide treated with NR serum protein}

The cleaned glass slide was dip coated in protein solution $(1 \%$ wt NR serum protein in water). The glass slide was then rinsed in water for 10 minutes. After that, the proteincoated glass slide was heated in the oven at $110^{\circ} \mathrm{C}$ for 15 minutes and stored in a vacuum desiccator.

\subsubsection{Glass slide treated with both TESPT and NR serum protein}

The procedure was the same as described above, only the glass slides were dip-coated with a mixture of 50:50 wt\% of silane and protein solution from above.

\subsubsection{Analyses}

\subsubsection{Payne effect}

The Payne effect was investigated according to the method as described in the experimental section in Chapter 3.

\subsubsection{Bound Rubber Content measurements}

The Bound Rubber Content (BRC) was measured according to the method as described in the experimental section in Chapter 5 .

\subsubsection{Thermogravimetric Analysis (TGA) on bound rubber extracts}

TGA measurements were performed on the extracted dried materials of the bound rubber tests. A small amount of the dried insoluble rubber material was heated to $900^{\circ} \mathrm{C}$ in a thermal gravimetric analyzer (Perkin Elmer TGA 4000) with a heating rate of $30^{\circ} \mathrm{C} / \mathrm{min}$ under nitrogen flow.

\subsubsection{Contact angle measurements}

Static contact angle measurements were performed on treated glass slides while advancing contact angles were measured on treated silica powders using a Dataphysics OCA 40 Contact Angle Measuring System. The samples were measured according to the Sessile drop method by placing a water droplet $(5 \mu \mathrm{l})$ onto the sample with an electronically regulated syringe. For each sample, advancing contact angles were determined at three different spots using fresh deionized water for each new droplet. Experiments were performed at ambient temperature. 


\subsubsection{Kinetics of the silanization experiments}

For the kinetic study of silanization, masterbatches containing only rubber, silica, TESPT and oil were mixed in a Brabender Plasticoder 350S lab station using the same condition as above. The silanizations were carried out in a small Brabender 50 Labstation, at three different temperatures; 120,130 and $140^{\circ} \mathrm{C}$. The masterbatch was brought to the silanization temperature as fast as possible by increasing the rotor speed. After reaching the silanization temperature, the rotor speed was adjusted to keep the temperature level constant during the period of silanization. The silanization or reaction time was varied from $1,3,5$ to 20 minutes at the designated silanization temperature. The silanization at 20 minutes was selected as the time when the compound was considered to be fully silanized.

The samples were tested for the Payne effect immediately after the mixing. The reaction rates and activation energies for the silanization reaction were calculated according to ISO 53529 using the Payne effect data. The reaction constant for the first order reaction was calculated as follows:

$$
k_{r}=\frac{\ln \left(1-x_{1}\right)-\ln \left(1-x_{2}\right)}{t_{2}-t_{1}}
$$

The reaction variable, $x$ was calculated from the Payne effect at time $t, P E(t)$, the Payne effect of the starting material, $P E_{0}$ and the Payne effect for a fully silanized compound $P E_{\infty}$.

$$
x=\frac{P E(t)-P E_{0}}{P E_{\infty}-P E_{0}}
$$

The reaction constant, $k_{r}$ is given by the slope of the line of the logarithm of $(1-x)$ as a function of time. Based on equation 4 , the activation energy was determined from the slope of the line of the logarithm of $k_{r}$ as a function of the reciprocal of the product of absolute temperature $\mathrm{T}$ (in Kelvin) and gas constant $\mathrm{R}\left(8.314 \mathrm{JK}^{-1} \mathrm{~mol}^{-1}\right)$.

$$
k_{r}=k_{0} \exp \frac{-E_{a}}{R T}
$$

\subsection{RESULTS AND Discussion}

\subsubsection{Kinetics of silanization}

The kinetics of silanization for NR and IR are compared in Figure 7.1. The reaction rate constant, $\mathrm{k}_{\mathrm{r}}$, which is determined from the slope of the $\ln (1-\mathrm{x})$ versus time. For both rubbers, the silanization rate increases with raising the processing temperature, as seen from the decrease of the slope of $\ln (1-x)$ and higher $k_{r}$ with rise in temperature. At the temperature of $120^{\circ} \mathrm{C}$ the reaction rate for IR is slightly higher than for NR. The silanization rate for IR at 138 
130 and $140^{\circ} \mathrm{C}$ is somewhat lower than for NR. The silanization rate at $140^{\circ} \mathrm{C}$ for $\mathrm{NR}$ is $0.202 \mathrm{~min}^{-1}$ as compared to $0.1412 \mathrm{~min}^{-1}$ for IR.

(a) NR

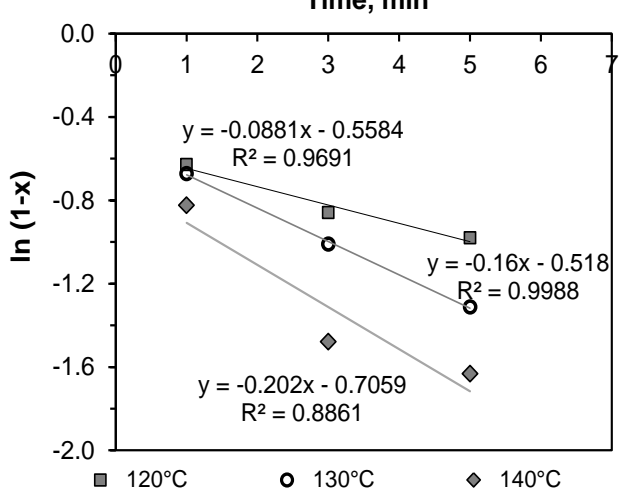

(b) IR

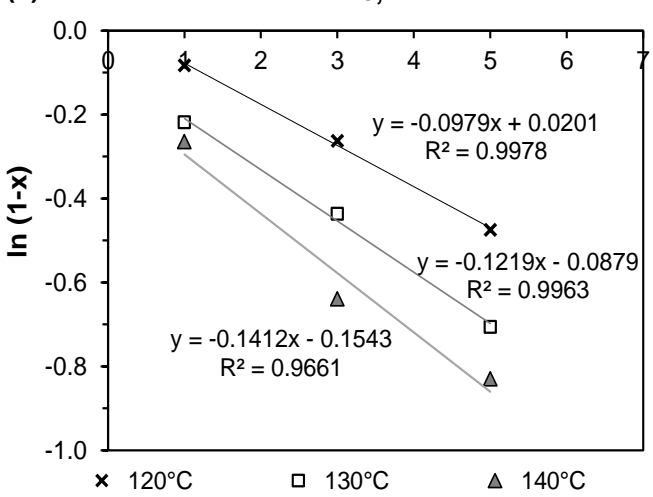

Figure 7.1 The logarithm of $(1-x)$ as a function of silanization time for (a) $N R$ and (b) IR at different temperatures, for determination of reaction constant.

(a) NR

1/RT x 1000

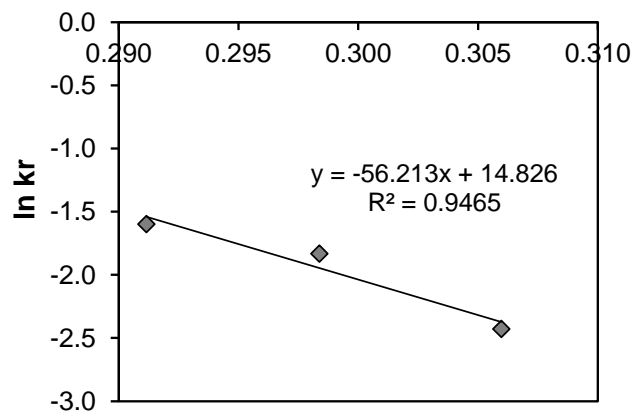

(b) IR

1/RT X1000

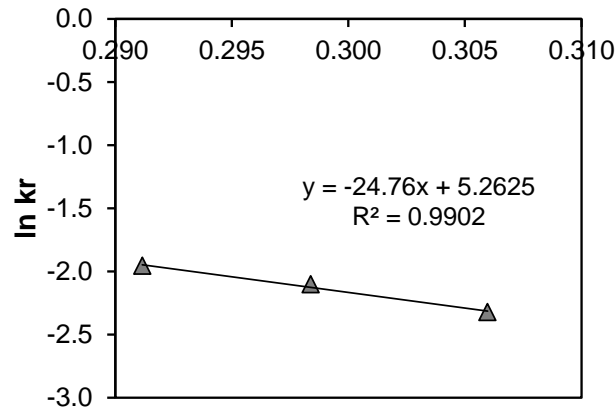

Figure 7.2 Determination of activation energy of silanization for NR and IR

The plot of silanization rate constants on logarithmic scale versus the inverse of temperature for NR and IR is shown in Figure 7.2. The activation energy of silanization for IR is $24 \mathrm{kJmol}^{-1}$, which is about half of the activation energy for NR: $56 \mathrm{kJmol}^{-1}$. As the amounts of silica and silane are the same for both IR and NR, the results show that the silanization of silica progresses differently. The lower activation energy for IR than for NR shows that the silica and silane reaction proceeds easier with IR with no interference of nonrubber. 


\subsubsection{Competitive interactions between silica-protein and silica-silane}

The addition of NR serum protein to NR- and IR-silica compounds helps to suppress the flocculation of silica as shown in Figure 7.3. For the NR-silica and IR-silica compounds the sign of flocculation is clearly observed from the cure curves as the extra "hump" at low cure time, but the compounds with NR serum proteins show much reduced agglomeration of silica. This is in accordance with the work in Chapter 4, where the presence of proteins in large quantities has the same effect as silane in hydrophobization of the silica surface. When NR serum protein and silane TESPT are added together in the IR-silica compound, only a small sign of flocculation is observed, but a marching modulus is obtained instead.

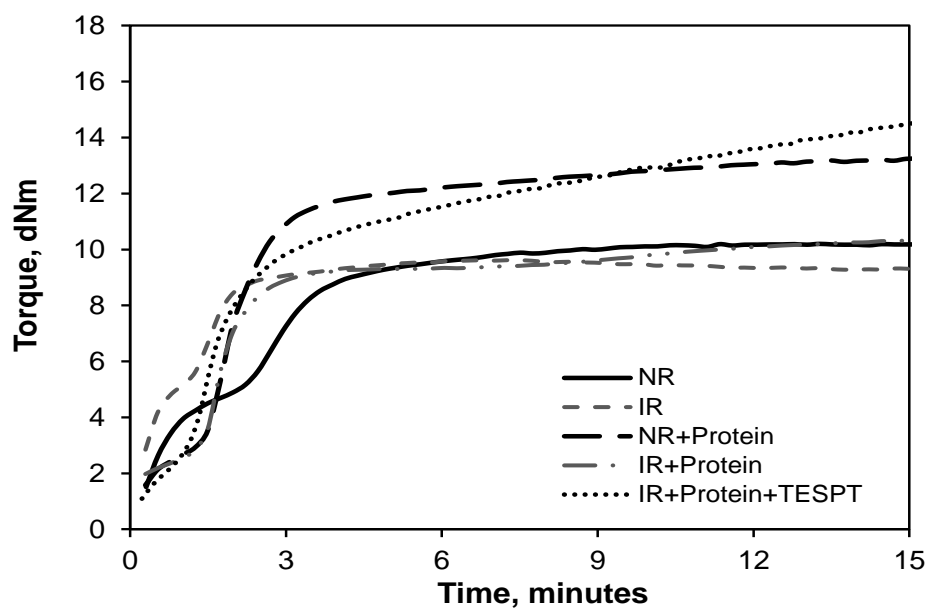

Figure 7.3 The effect of NR serum proteins on cure curves at $150^{\circ} \mathrm{C}$ for $N R$ - and IR-silica compounds.

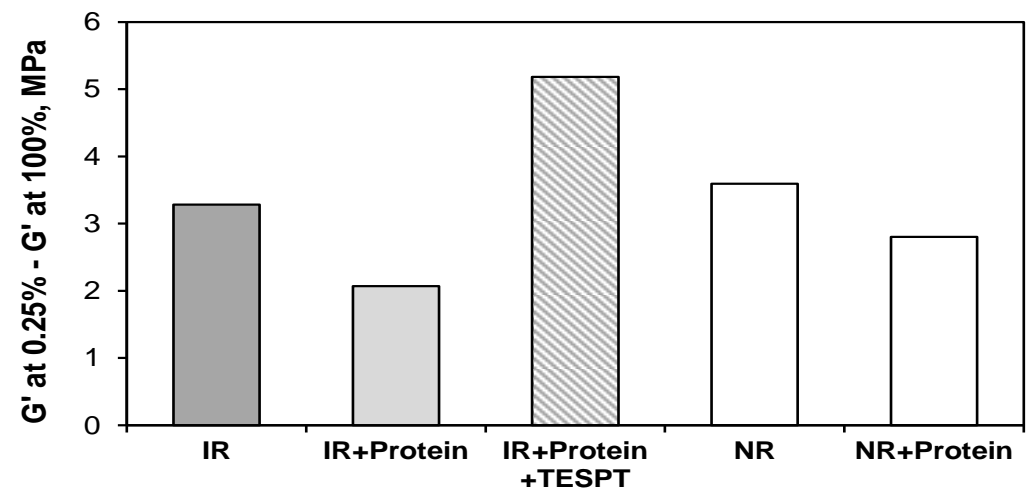

Figure 7.4 The effect of NR Serum proteins on Payne effect of IR and NR compounds. 
The effect of NR serum protein on hydrophobization of the silica surfaces is further illustrated in the Payne effect of the compounds in Figure 7.4. The filler-filler interactions in both NR and IR compounds are reduced when NR serum protein is added in the compound as demonstrated by the lower Payne effect. However, when NR serum protein and TESPT are added together in the IR-silica compound, the Payne effect is increased. The value of the Payne effect for the IR compound with NR serum protein and TESPT is even higher than for the compound without protein or silane. This indicates that when TESPT and proteins are both present in the system, they mutually reduce their efficiency to hydrophobize the silica surface. This shows the competition between NR serum protein and TESPT to interact with silica. The rise in the Payne effect of the silica-compound when the proteins and TESPT are both present has been observed already in Chapter 4 .

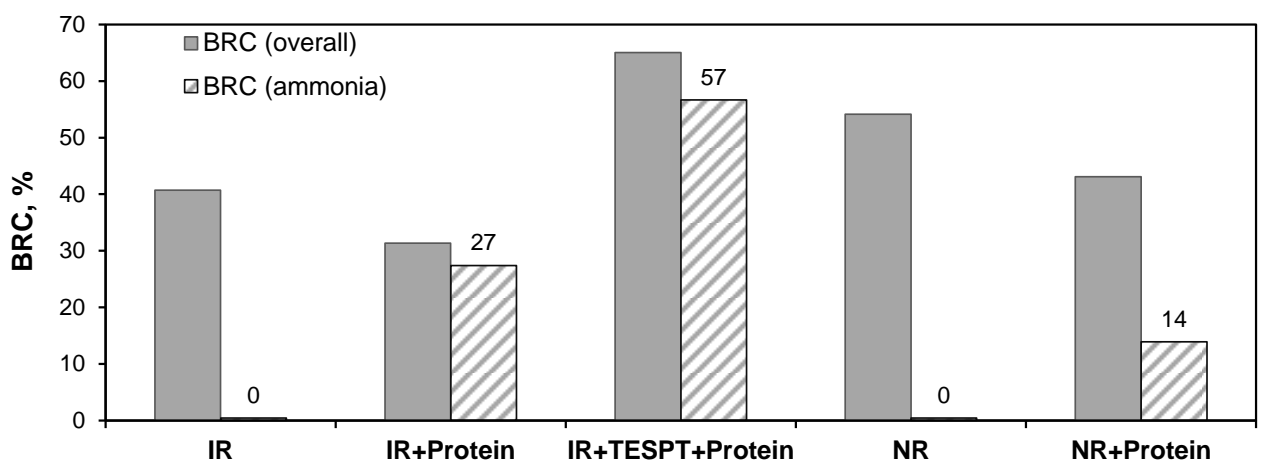

Figure 7.5 The effect of NR Serum proteins on bound rubber content of IR and NR compounds.

A comparison of bound rubber contents for NR and IR compounds is shown in Figure 7.5. The bound rubber content measured in normal atmosphere designates the total bound rubber content, while the bound rubber content in ammonia atmosphere refers to the chemically bound rubber. It seems that the total bound rubber content follows the same trend as the Payne effect in Figure 7.4. Even though NR serum proteins are able to disrupt silica-silica interactions, no improvement in the filler-to-rubber interaction in NR and IR compounds is observed from the slight reduction in total bound rubber content. What is still puzzling from Figures 7.4 and 7.5: the higher the filler-filler interaction, the higher is the total bound rubber content as well. It is noted that chemically bound rubber content is obtained for all compounds with NR serum proteins. For the IR compound with NR serum protein, 
about $90 \%$ of the total bound rubber is chemical of nature. The IR compound with both NR serum protein and TESPT also contains a similar high proportion of chemically bound rubber. In contrast, the NR compound with NR serum protein only exhibits about $30 \%$ of chemically bound rubber. The ammonia treatment of bound rubber breaks the hydrogen bonding in the compounds whether it is from silica-silica, silica-protein or protein-protein interactions. The reduction in the bound rubber content after ammonia treatment for the NR + serum protein compound shows that only part of the interactions are from hydrogen bonding. For IR compounds, most of the interactions are not derived from hydrogen bonding or otherwise should be destroyed during ammonia treatment.

The seemingly contradictory results thus far are different from the findings in Chapter 4, where the silica-filled skim rubber compound with no silane showed no chemically bound rubber content; and in Chapter 5 it was concluded that the interaction between silica and proteins in skim rubber mainly comes from hydrogen bonding. It needs to be pointed out therefore that the proteins that are present in skim rubber are different from NR serum proteins and this may give different interaction with the silica. The NR serum protein is obtained from the NR serum and already subjected to a processing step which involves drying, and this may lead to denaturation and changes in the conformational structure of the proteins. In addition, the proteins in skim rubber have more interactions with the rubber chain ends.

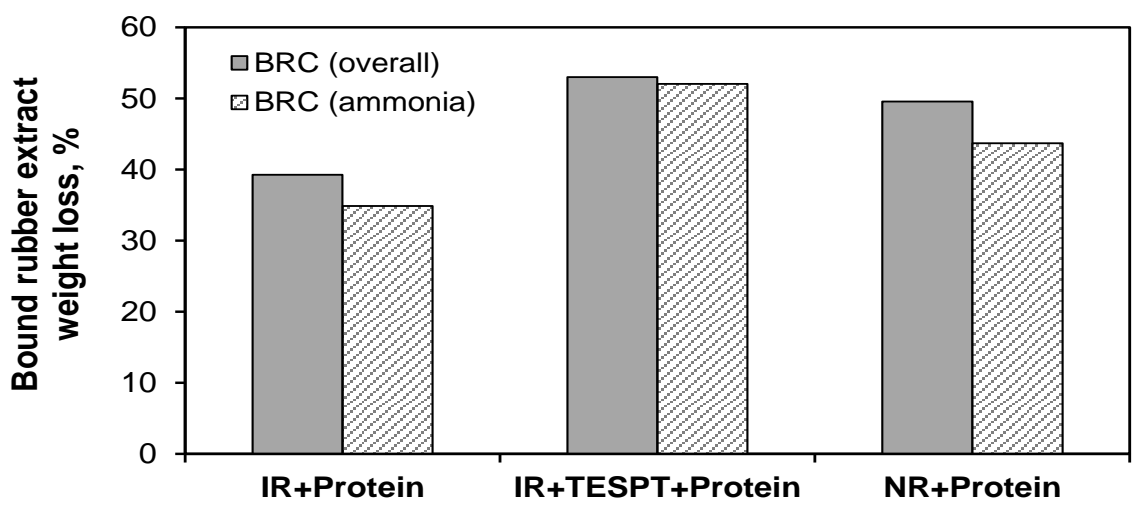

Figure 7.6 The TGA weight loss of the bound rubber extract of IR and NR compounds.

In order to confirm the existence of the bound rubber layer for the compounds with NR serum protein, TGA measurements were performed on the dried bound rubber extract: the filler with attached bound rubber. Figure 7.6 shows the TGA weight loss of the bound 142 
rubber extracts of the NR and IR compounds with NR serum protein. The weight loss represent the percentage of rubber in the bound rubber. The difference in weight loss between the bound rubber extract from normal and ammonia atmosphere is small. The bound rubber extract of NR with NR serum protein has a slightly higher amount of rubber as compared to the bound rubber extract of IR with NR serum protein. This indicates that NR has more interaction with silica as compared to the IR. The percentage of rubber in the bound rubber extract increases when NR serum protein is used in combination with silane TESPT. The amount of rubber in the bound rubber extract of IR+NR serum protein+TESPT compound is about $50 \%$.

Figure 7.7 shows the effect of NR serum protein on the stress-strain properties of the NR and IR compounds. The addition of NR serum protein to the silica-NR and silica-IR compounds clearly has a positive effect on the stress-strain properties of the vulcanizates. $A$ large increase in the moduli are observed when NR serum protein and TESPT are used in combination in the IR vulcanizate.

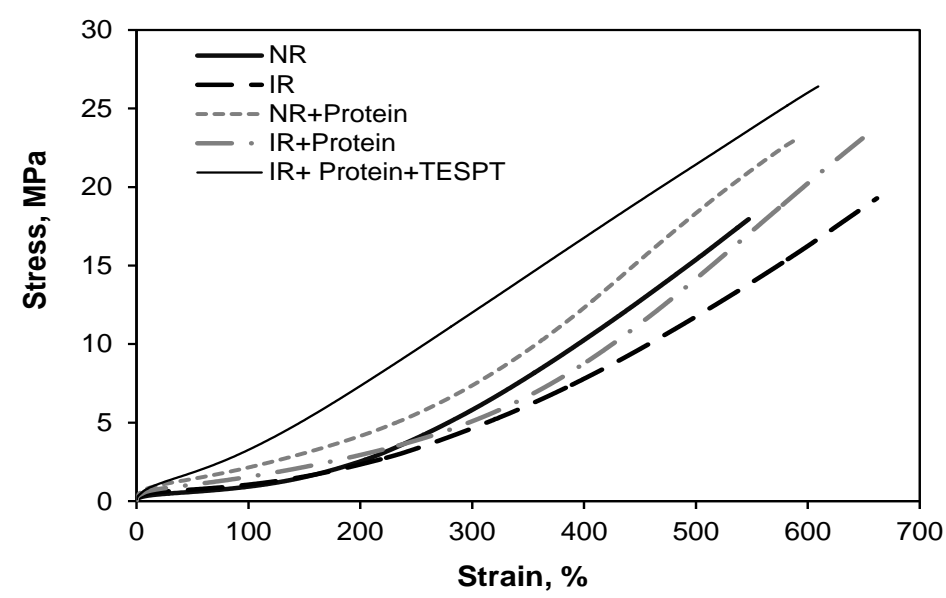

Figure 7.7 The stress-strain curves of IR and NR compounds as influenced by NR serum proteins.

\subsubsection{Adsorption of proteins on silica}

\subsubsection{Glass slides treated with TESPT vs. NR serum Protein}

Contact angle measurements were performed to study the effect of protein on the polarity of the silica surface. In this experiment a glass slide surface was used to imitate the silica surface. Furthermore, a flat glass slide surface is considered as an ideal surface, that is smooth, rigid and chemically homogeneous. Hence, the measured contact angle on a flat 
glass surface represents an ideal contact angle and is sometimes referred to as Young's contact angle ${ }^{16}$. A higher value of contact angle, $>90^{\circ}$, means the surface is hydrophobic, whereas a lower value of the contact angle, $<90^{\circ}$ represents a more hydrophilic surface.

Table 7.3 Contact angle of glass slide treated with silane and NR serum protein

\begin{tabular}{lc}
\hline & Advancing contact angle $\left(^{\circ}\right)$ \\
\hline Glass slide (untreated) & $30.5 \pm 0.5$ \\
Glass slide + TESPT & $39.0 \pm 5.0$ \\
Glass slide + NR serum Protein & $88.3 \pm 3.0$ \\
Glass slide + NR serum Protein + TESPT & $68.2 \pm 0.5$ \\
\hline
\end{tabular}

(a)

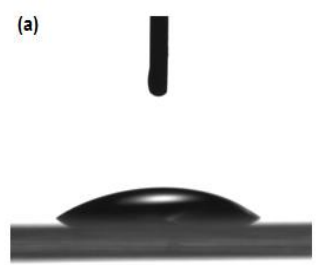

(b)

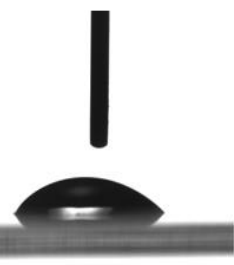

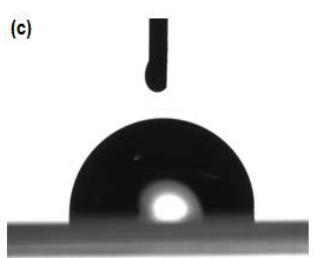

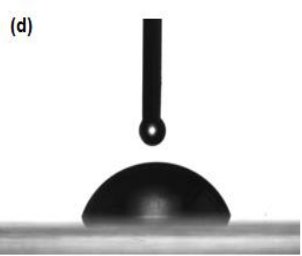

Figure 7.8 Representative figures of contact angle measurements of glass slide: (a) untreated; (b) treated with TESPT; (c) treated with NR serum protein; (d) treated with both TESPT and NR serum protein.

The contact angles, $\theta$ for the glass surfaces treated with NR serum protein, silane TESPT and both NR serum protein and TESPT are shown in Figure 7.8 and Table 7.3. The contact angle of the surface of the untreated glass slide is about $30^{\circ}$, and this value is close to that reported in literature, indicating a hydrophilic surface or partially hydrophilic ${ }^{17}$. Treating the glass slide with silane TESPT results in a slightly higher contact angle and this shows that, even though there is some hydrophobization by TESPT, the surface is still hydrophilic. When the glass slide is treated with TESPT, interaction and bonding between the ethoxy groups of TESPT and silanol groups on the glass slide are possible in parallel and perpendicular orientations as shown in Figure 7.9. As TESPT is a bis-functional silane, the hydrophobization will only increase in parallel orientation but if perpendicular orientation happens, there is only a small change in the hydrophilicity as ethoxy groups will cover the surface of the glass slide. Another explanation is that somehow the technique used to treat the surface is not sufficient to silanize the silica surface as it is done at low temperature. 
A large increase in hydrophobization of the glass slide is observed when it is treated with NR serum protein. The contact angle is close to $90^{\circ}$ implicating a strong hydrophobization effect of NR serum protein on the smooth silica surface. Possible interactions of proteins and the silica surface come from hydrogen bonding between carboxyl $(-\mathrm{COOH})$ and amino $\left(-\mathrm{NH}_{2}\right)$ groups of the proteins with silanol groups. As the silica surface is highly hydrophilic, the adsorption of NR serum protein to the glass slide by hydrophobic interaction can be ruled out. However, electrostatic interactions are possible, and this suggests that NR serum protein is oppositely charged. Another factor that determines the adsorption behavior of proteins is their structural stability. Protein undergoes structural changes upon adsorption. Protein that has a weak internal stability, or soft protein, tends to adsorb on all surfaces, driven by a gain in entropy arising from conformational changes. In this case, NR serum protein can be considered as consisting of soft proteins and the entropy gain overcomes unfavorable effects like hydrophilic and electrostatic repulsion between protein and the silica surface. A simple mechanism for protein adsorption on silica surface is illustrated in Figure 7.10 , which involves two steps ${ }^{8,18}$. In step 1 , protein reversibly adsorbs onto the silica surface. In step 2, surface-induced conformational changes take place due to the entropy gain. This conformational change is considered as irreversible, because the desorption rate decreases with increasing contact time.

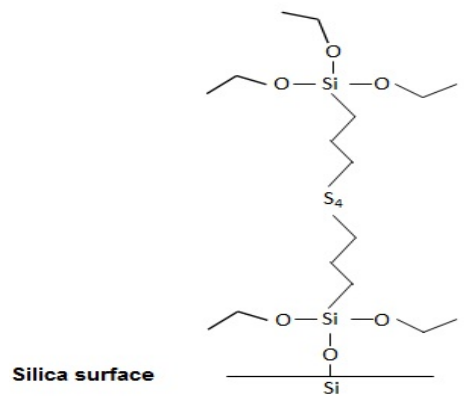

(a) perpendicular

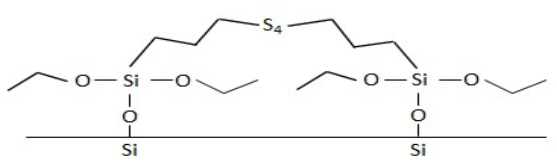

(b) parallel

Figure 7.9 Possible configurations of silane TESPT on the silica surface.

The contact angle for the glass surface treated with both TESPT and NR serum protein is up to about $70^{\circ}$, slightly lower than that treated with NR serum protein only. This points out that a slightly reduced hydrophobization effect of NR serum protein on silica surface occurs when TESPT is present. It also indicates the competitive effect of TESPT and protein to adsorb on the silica surface 

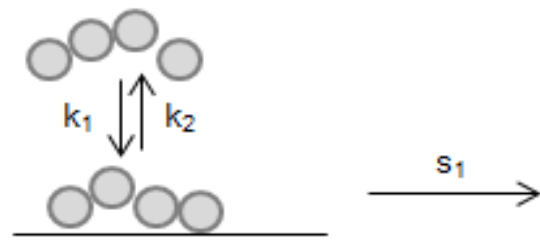

Figure 7.10 Mechanism for protein adsorption on silica surface.

\subsubsection{Silica powder treated with TESPT vs. NR serum protein}

A comparison of the hydrophobization of the surface of silica powder by proteins and silane coupling agent is shown in Figure 7.11. As the contact angle was measured on the silica powder, which is a rough surface, it is not an intrinsic equilibrium contact angle. The measured contact angle normally needs to be corrected to one of the known wetting regimes - Wenzel or Cassie to obtain the 'true' contact angle. However, no correction is done and only the measured contact is reported in this chapter as shown in Figure 7.12.

(a)

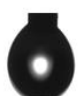

(b)
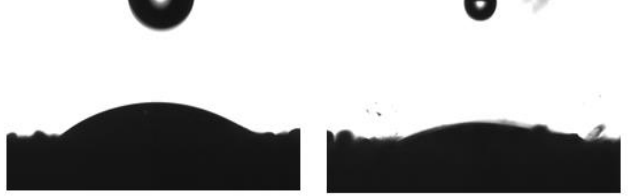

(d)

(c)
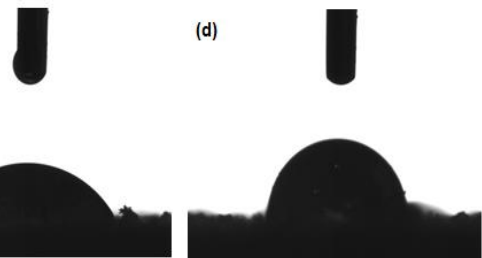

Figure 7.11 Contact angle measurements of silica powder: (a) untreated; (b) treated with TESPT; (c) treated with NR serum protein; (d) treated with both TESPT and NR serum protein.

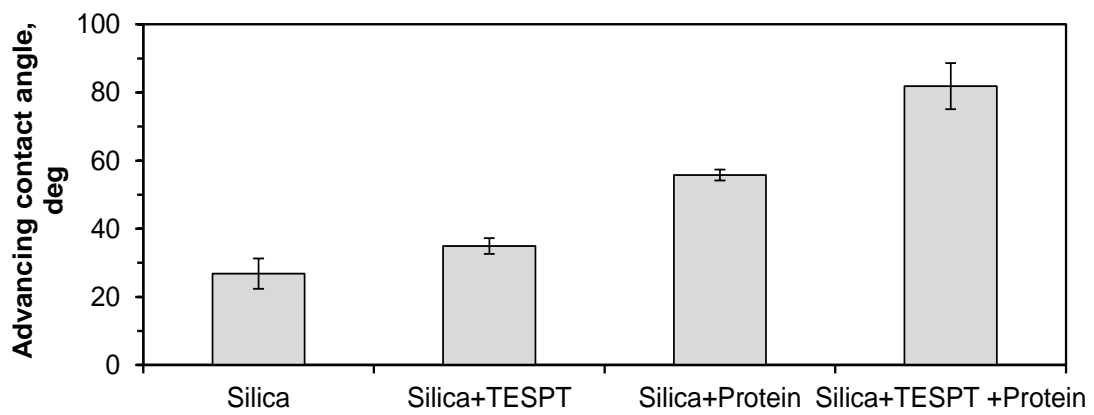

Figure 7.12 Contact angle for precipitated silica powder treated with TESPT and NR serum protein. 
Untreated silica powder has an advancing contact angle of approximately $35^{\circ}$, which is slightly higher compared to that obtained for the glass slide. Treatment of silica powder with TESPT results in more or less the similar contact angle. When the silica powder is treated with NR serum protein, a more hydrophobic surface is detected with a contact angle value of $55^{\circ}$, indicating the interaction of proteins with the silanol groups of silica surface. This is consistent with the results of contact angle for the glass slide. For silica treated powder, the contact angle is influenced by both the surface chemistry and also the surface roughness. In contrast, for a smooth surface such as for the glass slide, only the surface chemistry affects the contact angle.

The combination of NR serum protein and TESPT to modify the surface of the silica powder results in a higher contact angle which indicates higher hydrophobicity. This is different from the glass slide results, as the measurement is done on a rough surface. One explanation is that when part of the silica surface is covered by one side of the TESPT, the NR serum protein is also attracted to the unreacted ethoxy groups at the other end of the TESPT molecules. In addition, if perpendicular orientation of TESPT to the silica surface is favorable, it increases the area of adsorption for NR serum protein and hence higher hydrophobization is obtained. A comparison of the adsorption area occupied by protein on the silica surface when silane TESPT is present is shown in Figure 7.13.

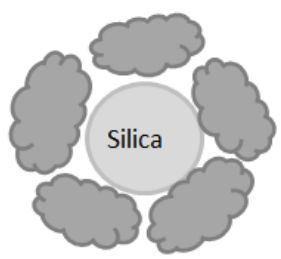

(a)

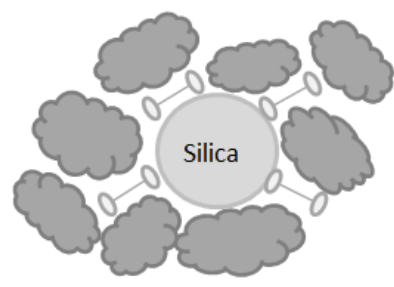

(b)

Figure 7.13 Schematic illustration of adsorption of protein on silica powder: (a) silica and $N R$ serum protein; (b) silica with both silane and NR serum protein.

Figure 7.14 shows a comparison of the advancing contact angle of the various treated silica powders as a function of time. For silica treated with TESPT, the water droplet penetrates into the surface at a very fast rate. It seems that the TESPT treated silica is more hydrophilic than untreated silica. Since the treatment is only done at $100^{\circ} \mathrm{C}$ for drying the coated silica powder, it is possible that only one side of TESPT is bonded to silica, and the tetarasulfide is not broken. Again, in this case it can be explained by the perpendicular 
orientation of TESPT on the surface of silica powder as in Figure 7.9 is favored. On the other side, the droplet-life-time for the silica treated with NR serum protein is longer. The same is observed for silica treated with the combination of NR serum protein and TESPT, indicating the hydrophobization of the silica surfaces. NR serum protein adsorbs on the surface of silica by changing its structure to adapt itself to the surface. In this case, as shown in Figure 7.13 (b), the NR serum protein is covering the surface of silica including the silane.

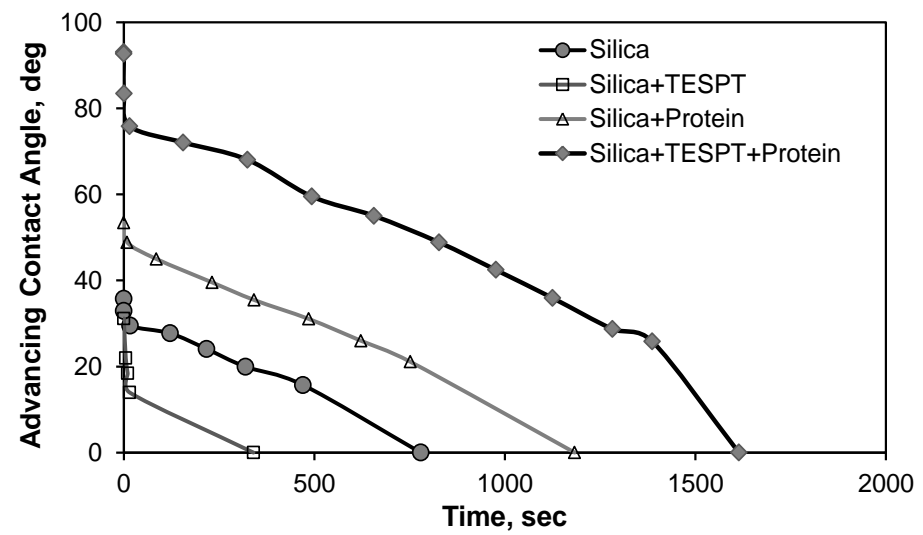

Figure 7.14 Advancing contact angle for silica powder treated with TESPT and NR serum protein.

\subsection{CONCLUSIONS}

The mechanism of proteins and silica interaction as compared to silane and silica interaction in hydrophobization of silica surfaces is illustrated. The kinetics of the silanization of silica is faster in synthetic polyisoprene (IR) than in natural rubber (NR), as demonstrated by the lower activation energy. An in-rubber study into the effect of NR serum proteins shows, that silica-protein interactions lead to reduced filler-filler interaction and suppress the flocculation of silica. However, a higher Payne effect is observed when NR serum protein is used in combination with silane TESPT, pointing to a competitive effect of protein and TESPT to hydrophobize the silica surface. The mechanism of proteins in hydrophobization of silica surfaces is demonstrated using a flat glass surface and compared to the silica powder. The contact angle measurement shows a strong hydrophobization effect of NR serum protein on the silica surface as compared to TESPT. For both glass surface and silica powder, TESPTtreated silica has a comparable contact angle to untreated silica. The contact angle of silica treated with NR serum protein is close to $90^{\circ}$, which shows the surface becomes hydrophobic. 


\subsection{References}

1. K.Nakanishi, Tahakaru Sakiyama and K. Imamura, J. Biosci. Bioeng., 91(3), 234 (2001).

2. J. J. Gray, Current Opinion in Biotechnology, 14, 110 (2004).

3. C. A. Haynes and W. Norde, Colloids Surface B. Biointerfaces, 2, 517 (1994).

4. V. Hlady and J. Buijs, Current Opinion in Biotechnology, 7, 72 (1996).

5. V.M. Gun'ko, I. V. Mikhailova, V.I. Zarko, I.I. Gerashchenko, N.V. Guzenko, W. Janusz, R. Leboda and S. Chibowski, J. Colloid Interface Sci., 260, 56 (2003).

6. S.S. Sarkawi, W.K. Dierkes and J.W.M. Noordermeer, Kautsch. Gummi Kunstst., 66(3), 27 (2013).

7. S.S. Sarkawi, W.K. Dierkes and J.W.M. Noordermeer, Rubber World, 247(2), 26 (2013).

8. V. Krisdhasima, J. Mcguire and R. Sproull, J. Colloid Interface Sci., 154, 337 (1992)

9. J. Buijs and V. Hlady, J. Colloid Interface Sci., 190, 171 (1997)

10. Y.I Tarasevich and L.I. Monakhova, Colloid Journal, 64(4), 482 (2002).

11. V.M. Gun'ko, V.V. Turov, V.I. Zarko, V.V. Dudnik, V.A. Tischenko, O.A. Kazakova, E.F. Voronin, S.S. Siltchenko, V.N. Barvinchenko and A.A. Chuiko, J. Colloid Interface Sci., 192, 166 (1997).

12. F.K. Onwu and S.P.I. Ogah, African Journal of Biotechnology, 10(15), 3014 (2011).

13. I.F. Mironyuk, V.M. Gun'ko, V.V. Turov, V.I. Zarko, R. Leboda, J. Skubiszewska-Zieba, Colloids Surface A, 180, 87 (2000)

14. K. Rezwan, L.P. Meier, I. J. Gaucklaer, Biomaterials, 26, 4351 (2005).

15. A.I. Ismail and Z. Mohd Nor, IIUM Engineering Journal: Special Issue on Biotechnology, 12(4), 61 (2011).

16. V. Dutschk and A. Marmur, in Colloid and Interface Chemistry for Nanotechnology, Ed., Chapter 12. In press

17. G. Vigil, Z. Xu, S. Steinberg and J. Israelachvili, J. Colloid Interface Sci., 165, 367 (1994).

18. P. Roach, D. Farrar and C.C. Perry, J. Am. Chem. Soc., 127, 8168 (2005). 


\section{Chapter 8}

\section{The Performance of Modified-Silica in Natural Rubber and Deproteinized Natural Rubber Compounds}

The reinforcement of pre-treated or compatibilizer/coupling agent surface-modified silica in Natural Rubber (NR) and Deproteinized NR (DPNR) is investigated. The use of modifiedsilica eliminates the need for coupling agent during mixing. The modified-silica is easily incorporated in NR during mixing as the silica surface has a higher hydrophobicity which enhances its compatibility with NR. The macro-dispersion of silica in NR is improved and silica flocculation is prevented with the use of modified-silica. The results show that there is no significant difference between the properties of NR and DPNR compounds filled with modified-silica The tensile strength, elongation at break and reinforcement index M300/M100 decrease with increasing silica loading. An increase in tan delta at $60^{\circ} \mathrm{C}$ and hysteresis energy loss for both NR and DPNR vulcanizates is proportional to the amount of modified-silica added. By using ATR-FTIR spectroscopy, the amount of silica in the compound can be monitored by the location of peak absorbance in the silica region between $1250-1000 \mathrm{~cm}^{-1}$. The peak of Si-O-Si stretch in both NR and DPNR vulcanizates is shown to shift to a lower wavenumber with increasing silica loading. 


\subsection{INTRODUCTION}

Compared to carbon black filled rubber, mixing of silica compounds involves many difficulties due to the large polarity difference between silica and rubber ${ }^{1}$. A bifunctional organosilane is commonly used as coupling agent in enhancing the compatibility of silica and rubber ${ }^{2}$. However, the in-situ reaction of silica and silane during rubber mixing needs an optimum high temperature, around $150^{\circ} \mathrm{C}$, for several minutes to ensure a complete silanization process ${ }^{3}$. For natural rubber (NR), this high mixing temperature is not advisable as degradation of NR can occur which will affect the final properties of the compounds ${ }^{4}$. Our previous work in Chapter 4 has established that silica can interact with proteins in the NR and lead to the silanization being partially hindered ${ }^{5}$.

One approach to overcome this difficulty for mixing NR and silica is using pretreated or surface-modified silica where the surface is already silanized to increase its hydrophobicity and hence ease the dispersion in the rubber matrix. The use of surfacemodified silica eliminates the need for a coupling agent and reduces volatile organic compound (VOC) emissions during mixing ${ }^{6,7}$. The commercial surface-modified precipitated silica, Agilon ${ }^{\mathrm{TM}} 400$ Performance silica, from PPG is a mercapto-organometallic and compatibilizer treated silica ${ }^{8-12}$ as shown in Figure $8 \cdot 1^{7,13}$. The surface of the modified silica has a mixture of coupling and non-coupling agents attached. The structure of silica Agilon $^{\mathrm{TM}} 400$ is similar to carbon black and conventional high-dispersible silica at aggregate size ${ }^{7}$.

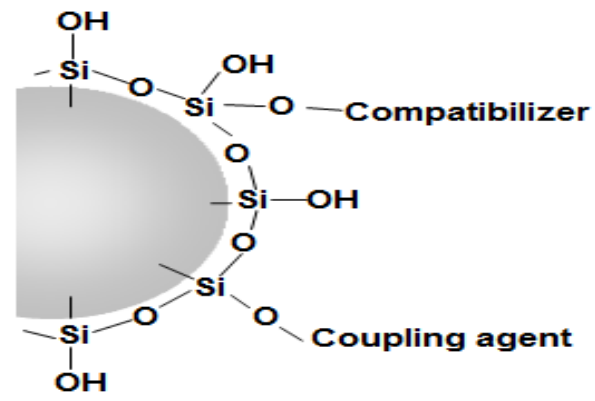

Figure 8.1 The different functionalities on the surface of pre-treated silica, Agilon ${ }^{\mathrm{TM}} 400$ Performance Silica ${ }^{7,13}$.

Mixing of silica Agilon ${ }^{\mathrm{TM}} 400$ and natural rubber can be carried out at lower dump temperature as the reaction between the silica and coupling agent has already been 
established. This gives the advantage to control the natural rubber degradation. In addition, the use of silica Agilon ${ }^{\mathrm{TM}} 400$ is expected to minimize the protein adsorption on the silica surface as the mercapto-functionality and compatibilizer already cover the silica surface and reduce the number of silanol groups.

In the present work, the reinforcement of a surface-modified silica, Agilon ${ }^{\text {TM }} 400$ in NR as well as DPNR compounds is investigated. The modified-silica loading in the compound is varied from 20 to 80 phr. Comparison of vulcanization properties, Payne effect, rubber-to-filler interaction, Mullins effect and physical properties between NR and DPNR compounds is discussed. In addition, the macro-dispersion of the silica and ATRFTIR spectroscopy of the compounds is also analyzed.

\subsection{EXPERIMENTAL}

\subsubsection{Materials}

Natural rubber (SMR20) and deproteinized natural rubber (DPNR) were used in this study. All experiments were performed using a tire tread composition based on the fuel-saving green tire technology, but with natural rubber as shown in Table 8.1. The surface-modified performance silica, Agilon ${ }^{\mathrm{TM}} 400$ from PPG Industries with CTAB surface area of $130 \mathrm{~m}^{2} / \mathrm{g}$ was used. The analytical data of performance silica Agilon ${ }^{\mathrm{TM}} 400$ are listed in Table 8.2. The silica content in the compound was varied from 20 to $80 \mathrm{phr}$.

Table 8.1 Compound formulation

\begin{tabular}{ll}
\hline Ingredients & phr \\
\hline Natural Rubber (NR \& DPNR) & 100 \\
Silica Agilon ${ }^{\text {TM }} 400$ & Varied $(20,55,60,70,75,80)$ \\
Zinc oxide & 2.5 \\
Stearic acid & 1 \\
Processing oil, TDAE & 8 or 10 (for $75 \& 80$ phr only) \\
Antioxidant, TMQ & 2 \\
Sulfur & 1.8 \\
CBS & 2.2 \\
DPG & 1.0 \\
\hline
\end{tabular}


Table 8.2 Typical properties of silica Agilon ${ }^{\mathrm{TM}} 400$

\begin{tabular}{ll}
\hline Parameter & Value \\
\hline CTAB surface area $\left(\mathrm{m}^{2} / \mathrm{g}\right)$ & 130 \\
Moisture, wt.\% & 6.0 \\
$\mathrm{pH}$ & 6.8 \\
$\mathrm{SH}$, wt.\% & 0.5 \\
Carbon, wt.\% & 4.0 \\
Skeletal Density & $2.02 \mathrm{~g} / \mathrm{cm}^{3}$ \\
Bulk density & $384 \mathrm{~kg} / \mathrm{m}^{3}$ \\
Form & Micro-granule \\
\hline
\end{tabular}

\subsubsection{Mixing}

The compounds were mixed in 2 steps. The first step mixing was done using a laboratory internal mixer Farrel Banbury BR1600, including the mastication step. The mixing procedure employed in the first step is indicated in Table 8.3. The fill factor of the mixer was fixed to $70 \%$, the starting temperature of the mixing chamber was $80^{\circ} \mathrm{C}$ and the rotor speed was 80 rpm. After mixing for 7 minutes, the masterbatch was sheeted out on a SKK 9"x18" two-roll mill to improve the dispersion of silica. After 24 hours, the curatives were added to the compound on the two-roll mill.

Table 8.3 Mixing procedure

\begin{tabular}{lll}
\hline $\begin{array}{l}\text { Step } 1 \\
\text { Time }\end{array}$ & Action & $\begin{array}{l}\text { Step 2 } \\
\text { Action }\end{array}$ \\
\hline 0.00 & Add rubber, mastication & All curatives were \\
1.00 & Add $30^{\text {a }}$ phr silica & mixed on a two roll \\
3.00 & Add $30^{\text {a }}$ phr silica, oil & mill \\
4.00 & Add remaining silica & \\
5.00 & Add ZnO, St.A, TMQ & \\
7.00 & Dump &
\end{tabular}

${ }^{a}$ The amount of silica additions depends on the total amount of silica for the compound. For 20 phr silica compound, only one addition of silica is applied.

\subsubsection{Sample analyses}

Mooney viscosity was measured at $100^{\circ} \mathrm{C}$ with a Mooney viscometer 2000E (Alpha Technologies) using the large rotor $(\mathrm{ML}(1+4))$ for compounds and the small rotor for 
masterbatches $(\mathrm{MS}(2+4))$. The vulcanization curves were measured using a MDR 2000 from Alpha Technologies, at temperatures of $150^{\circ} \mathrm{C}$.

Payne effect, tensile properties, hardness and tan delta at $60^{\circ} \mathrm{C}$ of the compounds were investigated according to the methods as described in the experimental section in Chapter 3. Bound rubber content was measured according to the methods as described in the experimental section in Chapter 5.

Vulcanizates were prepared by curing the compounds for their respective t95 $_{95}$ (time to reach $95 \%$ of torque difference in the curemeter) at $150^{\circ} \mathrm{C}$ using a Wickert laboratory press WLP $1600 / 5^{\star} 4 / 3$ at 100 bar.

The Mullins effect and hysteresis loss of vulcanizates were determined on tensile dumbbells (ISO-37: type 2) at room temperature using a Zwick Z020 tensile tester. The measurements were done at $300 \%$ extension for 5 cycles with the same crosshead speed of $200 \mathrm{~mm} / \mathrm{min}$ for loading and unloading.

The macro-dispersion of the silica was measured using a Dispergrader Optigrade reflective optical light microscope from Alpha Technologies. The vulcanized samples were cut with a razor blade. The magnification of the microscope was 100 times. Agglomerate sizes of $3 \mu \mathrm{m}$ and larger can be detected with this method.

Infrared spectra of vulcanizate samples were recorded using a Fourier Transform Infrared (FTIR) Perkin Elmer 100 series system with a Attenuated Total Reflection (ATR) attachment. All spectra were collected at $4 \mathrm{~cm}^{-1}$ resolution, 10 scans per sample, scan range of $4000-650 \mathrm{~cm}^{-1}$, and subtracting the background at the beginning of the test. The spectra were analyzed without correction. The location of the peak absorbance near 1000 $\mathrm{cm}^{-1}$ was determined using the Spectrum 100 find peak tool, while the area of the absorbance spectra in the region $1250-850 \mathrm{~cm}^{-1}$ was calculated using the Spectrum 100 area tool.

\subsection{Results AND Discussion}

\subsubsection{Macro-dispersion of silica compounds}

The macro-dispersion of modified-silica in NR and DPNR vulcanizates at varying filler loadings is represented in Figure 8.2. The image is based on 100 times magnification. The macro-dispersion of modified-silica is clearly seen from the white spots in the vulcanizates with 20 phr of silica. It seems that the macro-dispersion of silica Agilon ${ }^{\mathrm{TM}} 400$ is improved at higher filler loadings as shown by the decrease in the white area percentage and reduction in agglomerate size in Figure 8.3. Generally, DPNR vulcanizate exhibits better macrodispersion of modified-silica as compared to NR vulcanizate. 


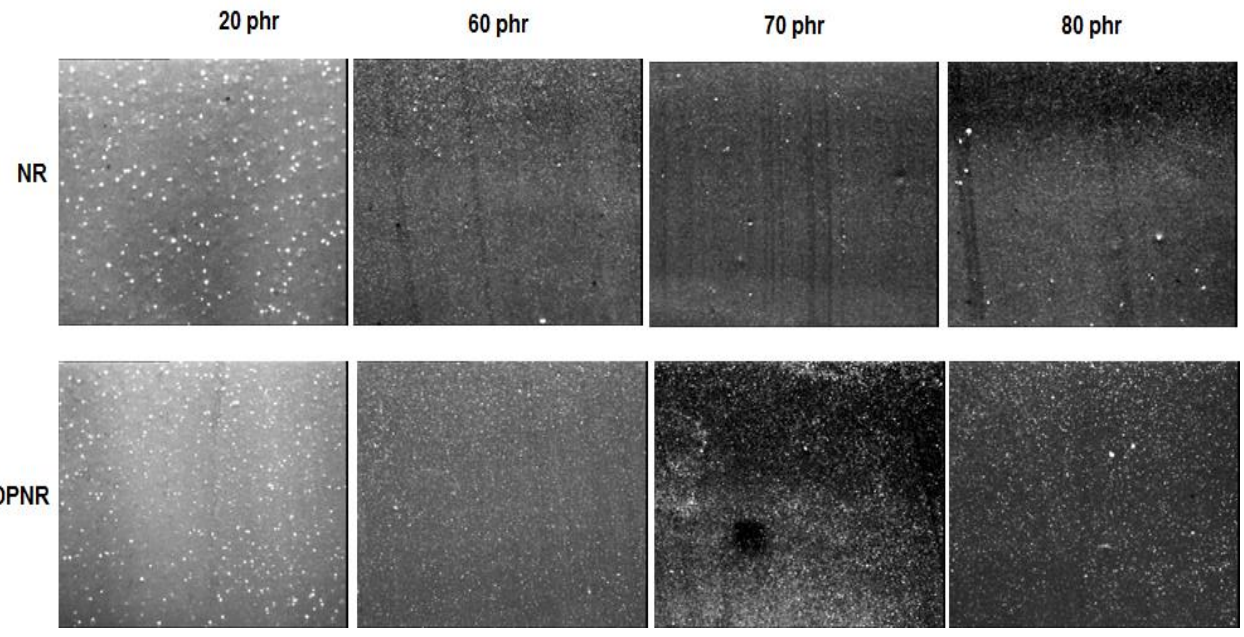

Figure 8.2 Comparison of macro-dispersion of modified-silica in NR and DPNR compounds.
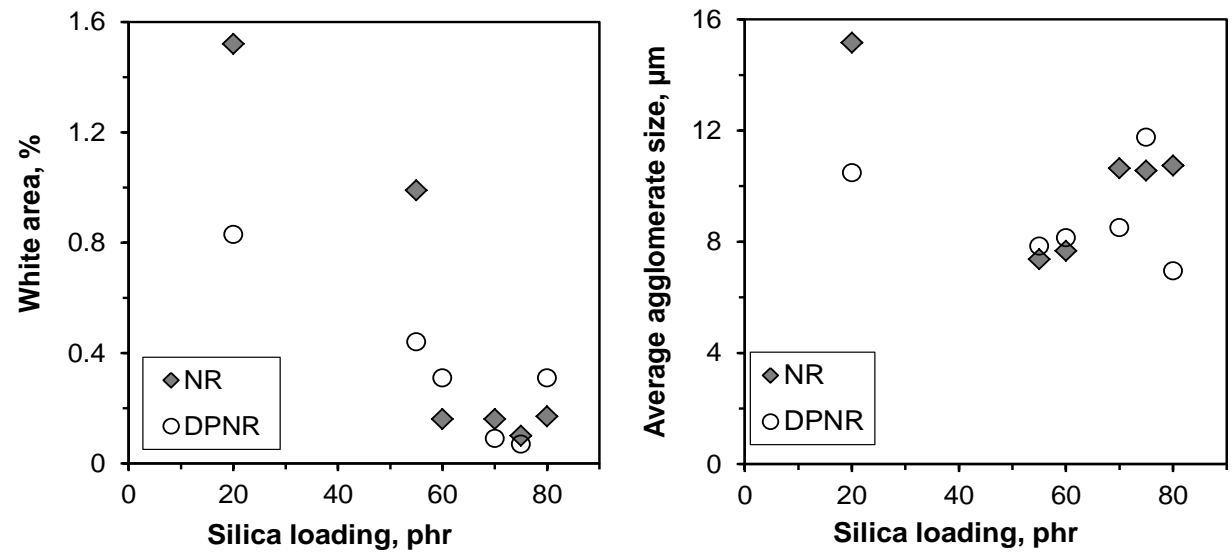

Figure 8.3 White area percentage and average agglomerate size as a function of modifiedsilica loading in NR and DPNR compounds.

\subsubsection{Effect of silica loading on vulcanization characteristics}

Comparison of the vulcanization characteristics at $150^{\circ} \mathrm{C}$ of NR and DPNR compounds containing modified-silica at varying loading is shown in Figure 8.4. Increasing the silica loading from 20 to $55 \mathrm{phr}$ significantly increases the maximum torque of both NR and DPNR compounds. This can be attributed to the higher amount of modified-silica dispersed in the rubber matrix and thus giving a higher extent of reinforcement. At filler loadings between 60 to $80 \mathrm{phr}$, the torque difference is almost comparable. At high filler loadings, the dispersion of the modified-silica apparently become difficult as the amount of filler reaches saturation 156 
and hence shows only a relatively small increase in maximum torque. The use of modifiedsilica (Agilon ${ }^{\mathrm{TM}} 400$ ) helps to avoid flocculation of the silica which is normally seen at the beginning of vulcanization. The vulcanization curves for both NR and DPNR compounds show no signs of flocculation even at very high loadings of modified-silica.
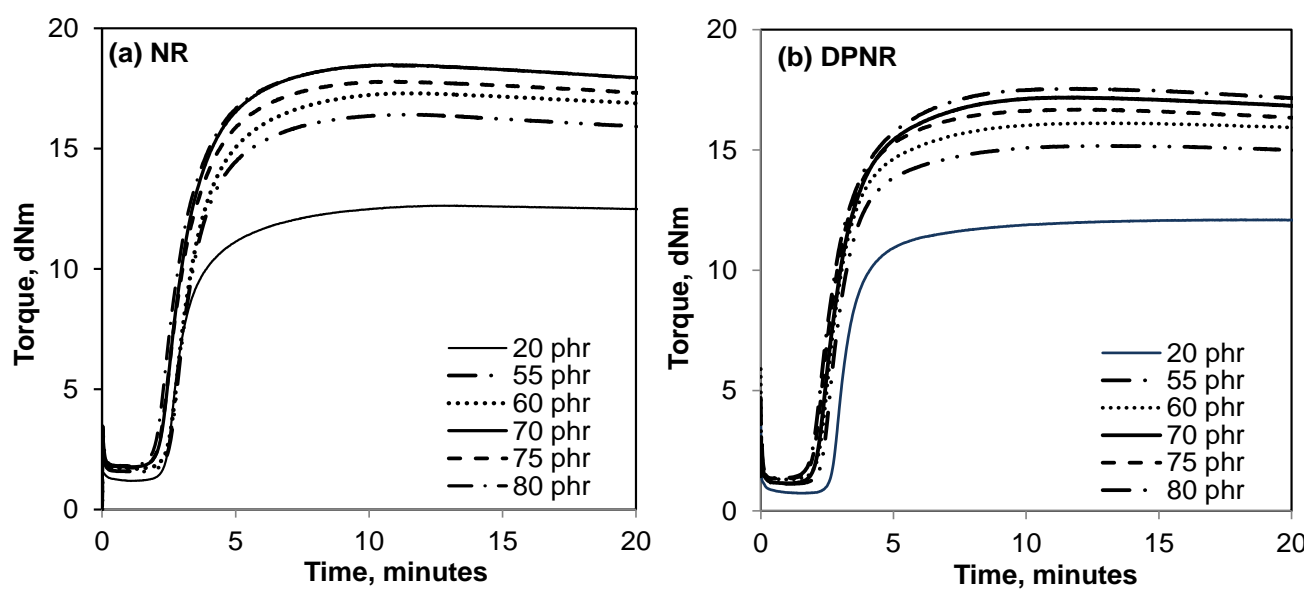

Figure 8.4 Cure characteristics at $150^{\circ} \mathrm{C}$ of (a) NR and (b) DPNR compounds at varying silica loadings.

\subsubsection{Processability of the silica compounds}

The Mooney viscosities of the modified silica compounds with and without curatives increase with increasing filler loadings as shown in Figure 8.5. In both masterbatches and compounds, the Mooney viscosities for DPNR are about $20 \%$ lower than those for NR. This may indicate easier processability for the DPNR compounds. In Figure 8.6, the Mooney scorch of the silica compounds is seen to decrease with increasing silica loading. However, the effect of the silica loading on reduction of scorch time is not observed from the vulcanization curves as discussed in the previous section, because the decrease is relatively small. 

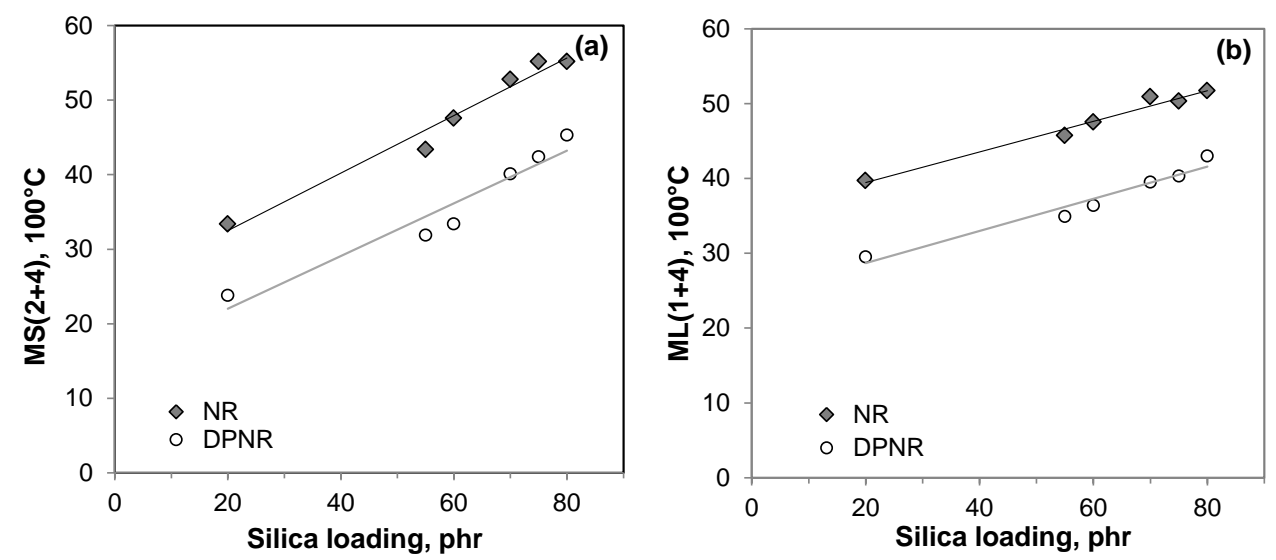

Figure 8.5 Comparison of Mooney viscosity of (a) masterbatches and (b) compounds of NR and DPNR filled with modified silica.

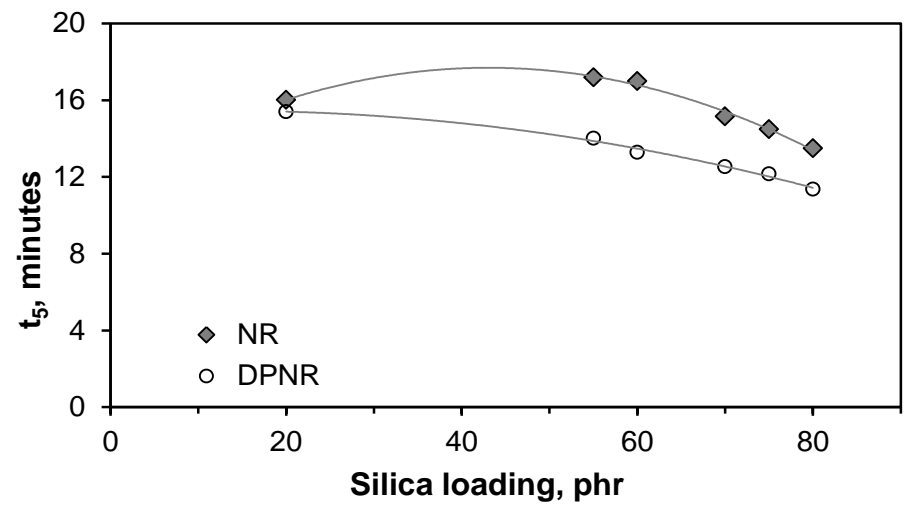

Figure 8.6 Effect of silica loading on Mooney scorch of NR and DPNR compounds.

\subsubsection{Payne effect and filler-filler interactions in the modified-silica compounds}

The surface of silica Agilon ${ }^{\mathrm{TM}} 400$ is modified with mercapto-organometallic and noncoupling agent as shown in Figure 8.1. Due to this surface activity, the silica networking is low for this modified-silica. As shown in Figures 8.7 and 8.8, the Payne effect of silica-filled NR and DPNR show similar behavior where it increases with increasing filler loading. Even though the surface of the silica is modified, there are still silanol groups present. At high amounts of silica, the distance between the aggregates becomes shorter, silica-silica interaction increases and the possibility of silica networking to occur is high. In comparison, DPNR compounds show slightly lower filler-filler interaction than NR. However, the difference diminishes after vulcanization. 


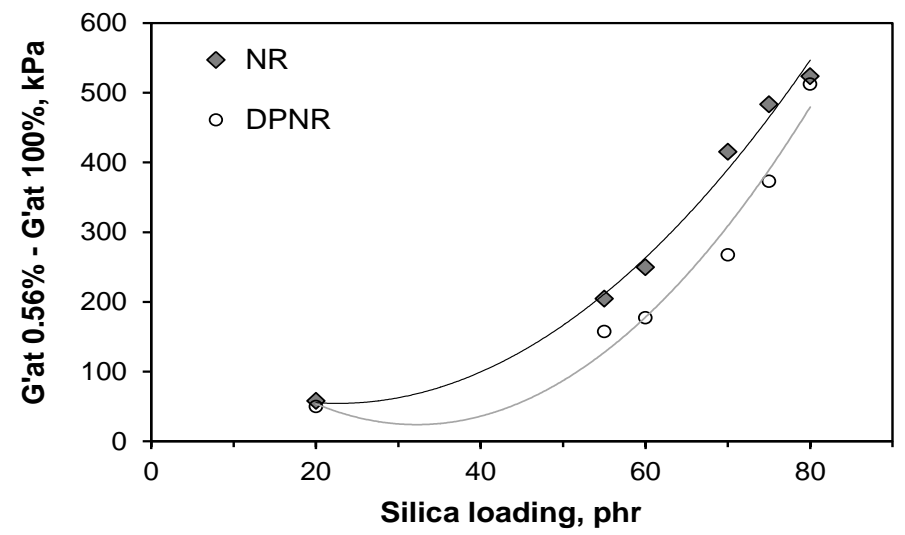

Figure 8.7 Payne effect of the masterbatches of silica filled NR and DPNR.
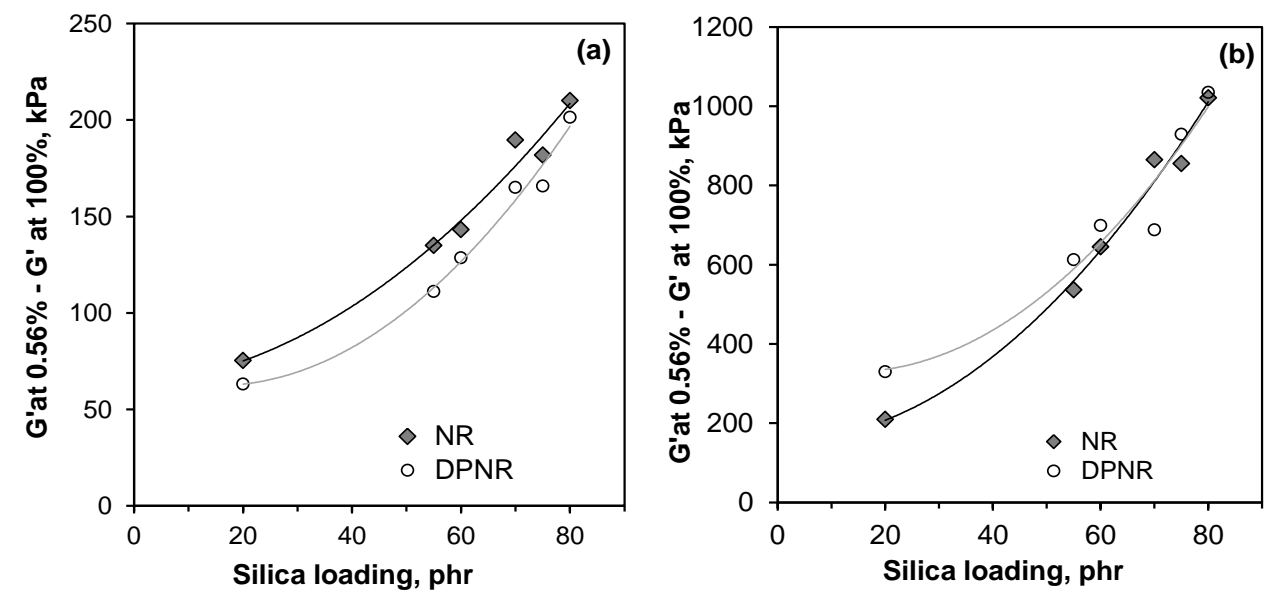

Figure 8.8 Comparison of Payne effect of silica in NR and DPNR compounds (a) before and (b) after vulcanization.

\subsubsection{Monitoring filler-filler interaction using ATR-FTIR}

The interactions of silica-silica filler can be characterized by ATR-FTIR spectroscopy. Silica has an absorbance region between $1250-1000 \mathrm{~cm}^{-1}$. The FTIR spectra of the silica region of NR and DPNR vulcanizates are shown in Figures 8.9 and 8.10 , respectively. The asymmetric Si-O-Si stretch peak is located between 1087 to $1066 \mathrm{~cm}^{-1}$. For low silica loadings, shoulders are observed around 1030 and $1017 \mathrm{~cm}^{-1}$. The peak of the silica absorbance in both NR and DPNR vulcanizates is shown to shift to a lower wavenumber with increasing modified-silica loading. This is in agreement with the work reported by Kralevich and Koenig ${ }^{14}$. In Figure 8.11(a), both NR and DPNR vulcanizates show the same 
relationship between the peak centers of the silica region and modified-silica loading. For an increase of modified-silica of $10 \mathrm{phr}$, the peak of the silica region wavenumber decreases by $3.5 \mathrm{~cm}^{-1}$. A close correlation between the peak center of the silica region and the Payne effect is observed, as shown in Figure 8.11(b). With increasing silica loading, the filler-filler interaction is enhanced and this results in the shift of the peak center of the silica region in FTIR.

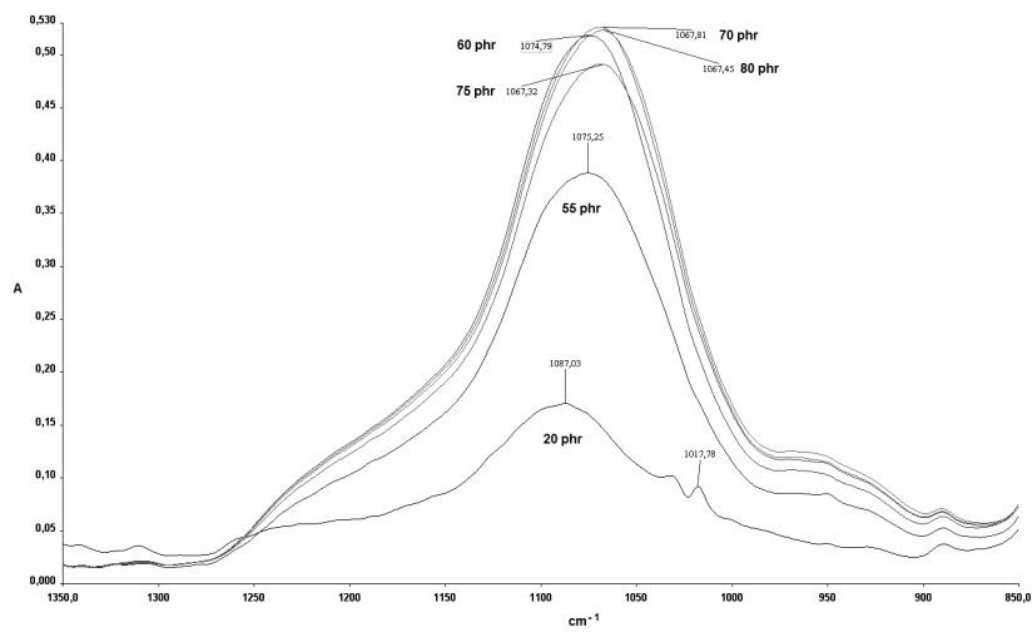

Figure 8.9 The ATR-FTIR spectrum of the asymmetric Si-O-Si stretch in the silica region in $N R$ vulcanizates.

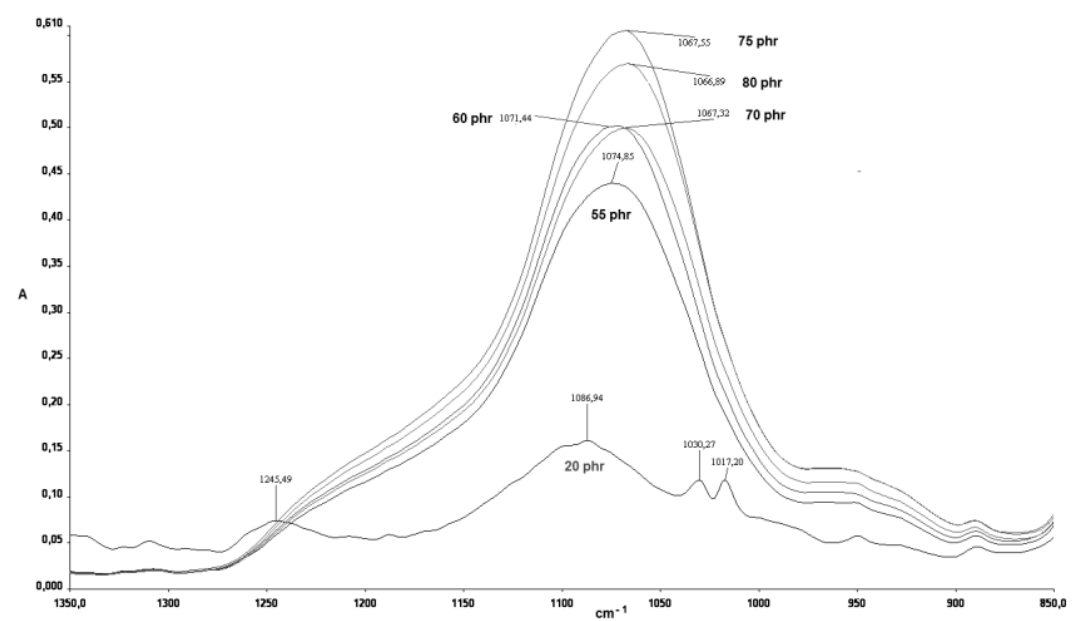

Figure 8.10 The ATR-FTIR spectrum of the asymmetric Si-O-Si stretch in the silica region of DPNR vulcanizates. 

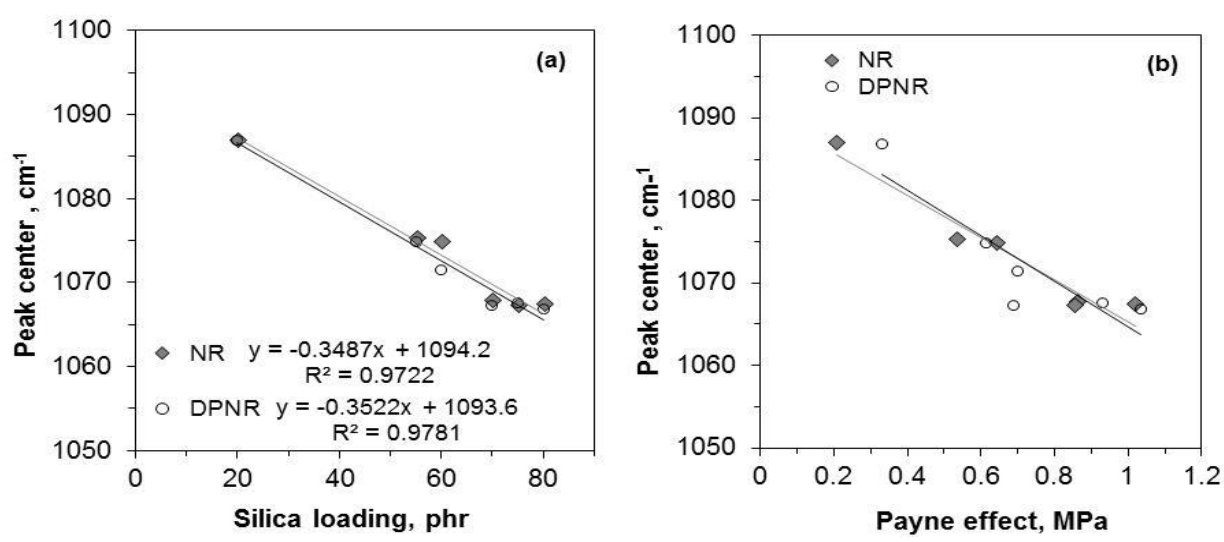

Figure 8.11 (a)The effect of silica loading on the location of the peak center of Si-O-Si stretch in NR and DPNR vulcanizates; (b)The correlation between the shift in the location of peak center of Si-O-Si stretch and the Payne effect of NR and DPNR vulcanizates.

The intensity of the peak also rises with increment in silica loading but only up to 70phr. The calculated peak area of the silica region of NR vulcanizates as compared to DPNR vulcanizates is presented in Table 8.4. At high loading of silica, there is inconsistency in the intensity of the peaks due to the restricted degree of silica dispersion in the rubber matrix. A good micro-dispersion of this silica at loadings above $70 \mathrm{phr}$ is apparently difficult to achieve as the silica-silica interactions become prevailing.

Table 8.4 Absorption area in the range of $1250-1000 \mathrm{~cm}^{-1}$ for NR and DPNR vulcanizates with varying modified-silica loading

\begin{tabular}{ccc}
\hline Silica loading (phr) & NR $\left(\right.$ area cm $\left.\mathrm{cm}^{-1}\right)$ & DPNR $($ area cm \\
\end{tabular}

\subsubsection{Rubber-to-filler interactions}

Bound rubber, a measure of filler surface activity and rubber-filler interactions, is an important factor in filler reinforcement of rubber. Rubber chains are attracted either physically or chemically to form bound rubber on the surface of silica. In Figure 8.12, the 
chemically and physically bound rubber contents (BRC) of NR and DPNR compounds at varying modified-silica loadings is compared. Since Agilon ${ }^{\mathrm{TM}} 400$ is silanized with a mixture of coupling and non-coupling agents, it is expected that at $20 \mathrm{phr}$ silica there is no chemically BRC obtained (before vulcanization) for both NR and DPNR compounds, as it will behave like untreated silica without coupling agent. It is surprising however to see that NR compounds show a consistently high chemically bound rubber at silica loadings between 55 to 70 phr, while DPNR-silica compounds only exhibit chemically BRC at and above 70phr silica loadings.
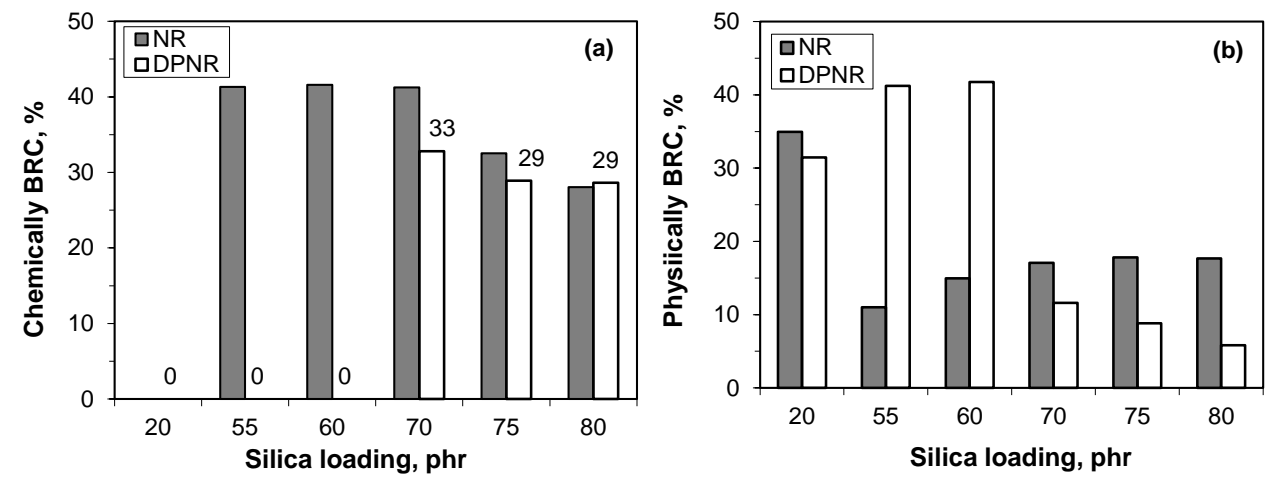

Figure 8.12 Chemically and physically bound rubber content of NR and DPNR filled with modified silica at varying loadings.

It seems that there are more rubber-to-filler interactions in the NR compounds as compared to the DPNR compounds regardless of the same polyisoprene structure and unsaturation. The major difference between the NR and DPNR is the purification of DPNR from non-rubber constituents, particularly proteins. The chemically bound rubber observed in the NR compounds may therefore be attributed to interactions of silica and proteins. However, our previous work on silica-filled NR compounds without coupling agent resulted in no chemically bound rubber for both NR and DPNR compounds ${ }^{15}$. Since the surface of silica Agilon ${ }^{\mathrm{TM}} 400$ is treated with non-coupling agent and compatibilizer, it also possible that the reactivity of NR is higher towards the mercapto-functionality and the compatibilizer. At and above $70 \mathrm{phr}$ silica, the chemically BRC of NR compounds is shown to drop slightly. It seems that there is an optimum amount of silica in NR giving the high rubber-to-filler interactions. When compared to NR compounds, the value of chemically BRC of the DPNR compounds is somewhat lower. 


\subsubsection{Hysteresis and Mullins effect of silica-filled NR and DPNR vulcanizates}

Hysteresis of rubber is the amount of energy dissipated during a cyclic deformation. When rubber is subjected to cyclic loadings, a stress required in the first stretch is greater than in the subsequent stretches. This stress softening phenomenon is called the Mullins effect. This Mullins effect occurs in filled and unfilled rubber, whether it is vulcanized or unvulcanized $^{16}$. However, the Mullins effect is most pronounced in filled vulcanizates. This is attributed to the breakdown of both filler aggregates and agglomerates and loss of adsorption of the filler particles to the rubber.

Both NR and DPNR vulcanizates show similar Mullins effects at the same silica loading as depicted in Figure 8.13 for 60 phr. The stress softening effect of NR and PDNR filled with modified-silica can be observed with pronounced lowering in the stress in the second and subsequent cycles. The hysteresis loss, which is calculated as the area of the hysteresis loop, decreases drastically in the second cycle and after the fourth cycle the value reaches a plateau.
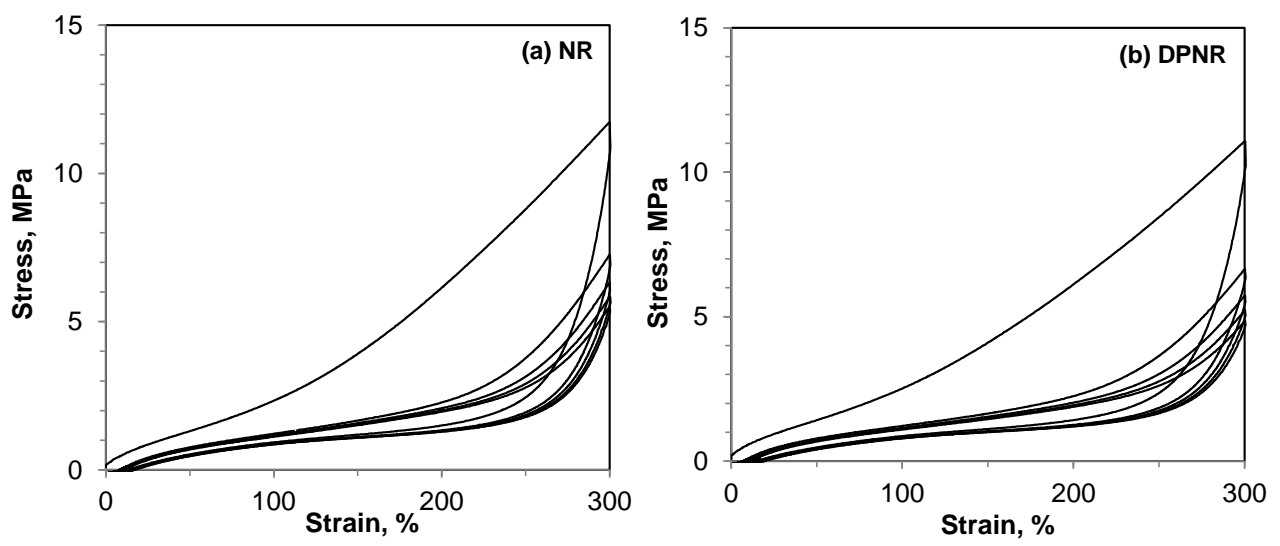

Figure 8.13 Comparison of Mullins effect in NR and DPNR filled with 60 phr of modified silica. 5 cycles at $300 \%$ strain.

Increasing the filler loading increases the hysteresis of rubber both hydrodynamically and viscoelastically. The influence of silica loading on hysteresis of NR and DPNR vulcanizates is pictured in Figure 8.14. A drastic increase in energy loss is observed when the amount of modified-silica used is tripled from 20 to 60 phr. This is in agreement with Payne effect results where the filler-filler interactions are strong at high loadings of silica. In Figure 8.15, the hysteresis of DPNR vulcanizates is proportional to the silica loading. However, for NR vulcanizates the energy loss shows a plateau at and above 
$70 \mathrm{phr}$ of silica Agilon 400. This may relate to the reduction in rubber-to-filler interaction at high loading of the modified-silica as also seen from the lower chemically bound rubber and higher Payne effect.
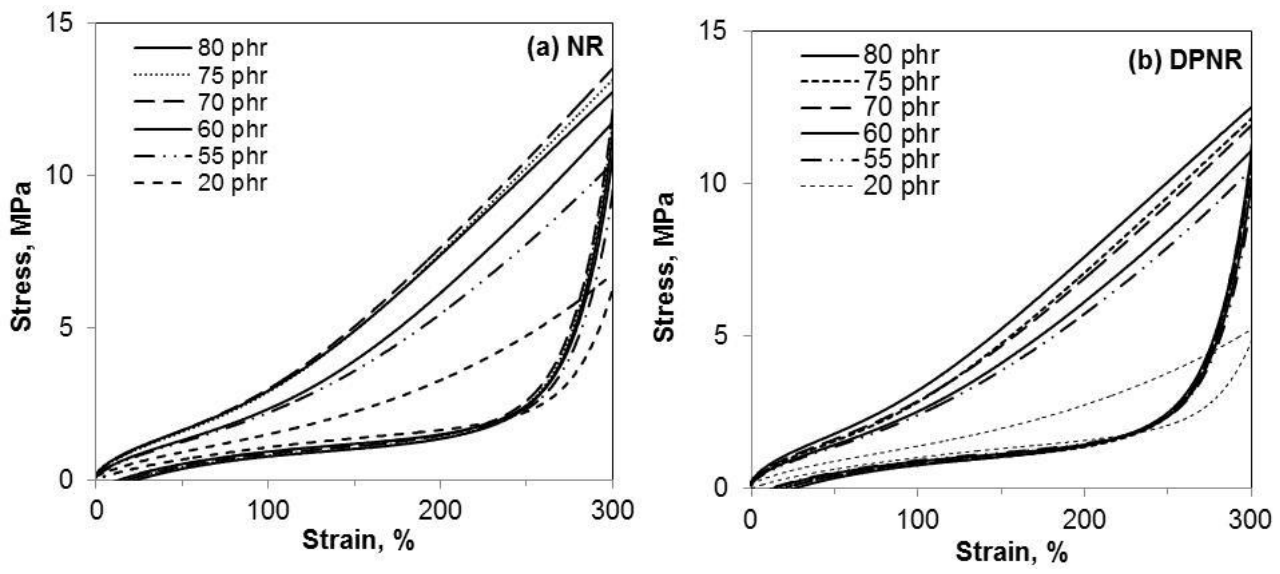

Figure 8.14 Hysteresis of NR-modified silica vulcanizates in the first cycle at varying filler loading.
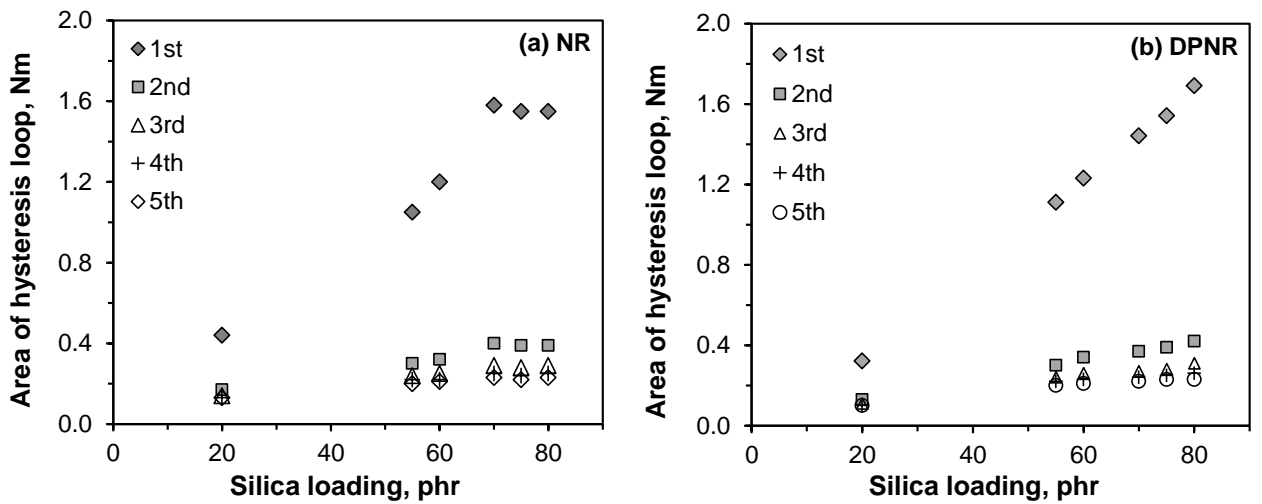

Figure 8.15 The effect of silica loading on the area of hysteresis loop: (a) NR and (b) DPNR vulcanizates.

\subsubsection{Mechanical properties of modified-silica vulcanizates}

The mechanical properties of the silica-filled NR and DPNR are influenced by the filler loading. Figure 8.16 represents a comparison of the mechanical properties of NR and DPNR compounds reinforced with different loadings of modified-silica. The results show that there is no significant difference in the mechanical properties between NR and DPNR 
vulcanizates filled with modified-silica. Both NR and DPNR vulcanizates exhibit the same trends where the tensile strength and elongation at break reduce slightly with increasing silica loading. In addition, the moduli are raised when higher amounts of modified-silica are present in the matrix. The hardness is also influenced by the filler loading.
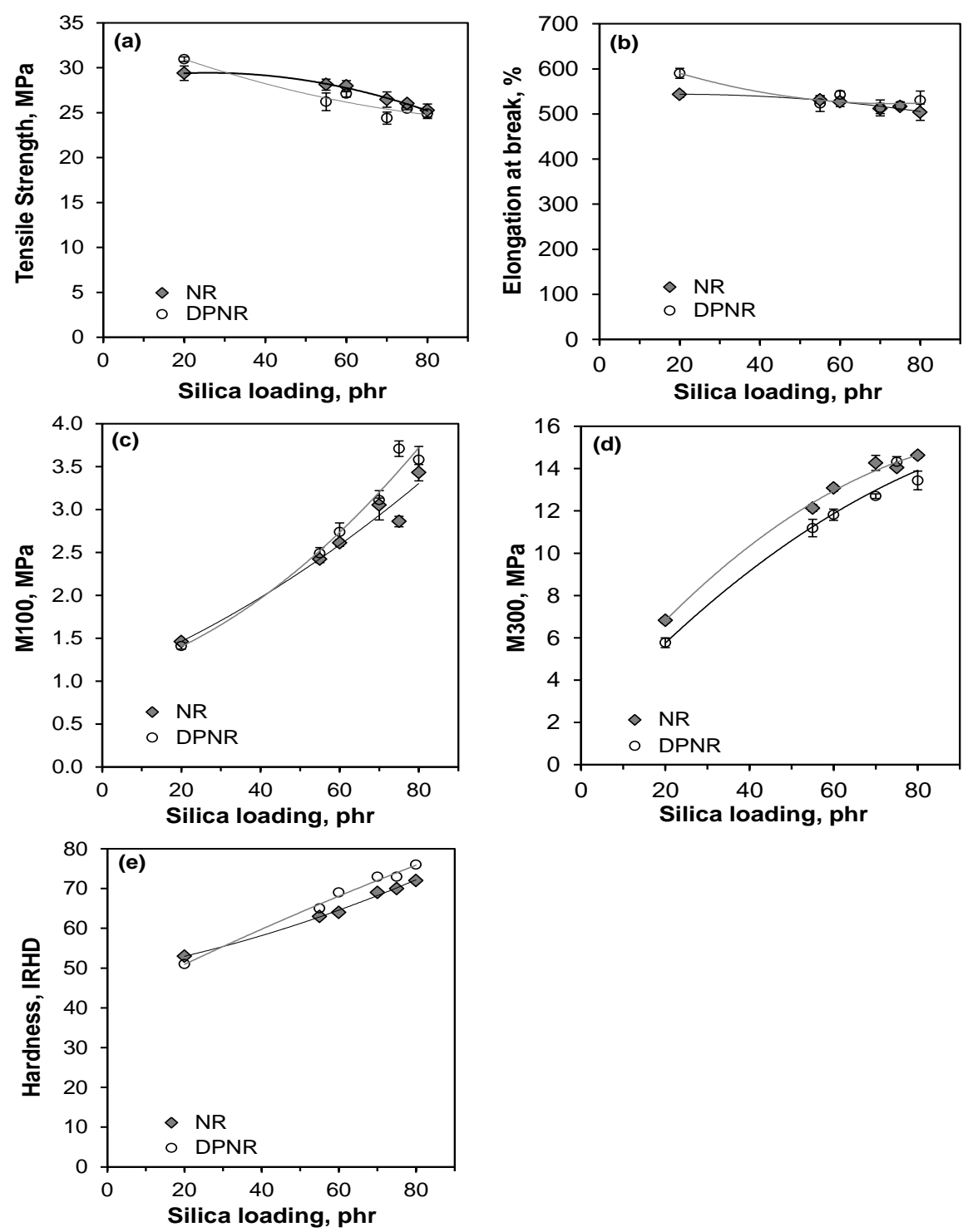

Figure 8.16 Comparison of mechanical properties of NR and DPNR vulcanizates as a function of filler loading; (a): Tensile strength, (b): Elongation at break, (c): M100, (d): M300, (e): Hardness. 
In Figure 8.17, the reinforcing index or ratio of M300/M100 of modified-silica vulcanizates shows a slight reduction above $70 \mathrm{phr}$ silica loading. On the other hand, the increase in tan delta at $60^{\circ} \mathrm{C}$ for both NR and DPNR vulcanizates is proportional to the amount of modified-silica. By increasing the amount of modified-silica, the filler-filler interaction is higher and in turn the filler-to-rubber interactions are lowered and thus give a higher tan delta at $60^{\circ} \mathrm{C}$.

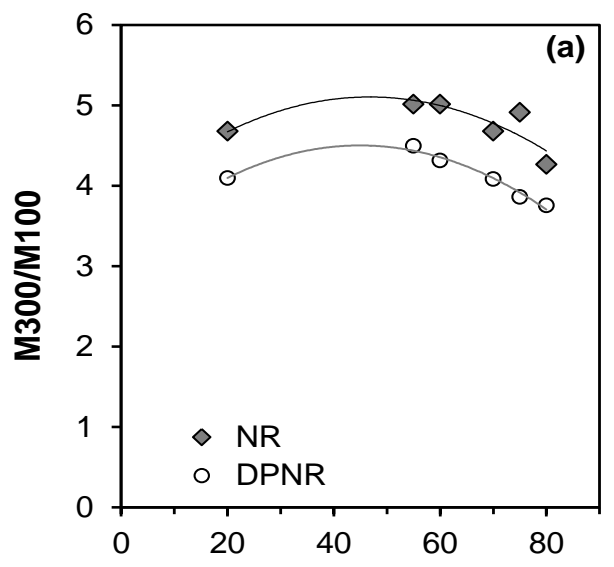

Silica loading, phr

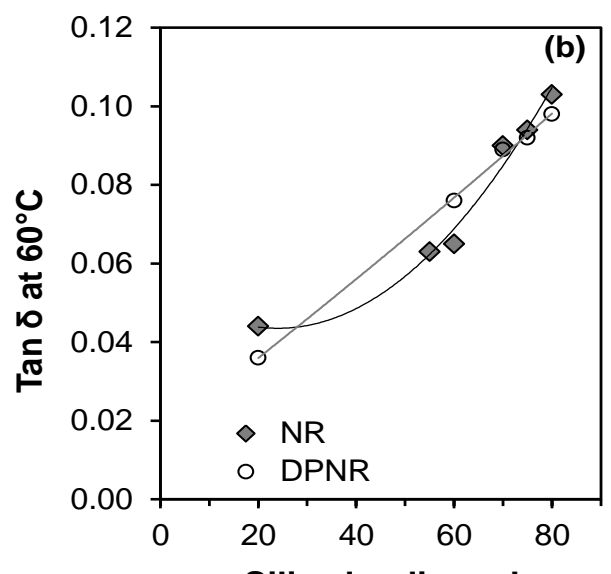

Silica loading, phr

Figure 8.17 Comparison of (a): M300/M100 ratio, and (b): tan delta at $60^{\circ} \mathrm{C}$ of modifiedsilica filled NR and DPNR vulcanizates.

\subsubsection{Comparison of modified-silica with in-situ TESPT-treated silica}

Figure 8.18 shows the filler-to-filler and filler-to-rubber interactions of compounds filled with modified-silica and in-situ TESPT-treated silica. The comparison is made between the 60 phr of silica Agilon ${ }^{\mathrm{TM}} 400$ compound and 55phr of the un-modified silica / 5phr of TESPT compound. For both NR and DPNR systems, the Payne effects of compounds with modified-silica are lower than those with TESPT-treated. This was expected as the surface of silica Agilon ${ }^{\mathrm{TM}} 400$ is already modified with a mixture of coupling and non-coupling agents which increases its hydrophobicity and results in a reduced silica-silica network. For TESPTtreated silica, the hydrophobation depends on the silanization degree during mixing. The TESPT-treated silica compounds have a higher chemically bound rubber content as compared to the modified-silica compounds. In the case of DPNR-silica Agilon, there is even no chemically bound rubber obtained at 60phr loading, only physically bound rubber is formed: Figure 8.12, indicating a weak rubber-to-filler interaction. 

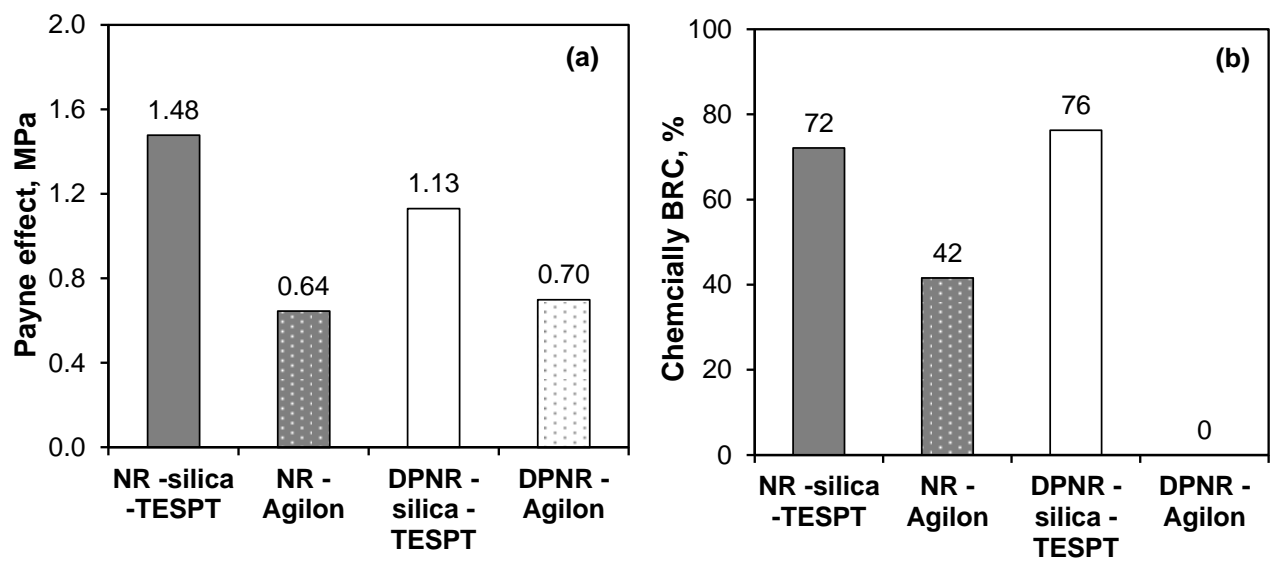

Figure 8.18Comparison of the Payne effects and chemically bound rubber contents of modified-silica with TESPT-treated silica compounds.

(a)

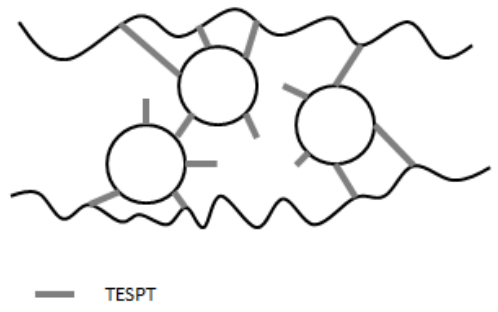

(b)

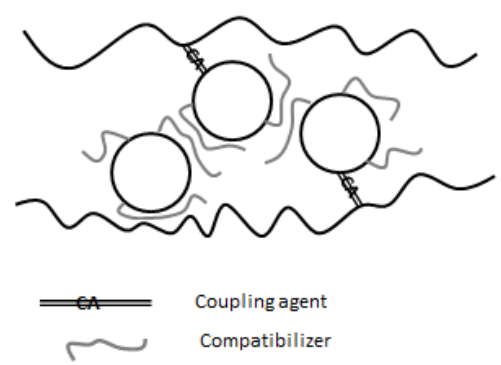

Figure 8.19 Schematic illustration of the difference of filler-filler and filler-to-rubber interactions between (a): TESPT-treated silica, and (b): modified-silica compounds.

The use of TESPT-treated silica and modified-silica reduces the filler-filler interactions in NR compounds. However, the mechanisms of the reduction of silica-silica interactions in both cases are different due to the surface modification with different coupling agents. In Figure 8.19(a), the silica surface is covered with TESPT and this prevents the silica-silica interaction and results in lower Payne effect. The sulfur moiety in TESPT creates silica-TESPT-rubber bonds and this results in strong filler-to-rubber interactions, as also reflected in the high chemically bound rubber content. The filler-filler interactions in the modified-silica compounds are lower than in the TESPT-treated silica compounds as the 
surface of modified-silica is covered with both coupling agent and compatibilizer or noncoupling agent, which prevent flocculation of the silica as pictured in Figure $8.19(\mathrm{~b})$. However, the non-coupling agent on the modified-silica surface will only increase the hydrophobicity of the silica, but does not contribute to silica-rubber coupling as the coupling agent does. The coupling agent on the surface of modified-silica, in this case the mercaptofunctionality, will lead to filler-to-rubber interaction as demonstrated by the results of the chemically bound rubber, however the amount of coupling agent on the silica surface is lower as compared to TESPT-treated silica. This explains the lower chemically bound rubber for modified-silica as compared to TESPT-treated silica. Why the chemically bound rubber content for the modified-silica DPNR combination at low loadings is zero is for the moment not clear.

The reinforcement index, M300/M100 of modified-silica vulcanizates, only shows a slight reduction as compared to TESPT-treated silica vulcanizates. This is observed for both NR and DPNR systems as shown in Figure 8.20. The tan delta at $60^{\circ} \mathrm{C}$, an indication of rolling resistance of tire tread compounds, for DPNR vulcanizates shows no difference between modified-silica and TESPT-treated silica. However, for the NR vulcanizate the use of modified-silica gives an improved reduction in the tan delta at $60^{\circ} \mathrm{C}$ as shown in Figure 8.20 .
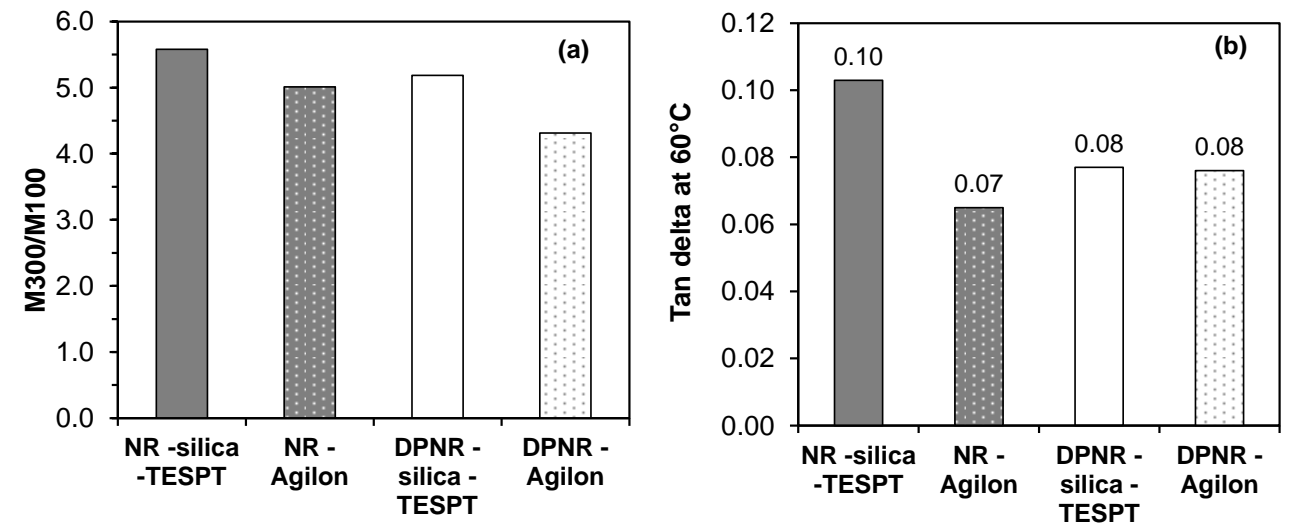

Figure 8.20 Comparison of the reinforcement index M300/M100 and tan delta at $60^{\circ} \mathrm{C}$ of modified-silica with TESPT-treated silica compounds. 


\subsection{CONCLUSIONS}

The reinforcement of pre-treated or surface-modified silica in NR and Deproteinized NR is investigated. The use of surface-modified silica eliminates the need for coupling agent during mixing. The commercial surface-modified precipitated silica, Agilon ${ }^{\mathrm{TM}} 400$ Performance silica, from PPG is a mercaptoorganometallic and non-coupling agent treated silica. The structure of aggregates of silica Agilon ${ }^{\mathrm{TM}} 400$ is similar to carbon black and highdispersible silica. The modified-silica is easily incorporated in NR during mixing as the silica surface is already silanized and has higher hydrophobicity which enhances its compatibility with NR. The use of silica Agilon ${ }^{\mathrm{TM}} 400$ improves the macro-dispersion of silica in NR and prevents silica flocculation especially at high silica loadings. Increasing the silica Agilon $^{\mathrm{TM}} 400$ loading in both NR and DPNR compounds increases the maximum torque of the vulcanization curve, Mooney viscosity and Payne effect. By using ATR-FTIR spectroscopy, the amount of silica in the compound can be monitored by the location of the peak absorbance in the silica region between $1250-1000 \mathrm{~cm}^{-1}$. The peaks of silica absorbance in both NR and DPNR vulcanizates are shown to shift to a lower wavenumber with increasing silica Agilon 400 loading. For an increase of silica Agilon 400 of 10 phr, the wavenumber of the peak of the silica region is decreased by $3.5 \mathrm{~cm}^{-1}$. The results show that there is no major difference in mechanical properties between NR and DPNR vulcanizates filled with modified-silica. The tensile strength, elongation at break and reinforcement index M300/M100 ratio, decrease with increasing silica loading. The increase in tan delta at $60^{\circ} \mathrm{C}$ and hysteresis energy loss for both NR and DPNR vulcanizates is proportional to the amount of silica Agilon ${ }^{\mathrm{TM}} 400$ used. This indicates the effect of the amount of silica Agilon $^{\mathrm{TM}} 400$ on rolling resistance and heat build-up of tread compounds. In comparison with in-situ TESPT treated silica, the pre-treated silica shows a significant improvement in tan delta at $60^{\circ} \mathrm{C}$ for $\mathrm{NR}$, although for DPNR no difference is seen.

\subsection{RefERENCES}

1. J.W.M. Noordermeer and W.K. Dierkes, in "Rubber Technologist's Handbook", Vol.2, J. White, S.K. De and K. Naskar, eds., Smithers Rapra Technology, Shawbury, Shrewsbury, Shropshire, UK, Chapter 3 (2008).

2. W. Meon, A. Blume, H-D. Luginsland, and S. Uhrlandt, in Rubber Compounding: Chemistry and Applications, B. Rodgers, Ed., Marcel Dekker: New York, Chapter 7 (2004).

3. L.A.E.M. Reuvekamp, J.W. ten Brinke, P.J. van Swaaij and J.W.M. Noordermeer, Rubber Chem. Technol., 75, 187 (2002). 
4. W.K. Kaewsakul, K. Sahakaro, W.K. Dierkes and J.W.M. Noordermeer, Rubber Chem. Technol., 85, 277 (2012).

5. S.S. Sarkawi, W.K. Dierkes and J.W.M. Noordermeer, Kautsch. Gummi Kunstst. 66(3), 27 (2013).

6. J.J. Martin, T.A. Okel, and R. Kollah, Fall 180th Technical Meeting, ACS Rubber Division, Cleveland, USA, paper no.70 (Oct. 2011).

7. T.A. Okel, Fall 180th Technical Meeting, ACS Rubber Division, Cleveland, USA, paper no.69 (Oct. 2011).

8. T.A. Okel, US Patent 6,342,560, to PPG Industries, Ohio (January 29, 2002).

9. D.F. Bergstrom, L.M. Boswell, M. D. Fisher, J. R. Hahn, T.A. Okel, and C.C. Reese, US Patent 6,384,125, to Dow Corning Corporation (May 7, 2002).

10. T.A. Okel, US Patent 6,649,684, to PPG Industries, Ohio (November 18, 2003).

11. T.A. Okel, Rubber World, 242(5), 13 (2010).

12. T.A. Okel, Rubber World, 244(6), 30 (2011).

13. T.A. Okel, Tire Technology International 2012, $42-45$ (2012).

14. M.L. Kralevich and J.L. Koenig, Rubber Chem. Technol., 71, 300 (1998).

15. S.S. Sarkawi, W.K. Dierkes and J.W.M. Noordermeer, Rubber World, 247(2), 26 (2012).

16. A.R.Payne, J.Polymer Sci.,Symposia. 48, 169 (1974). 


\section{Chapter 9}

\section{Dynamic and Mechanical Properties of Silica Reinforced NR/IR and NR/BR Blends}

Tire performance is related to the dynamic mechanical properties of tread materials. The choice of the rubber polymer with the optimum glass transition temperature, $\mathrm{Tg}$, or the proper combination of a blend polymers, play a key role in achieving a compromise between the many tire requirements. Natural Rubber (NR) provides low rolling resistance and low heat build-up properties but poor abrasion resistance. On the other hand, Polybutadiene Rubber (BR) provides an excellent abrasion resistance and optimum low rolling resistance, but inadequate skid resistance. The present chapter focuses on improving the reinforcement of the silica-silane system by blending NR with $10 \%$ vinyl-BR and also NR with $10 \%$ vinylpolyisoprene rubber (IR) at several different ratios. The skid resistance of the NR/IR blends is clearly superior as indicated by a higher tan delta at 0 to $30^{\circ} \mathrm{C}$. The NR/BR blends exhibit superior abrasion resistance to NR and good wet skid resistance, in addition to suitability for low temperature or winter performance. However, both NR/IR and NR/BR blends have a higher tan delta value at $30-70^{\circ} \mathrm{C}$ which indicates no improvement in the rolling resistance. A 80/10/10 of NR/BR/IR blend has a higher $T g$ than that of $N R$, and clear superiority for wet skid resistance and braking performance. However, the NR/IR/BR blend still exhibits unfavorable rolling resistance and heat build-up properties. 


\subsection{INTRODUCTION}

Tire performance is related to the dynamic mechanical properties of the materials used for its construction ${ }^{1}$. Rubber is a viscoelastic material. As rubber is deformed, a fraction of the energy is elastically stored and the rest of the energy is dissipated as heat, defined as hysteresis loss. The hysteresis losses within a tire, in combination with aerodynamic drag and friction in the contact path and with the rim, contribute to the total drag force on a moving vehicle ${ }^{2}$.

The phase angle, commonly expressed as the loss tangent $\tan \delta$, is the delay of stress response during sinusoidal deformation and can be taken as a parameter for the viscoelastic behavior of rubber ${ }^{3}$. The $\tan \delta$ is represented as follows:

$$
\tan \delta=\frac{G^{\prime \prime}}{G^{\prime}}
$$

Where G" is the loss modulus corresponding to the viscous behavior, and 'G' is the shear or storage modulus corresponding to the elastic behavior of the rubber. Both the storage modulus, G' and loss modulus, G" depend on frequency and temperature. The typical temperature- and frequency-dependence of these moduli for a viscoelastic material and their correlation with dynamic tire properties is depicted in Figure $9.1^{1,4}$.

(a)

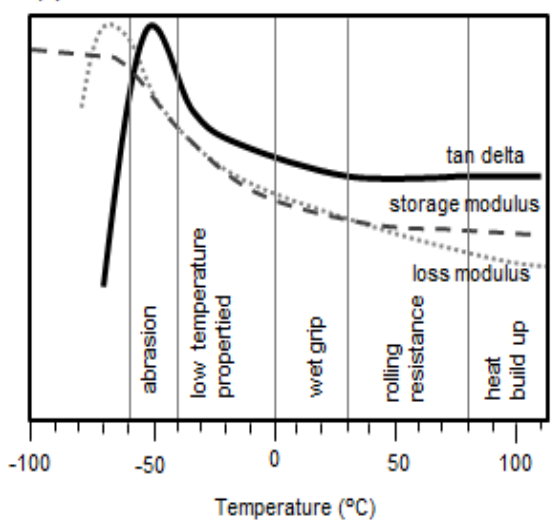

(b)

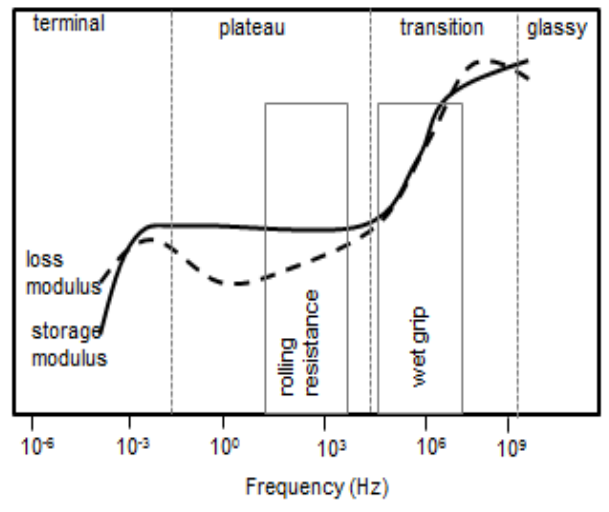

Figure 9.1 The temperature- and frequency-dependence of storage modulus G', loss modulus $G$ " and phase angle tan $\delta$ for a typical viscoelastic polymer.

The $\tan \delta$ curve against temperature in Figure 9.1(a) can be used to predict the important tire tread rubber properties. The glass transition temperature of the rubber gives an indication of the low temperature performance of the tire, as it indicates the limit of elastic behavior. A correlation between the glass transition temperature and abrasion resistance is 172 
generally accepted in tire technology $y^{1,5}$. The temperature range from $0^{\circ} \mathrm{C}$ to $30^{\circ} \mathrm{C}$ correlates with the prevailing road temperature, and the $\tan \delta$ at this range gives an indication of the skid behavior and traction, particularly on wet roads. For high wet skid resistance of a tire a high loss angle at a temperature around $0^{\circ} \mathrm{C}$ is required. The range between $30^{\circ} \mathrm{C}$ and approximately $70^{\circ} \mathrm{C}$ comprises the running temperatures of a tire. Thus, the $\tan \delta$ in the temperature zone at this range correlates with the rolling resistance. The loss angle at $60^{\circ} \mathrm{C}$ should be low for low rolling resistance. With further increase in temperature, rubber compounds start to degrade and limit the driving safety. In this temperature region the loss angle indicates the heat build-up properties. Hence, the heat build-up should be low in order to restrain the temperature increase.

The frequency region of rolling resistance and wet skid is depicted in Figure 9.1(b). Rolling resistance is affected by a low frequency distortion which is the deflection of the tire as it revolves. The angular frequency of a rolling tire is within the rubbery zone. Thus, the rolling resistance is related to the loss tangent of rubber compounds at the low frequency region within the rubber state. It is generally accepted that a lower loss tangent at low frequency leads to lower rolling resistance. On the other hand, wet traction is related to the lost tangent at high frequency. In general, a higher loss tangent at high frequency leads to high wet traction. The position of wet skid in the transition region between the rubber and glassy state, again shows that the glass transition temperature has a strong influence on this property of a tire. Rubbers with a high glass transition temperature are preferable for high resistance to wet skid.

In addition to the polymers, the dynamic properties of rubber compounds are further strongly influenced by the fillers used. The effect of fillers on the dynamic properties depends on the particle size of the filler; its surface activity, and the amount of filler used in the rubber compounds. The difference between the influence of carbon black and silica on the temperature profiles of $\tan \delta$ for rubber compounds is depicted in Figure $9.2^{6}$. The main difference between silica-filled and carbon black-filled compounds is the phase angle at the higher temperature range; for silica-filled compounds it is lower, which means that the rolling resistance of tire treads based on silica-filled compounds is lower than for carbon black-filled compounds ${ }^{7}$. On the other hand, the loss tangent of silica-filled compounds at lower temperature is higher compared to that of a carbon black-filled compound, indicating that the wet traction of tire treads based on a silica compound is higher than that of a carbon blackfilled compound.

Both polymer macrostructure and microstructure are important in determining the rubber characteristics required to meet tire performance properties. 
Polymer macrostructure : molecular weight, crosslink distribution, chain branching, crystallite formation;

Polymer microstructure $\quad$ : the arrangement of the monomers within a polymer chains (molecular configurations, stereochemistry)

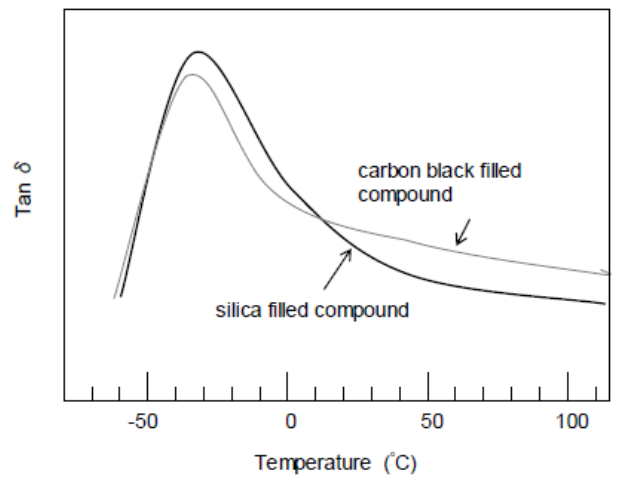

Figure 9.2 Temperature-dependence of phase angle for carbon black and silica filled rubber.

Butadiene rubber (BR) can adopt one of the three configurations as depicted in Figure 9.3. BR with high cis content (92\%) is more difficult to process at factory processing temperature but shows better abrasion resistance. On the other side, BR with low cis content tends to process easily. High-trans BR (93\% trans) tends to be tough, crystalline materials, whereas high-vinyl BR shows good wet skid and wet traction performance.

The current silica technology for tires makes use of a blend of high-vinyl solution-SBR and high-cis BR, filled with highly-dispersible silica and an effective silane coupling agent. Silanes make use of the large reactivity of thiol-groups (-SH) or - $\mathrm{S}$-S radicals towards unsaturated double bond $(\mathrm{C}=\mathrm{C})$ functionalities in the polymer. The most reactive group in the polymer is the pendant 1,2-vinyl. The amount of vinyl groups in high-vinyl solution-SBR can vary from 20 to $70 \%$. However, the glass transition temperature ( $\mathrm{Tg}$ ) of high-vinyl solution-SBR is too high. Hence a blend of high-vinyl solution-SBR with BR is necessary to meet the different requirements in tread properties.

In a blend compound, silica tends to transfer from an elastomer with low unsaturation, e.g. EPDM, to rubbers with high unsaturation such as SBR. It has been demonstrated, that preferential take-up of silica by various rubbers shows the affinity of silica to decrease in the following order ${ }^{8}$ :

NBR $>$ solution-SBR $>\mathrm{NR} \geq \mathrm{BR}>$ high-vinyl $\mathrm{BR}>\mathrm{EPDM}>\mathrm{IIR}$ 


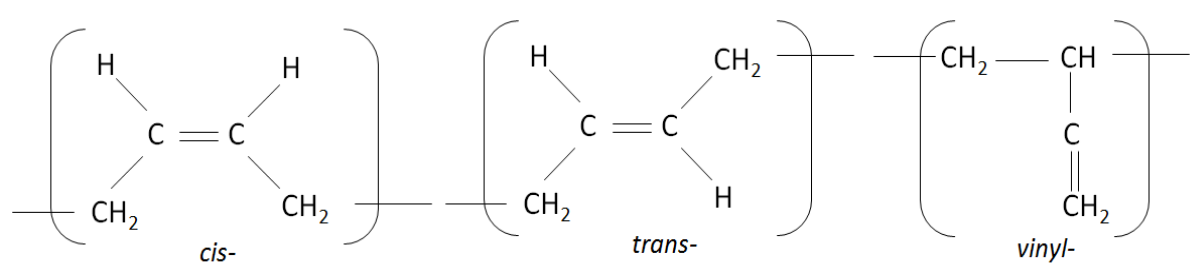

Figure 9.3 Polymer microstructure: possible configurations for butadiene in BR.

NR exhibits good low rolling resistance and low heat build-up properties but poor abrasion resistance ${ }^{1,5}$. On the other hand, BR provides an excellent abrasion resistance, optimum low rolling resistance and minimum heat build-up, but inadequate skid resistance. A blend of NR with BR is a step to improve the abrasion resistance of a NR-based tread compound. In addition, blending of NR with vinyl-IR is to improve the reactivity of silicasilane-rubber coupling.

The present chapter focuses on the reinforcement of a silica-silane system in natural rubber (NR) using blends of NR/IR and NR/BR. Both IR and BR is selected to have $10 \%$ vinyl-content. The mechanical and dynamic properties of the NR/IR and NR/BR blends with different ratios are investigated.

\subsection{EXPERIMENTAL}

\subsubsection{Materials}

Standard Malaysian Natural Rubber (SMR 20) was provided by the Malaysian Rubber Board. Polyisoprene, Isogrip with $10 \%$ vinyl-content was supplied by Karbochem, South Africa. A medium cis-1,4-polybutadiene, Buna CB45 with 10\% vinyl content was provided by Lanxess, Germany. A comparison of the properties of the rubbers used is shown in Table 9.1. Silica Ultrasil 7005 and silane coupling agent, bis-(triethoxysilylpropyl) tetrasulfide (TESPT) were supplied by Evonik, Germany. Treated distillate aromatic extract (TDAE) oil was obtained from Hansen \& Rosenthal. Zinc oxide, stearic acid and sulfur were from Sigma Aldrich. Antioxidant TMQ, N-cyclohexyl-2-benzothiazyl sulfenamide (CBS) and diphenyl guanidine (DPG) were from Flexsys, the Netherlands. All ingredients were used as received.

The compound recipe was based on a truck tire tread composition: Rubber (100phr), silica (55phr), TESPT (5phr), TDAE oil (8phr), zinc oxide (2.5phr), stearic acid (1phr), TMQ (2phr), sulfur (1.4phr), CBS (1.7phr) and DPG (2phr). 
The ratios of NR/IR and NR/BR blends are shown in Tables 9.2 and 9.3, respectively. In addition, a tri-blend of NR/IR/BR was also prepared as shown in Table 9.4.

Table 9.1 Properties of rubbers used

\begin{tabular}{lccc}
\hline Rubber & NR & IR & BR \\
\hline Trade name & SMR 20 & Isogrip & Buna CB45 \\
Cis-1,4- content (\%) & $>99$ & 30 & 38 \\
Vinyl- content (\%) & - & 10 & 10 \\
$3,4-$ addition (\%) & - & 60 & - \\
ML(1+4),100 $\circ$ C & 100 & 76 & 45 \\
$\operatorname{Tg}(\mathrm{DSC})$ & -70 & -11 & -93 \\
\hline
\end{tabular}

Table 9.2 NR/IR blend ratios (phr)

\begin{tabular}{lcccccc}
\hline Rubber & 1 & 2 & 3 & 4 & 5 & 6 \\
\hline NR & 100 & 90 & 80 & 70 & 60 & 50 \\
IR & 0 & 10 & 20 & 30 & 40 & 50 \\
\hline
\end{tabular}

Table 9.3 NR/BR blend ratios (phr)

\begin{tabular}{lcccccc}
\hline Rubber & 7 & 8 & 9 & 10 & 11 & 12 \\
\hline NR & 100 & 90 & 80 & 70 & 60 & 50 \\
BR & 0 & 10 & 20 & 30 & 40 & 50 \\
\hline
\end{tabular}

Table 9.4 NR/IR/BR blend ratio (phr)

\begin{tabular}{ll}
\hline Rubber & 13 \\
\hline NR & 80 \\
IR & 10 \\
BR & 10 \\
\hline
\end{tabular}

\subsubsection{Compounding}

All ingredients except the curatives were mixed in an internal mixer, Brabender Plasticoder $350 \mathrm{~S}$ lab station. The mixing was done at $60 \mathrm{rpm}$ rotor speed, 0.7 fill factor, 14 minutes mixing time and $150^{\circ} \mathrm{C}$ dump temperature. After 24 hours, the curatives were added to the masterbatches on a two-roll mill. The compounds were mixed and vulcanized according to the experimental section of Chapter 3. 


\subsubsection{Sample analysis}

Mooney viscosity, vulcanization curves, Payne effect, bound rubber content, tensile properties and tan delta at $60^{\circ} \mathrm{C}$ of the compounds were investigated according to the methods as described in the experimental section in Chapter 3. The apparent crosslink density was determined according to the method as described in Chapter 4 .

\section{Dynamic properties}

The dynamic properties of vulcanizates: storage modulus, loss modulus and glass transition temperature were measured using a Metravib Dynamic Mechanical Analyzer (DMA) viscoanalyzer VA 2000. The samples were cut from the vulcanized sheets of $2 \mathrm{~mm}$ thickness. A temperature sweep measurement from -80 to $+80^{\circ} \mathrm{C}$ was performed in tension mode at a frequency of $10 \mathrm{~Hz}$ and dynamic strain of $0.1 \%$.

Fourier Transform Infrared (FTIR) Analysis

Infrared spectra of vulcanizate samples were recorded using a Fourier Transform Infrared (FTIR) Perkin Elmer 100 series system with a Attenuated Total Reflection (ATR) attachment. All spectra were collected at $4 \mathrm{~cm}^{-1}$ resolution, 10 scans per sample, scan range of $4000-650 \mathrm{~cm}^{-1}$, and subtracting the background at the beginning of the test. The spectra were analyzed using the Spectrum100 software without correction.

\subsection{RESULTS AND DISCUSSION}

\subsubsection{Influence of NR/IR blends on silane reactivity}

Since both NR and IR have the same polyisoprene structure, blending the two polymers is expected to result in a good compatibility. By increasing the ratio of IR in the blend, the amount of vinyl-groups in the compound is raised and it is postulated that the reactivity of the silane coupling agent, TESPT, towards natural rubber will be improved.

The filler-filler interactions of the NR/IR blends depend on the amount of the IR ratio in the blends as shown in Figure 9.4(a). The Payne effect of the NR/IR blend compounds shows an increase with increment of the IR ratio in the blends. It seems that silica-silica interactions become stronger when more vinyl content (as contained in the IR) is added in the blend. One possible explanation is a preferential location of silica in the NR phase rather than in the IR phase, which results in more silica present in the decreasing NR phase when the IR ratio is increased, and hence a higher Payne effect is observed. In the case of the NR/IR blend, the affinity of silica can be expressed as follows:

$$
\mathrm{NR}>\text { vinyl-IR }
$$

The filler-filler interactions in turn affect the processability of the blends due to the hydrodynamic effect. There is a good correlation between the increase in the Mooney 
viscosity and the Payne effect as shown in Figure 9.4(b). The Mooney viscosity of the NR/IR blends increases with higher filler-filler interactions, which relates to the hydrodynamic effect of the filler. Nonetheless, the complex viscosity of the NR/IR blends shows little difference between the varying ratios of the blend as shown in Figure 9.5. It is noticed that the 50/50 $\mathrm{NR} / \mathrm{IR}$ blend has only slightly higher complex viscosity than NR.
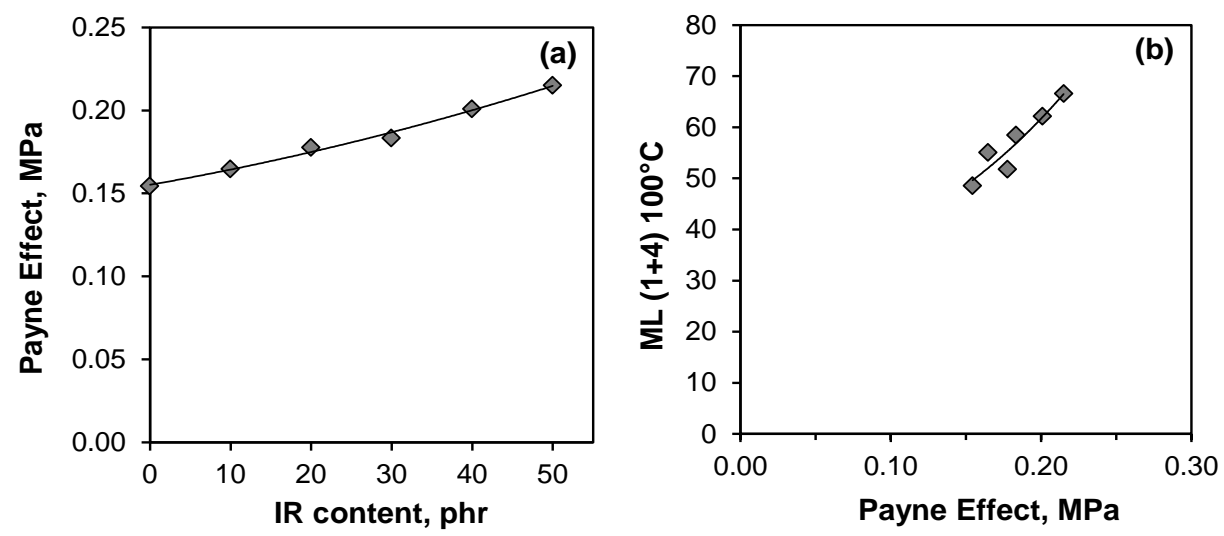

Figure 9.4 (a) The effect of IR content on Payne effect of NR/IR blends; and (b) correlation between Mooney viscosity and Payne effect of NR/IR blends.

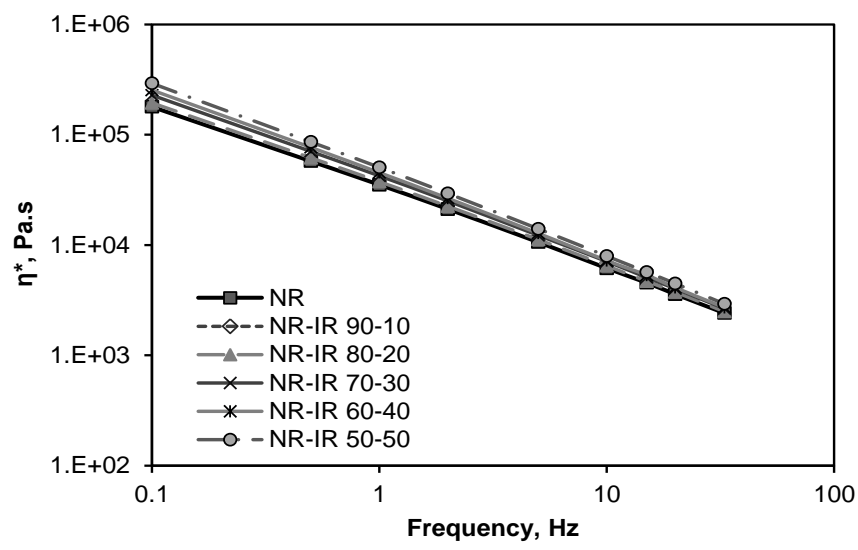

Figure 9.5 Complex viscosity of NR/IR blends as a function of frequency.

The chemically bound rubber of NR/IR blends are comparable to NR as shown in Figure 9.6(a). It is noticed that the chemically bound rubber content is not influenced by the IR ratio in the NR/IR blend, while the physically bound rubber shows a slight decrease with increasing IR ratio. The chemically bound rubber results indicate that no clear silane to 
rubber coupling occurs before vulcanization. Figure 9.6(b) shows the Payne effect of the NR/IR blends after vulcanization. The Payne effect after vulcanization can be used as indicator of silane reactivity towards rubber. With increasing IR with $10 \%$-vinyl content in the blend, the filler-filler interaction is reduced after vulcanization and this demonstrates the silica-to-rubber coupling in the vulcanized blend.
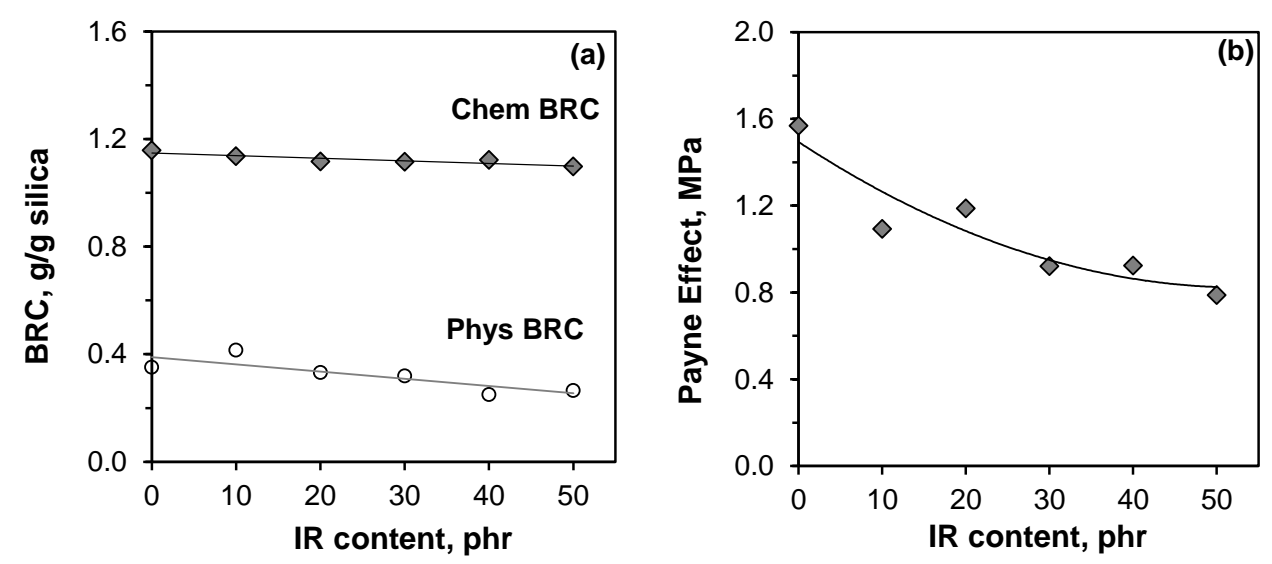

Figure 9.6 (a): Chemically and physically bound rubber contents of NR/IR blends;

(b) Payne effect of NR/IR blends after vulcanization.

\subsubsection{Dynamic and mechanical properties of NR/IR blends}

The dynamic properties of vulcanized NR/IR blends at varying ratio are shown in Figures 9.7 and 9.8. The effect of the variation of the blend ratio can be clearly seen in the change of tan delta peak or glass transition temperature, $\mathrm{Tg}$, of the NR/IR blends. Due to the higher $\mathrm{Tg}$ of vinyl-IR as compared to NR, increasing the IR content in the blends results in a shift of the $\mathrm{Tg}$ of the blends as shown in Figure 9.9. The shift of the $\mathrm{Tg}$ can be correlated to the Fox equation ${ }^{9}$ based on composition of the polymers in the blend. This indicates that increasing the IR ratio content reduces the low temperature flexibility and the abrasion resistance of the blends. On the other hand, the skid resistance of the NR/IR blends is clearly superior over the NR vulcanizates because of the higher $\tan \delta$ in the range from 0 to $30^{\circ} \mathrm{C}$ :Table 9.5 . It is expected that the NR/IR blends give poor rolling resistance as the addition of IR with $10 \%$ vinyl groups basically shifts the Tg curve towards higher temperature, and hence a higher $\tan \delta$ value at the range of 30 to $70^{\circ} \mathrm{C}$ is also observed. In addition, broadening of the peak of $\tan \delta$ is clearly seen as the ratio of vinyl-IR is increased in the blends. The storage modulus, E' of the NR/IR blends also shows a shorter rubbery region with increasing IR ratio. 


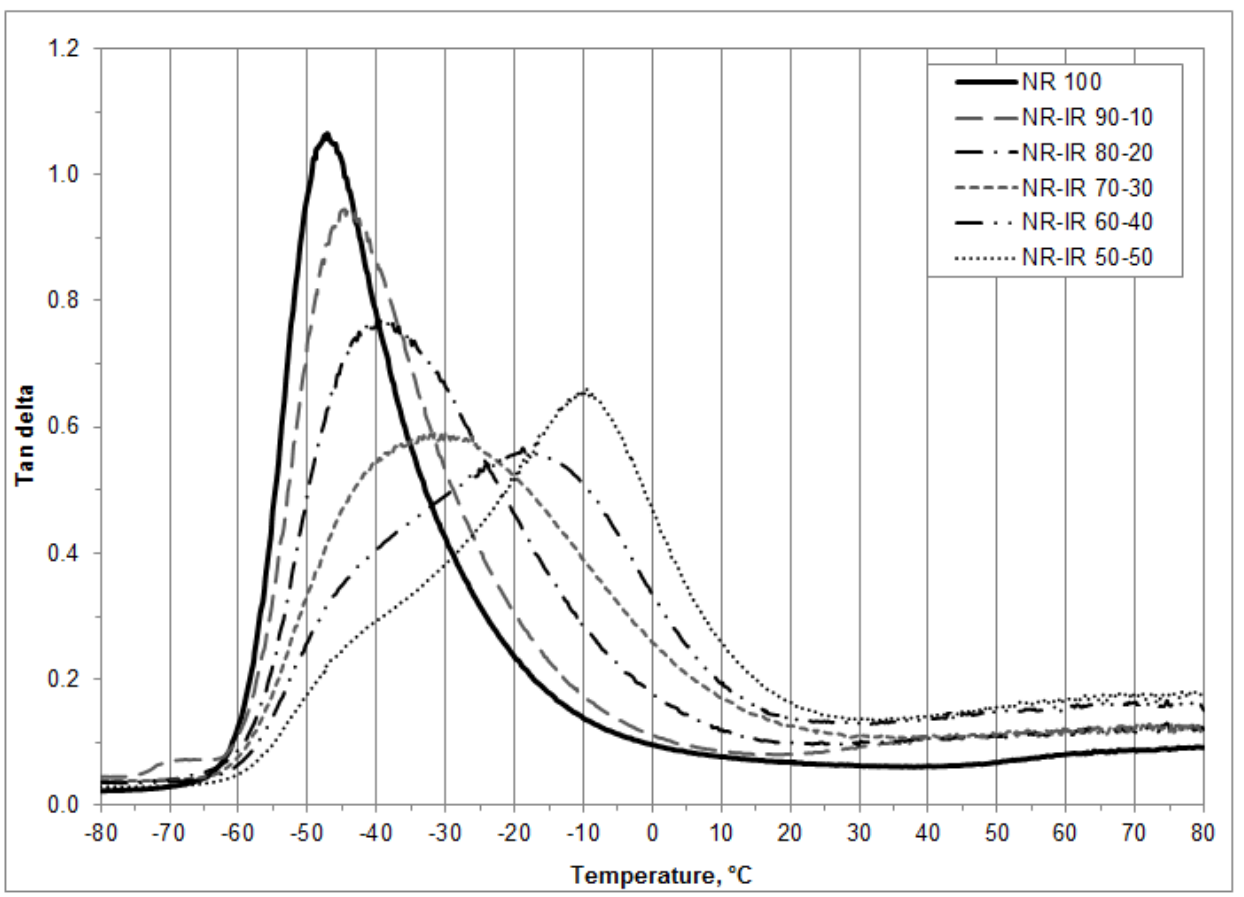

Figure 9.7 Effect of blend ratio on the tan delta of the NR/IR blends. DMA measurement at $10 \mathrm{~Hz}$.

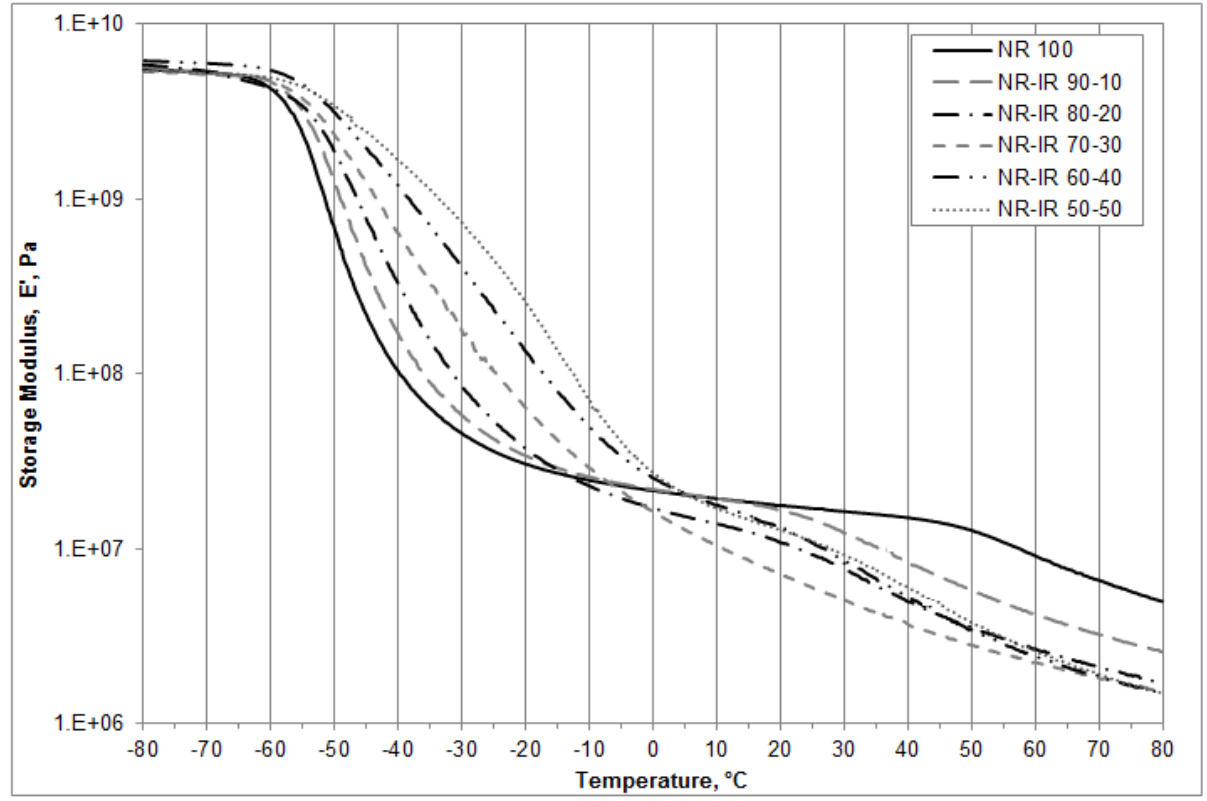

Figure 9.8 Effect of blend ratio on the storage modulus, E' of NR/IR blends. 


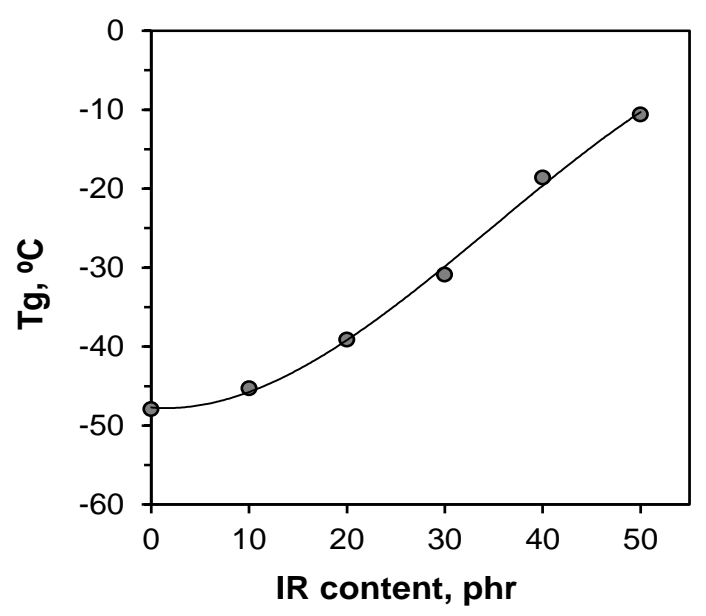

Figure 9.9 Glass transition temperature, $\mathrm{Tg}$ of $\mathrm{NR} / \mathrm{IR}$ blends as a function of IR ratio.

Table 9.5 Comparison of glass transition temperature, tan delta at $0^{\circ} \mathrm{C}$ and $60^{\circ} \mathrm{C}$ of $\mathrm{NR} / \mathrm{IR}$ blends at different ratios (DMA 10Hz).

\begin{tabular}{lccc}
\hline & $\operatorname{Tg}\left(^{\circ} \mathrm{C}\right)$ & Tan delta at $0^{\circ} \mathrm{C}$ & Tan delta at $60^{\circ} \mathrm{C}$ \\
\hline NR & -48 & 0.10 & 0.08 \\
NR/IR 90/10 & -45 & 0.11 & 0.12 \\
NR/IR 80/20 & -39 & 0.18 & 0.12 \\
NR/IR 70/30 & -31 & 0.27 & 0.12 \\
NR/IR 60/40 & -19 & 0.34 & 0.15 \\
NR/IR 50/50 & -11 & 0.48 & 0.16 \\
\hline
\end{tabular}

The stress-strain curves of the NR/IR blends are shown in Figure 9.10(a). It is clear that changing the NR/IR blend ratio leads to a significant change in the stress-strain properties, where a decrease in stress is observed with increase in $10 \%$ vinyl-IR ratio in the blends. The tensile strength shows a clear reduction when more IR ratio is added in the blend as shown in Figure 9.11. This is expected as the strain-induced crystallization will be lowered with the amount of vinyl-IR introduced in the NR/IR blends. Moreover, the drop in the storage modulus for NR/IR blends starts at a temperature of $25^{\circ} \mathrm{C}$ as compared to NR at above $50^{\circ} \mathrm{C}$ : Figure 9.8. In addition it also indicates the preference of silica to be in the NR phase, which results in the decrease of tensile strength as the amount of filler in NR is increased. However, the elongation at break is not much affected with the increase of IR ratio in the blends. The modulus at $100 \%$ shows no influence at the variation of blend ratio. 
In contrast, the M300 exhibits a significant reduction with increasing IR ratio content in the blends. The reduction in tensile strength and $M 300$ of $N R / I R$ blends also relates to a decrease in crosslink density when vinyl groups are incorporated in the blend. In Figure 9.10(b), the apparent crosslink density of the blends decreases with the increasing IR content above $20 \mathrm{phr}$. In order to maintain a comparable crosslink density to the NR compound, blends of $90 / 10$ and $80 / 20$ are advisable.
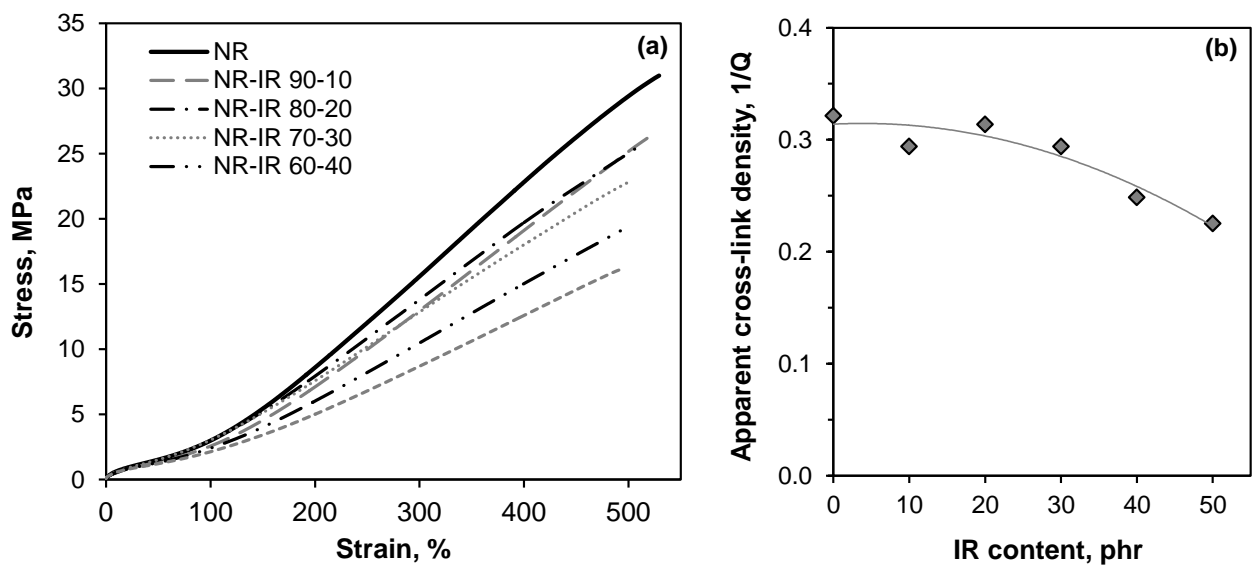

Figure 9.10 (a) Stress-strain curves of NR/IR blends; (b) Apparent crosslink density (1/Q) of $N R / I R$ blends as a function of increasing IR ratio content.

(a)

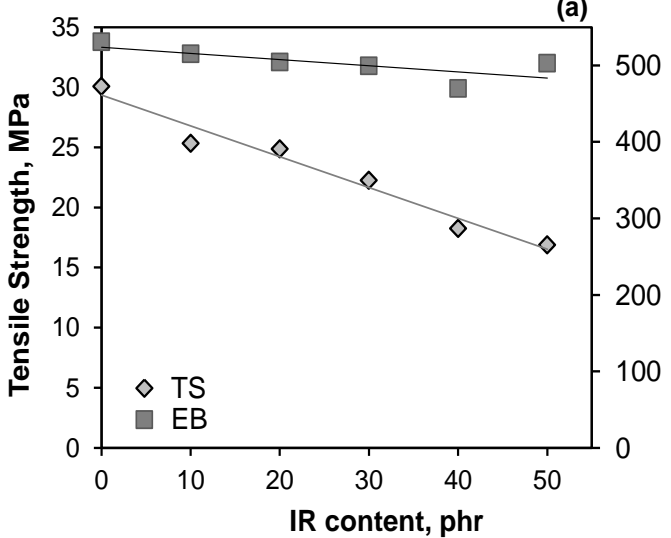

(b)

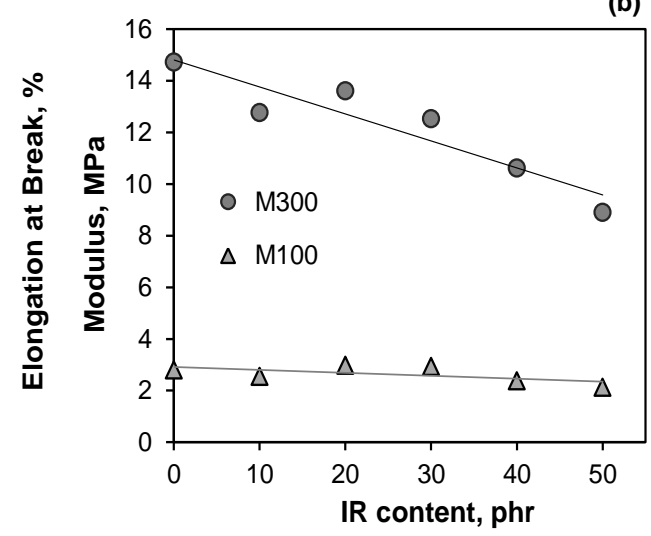

Figure 9.11 Tensile properties of NR/IR blends: (a): Tensile strength and Elongation at break; (b): Modulus at $100 \%$ and $300 \%$. 


\subsubsection{Influence of NR/BR Blends on Silane Reactivity}

The influence of the BR ratio in the NR/BR blends on the filler-filler interactions is shown in Figure 9.12(a). The Payne effect of the NR/BR blend compounds again shows a slight increase at higher $\mathrm{BR}$ ratio. With higher amount of vinyl content via the BR present in the blend, the filler-filler interaction also increases. As observed with the NR/IR blends, the fillerfiller interaction also influences the Mooney viscosity of the NR/BR blends. As demonstrated in Figure 9.12(b), there is reasonable correlation between viscosity and Payne effect for the NR/BR blends. Figure 9.13 shows the complex viscosity of the NR/BR blend compounds. The compounds have generally higher viscosities than the pure NR. The behavior of the complex viscosity is comparable in the blends and shown to increase steadily with the amount of vinyl content via the BR.
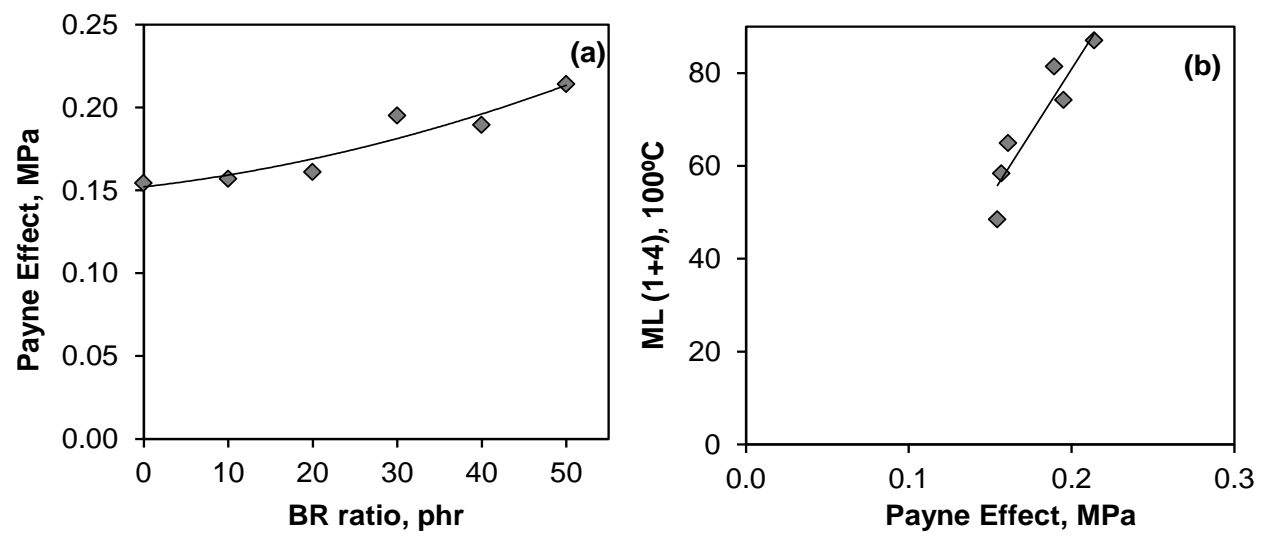

Figure 9.12 (a) The effect of BR ratio on Payne effect of NR/BR blends; and (b) correlation between Mooney viscosity and Payne effect of NR/BR blends

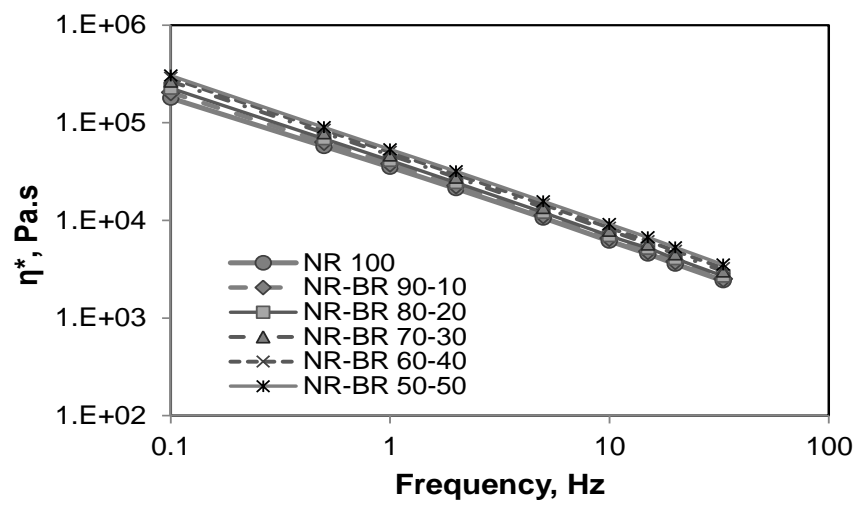

Figure 9.13 Complex viscosity of NR/BR blends as a function of frequency sweep. 
Figure 9.14(a) shows the chemically and physically bound rubber of the NR/BR blends. The chemically bound rubber only shows a very slight decrease with incorporation of $10 \%$ vinyl$B R$ in the NR/BR blends. In addition, no influence of BR ratio on physically bound rubber is observed. The sulfur moiety of the silane coupling agent will react with rubber and also the vinyl groups of BR during vulcanization. The Payne effect after vulcanization is reduced with increasing 10\%-vinyl BR content in the blend. As compared to the pure NR compound, the filler-filler interaction after vulcanization is lower for the blends, as shown in Figure 9.14(b). Incorporation of the vinyl group of BR in the blends helps to improve the silica-to-rubber coupling and this results in the lower Payne effect for the NR/BR blends.
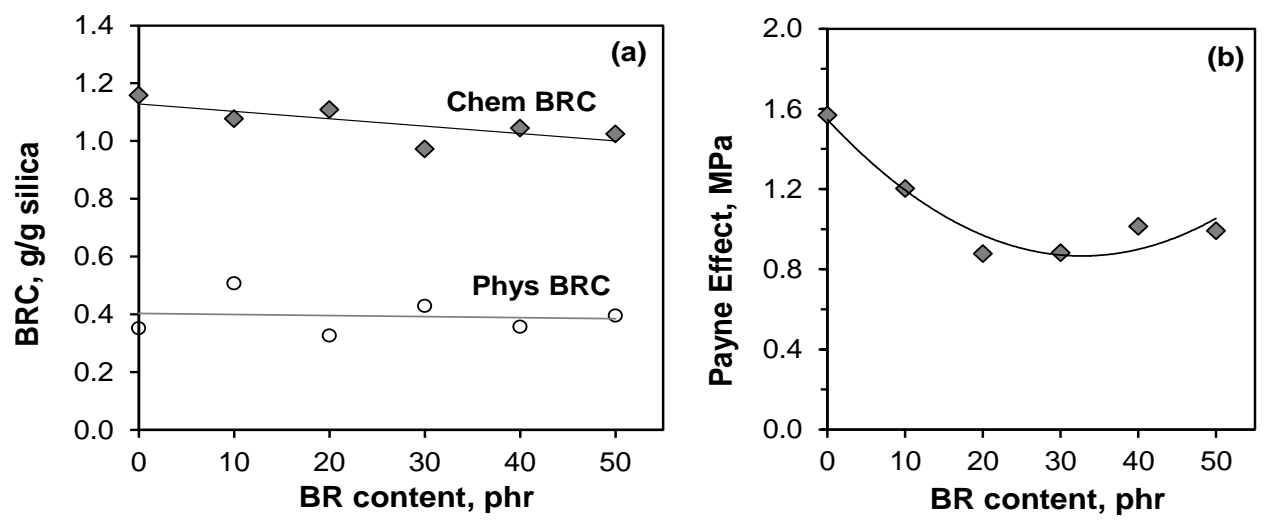

Figure 9.14 (a): Chemically and physically bound rubber content of NR/BR blends;

(b): Payne effect after vulcanization of NR/BR blends.

\subsubsection{Dynamic and Mechanical Properties of NR/BR Blends}

The effect of variation of the blend ratio on the $\mathrm{Tg}$ of the NR/BR blends is shown in Figure 9.15. The $\mathrm{Tg}$ of the NR/BR blends is shifted to lower temperature with increasing $B R$ content, due to the lower $\mathrm{Tg}$ of $\mathrm{BR}$ as compared to NR. This indicates that increasing the $\mathrm{BR}$ ratio improves the low temperature flexibility and the abrasion resistance of the blends. It is an excellent component for winter tires. On the other hand, the wet skid resistance at $0^{\circ} \mathrm{C}$ of the NR/BR blends is almost comparable to the pure NR vulcanizate. Only the 90/10 NR/BR blend shows a higher tan $\delta$ at $0^{\circ} \mathrm{C}$. However, it is noticed that for all NR/BR blends the tan $\delta$ in the range of $10-30 \stackrel{\circ}{C}$ is better than for NR, which indicates improvement in the skid resistance. The addition of $10 \%$-vinyl BR basically shifts the $\mathrm{Tg}$ curve towards lower temperature, and it would be expected that the tan $\delta$ in the rolling resistance region will be lowered as well. However, the slightly higher tan $\delta$ values observed at the range of $60^{\circ} \mathrm{C}$ :Table 9.6 , gives a clear indication of no improvement in the rolling resistance for the 
NR/BR blends. The storage modulus of the NR/BR blends is lower compared to NR, as shown in Figure 9.16. In addition, the rubbery plateau is also shorter for the NR/BR blends than for the NR vulcanizate, indicating reduced elasticity of the blends.

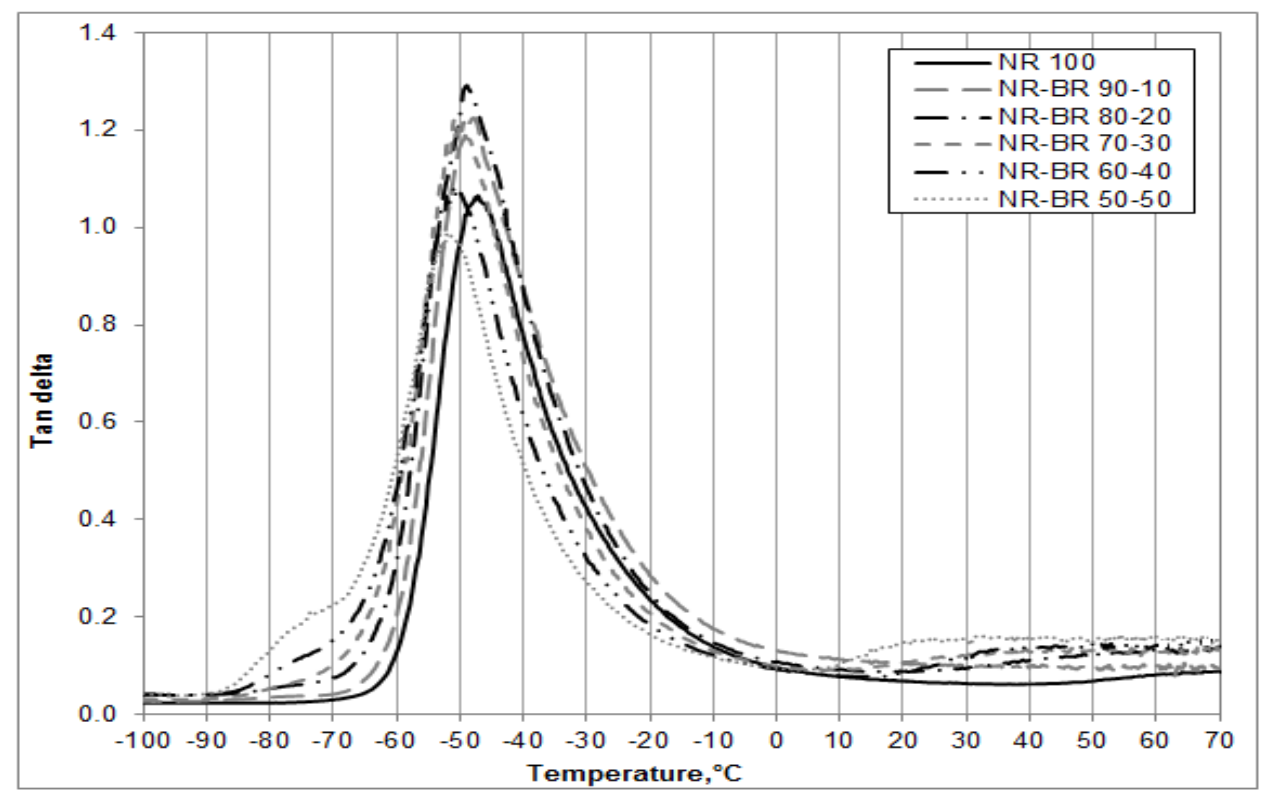

Figure 9.15 The effect of blend ratio on the $\mathrm{Tg}$ of the NR/BR blends

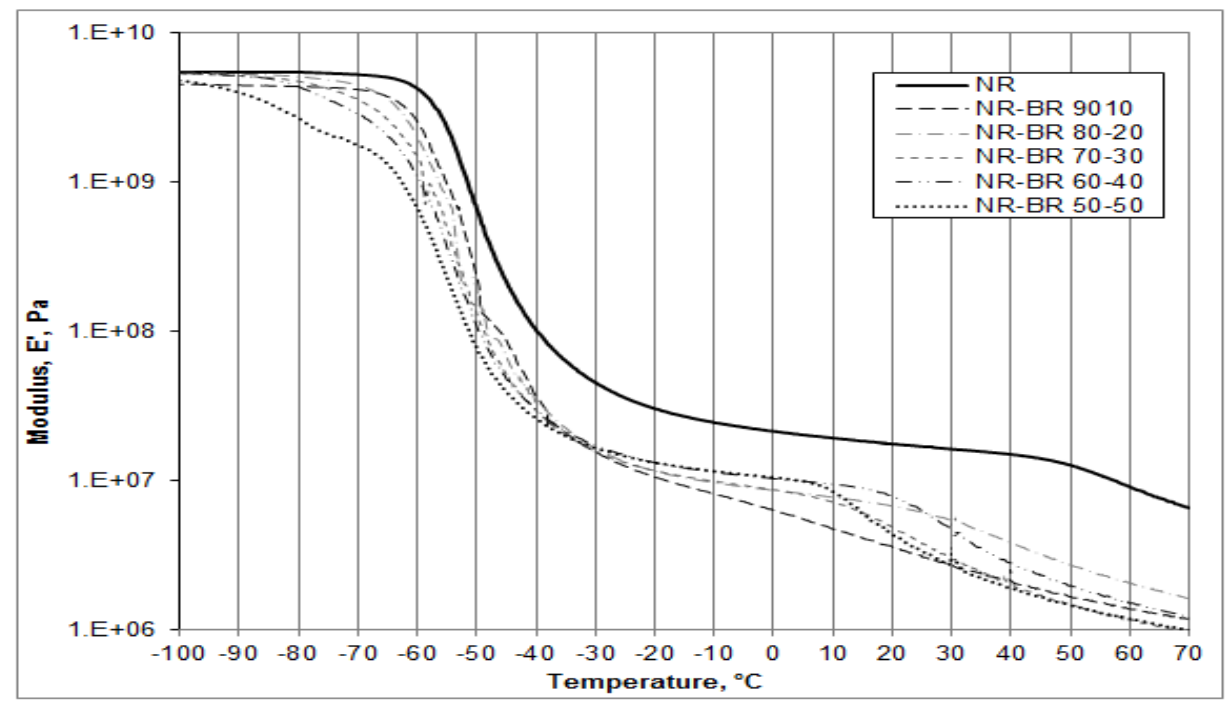

Figure 9.16 The effect of blend ratio on the storage modulus, E' of NR/BR blends. 
Table 9.6 Comparison of glass transition temperature, tan delta at $0^{\circ} \mathrm{C}$ and $60^{\circ} \mathrm{C}$ of $\mathrm{NR} / \mathrm{BR}$ blends at different ratios (DMA $10 \mathrm{~Hz})$.

\begin{tabular}{lccc}
\hline & $\operatorname{Tg}\left(^{\circ} \mathrm{C}\right)$ & Tan delta at $0^{\circ} \mathrm{C}$ & Tan delta at $60^{\circ} \mathrm{C}$ \\
\hline NR & -48 & 0.10 & 0.08 \\
NR/BR 90/10 & -48 & 0.13 & 0.10 \\
NR/BR 80/20 & -49 & 0.11 & 0.11 \\
NR/BR 70/30 & -51 & 0.10 & 0.13 \\
NR/BR 60/40 & -52 & 0.09 & 0.14 \\
NR/BR 50/50 & -53 & 0.09 & 0.16 \\
\hline
\end{tabular}

Figure 9.17(a) shows the stress-strain curves of NR/BR blends at different ratio. It is interesting to see that the NR/BR blends exhibit almost comparable stress-strain properties, only the stress level is affected slightly by the increase in BR ratio in the NR/BR blends. Both tensile strength and elongation at break of the NR/BR blends decrease linearly with increasing BR ratio as shown in Figure 9.18. In comparison with pure NR, the 50/50 NR/BR blend exhibits only about half of the tensile strength. However, M300 and M100 are not affected by the blend ratio. These relate to the stress-strain properties and also to the crosslink density. The apparent crosslink density of the blends shows no clear changes with the BR ratio as shown in Figure 9.17(b).
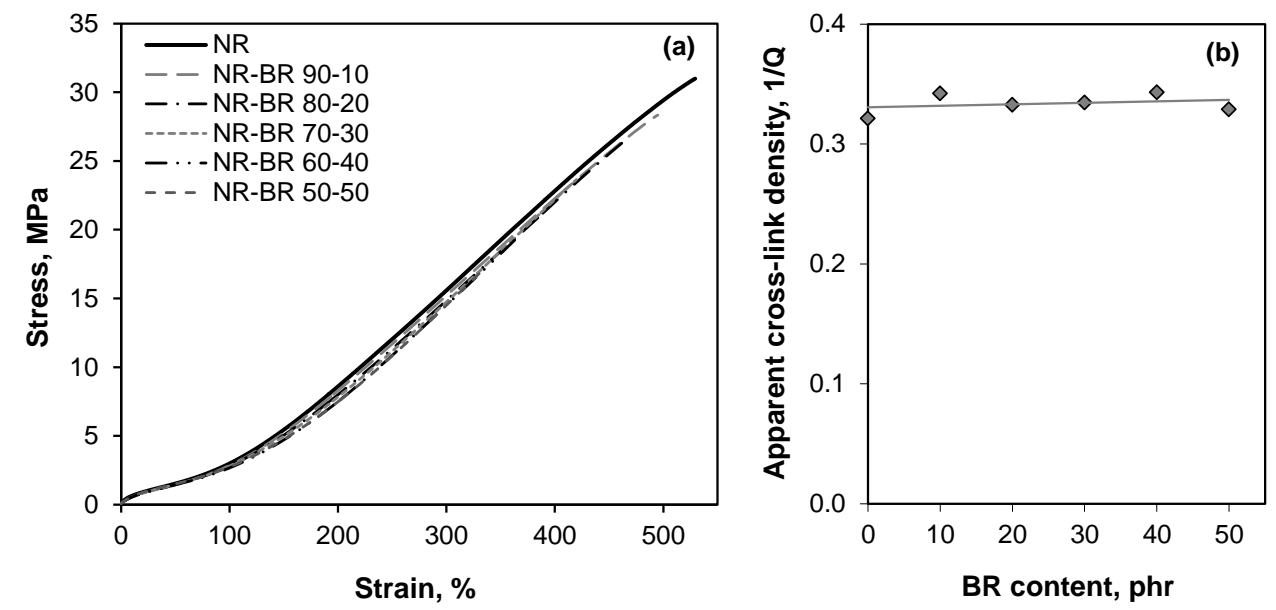

Figure 9.17 (a): Stress-strain curves of NR/BR blends; (b):Apparent crosslink density, 1/Q of $N R / B R$ blends. 

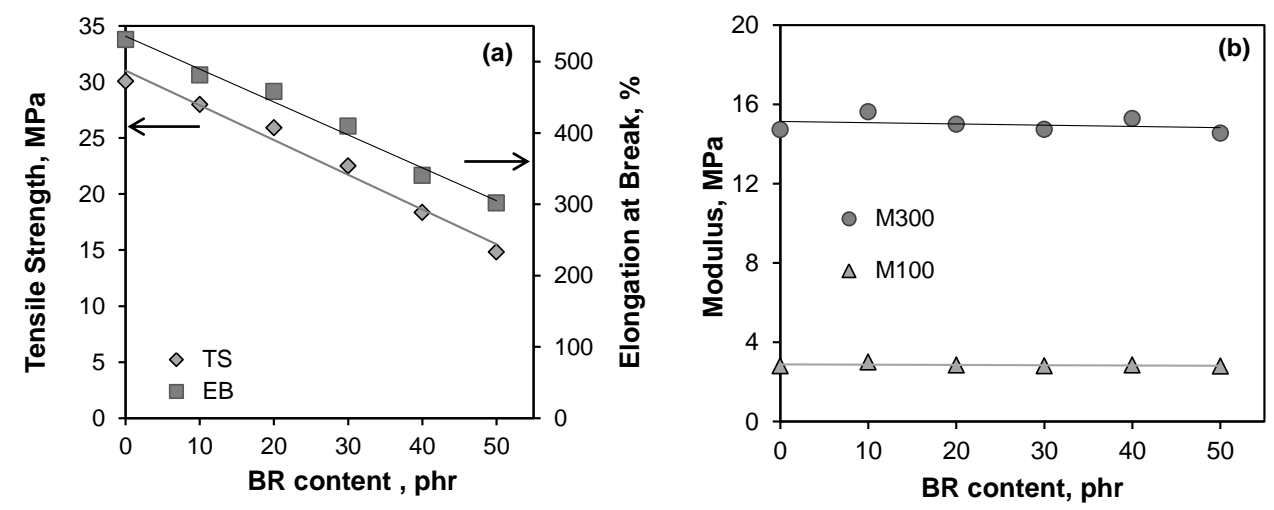

Figure 9.18 Tensile properties of NR/BR blends: (a): Tensile strength and elongation at break; (b): Modulus at 100 and $300 \%$.

\subsubsection{FTIR Characterization of NR/BR/IR Blend}

At $910 \mathrm{~cm}^{-1}$ is located the characteristic stretching vibration band of 1,2-vinyl in BR and IR as shown in Figure 9.19. The $910 \mathrm{~cm}^{-1}$ peak for the vinyl-group is strong for BR but for IR it is relatively weak.

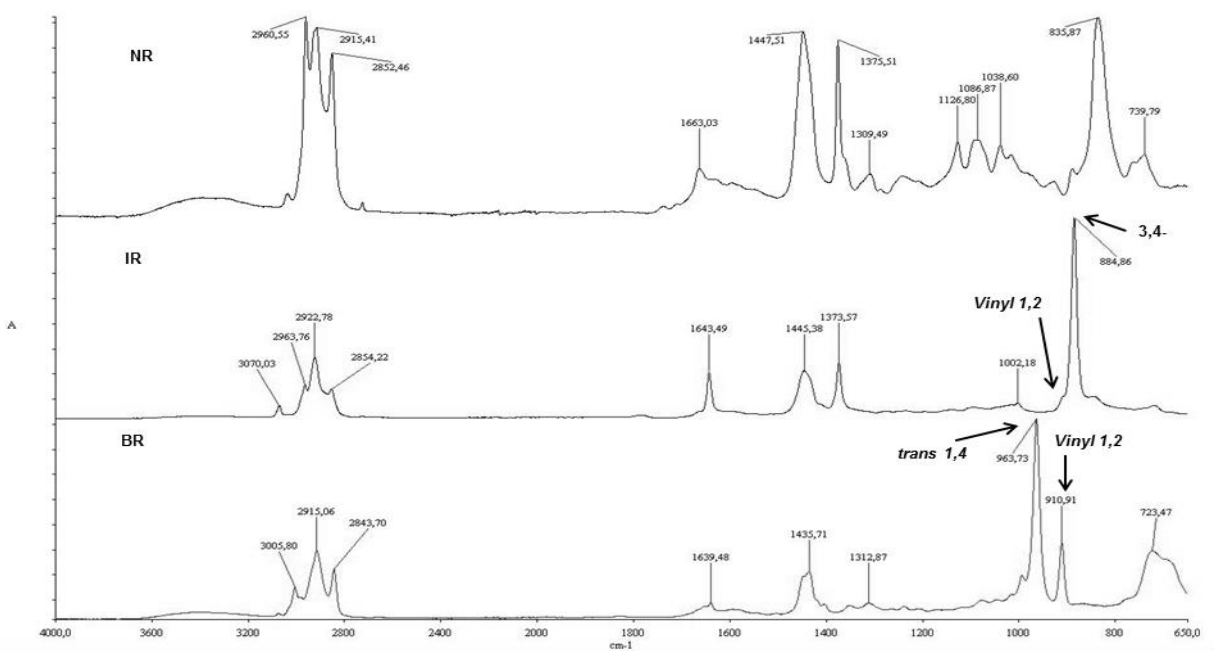

Figure 9.19 FT-IR spectra of raw NR, IR and BR showing the vinyl-1,2-peak at $910 \mathrm{~cm}^{-1}$.

The infrared spectra of a $80 / 10 / 10 \mathrm{NR} / \mathrm{BR} / \mathrm{IR}$ silica-filled blend as compared to pure NR, 90/10 NR/BR and 90/10 NR/IR blends are shown in Figure 9.20. The asymmetric Si-OSi peak located at $1075 \mathrm{~cm}^{-1}$ is a typical characteristic of silica compounds. The FTIR 
spectrum of 90/10 NR/BR blend only shows absorption peaks at $965 \mathrm{~cm}^{-1}$ for trans-1,4butadiene but no vinyl peak can be observed. Meanwhile, the 90/10 NR/IR blend is characterized by the 3,4-unit at $887 \mathrm{~cm}^{-1}$ and no vinyl peak is detected. The FTIR spectra of 80/10/10 NR/BR/IR blend also only shows the presence of bands at $965 \mathrm{~cm}^{-1}$ and $887 \mathrm{~cm}^{-1}$ for trans-1,4 and 3,4-units. The absence of the vinyl-peak in the blends is an indication that the vinyl-groups are completely reacted with silane. This indicates an improvement in the reactivity of silane towards rubber with incorporation of vinyl groups.

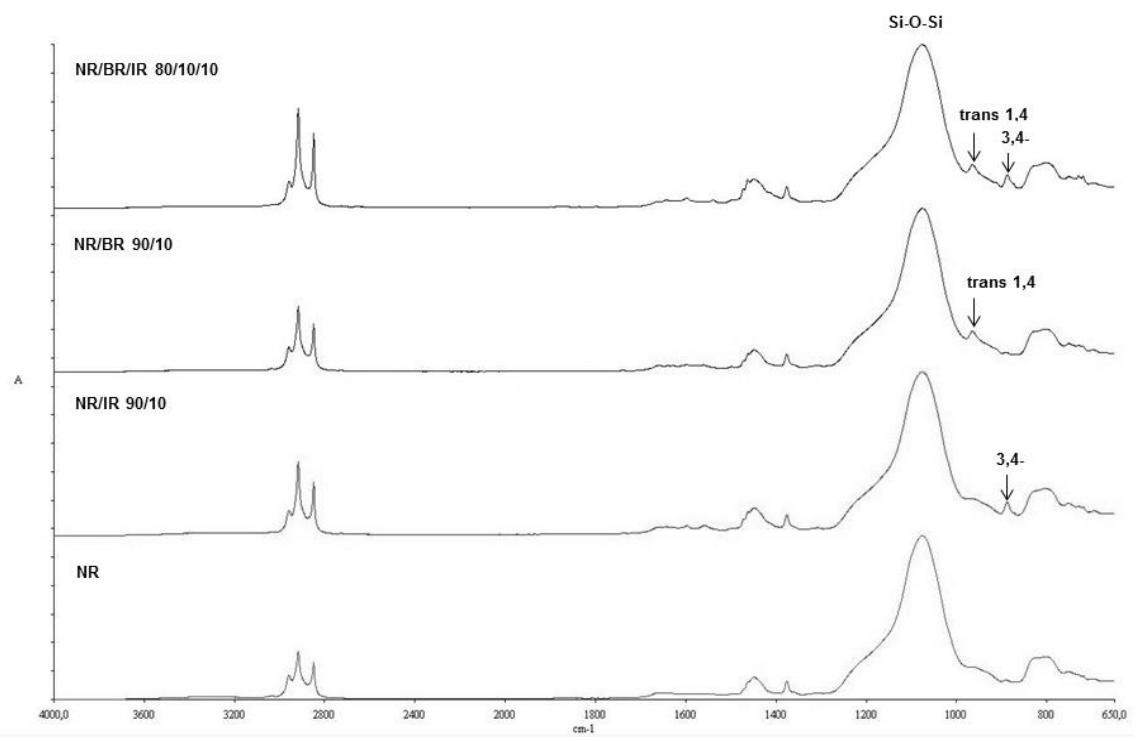

Figure 9.20 FT-IR spectra of a NR/BR/IR 80/10/10 blend with silica as compared to pure $N R$, as well as to NR/BR 90/10 and NR/IR 90/10 blends.

\subsubsection{Dynamic and Mechanical Properties of NR/BR/IR Blend}

The tan delta curve of NR/BR/IR $80 / 10 / 10$ blend as compared to $\mathrm{NR}$ as well as $90 / 10$ $\mathrm{NR} / \mathrm{BR}$ and NR/IR blends is shown in Figure 9.21. Based on DMA measurement at $10 \mathrm{~Hz}$, the $\mathrm{Tg}$ of the $\mathrm{NR} / \mathrm{BR} / \mathrm{IR}$ blend is $-43^{\circ} \mathrm{C}$ which is higher than those of $\mathrm{NR}\left(-48^{\circ} \mathrm{C}\right), 90 / 10$ $\mathrm{NR} / \mathrm{BR}\left(-48^{\circ} \mathrm{C}\right)$ and $90 / 10 \mathrm{NR} / \mathrm{IR}\left(-45^{\circ} \mathrm{C}\right)$ blends. It seems that the vinyl-IR has more influence than vinyl-BR on the dynamic properties of the NR/IR/BR blend. It relates to more compatibility of NR and IR as compared to NR and BR in the blend. The NR/BR/IR 80/10/10 blend does not show any improvement in the abrasion resistance and the low temperature flexibility. On the other hand, due to the higher $\tan \delta$ value at $0-30^{\circ} \mathrm{C}$, the $\mathrm{NR} / \mathrm{BR} / \mathrm{IR}$ blend has clear superiority for the wet skid resistance and braking performance. However, the 
$\mathrm{NR} / \mathrm{RR} / \mathrm{BR}$ blend also exhibits unfavorable rolling resistance and heat build-up properties as seen from the increase in the $\tan \delta$ value at temperature range $30^{\circ} \mathrm{C}$ to $70^{\circ} \mathrm{C}$. This is further shown in Figure 9.22(a) where the mechanical properties of the NR/IR/BR blend are also inferior to NR. Apart from that, the tan $\delta$ at $60^{\circ} \mathrm{C}$ of the NR/IR/BR blend is higher than for $\mathrm{NR}$, even though the reinforcing index M300/M100 is more or less comparable, as shown in Figure 9.22(b).

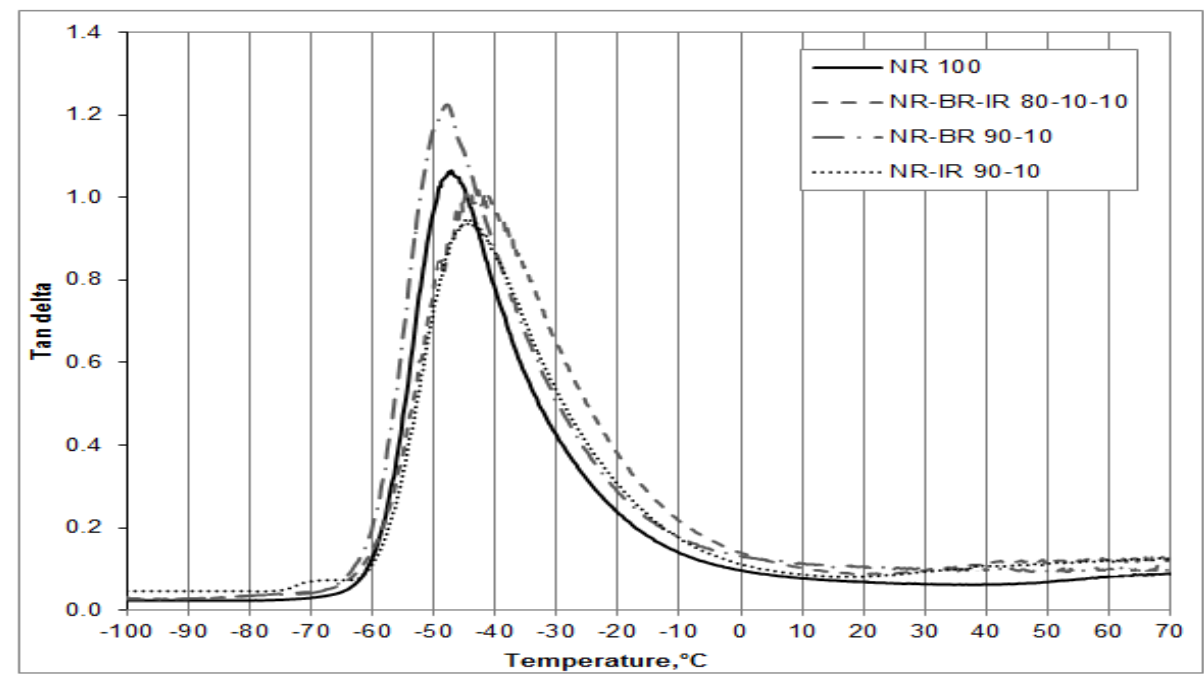

Figure 9.21 The tan delta curve of the NR/BR/IR blend (DMA 10Hz)
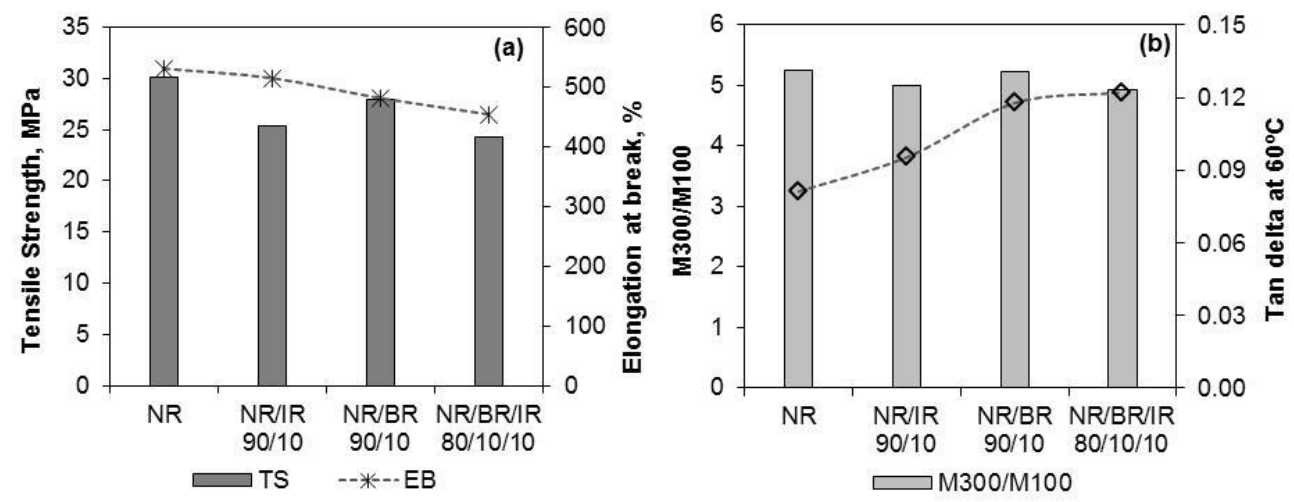

Figure 9.22 Comparison of (a)Tensile strength and Elongation at break, and (b) Reinforcing index M300/M100 and tan delta at 60ㄷ (DMA 10Hz) for NR/BR/IR 80/10/10 blend with NR and $N R / B R$ and $N R / I R$ blends. 
Comparison of the overall properties of the NR/BR and NR/IR blends related to tire requirements is shown in Figure 9.23. In all blends, improvement in the wet skid properties is obtained, however at the expense of higher rolling resistance. Expanding the magic triangle of tire technology is still quite a challenge!

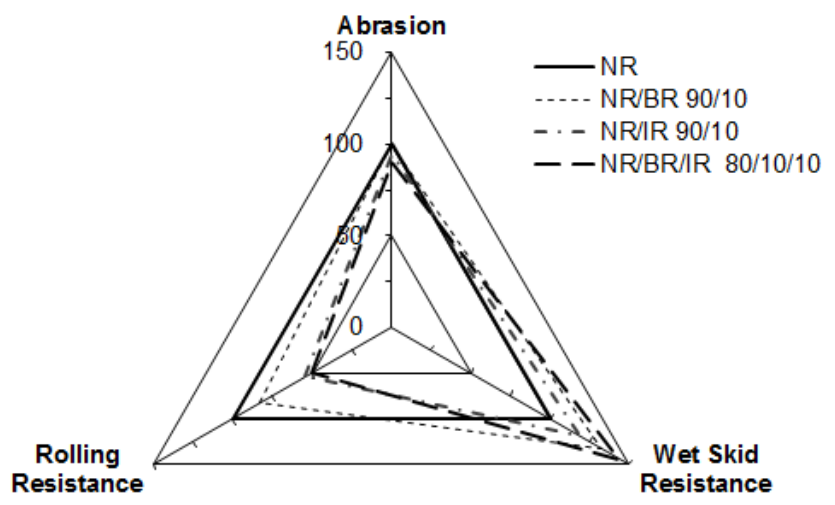

Figure 9.23 Overall properties of the blend compounds as compared to NR.

\subsection{CONCLUSIONS}

The phase angle, $\tan \delta$, is an important parameter for characterizing the viscoelastic behavior of elastomers and its relationship with dynamic tire properties. Elastomer macroand micro-structures, as well as the filler types are all major influencing factors to the overall shape of the hysteresis/temperature profile for a tread compound. The abrasion and skid resistance bears close relationship to the glass transition temperature, $\mathrm{Tg}$, of the elastomers. The choice of the rubber polymer with the optimum $\mathrm{Tg}$, or the proper combination of a blend of polymers, play a key role in achieving a compromise between the many tire requirements.

The blend of NR with $10 \%$-vinyl IR clearly improves the skid resistance as demonstrated by the higher tan delta in the range 0 to $30^{\circ} \mathrm{C}$. The $\mathrm{Tg}$ of the NR/IR blends shows a shift with the amount of $10 \%$-vinyl IR in the NR/IR blend, which translates into a reduction in abrasion resistance and low temperature flexibility of the NR/IR blends. The NR/IR blends give poor rolling resistance as indicated by the higher tan delta value at the range of 30 to $70^{\circ} \mathrm{C}$. Furthermore, the tensile properties are also negatively affected with the increase of $10 \%$-vinyl IR ratio in the NR/IR blend.

A decrease in $\mathrm{Tg}$ of the NR/BR blends is observed with increasing $10 \%$-vinyl BR ratio. The NR/BR blends exhibit superior abrasion resistance to NR, in addition to suitability 190 
for low temperature or winter performance. On the one hand, the wet skid resistance of the NR/BR blends is slightly better than of NR. However, a higher tan delta value is observed at the range of 30 to $70^{\circ} \mathrm{C}$, which gives a clear indication of poor rolling resistance for the NR/BR blends.

The $80 / 10 / 10$ of $\mathrm{NR} / \mathrm{BR} / \mathrm{IR}$ blend has a higher $\operatorname{Tg}\left(-43^{\circ} \mathrm{C}\right)$ than that of $\mathrm{NR}\left(-48^{\circ} \mathrm{C}\right)$. The NR/BR/IR blend has clear superiority for the wet skid resistance and braking performance as demonstrated by a higher tan delta value at the range between $0-30^{\circ} \mathrm{C}$. On the other hand, the $80 / 10 / 10$ of NR/BR/IR blend does not show improvement in the abrasion resistance and the low temperature flexibility. In addition, the NR/IR/BR blend also exhibits unfavorable rolling resistance and heat build-up properties.

The results demonstrate that incorporation of small amounts of vinyl-content via IR or $\mathrm{BR}, 1-5 \%$, provides a considerable change in dynamic properties of the blend compounds, especially in the wed skid properties. Optimization of the tire tread properties by polymer blending is still not sufficient to expand the magic triangle. The four elements in silica technology which are polymer, silica, coupling agent and mixing conditions need to be properly optimized to achieve a positive compromise between the tire properties.

\subsection{REFERENCES}

1. K.H. Nordsiek, Kautsch. Gummi Kunstst., 38, 178 (1985).

2. Sitoshi Mihara, Reactive Processing of Silica-Reinforced Tire Rubber: New Insight into the Time- and Temperature- Dependence of Silica Rubber Interaction, PhD thesis University of Twente, Enschede (2009).

3. J. D. Ferry, Viscoelastic Properties of Polymers," 3rd ed., John Wiley \& Sons, New York, Ch. 1 (1980).

4. D.E. Hall and J.C. Moreland, Am.Chem. Soc. Rubber Div. Meeting, Dallas, Texas, April 4-6 (2000).

5. I.R. Gelling, Vehicle Road Interaction, ASTM STP 1225, B.T. Kulakowski, Ed., American Society for Testing and Materials, Philadelphia, 107 (1994).

6. M.J. Wang, Rubber Chem. Technol., 71, 520 (1998).

7. M.J. Wang, Rubber Chem. Technol., 72, 430 (1999).

8. M.J. Wang, S. Wolff, and J-B. Donnet, Rubber Chem. Technol., 64, 559 (1991).

9. K.E. Polmanteer and C.W. Lentz, Rubber Chem. Technol., 48, 795 (1975).

10. S.Wolff, M.-J.Wang and E.-H.Tan, Rubber Chem. Technol., 66, 1359 (1992).

11. T.G. Fox, Bull. Am. Phys. Soc, 1, 123 (1956). 
Chapter 9 


\section{Appendix}

\section{A Study on Reactions of TESPT and Rubber in Model Compound Vulcanization}

The Model Compound Vulcanization technique is applied to investigate the mechanism of reactions of TESPT with rubber using liquid chromatography / mass spectroscopy with electrospray ionization (LCMS-ESI). 2,3-dimethyl-2-butene (TME) is used as a model rubber compound. A comparison is made between TESPT and TESPD in the model compound vulcanization. Interestingly, both the TESPT-TME and TESPD-TME reactions yield a crosslinked product similar to the product of model compound vulcanization of TME with the structure of TME-S $-T M E$, which indicates the role of sulfur donor of both silanes. 


\section{INTRODUCTION}

In enhancing the compatibility of a-polar rubbers and silica, bifunctional organosilanes such as bis(triethoxysilylpropyl)-tetrasulfide (TESPT) and bis(triethoxysilylpropyl)-disulfide (TESPD) are commonly used as coupling agents. The silane coupling agents improve the interaction on nano-scale by the creation of chemical links between the silica particles and the rubber molecules. The reaction mechanism between silica, TESPT silane coupling agent and rubber has been extensively reviewed ${ }^{1,2}$. On one side, the triethoxysilyl group of the TESPT reacts with the silanol groups of silica during compounding with generation of ethano $\left.\right|^{3-5}$. On the other side, the rubber reactive group of the silane (e.g. tetrasulfane) has a strong tendency to form rubber-to-filler bonds during curing of the rubber compounds $\mathbf{s}^{1,2}$.

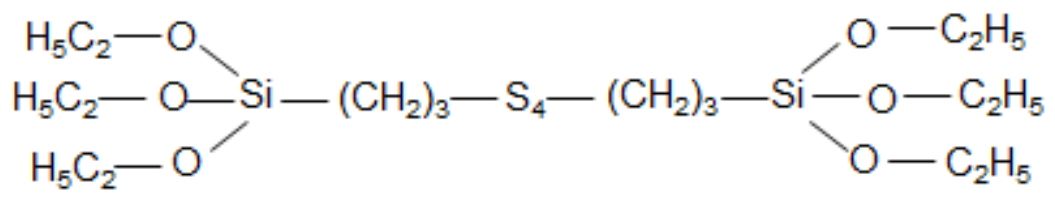

Figure A.1 Structural formula of bis(triethoxysily/propyl)-tetrasulfide (TESPT)

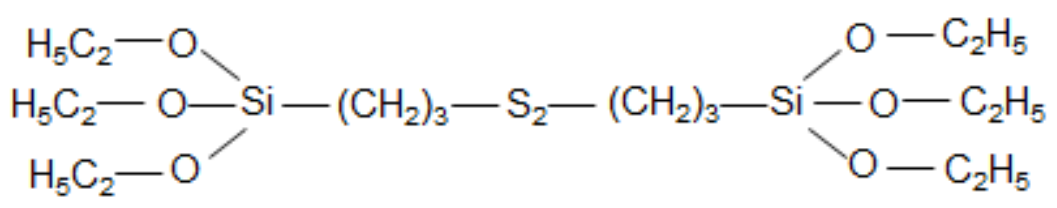

Figure A.2 Structural formula of bis(triethoxysilylpropyl)-disulfide (TESPD)

Model Compound Vulcanization (MCV) is a useful technique to investigate the vulcanization chemistry by using a low molecular weight model rubber compound ${ }^{6}$. Using MCV with squalene as model molecule, sulfur-accelerated vulcanization of natural rubber was characterized using HPLC, GPC and $\mathrm{MS}^{7-9}$. Görl et al. studied the influence of rubber chemicals on the reaction behavior of the TESPT sulfur moiety using a model rubber system ${ }^{10}$. Crosslinking reactions between TESPT and rubber take place as measured in a model olefin system even in absence of elemental sulfur, as demonstrated by Debnath et al. ${ }^{11}$ and Reuvekamp ${ }^{12}$. Liquid chromatography/ coordination ionspray-mass spectroscopy has been used for the identification of reaction products in model rubber compound vulcanization ${ }^{13}$. 
Liquid chromatography mass spectrometry (LC/MS) has evolved as one of the most important analytical tools for identification and analysis of reaction products. The techniques combines the LC-separation with highly selective mass detection. The interfaces for mass spectrometry are predominantly electrospray ionization (ESI) and atmospheric pressure chemical ionization (APCI), where both ionization techniques work under atmospheric pressure conditions. For ESI-MS, solvent and sample are nebulized in a high potential electrostatic field. The charged droplets are dried and ions are desorbed under the influence of the electrostatic field. The ions are then separated according to their mass-tocharge ratio $(\mathrm{m} / \mathrm{z})$ in an analyzer by electromagnetic fields.

The present chapter focusses on the comparison between the reactivity of TESPT and TESPD in model compound vulcanization. By applying the MCV technique, an understanding of the chemical reactions taking place between the sulfidic group in the organosilane and rubber during vulcanization is made possible. 2,3-dimethyl-2-butene (TME) is used as model rubber compound. TME has four equivalent allylic positions and due to its stability and simplicity, is a good choice to investigate the crosslinked products. The characterization of reaction products from the reaction of TME with TESPT and/or TESPD by the use of ESI-LCMS is described.

\section{EXPERIMENTAL}

\section{Materials}

The model rubber compound used for this study is 2,3-dimethyl-2-butene (TME), molecular

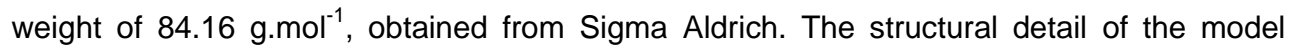
rubber compound is shown in Figure A.3. TME is generally used as model for all $\mathrm{a}-\mathrm{H}$ containing rubbers.

The silane coupling agents used are bis(triethoxysilylpropyl)-tetrasulfide (TESPT) and bis(triethoxysilylpropyl)-disulfide (TESPD) from Evonik, Germany. Solvents used for the HPLC mobile phase were acetonitrile (ACN) and water, both LCMS grades, supplied by Biosolve.

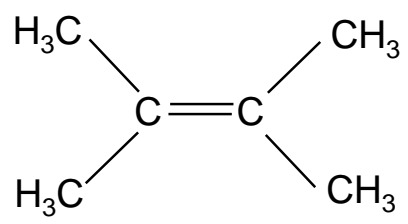

Figure A.3 Structural formula of 2,3-dimethyl-2-butene (TME) 


\section{Reaction procedure}

The reaction mixtures as listed in Table A.1 were weighed into special glass reaction ampoules as shown in Figure A.4. A magnetic stirrer was included in the ampoules. The ampoules were flushed with nitrogen gas and sealed by melting the neck.

The reaction was carried out by dipping the ampoules into an oil bath at $150^{\circ} \mathrm{C}$ for one hour. After the reaction, the ampoules were cooled immediately in an ice bath. The ampoules were opened and the reaction mixture was taken from the ampoule. About 100 $\mu \mathrm{L}$ of the reaction mixture was diluted with $5 \mathrm{~mL}$ acetonitrile. The diluted solution was filtered by using a $45 \mu \mathrm{m}$ Whatman porous filter. Of the filtered sample, $100 \mu \mathrm{L}$ were diluted with $900 \mu \mathrm{L}$ acetonitrile. The sample was analyzed using LC-MS(ESI interface).

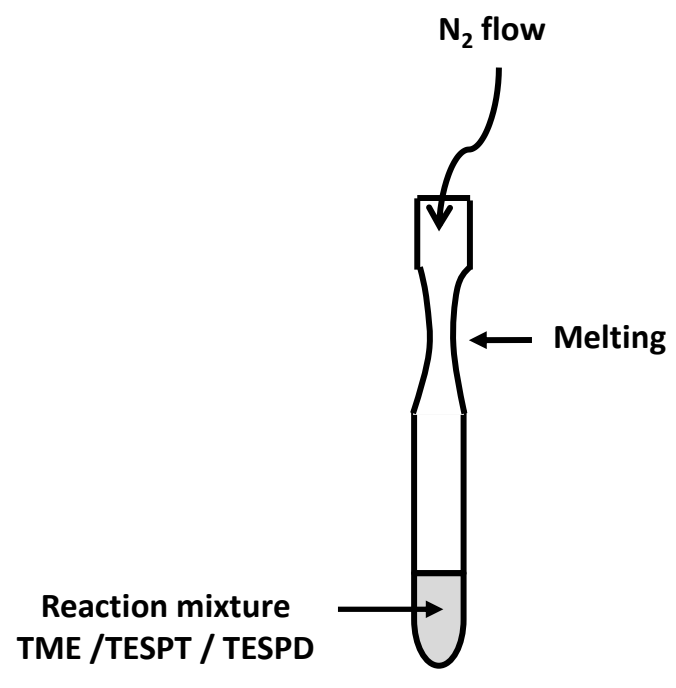

Figure A.4 Reaction mixture in reaction ampoule

Table A.1 Composition of reaction mixture ( in grams).

\begin{tabular}{llllll}
\hline & Mixture 1 & Mixture 2 & Mixture 3 & Mixture 4 & Mixture 5 \\
\hline TME & 1.00 & 1.00 & 1.00 & 1.00 & 1.00 \\
TESPT & - & 0.05 & 0.05 & - & - \\
TESPD & - & - & - & 0.05 & 0.05 \\
Sulfur & 0.014 & - & 0.014 & - & 0.014 \\
ZnSt & 0.025 & - & - & - & - \\
CBS & 0.017 & - & - & - & - \\
\hline
\end{tabular}




\section{HPLC/MS Analysis}

The HPLC/MS analysis was performed using an Agilent HPLC 1100 series, and Bruker MS Esquire 3000+. A reverse phase column (Nucleosil 100-5 C18 HD) with a length of $250 \mathrm{~mm}$ and an internal diameter of $4.6 \mathrm{~mm}$ was used for the separation. The mobile phase was a mixture of acetonitrile and water in 97:3 ratio. The flow rate of the mobile phase of 0.5 $\mathrm{ml} / \mathrm{min}$ was optimized for separation and MS ionization. The injected volume to the HPLC system was $10 \mu \mathrm{l}$. The wavelength detection by the UV detector was $254 \mathrm{~nm}$. After separation in HPLC, the reaction products were ionized using the Electrospray lonization (ESI) technique. Protonation in positive ion mode leads to adducts with sodium or potassium or ammonium. The MS analysis was carried out according to the conditions specified in Table A.2, and using Esquire 3000 software. The created ions were identified according to their $\mathrm{m} / \mathrm{z}$ ratio.

Table A.2 MS ionization condition in ESI

\begin{tabular}{ll}
\hline MS condition & \\
Flow rate & $0.5 \mathrm{~m} / / \mathrm{min}$ \\
Nebulizer gas pressure & $207 \mathrm{kPa}$ ( or $30 \mathrm{psi})$ \\
Dry gas flow & $3 / / \mathrm{min}$ \\
Dry gas temperature & $325^{\circ} \mathrm{C}$ \\
\hline
\end{tabular}

\section{Results AND Discussion}

\section{LC-MS Analysis of TESPT}

TESPT is actually a mixture of polysulfide silanes with different sulfur ranks, ranging from $\mathrm{S}_{1}$ to $S_{7}$; the average sulfur rank is about 3.83. The different components in TESPT can be separated by HPLC and identified based on the sulfur rank by mass spectrometry. A thorough analysis of TESPT is important in this study to use the retention times and reduce the complexity of the mass spectra of the reaction products later on. Figure A.5(a) shows the typical base peak chromatogram (BPC) from mass spectroscopy of TESPT showing the sulfur ranks from $S_{2}$ to $S_{7}$ at different retention times. It is observed that the $S_{3}$ peak has the highest intensity, followed by $S_{2}$ and then $S_{4}$. The mass spectra of TESPT-components with two to five sulfur atoms are presented in Figure A.5 (b) to (e), respectively. 

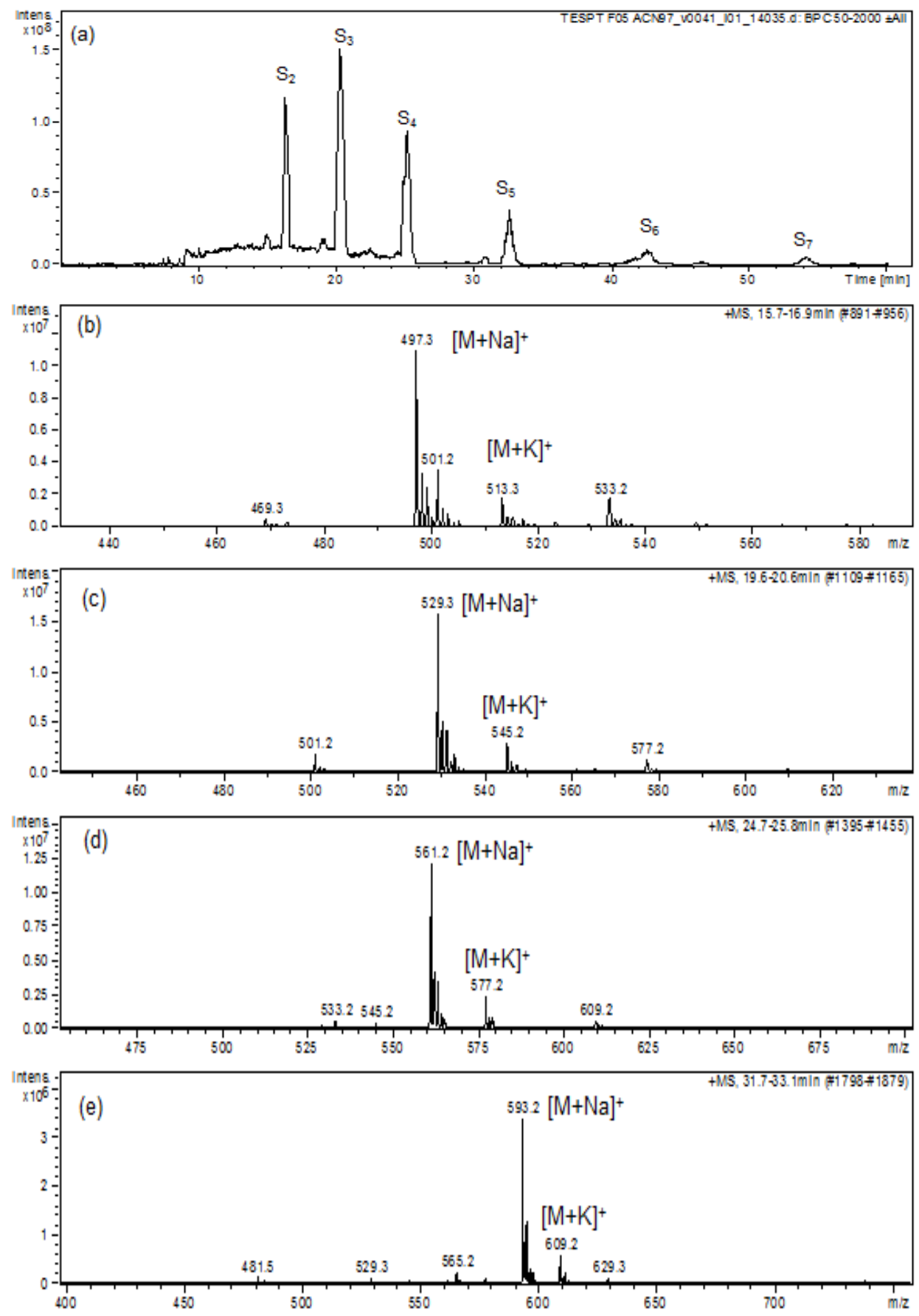

Figure A.5 (a) Base peak chromatogram of TESPT, and the corresponding mass spectra of (b) $S_{2}$-peak; (c) $S_{3}$-peak; (d) $S_{4}$-peak; (e) $S_{5}$-peak. 
In all cases, the formation of ion adducts with sodium $\left(\mathrm{Na}^{+}\right)$and potassium $\left(\mathrm{K}^{+}\right)$are observed. The mass spectrum of the $S_{2}$-peak as shown in Figure A.5(b) gives $\mathrm{m} / \mathrm{z}$ ratios of 497.3 and 513.3, which correspond to ion adducts in combination with $\mathrm{Na}^{+}(474.8+23)$ and $\mathrm{K}^{+}(474.8+39)$, respectively. The details of the ion adducts observed for all $\mathrm{S}_{2}-$ to $\mathrm{S}_{6}$-peaks of TESPT are represented in Table A.3.

Table A.3 lon adducts observed for TESPT according to sulfur rank

\begin{tabular}{lccc}
\hline Sulfur rank & MW & {$[\mathbf{M + N a}]^{+}$} & {$[\mathbf{M + K}]^{+}$} \\
\hline $\mathrm{S}_{2}$ & 474.2 & 497.3 & 513.3 \\
$\mathrm{~S}_{3}$ & 506.9 & 529.3 & 545.2 \\
$\mathrm{~S}_{4}$ & 538.9 & 561.2 & 577.2 \\
$\mathrm{~S}_{5}$ & 571.0 & 593.2 & 609.2 \\
$\mathrm{~S}_{6}$ & 603.1 & 625.2 & 641.0 \\
\hline
\end{tabular}

\section{Comparison of TESPT and TESPD by LC-MS Analysis}

A base peak chromatogram of TESPD as compared to TESPT is shown in Figure A.6. It is clearly noticed in the BPC of TESPD that the $S_{2}$-peak has the highest intensity relative to the other sulfur ranks. The $S_{3^{-}}$and $S_{4}$-peaks of TESPD are still visible at the same retention times as for TESPT, but with lower intensity than the $S_{2}$-peak. The extracted ion chromatograms (EIC) of $\mathrm{m} / \mathrm{z} 497, \mathrm{~m} / \mathrm{z} 529$ and $\mathrm{m} / \mathrm{z} 561$ from the BPC of TESPD are shown in Figure $A .7$ (a),(b) and (c) which correspond to the $S_{2^{-}}, S_{3^{-}}$and $S_{4}$-peaks, respectively. This confirms the existence of small amounts of polysulfides in TESPD as well.

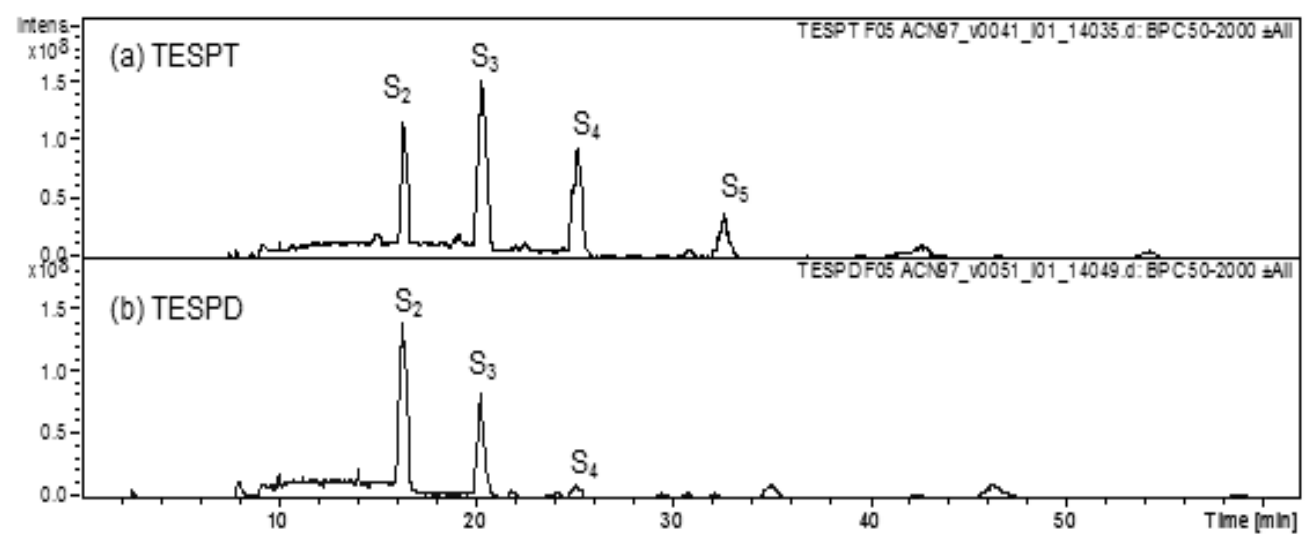

Figure A.6 Base peak chromatogram of (a) TESPT and (b) TESPD. 


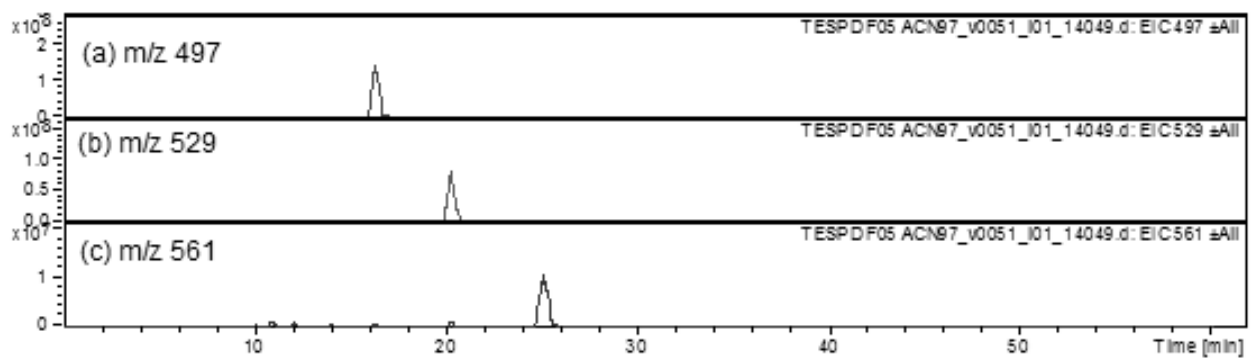

Figure A.7 Extracted ion chromatogram (a) (EIC) of $S_{2^{-}}$, (b) EIC of $S_{3^{-}}$and (c) EIC of $S_{4^{-}}$ peaks of TESPD.

\section{Reaction Mixture of TME Model Compound Vulcanization}

Figure A.8 shows the chromatogram by UV spectroscopy of the cross-linked products of TME model compound vulcanization (MCV) at a reaction temperature of $140^{\circ} \mathrm{C}$ for one hour, in the presence of sulfur, zinc stearate and CBS: Mixture 1. The crosslinked products of TME MCV with difference in sulfur rank can be identified in the UV chromatogram. This agrees well with the work of Debnath ${ }^{11}$ and Rajan ${ }^{14}$. The structures of the crosslinked products are shown in Figure A.9, where they consist of two TME molecules bridged by several sulfur atoms (TME-S - TME).

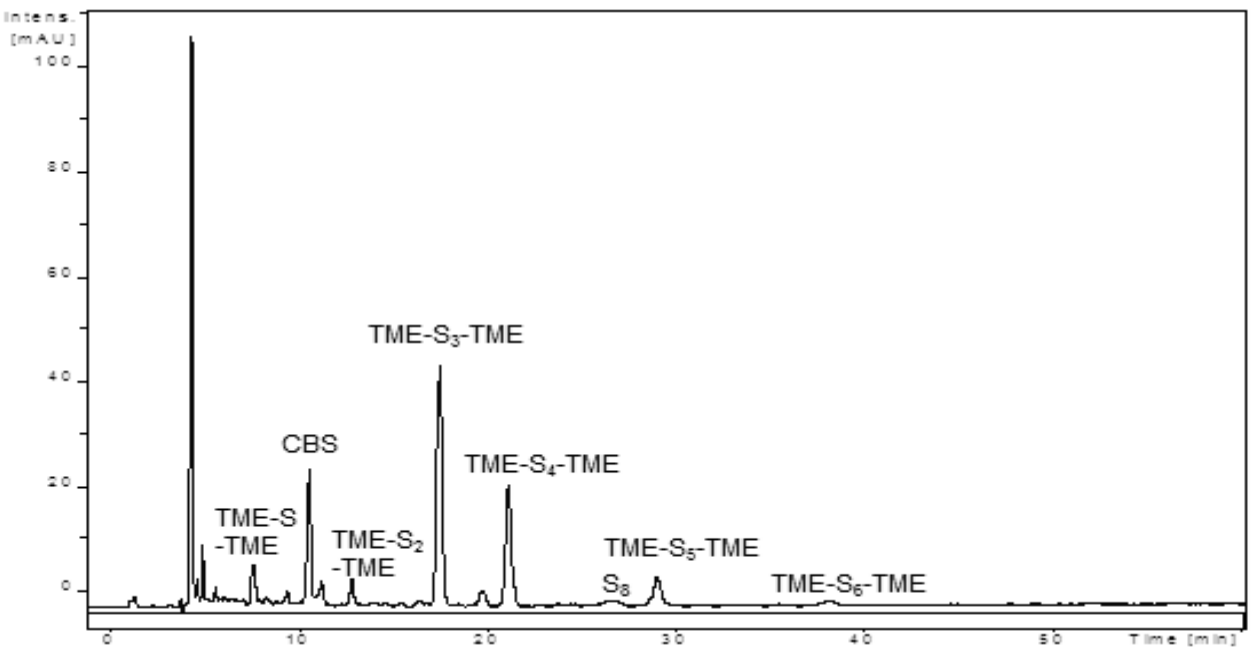

Figure A.8 HPLC chromatogram of the reaction products from TME model compound vulcanization (Mixture 1). 
The BPC of reaction mixture 1 of TME model compound vulcanization is shown in Figure A.10. The peak of CBS is confirmed by the MS at 10.3 minutes retention time as shown in Figure A.11. The mass spectra of the crosslinked products, TME-S $3-T M E, T M E-$ $\mathrm{S}_{4}-\mathrm{TME}$ and TME-S $-\mathrm{TME}$ are given in the Figure A.12. The crosslinked products, TME- $\mathrm{S}_{\mathrm{X}^{-}}$ TME are difficult to be ionized. In addition, the concentration of TME-S 5 -TME is very low, and hence the peak is very small. The same is observed for the mono- (TME-S-TME) and di-sulfidic crosslinked TME (TME-S ${ }_{2}-\mathrm{TME}$ ), where no spectrum can be observed from MS as the concentration is too low and unfavorable for normal ionization. Other methods such as addition of silver salts $\left(\mathrm{AgBF}_{4}\right)$ or silver $\left(\mathrm{Ag}^{+}\right)$ions is considered as a promising alternative to enhance the ionization ${ }^{13}$, however this was not carried out in this work as it required a different experimental set-up.<smiles>CC(C)=C(C)CS[As]CC(C)=C(C)C</smiles>

Figure A.9 Structure of the crosslinked products, TME-S $-T M E$, and $x$ corresponds to number of sulfur atoms.

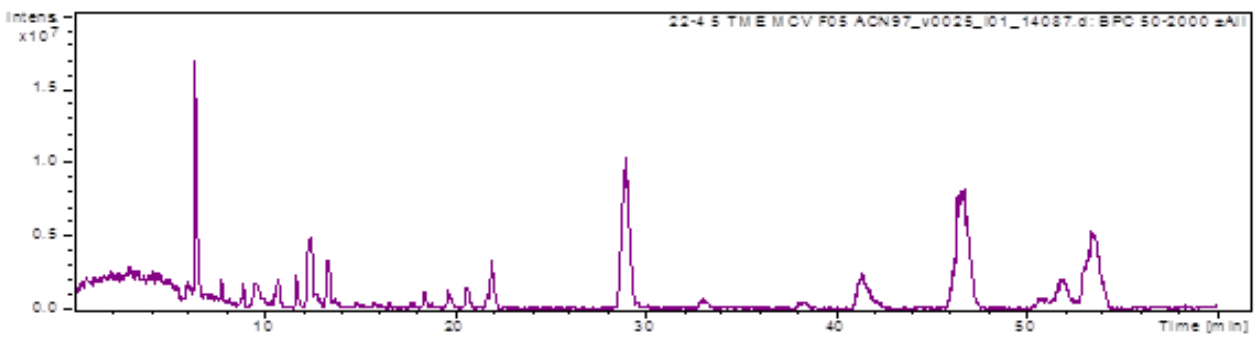

Figure A.10 Base peak chromatogram of reaction products from TME model compound vulcanization (Mixture 1).

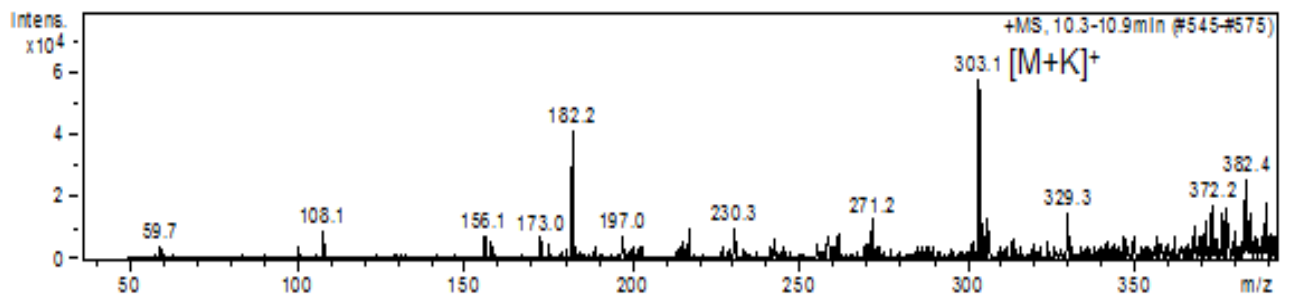

Figure A.11 Mass spectrum of CBS, $303 \mathrm{~m} / \mathrm{z}$ at 10.3 minutes retention time 

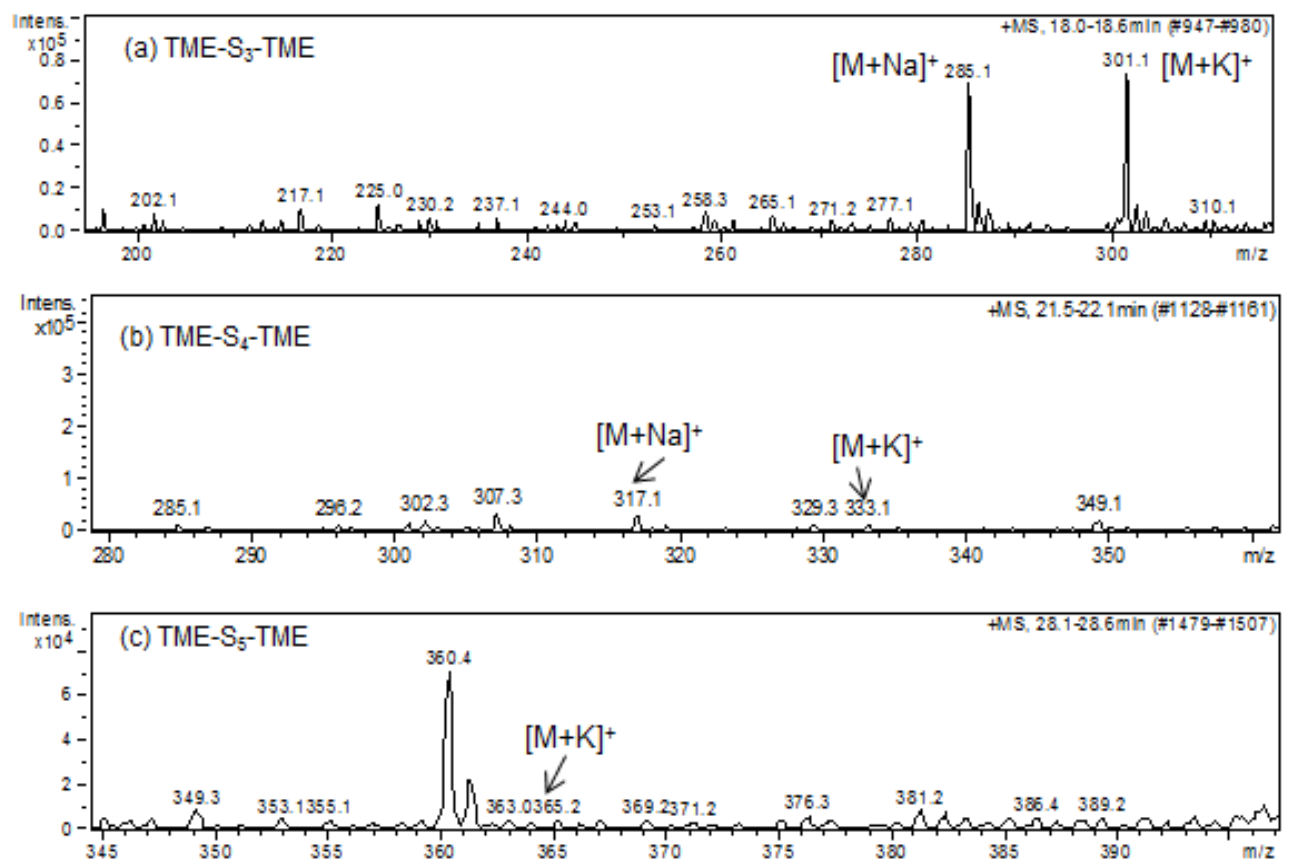

Figure A.12 Mass spectrum of crosslinked products of TME: (a) TME-S ${ }_{3}-T M E$;

(b) TME-S $4-T M E$ and (c) TME-S $5-T M E$.

\section{Reaction mixture of TME and TESPT}

Comparison of the chromatogram by UV trace between TME MCV (Mixture 1), TESPT and reaction products of TME and TESPT (Mixture 2) is shown in Figure A.13. The UV chromatogram of reaction products of TME and TESPT has both the peaks from TESPT and TME MCV. Hence, the retention time from the TESPT and TME MCV can be used to identify the reaction products of TME and TESPT as shown in Figure A.14. It can be seen that the same crosslinked product of TME is obtained from the reaction of TME with TESPT without any accelerator. The crosslinked product, TME-S $-T M E$ is formed from sulfur released from TESPT during the reaction. It demonstrates the role of TESPT as sulfur donor, even in the absence of any cure accelerators

In Figure A.15, the BPC from MS spectroscopy of the reaction mixture of TME with TESPT as compared to the BPC of TESPT is shown. It is clearly visible that the $\mathrm{S}_{2^{-}}, \mathrm{S}_{3^{-}}$and $\mathrm{S}_{4}$-peaks of TESPT are still visible in the TME-TESPT reaction mixture 2. The BPC for the TME-TESPT reaction mixture shows that the $\mathrm{S}_{4}$-peak has a lower intensity than the $\mathrm{S}_{3}$ - and $\mathrm{S}_{2}$ - peaks and at the same time the $\mathrm{S}_{2}$-peak has the highest intensity. It also indicates a decrease in the concentrations of polysulfides of TESPT as the reaction progresses. 

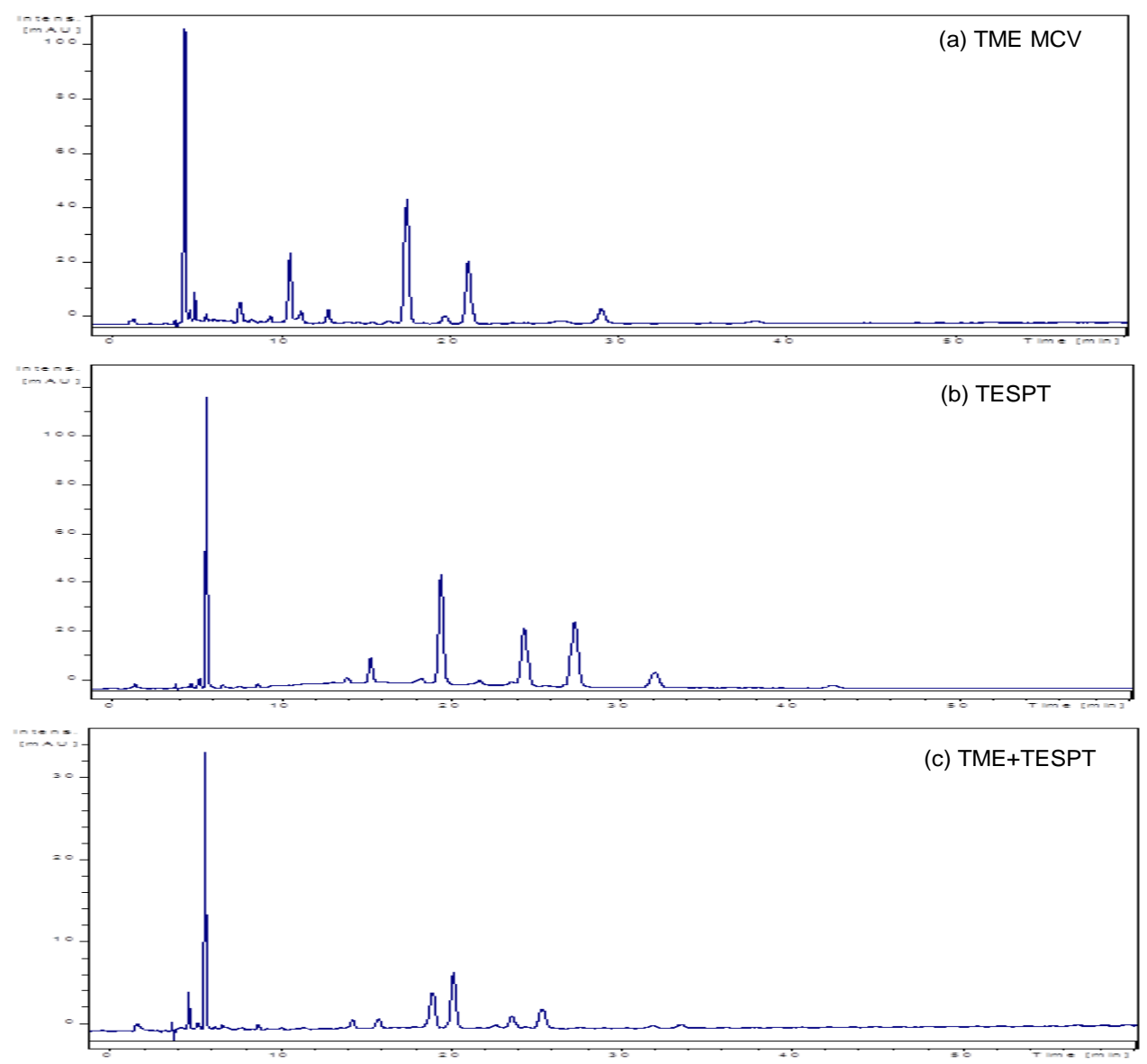

Figure A.13 Comparison of HPLC chromatogram of (a) model compound vulcanization of TME; (b) TESPT; and (c) reaction of TME and TESPT.

An additional peak at 22-23 minutes of retention time is also observed from Figure A.15 .The mass spectra of the corresponding peak at 22-23 minutes is shown in Figure A.16, where it is identified as the crosslink reaction product of TME. The $\mathrm{m} / \mathrm{z} 413.4$ is identified as a positively charged ion of TME croslinked product in combination with sodium $(M 390+23)$. Meanwhile, the $\mathrm{m} / \mathrm{z} 803.6$ belong to the same TME crosslinked product adduct ion with $\mathrm{Na}^{+}$but with double mass $(2 \mathrm{M}+23)$, which is typical for electronspray ionization. The crosslinked product structure is shown in Figure A.17 where it consists of two TME molecules bridged by four sulfur atoms (TME-S - TME- $\left.\mathrm{S}_{2}-\mathrm{SH}\right)$. 


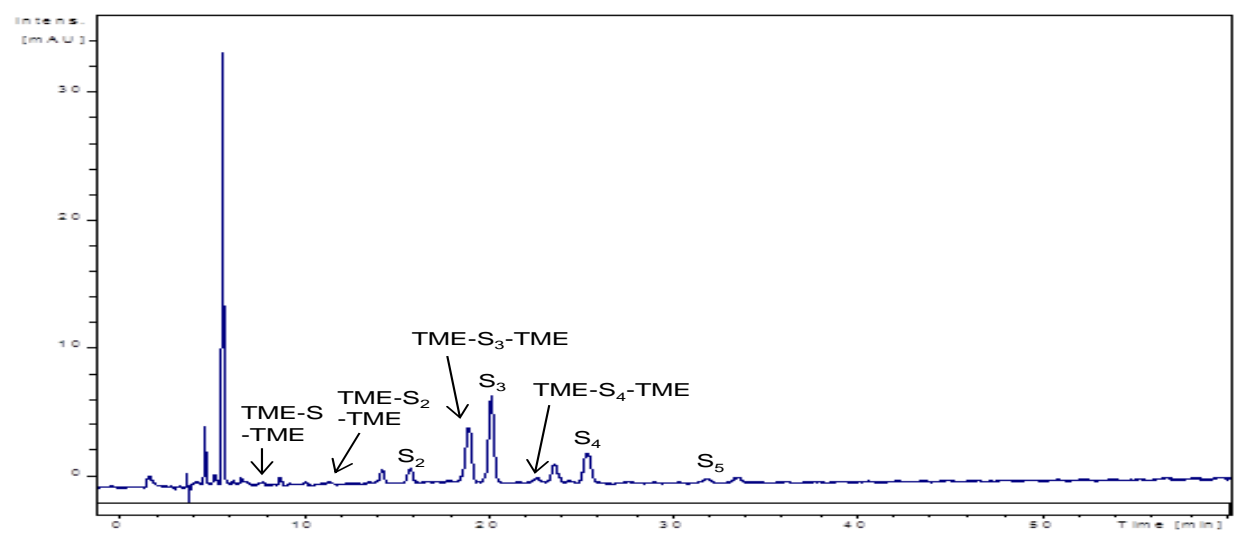

Figure A.14 The UV chromatogram of the reaction products from the reaction of TME and TESPT (Mixture 2).

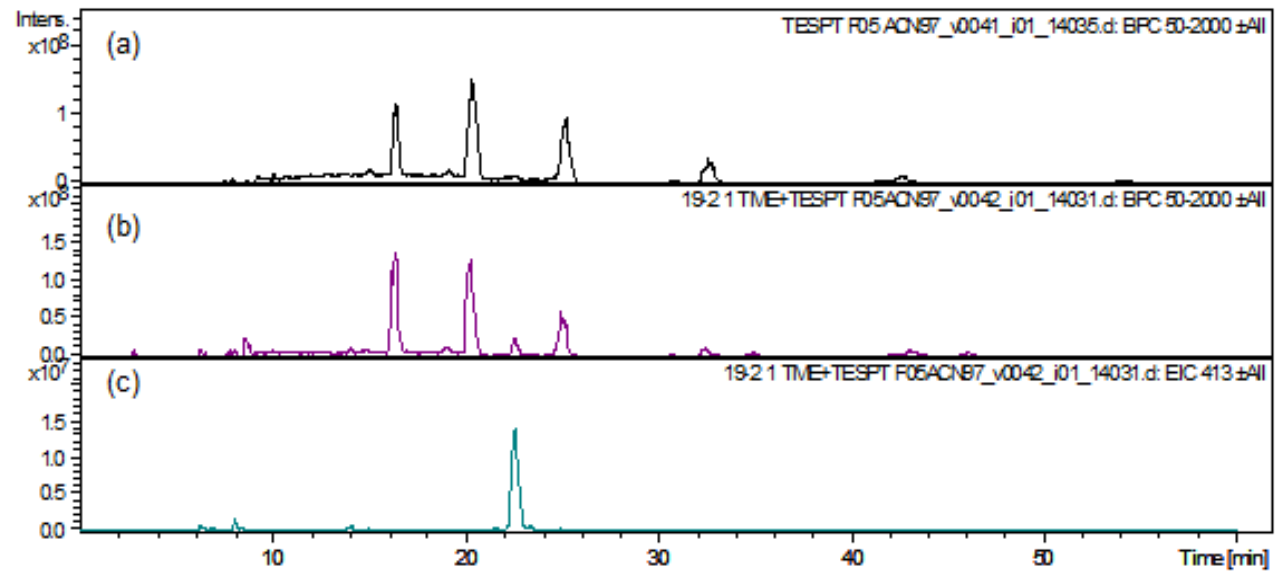

Figure A.15 Base peak chromatogram of (a) TESPT, (b) TME and TESPT reaction product, and (c) extracted ion chromatogram of $\mathrm{m} / \mathrm{z} 413$.

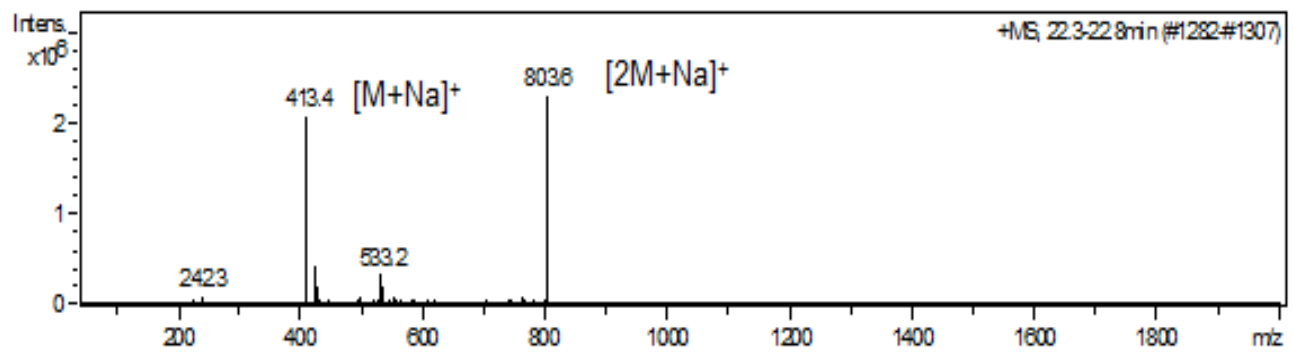

Figure A.16 Mass spectrum of TME /TESPT reaction product at 22-23 minutes. 


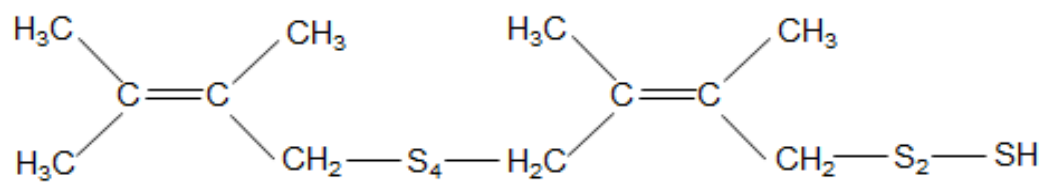

Figure A.17 Structure of the crosslinked product corresponding to $\mathrm{m} / \mathrm{z} 413.4$.

The mechanism of the formation of crosslinked product, TME-Sx-TME from reaction of TESPT and TME is shown schematically in Scheme A.1. Upon heating at sufficiently high temperature, TESPT will disproportionate into bis(triethoxysilylpropyl)disulfide and bis(triethoxysilylpropyl)-polysulfides. During this disproportionation, sulfur radicals are released from TESPT, which involve in the vulcanization reaction. The active sulfur radicals then react with an allylic carbon in TME to form a TME-S ${ }_{x}$ radical. The TME$S_{x}$ radical subsequently reacts with another TME molecules to form crosslink products, TME-S ${ }_{x}$-TME. In addition, the TME radicals also can undergo recombination to produce crosslinked TME.

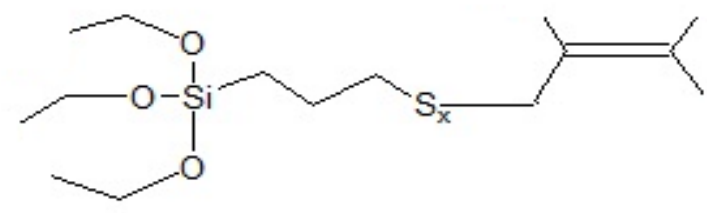

Figure A.18 A TESPT-TME product, triethoxy silylpropyl 2,3 dimethyl-2-butene polysulfides

The results of our TME and TESPT reaction product are identified as polysulfidic crosslinked TME (Figure A.9). In that perspective, it is anticipated that the recombination of the TME-S $S_{x}$ radicals is most probable. In addition, TESPT-TME products would be expected from the triethoxysilylpropylthiol radical with TME molecules, which are triethoxysilylpropyl2,3-dimethyl-2-butene polysulfides, as shown in Figure A.18. However, no TESPT-TME product could be detected from the mass spectra. This is also in agreement with findings of Debnath $^{11}$ and Reuvekamp ${ }^{12}$. One of the probable reasons is that the triethoxysilylpropylthiol radicals prefer to recombine to form bis(triethoxysilylpropyl)-disulfide and -trisulfide during the disproportionation of TESPT. This would also explain the high and 
stable $S_{2-}$ and $S_{3}$-peaks in the mass spectra of TME and TESPT reaction (Figure A.15). However, the formation of bis(triethoxysilylpropyl)-polysulfides is limited as the sulfur radicals are already used for crosslinking of TME. Another possibility is the thermal decomposition of the triethoxy-silylpropyl-2,3-dimethyl-2-butene polysulfides (TESPT-TME) product as described by Debnath et al. ${ }^{11}$.

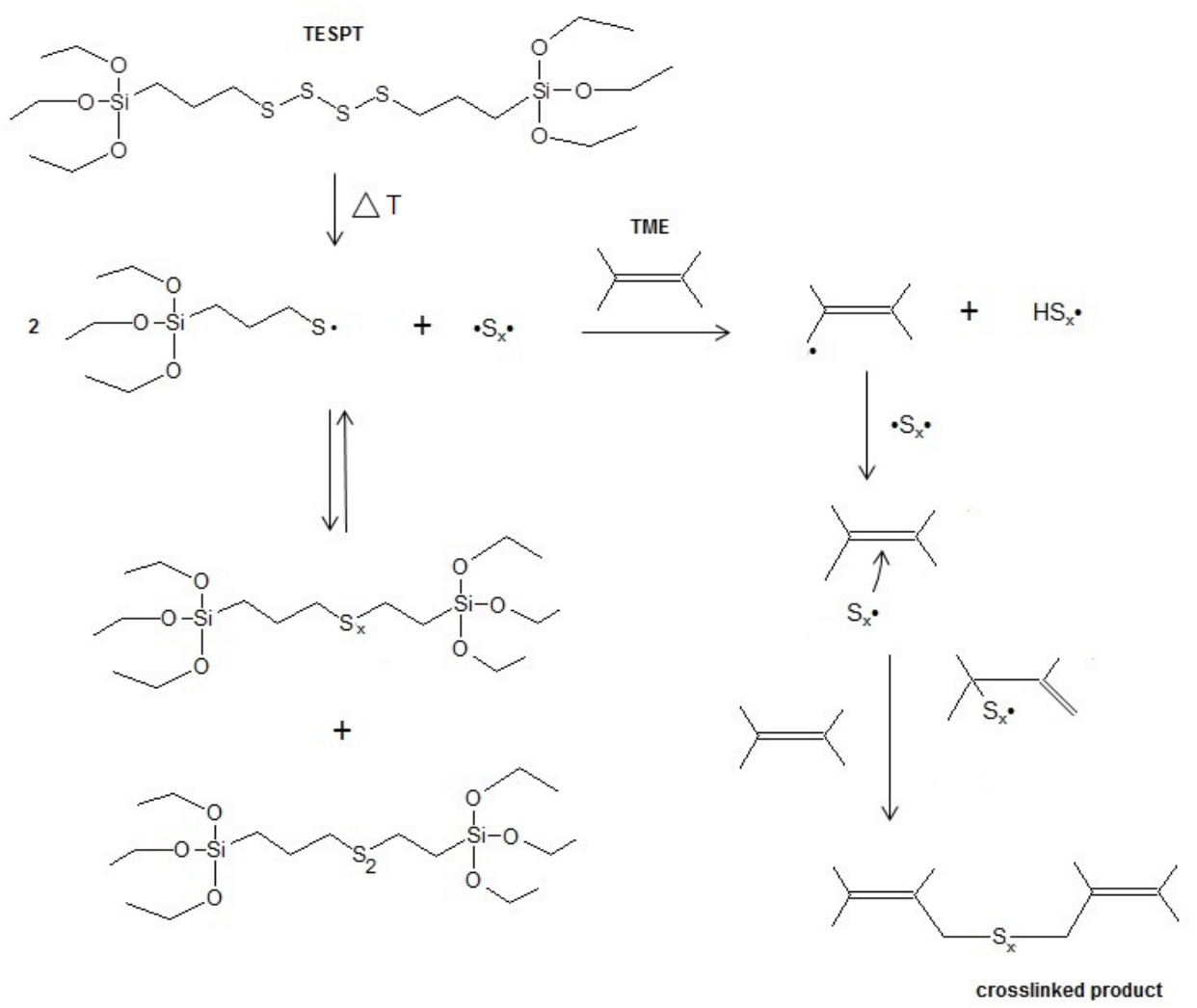

Scheme A.1 A schematic mechanism showing the release of sulfur during the disproportionation of TESPT which leads to the formation of crosslinked products of TME.

\section{Reaction mixture of TME, TESPT and Sulfur}

The chromatogram by UV spectroscopy of the reaction product of reaction mixture 3: TME, TESPT and sulfur is shown in Figure A.19. The crosslinked products, TME-S - TME with $x$ ranging from 1 to 5 sulfur atoms, are formed from reaction mixture 3 . In addition, the sulfur peak is also visible. In the presence of sulfur in the reaction of TME and TESPT, more polysulfidic crosslinked products with increased concentration are obtained. 
Figure A.20 shows the BPC of the reaction of TME, TESPT and sulfur. The $\mathrm{S}_{2^{-}}$, $S_{3^{-}}, S_{4^{-}}, S_{5^{-}}$, and $S_{6}$-peaks of TESPT are still visible as also observed from the UV trace. In the presence of sulfur in the reaction mixture 3, TESPT also accepts sulfur and increases the concentration of the higher sulfur ranks. However, the crosslinked products are not detected by the mass spectrum, which may be due to the difficulty to ionize the vulcanizates.

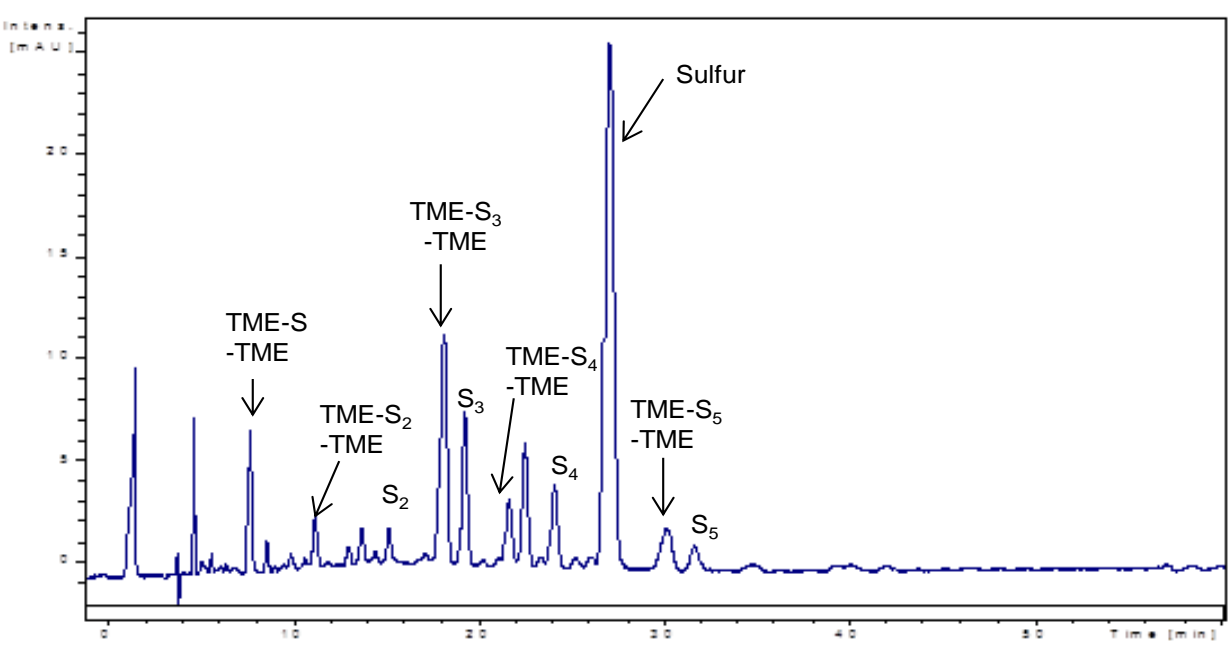

Figure A.19 The UV chromatogram of the reaction mixture 3 of TME, TESPT and sulfur.

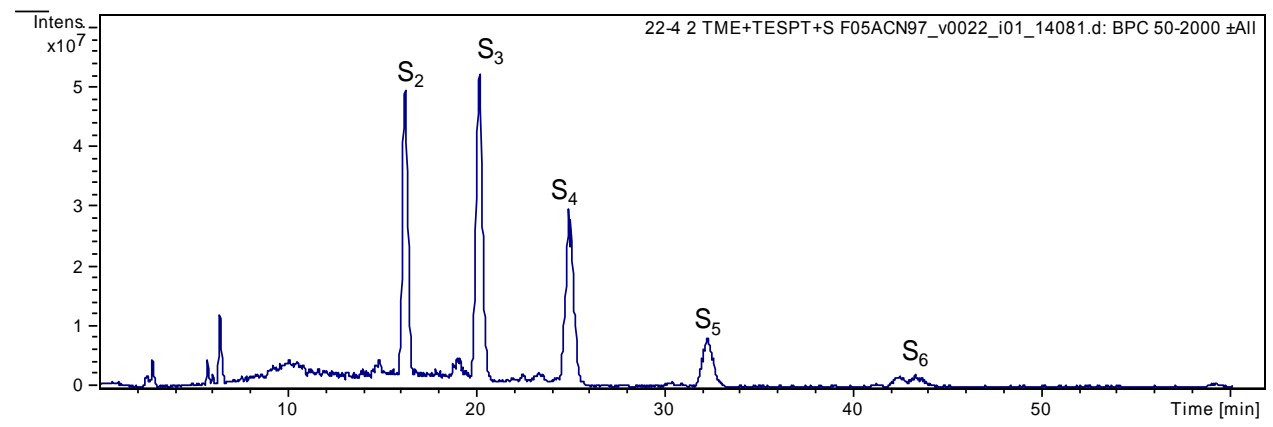

Figure A.20 Base peak chromatogram of reaction mixture 3: TME, TESPT and sulfur.

\section{Reaction mixture of TME and TESPD}

The chromatogram by UV spectroscopy of the reaction product of TME and TESPD, Mixture 4, is shown in Figure A.21. Only one reaction product can be detected which is monosulfide crosslinked TME. In addition, the $\mathrm{S}_{2}$ - and $\mathrm{S}_{3}$-peaks from TESPD are also visible. 
The BPC of the reaction mixture 4 of TME with TESPD as compared to TESPD is shown in Figure A.22. The $S_{2-}$ and $S_{3}$-peaks of TESPD are still observed in the TMETESPD reaction mixture, as also observed from the UV trace. The reaction of TME with TESPD leads to a crosslinked product of TME bridged by a sulfur atom, TME-S-TME.

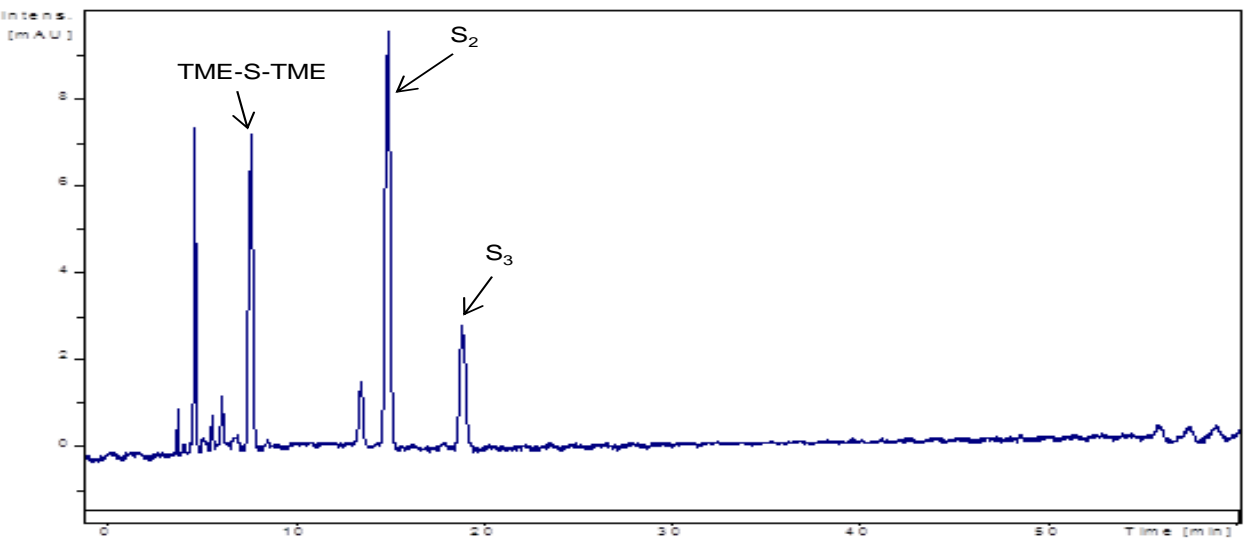

Figure A.21 The UV chromatogram of the reaction mixture 4 of TME and TESPD.

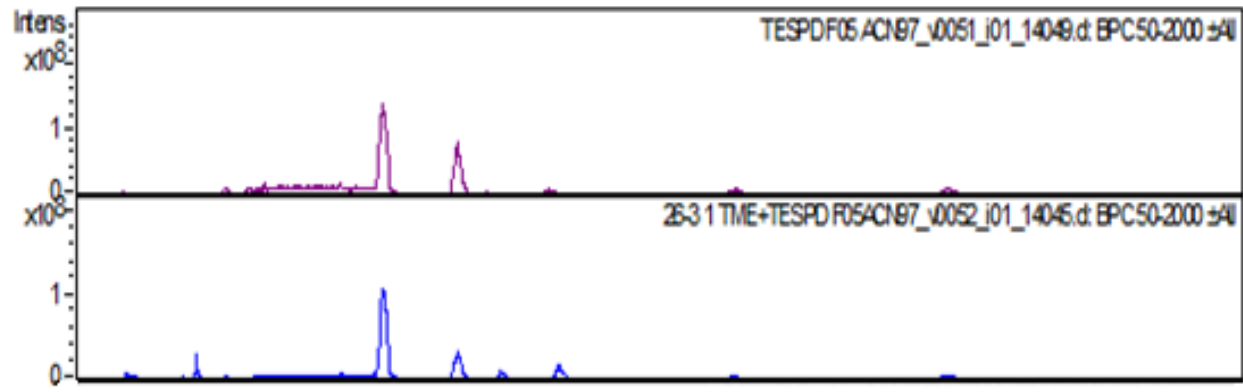

Figure A.22 Base peak chromatogram of (a) TESPD, (b) TME and TESPD reaction product.

However, the reaction product is not detected by the mass spectrum. The formation of TME-S-TME during the reaction of TME and TESPD is again the result of the sulfur donor effect of TESPD. However, the reactivity of TESPD towards TME is less compared to TESPT. The mechanism of crosslinking of TME as discussed in scheme A.1 also applies for the TME-TESPD reaction. Hence, the results show that both TESPT and TESPD have the role as sulfur donors which contribute to the crosslinking of the rubber compound. 


\section{Reaction mixture of TME, TESPD and Sulfur}

The chromatogram by UV spectroscopy of the reaction product of reaction mixture 5: TME, TESPD and sulfur is shown in Figure A.23. The peaks of crosslinked products, TME-S $\mathrm{X}^{-}$ TME, ranging from 1 to 4 sulfur atoms are observed as well as the peak for sulfur. The BPC of the reaction of TME, TESPD and sulfur is shown in Figure A.24. Only $S_{2^{-}}, S_{3^{-}}$and $S_{4^{-}}$ peaks of TESPD can be observed and the crosslinked products are not detected by the mass spectrum.

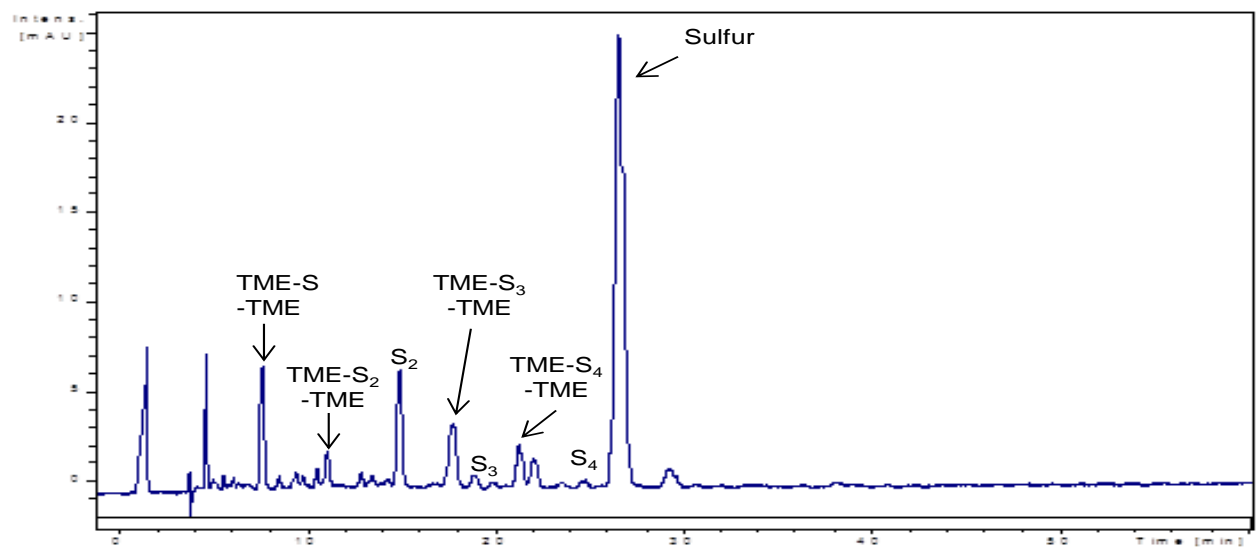

Figure A.23 The UV chromatogram of the reaction mixture 5 of TME, TESPD and sulfur.

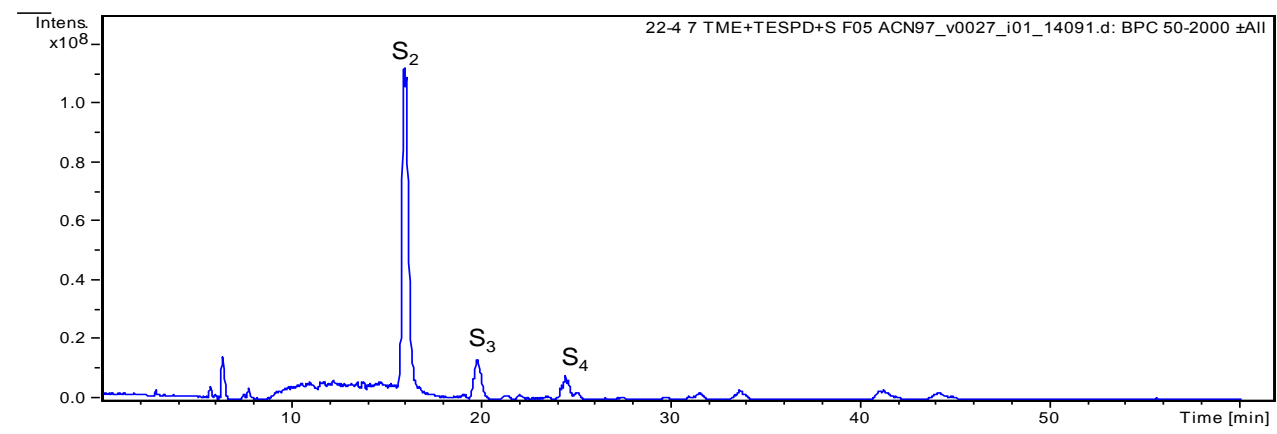

Figure A.24 Base peak chromatogram of reaction mixture 5: TME, TESPD and sulfur.

\section{CONCLUSIONS}

The role of silane coupling agent, TESPT as a sulfur donor in the vulcanization of rubber is illustrated in this model compound vulcanization study. The model compound vulcanization of TME in the presence of vulcanization agents yields crosslinked products with a structure 
of TME-S $-T M E$, where $x$ represents the number of sulfur atoms ranging from 1 to 6 . The reaction of TESPT with the TME model rubber compound produces a crosslinked product of polysulfidic linkage, even in the absence of any vulcanization accelerators. Interestingly, in this study both TESPT-TME and TESPD-TME reaction products have a similar structure of the crosslinked products as MCV of TME, as observed from HPLC. However, identification of the structure with MS-ESI did not succeed, mainly due to the difficulties to ionize the compounds. The mechanism of the formation of crosslinked products from the sulfur released by TESPT is presented.

\section{REFERENCES}

1. W. Meon, A. Blume, H.-D, Luginsland and S. Uhrlandt, "Silica and Silane". In Rubber Compounding Chemistry and Applications, ed.: B. Rodgers, Marcel Dekker Inc., New York, USA. (2004).

2. J.W.M. Noordermeer and W.K. Dierkes, "Silica-Filled Rubber Compounds", in Rubber Technologist's Handbook, Vol.2, eds.: J. White, S.K. De and K. Naskar, Smithers Rapra Technology, Shawbury, Shrewsbury, Shropshire, UK. (2008).

3. U. Görl, A. Hunsche, A. Müller and H.G. Koban, Rubber Chem. Technol., 70, 608 (1997).

4. A. Hunsche, U. Görl, A. Müller, M. Knaack and Th. Göbel, Kautsch. Gummi Kunstst., 50, 881 (1997).

5. A. Hunsche , U. Görl, H.G. Koban, and Th. Lehmann, Hürth, Kautsch. Gummi Kunstst., 51, 525 (1998).

6. P.J. Nieuwenhuizen, J.G. Haasnoot and J. Reedijk, Kautsch. Gummi Kunstst., 53, 144-148. (2000).

7. S. Rodriguez, C. Massales, N. Agulló, S. Borrós, L. Comellas and F. Broto, Kautsch. Gummi Kunstst., 52, 438 (1999).

8. S. Borrós and N. Agulló, Kautsch. Gummi Kunstst., 53, 131-136 (2000)

9. E. Vidal-Escales and S. Borros, Talanta, 62, 539 (2004)

10. U. Görl, J. Münzenberg, D. Luginsland and A. Müller, Kautsch. Gummi Kunstst., 52, 588 (1999).

11. S.C. Debnath, R.N. Datta and J.W.M. Noordermeer, Rubber Chem. Technol., 76, 1311 (2003).

12. L.A.E.M. Reuvekamp, PhD thesis, University of Twente, Enschede, the Netherlands (2003).

13. H. Hayen, M.M. Alvarez-Grima, S.C. Debnath, J.W.M. Noordermeer and U. Karst, Anal. Chem., 76, 1063 (2004).

14. V.V. Rajan, W.K. Dierkes, J.W.M. Noordermeer and R. Joseph, Rubber Chem. Technol., 80, 40 (2007). 


\section{Summary and Outlook}

\section{SUMMARY}

The choice of the four essential elements in silica-technology: the rubber polymer, the type of silica, an effective coupling agent and the appropriate mixing technology, are important in order to expand the magic triangle of tire technology: the compromise between rolling resistance, (wet) traction and wear. The present trend of raw material usage towards eco-friendly elastomers is an impetus for research into and increased usage of Natural Rubber (NR). As extensively reviewed in Chapter 2, silica has a positive influence on reduced rolling resistance and lower heat build-up of tires as compared to carbon black in NR compounds. The critical factor in silica technology is the nano-scale coupling between rubber molecules and the silica surface via silane coupling agents, not to be disturbed by other surface-active agents like proteins. However, little information on the effect of nonrubber constituents, particularly proteins on the silica-filled NR compounds is known. Hence, in the first part of this thesis, the role of non-rubber constituents, particularly proteins on silica-silica and silica-silane interactions is investigated, to understand the mechanism of silica reinforcement in NR compounds.

The key element in the reinforcement of rubber by silica is the chemical coupling between silica and rubber, which applies for both synthetic and natural rubbers. The investigation in Chapter 3 demonstrates that the temperature development is an important parameter in mixing silica and natural and synthetic polyisoprene (IR) with the aid of TESPT as coupling agent in order to achieve proper silanization of silica. Silica-silica interaction is reduced with hydrophobation of the silica surface by TESPT through silanization at sufficiently high mixing temperatures. In NR compounds, the silanization reaction between silica and TESPT progresses with increasing mixing dump temperature. Above an optimum dump temperature of approximately $150^{\circ} \mathrm{C}$, NR suffers in loss of dynamic and mechanical properties probably due to thermo-mechanical degradation. This effect is even more pronounced in the case of IR. On the other hand, removal of proteins from NR, in this case Deproteinized Natural Rubber (DPNR), results in a smaller influence of dump temperature and more constant mechanical properties. Dynamic properties improve with the silica-silanerubber coupling as evidenced by a lower $\tan \delta$ at $60^{\circ} \mathrm{C}$ obtained especially in DPNR-silicaTESPT compounds. 
Coupling agent and proteins show a complicated antagonistic effect in silica reinforcement of natural rubber as demonstrated in Chapter 4. The effect of proteins is most pronounced when no silane is used in NR-silica compounds. When high amounts of proteins are present in NR, such as in skim rubber, a low Payne effect is observed which is comparable to silane-containing compounds. This demonstrates that the interactions between proteins and silica are able to disrupt the silica-silica networking. For NR and low protein content rubber DPNR, silanization of silica is increased at sufficiently high mixing temperatures. However, mixing temperature has little influence on the properties of a high protein-content skim rubber compound. This indicates that the hydrophobation of the silica surface by silane may be partially hindered due to silica-protein interactions.

The influence of proteins on silica-filled NR is further detailed in Chapter 5. TEM Network Visualization of vulcanizates without silane reveals vacuoles around the silica particles and aggregates, which indicate weak filler-to-rubber interactions. This is further supported by only physically bound rubber in the compounds without silane. For NR and DPNR vulcanizates without silane, strong filler-filler interactions as observed from the higher Payne effect contribute to their physically bound rubber. In contrast, skim rubber vulcanizates exhibit lower Payne effects due to disruption of the silica-silica network by proteins. Even though proteins are capable of hydrophobizing the silica surface and reducing the filler-filler interaction, they do not contribute to strong rubber-to-filler interactions, as the silica-protein interaction only leads to formation of physically bound rubber. In comparison, the vacuoles are more clearly observed in DPNR and skim rubbervulcanizates without silane than in the NR-vulcanizate. The rubber-to-filler interaction of silica-filled natural rubber vulcanizates is greatly improved with the use of silane TESPT as coupling agent. The TEM Network Visualization of the silica-vulcanizates with TESPT shows no formation of vacuoles, which demonstrates strong attachment of the rubber network to silica aggregates. This is the result of a chemical reaction between silica and TESPT, as also demonstrated by a high chemically bound rubber content and a low Payne effect. The results from the TEM Network Visualization and shift in the glass transition temperature confirm a strong rubber-to-filler interaction in the silica compounds with silane, and a minimal influence of protein in the presence of silane.

Filler-filler interactions in silica compounds relate to the dispersion morphology. In Chapter 6, a better insight into filler-filler interaction is obtained from the micro- and nanodispersion of silica by AFM and TEM measurements. The results show that the use of coupling agent TESPT enhances the micro-dispersion of silica, which results in an increase of filler-to-rubber interactions. In the presence of TESPT silane coupling agent, primary 
particles of silica of $50 \mathrm{~nm}$ and silica aggregates in the size of $100 \mathrm{~nm}$ are clearly visible in NR and DPNR vulcanizates. A bound rubber layer is also observed on the primary particles of silica in the DPNR-TESPT vulcanizate. In addition, the silica aggregates in DPNR are slightly more homogeneously dispersed than those in NR, indicating a good microdispersion of silica in DPNR-TESPT, which correlates well with the lower Payne effect of the vulcanizate. Silica networking is clearly very strong in skim rubber, both in the absence and presence of a silane coupling agent. The interaction between silica and proteins in skim rubber vulcanizates is further illustrated by the TEM nano-dispersion, where large protein globules of size approximately $300 \mathrm{~nm}$ block a good nano-dispersion of the silica aggregates; possibly also by preferred adsorption of the coupling agent TESPT.

The mechanism of silica-proteins interaction as compared to silica-silane interaction in hydrophobation of silica surfaces is illustrated in Chapter 7. The kinetics of the silanization of silica is faster in IR than in NR, as demonstrated by the lower activation energy of the former. An in-rubber study into the effect of NR serum proteins shows that silica-protein interactions lead to reduced filler-filler interaction and suppress the flocculation of silica. However, when both TESPT and proteins are used in combination in a silica compound, they compete for the silica surface. The competitive effect of protein and TESPT to hydrophobize the silica surface is reflected by a higher Payne effect. The mechanism of proteins in hydrophobation of silica surfaces is further demonstrated using a flat glass surface and compared to silica powder. The contact angle measurement shows a strong hydrophobation effect of NR serum protein on the silica surface, where the value is close to $90^{\circ}$.

The second part of this thesis deals with different alternatives to address the challenges for improving silica reinforcement in NR. In Chapter 8, the use of pre-treated or surface-modified silica to reinforce NR is demonstrated. The use of surface-modified silica eliminates the need for a coupling agent during mixing. The commercial surface-modified precipitated silica, Agilon ${ }^{\mathrm{TM}} 400$ is a mercapto-organometallic and non-coupling agent treated silica. The modified-silica is easily incorporated in NR during mixing as the silica surface is already silanized and has higher hydrophobicity which enhances its compatibility with NR. The use of modified-silica improves the macro-dispersion of silica in NR and prevents silica flocculation especially at high silica loadings. Increasing the modified-silica loading in both NR and DPNR compounds increases the maximum torque of the vulcanization curve, Mooney viscosity and Payne effect. By using ATR-FTIR spectroscopy, the amount of modified-silica in the compound can be monitored by the location of the peak absorbance in the silica region between $1250-1000 \mathrm{~cm}^{-1}$. The peaks of silica absorbance in 
both NR and DPNR vulcanizates are shown to shift to a lower wavenumber with increasing silica modified-silica loading. For an increase of modified-silica of $10 \mathrm{phr}$, the wavenumber of the peak of the silica region is decreased by $3.5 \mathrm{~cm}^{-1}$. The results show that there is no major difference in mechanical properties between NR and DPNR vulcanizates filled with modified-silica. The tensile strength, elongation at break and reinforcement index M300/M100 ratio, decrease with increasing silica loading. The increase in tan delta at $60^{\circ} \mathrm{C}$ and hysteresis energy loss for both NR and DPNR vulcanizates is proportional to the amount of the modified-silica used. In comparison with in-situ TESPT treated silica, the modified-silica shows a significant improvement in tan delta at $60^{\circ} \mathrm{C}$ for $\mathrm{NR}$, although for DPNR no difference is seen.

The choice of the elastomer with an optimum $\mathrm{Tg}$, or the proper combination of a blend of elastomers, play a key role in achieving a compromise between the many tire requirements. Chapter 9 focuses on the reinforcement of a silica-silane system in NR using blends of NR/IR and NR/BR. Both IR and BR are selected to have $10 \%$ vinyl-content. $A$ blend of NR with BR is a step to improve the abrasion resistance of a NR-based tread compound. In addition, blending of NR with vinyl-IR is to improve the reactivity of silicasilane-rubber coupling. The $\mathrm{Tg}$ of the NR/IR blends shows a shift with the amount of $10 \%-$ vinyl IR in the NR/IR blend, which translates into a reduction in abrasion resistance and low temperature flexibility of the NR/IR blends. The NR/IR blends clearly improve the skid resistance but give poor rolling resistance as demonstrated by the higher tan delta in the range 0 to $30^{\circ} \mathrm{C}$ and the higher tan delta at the range of 30 to $70^{\circ} \mathrm{C}$, respectively. $\mathrm{A}$ decrease in $\mathrm{Tg}$ of the NR/BR blends is observed with increasing $10 \%$-vinyl BR ratio. The NR/BR blends exhibit superior abrasion resistance to NR. On the one hand, the wet skid resistance of the NR/BR blends is slightly better than for NR. However, a higher tan delta value is observed at the range of 30 to $70^{\circ} \mathrm{C}$, which gives an indication of poorer rolling resistance for the NR/BR blends.

The $80 / 10 / 10$ of NR/BR/IR blend has a higher $\operatorname{Tg}\left(-43^{\circ} \mathrm{C}\right)$ than that of NR $\left(-48^{\circ} \mathrm{C}\right)$. The NR/BR/IR blend has clear superiority for the wet skid resistance and braking performance as demonstrated by a higher tan delta value at the range between $0-30^{\circ} \mathrm{C}$. On the other hand, the 80/10/10 NR/BR/IR blend does not show improvement in the abrasion resistance and the low temperature flexibility. In addition, the NR/IR/BR blend also exhibits unfavorable rolling resistance and heat build-up properties. The results demonstrate that incorporation of small amounts of vinyl-content via IR or BR, 1-5\%, provides a considerable change in dynamic properties of the blend compounds, especially in the wet skid properties. Optimization of the tire tread properties by polymer blending is still not sufficient to expand 
the magic triangle. The four elements in silica technology which are polymer, silica, coupling agent and mixing conditions need to be properly optimized to achieve a positive compromise between the tire properties.

In addition to the silanization reaction, which has been investigated in the previous chapters, pre-crosslinking reactions can take place during mixing NR-silica-TESPT compounds due to sulfur released from the TESPT. Hence, in the Appendix, the role of TESPT as a sulfur donor in the vulcanization of rubber is illustrated in a limited model compound vulcanization study. The reaction of TESPT and Tetra methyl ethylene (TME) produces crosslinked products with a structure of TME-S $-\mathrm{TME}$, even in the absence of any vulcanization accelerators. Interestingly, both TESPT-TME and TESPD-TME reaction products have a similar structure of the crosslinked products of polysulfidic linkage. The mechanism of the formation of crosslinked products from the sulfur released by TESPT is presented.

Overall, the reinforcement of silica in natural rubber is quite a challenge. In silica technology, the controlled silanization reaction between silica and silane is critical. However, NR is intrinsically less thermally stable than synthetic elastomers, while rather high temperatures and long durations are needed for a coupling agent to react primarily with silica and later with rubber polymers. In addition, NR is a natural product, subject to seasonal variations. The influence of non-rubber constituents on silica reinforcement in NR has been explored in this thesis. The effect of proteins is most pronounced with a high amount and when no silane is used in the compound. There is competitive effect of silane and proteins, when used at equal amounts, in hydrophobation of the silica surface. On the other hand, the use of DPNR for a silica-silane system gives improvement in the dynamic and mechanical properties, as well as the tan delta at $60^{\circ} \mathrm{C}$ of the vulcanizates. Nonetheless, the combination of silica technology with NR will produce more environmentally-friendly tires, and open up for numerous new and exciting researches on silica technology for NR. 


\section{SUGGESTIONS FOR FURTHER RESEARCH}

The present thesis provides insight into many aspects of NR for use in highdispersion silica tire tread compounds. The results of this thesis are expected to be a guide and starting point for further studies on silica technology for NR, as well as for NR itself.

Different types of natural rubbers have been investigated in this thesis. What is interesting is the good properties of DPNR, where it gives more consistent physical properties as compared to regular NR. A fundamental study on the network structure of DPNR which contribute to its excellent properties is of interest. In addition, there is a potential use of DPNR for silica-silane systems for tire-treads. Hence, optimization of parameters for silica-TESPT-DPNR compounds in terms of formulation and mixing conditions should be elaborated.

The work described in this thesis has been concentrating on TESPT as the coupling agent. An investigation on different types of coupling agents on silica reinforced NR would be a topic of further study. Various coupling agents from sulfur-containing silanes, such as mercaptosilanes and blocked silanes, as well as sulfur free silanes, can be optimized for suitability with NR, and more in depth studies have to be performed.

The strong hydrophobation effect of proteins on the silica surface is very fascinating. With the advantage of protein engineering, it would be interesting to use protein such as NR serum protein as compatibilizing agent for reinforcement of NR with silicacontaining fillers in compounds for non-tire applications. 


\section{Samenvatting}

\section{SAMENVATtING}

De volgende vier elementen zijn essentiëel voor silica-technologie in rubbertoepassingen: het rubber polymeer, het type silica, een effectief koppelingsmiddel (coupling agent) en een geschikte mengtechnologie. Deze elementen zijn belangrijk om de magische driehoek van de autobanden technologie te vergroten: het compromis tussen rolweerstand, grip op nat wegdek en slijtage. De huidige trend om het gebruik van grondstoffen in de richting van milieuvriendelijke elastomeren te verplaatsten, heeft geleid tot een toename in het gebruik van Natuur Rubber (NR) en was een aanzet voor dit onderzoek. Zoals uitgebreid beschreven in Hoofdstuk 2, heeft silica een positieve invloed op een lagere rolweerstand en een verminderde warmteontwikkeling van banden in vergelijking met roet in op NR gebaseerde rubbermengsels (compounds). De kritische factor in de silica-technologie is, dat de koppeling tussen de rubber moleculen en het silica oppervlak, via silaan coupling agents, niet wordt verstoord door andere oppervlakte-actieve stoffen, zoals eiwitten. Er is echter weinig informatie over het effect van de niet-rubber bestanddelen, vooral eiwitten op de silica-gevulde NR compounds bekend. Vandaar dat in het eerste deel van dit proefschrift de rol van niet-rubber bestanddelen, vooral eiwitten op silica-silica en silica-stilaan interacties is onderzocht om het mechanisme van silica-versterking in NR compounds te begrijpen.

Het belangrijkste element in de versterking van rubber met silica is de chemische koppeling tussen silica en rubber, hetgeen geldt voor zowel synthetische en natuurlijke rubbers. Uit het onderzoek in Hoofdstuk 3 blijkt, dat het temperatuurverloop tijdens het mengen van silica in natuurlijke en synthetische polyisopreen (IR) met behulp van TESPT als coupling agent een belangrijke parameter is om een juiste silanisatie van silica te bereiken. Silica-silica interactie wordt met hydrofobering van het silica oppervlak verminderd met TESPT, door silanisering bij voldoende hoge meng-temperaturen. In NR compounds is silanisering de reactie tussen silica en TESPT, welke wordt bevorderd naarmate de lossings-temperatuur tijdens het mengen hoger wordt. Boven een optimale lossingstemperatuur van ongeveer $150{ }^{\circ} \mathrm{C}$ treedt er voor NR verlies op van de dynamische en mechanische eigenschappen, waarschijnlijk door thermo-mechanische degradatie. Dit effect is nog meer uitgesproken in het geval van IR. Aan de andere kant, het verwijderen van eiwitten uit NR, ofwel eiwit-vrij Natural Rubber (DPNR), resulteert in een kleinere invloed van 
de lossings-temperatuur en meer constante mechanische eigenschappen. De dynamische eigenschappen kunnen worden verbeterd door de silica-silaan-rubber koppeling, zoals blijkt uit een lagere tan delta bij $60^{\circ} \mathrm{C}$ in DPNR-silica-TESPT compounds.

Coupling agents en eiwitten vertonen een ingewikkeld antagonistisch effect op de silica versterking van NR, zoals beschreven in Hoofdstuk 4. Het effect van eiwitten is het meest uitgesproken wanneer er geen silaan wordt gebruikt in NR-silica compounds. Wanneer grote hoeveelheden eiwitten in NR aanwezig zijn, zoals in skim rubber, wordt een lager Payne-effect waargenomen, vergelijkbaar met silaan-bevattende compounds. Dit toont aan, dat de interacties tussen eiwitten en silica het silica-silica netwerk kunnen verstoren. Voor NR en laag eiwit-houdende rubber DPNR, wordt de silanisering van silica verbeterd door bij voldoende hoge temperaturen te mengen. Echter, de mengtemperatuur blijkt weinig invloed te hebben op de eigenschappen van een hoog eiwit-houdend skim rubber compound. Dit toont aan, dat de hydrofobering van het silica oppervlak door silaan gedeeltelijk wordt verhinderd door silica-eiwit interacties.

De invloed van eiwitten op silica-gevulde NR is verder uitgewerkt in Hoofdstuk 5. TEM Network Visualisation van vulcanisaten zonder silaan, toont vacuolen rond de silica deeltjes en aggregaten, die duiden op zwakke vulstof-rubber interacties. Dit wordt verder ondersteund door alleen fysisch gebonden rubber in de compounds zonder silaan. In NR en DPNR vulcanisaten zonder silaan dragen sterke vulstof-vulstof interacties bij aan fysisch gebonden rubber, zoals waargenomen aan een hoger Payne effect. Daarentegen vertoont gevulkaniseerde skim rubber een lager Payne effect vanwege verstoring van het silica-silica netwerk door eiwitten. Hoewel de eiwitten het silica oppervlak hydrofoob kunnen maken en de vulstof-vulstof interactie verlagen, dragen zij niet bij aan sterke rubber-vulstof interacties, aangezien de silica-eiwit-interactie slechts leidt tot de vorming van fysisch gebonden rubber. In vergelijking worden de vacuolen duidelijker waargenomen in DPNR en in de skim rubbervulcanisaten zonder silaan, dan in het NR-vulcanisaat. De rubber-vulstof interactie van gevulkaniseerde silica-gevulde NR is sterk verbeterd door gebruik van silaan TESPT als coupling agent. De TEM Network Visualisation van de silica-vulcanisaten met TESPT toont geen vorming van vacuolen, hetgeen een sterke hechting van het rubber-netwerk aan het silica aggregaten aantoont. Dit is het resultaat van een chemische reactie tussen silica en TESPT, zoals ook blijkt uit het hoog chemisch gebonden "Bound Rubber" gehalte en een laag Payne effect. De resultaten van de TEM Network Visualisation en verschuiving in de glasovergangs-temperatuur bevestigen een sterke rubber-vulstof interactie in de silicacompounds met silaan en een minimale invloed van het eiwit in aanwezigheid van silaan. 
Vulstof-vulstof interacties in silica compounds zijn gerelateerd aan de dispersie morfologie. In Hoofdstuk 6 wordt een beter inzicht verkregen in de vulstof-vulstof interactie uit de micro-en nano-dispersie van silica, zoals verkregen door AFM- en TEM-metingen. De resultaten tonen aan, dat het gebruik van de coupling agent TESPT de micro-dispersie van silica verbetert, hetgeen resulteert in een toename van vulstof-rubber interacties. In aanwezigheid van TESPT silaan coupling agent zijn de primaire deeltjes van silica van 50 $\mathrm{nm}$ en silica-aggregaten ter grootte van $100 \mathrm{~nm}$ duidelijk zichtbaar in NR en DPNR vulcanisaten. Een gebonden rubberlaag wordt ook waargenomen op de primaire silicadeeltjes in het DPNR-TESPT vulcanisaat. Bovendien zijn de silica-aggregaten in DPNR iets beter homogeen gedispergeerd dan in NR. Dit betekent een goede micro-dispersie van silica in DPNR-TESPT, welke goed correleert met het lagere Payne effect van het vulkanisaat. Het silica-netwerk is beduidend sterker in skim rubber, zowel in afwezigheid als in aanwezigheid van een silaan coupling agent. De interactie tussen silica en eiwitten in gevulkaniseerde skim rubber wordt verder geïllustreerd door de TEM nano-dispersie, waarin grote eiwit globules ter grootte van ongeveer $300 \mathrm{~nm}$ een goede nano-dispersie van de silica aggregaten blokkeren; mogelijk ook door preferente adsorptie van het coupling agent TESPT.

Het mechanisme van silica-eiwit interactie in vergelijking met silica-silaan interactie bij hydrofobering van silica oppervlakken wordt geïllustreerd in Hoofdstuk 7. De kinetiek van de silanisering van silica is sneller in IR dan in NR, zoals blijkt uit de lagere activeringsenergie van de eerste. Een onderzoek naar het effect van NR serum-eiwitten in rubber toont aan, dat silica-eiwit interacties leiden tot een verminderde vulstof-vulstof interactie en daarmee de uitvlokking van silica onderdrukken. Echter, wanneer zowel TESPT in combinatie met eiwitten worden toegepast on een silica compound, concurreren zij met elkaar op het silica oppervlak. Het concurrentie-effect van het eiwit en TESPT om het silica-oppervlak te hydrophoberen wordt weerspiegeld in een hoger Payne effect. Het mechanisme van eiwitten op het gehydrofobeerde oppervlak van silica wordt verder aangetoond met gebruikmaking van een vlak glas-oppervlak en vergeleken met silica poeder. De contacthoek meting toont een sterk hydrofoberings-effect van NR serum-eiwitten op het silica oppervlak, waarbij de waarde dichtbij $90^{\circ}$ ligt.

In het tweede deel van dit proefschrift worden verschillende alternatieven behandeld voor het verbeteren van silica versterking in NR. In Hoofdstuk 8, wordt het gebruik van voorbehandelde of oppervlakte-gemodificeerd silica om NR te versterken behandeld. Het gebruik van oppervlakte-gemodificeerde silica elimineert de noodzaak van een coupling agent tijdens het mengen. Het commerciëel geproduceerde oppervlakte- 
gemodificeerde geprecipiteerde silica: Agilon $^{\mathrm{TM}} 400$, is een mercapto-organometallisch en mer een niet-coupling-agent behandelde silica. Het gemodificeerde silica wordt gemakkelijk opgenomen in NR tijdens het mengen aangezien het silica oppervlak al gesilaneerd is, en heeft een hogere hydrofobiciteit, hetgeen de verdraagzaamheid met NR verbetert. Het gebruik van gemodificeerde silica-verbetert de macro-dispersie van silica in NR en voorkomt silica uitvlokking vooral bij hogere concentraties silica. Het verhogen van de gemodificeeresilica concentratie in zowel NR- als DPNR-compounds verhoogt het maximale koppel in de vulcanisatie-curve, de Mooney viscositeit en het Payne effect. Door gebruikmaking van ATR-FTIR spectroscopie kan de hoeveelheid gemodificeerde-silica in het compound worden waargenomen de locatie van de piek-absorptie in het silica-gebied te meten tussen 1250 $1000 \mathrm{~cm}^{-1}$. De pieken van silica-absorptie in zowel NR als DPNR vulcanisaten verschuiven naar een lager golfgetal met toenemende gemodificeerd-silica concentraties. Bij verhoging van gemodificeerde-silica concentratie met 10 phr daalt de piek van het silica-gebied met $3,5 \mathrm{~cm}^{-1}$. De resultaten tonen aan dat er geen belangrijk verschil bestaat tussen de mechanische eigenschappen van NR en DPNR vulkanisaten gevuld met gemodificeerdesilica. De treksterkte, rek bij breuk en versterkings-index M300/M100 nemen af met toenemende silica concentratie. De toename van de tan delta bij $60^{\circ} \mathrm{C}$ en het hysteretisch energieverlies voor zowel NR als DPNR vulcanisaten is evenredig met de hoeveelheid van het gemodificeerde-silica toegepast. In vergelijking met in-situ TESPT-behandelde silica, is de gemodificeerde- silica significant beter in tan delta bij $60{ }^{\circ} \mathrm{C}$ voor NR, hoewel er voor DPNR geen verschil wordt waargenomen.

De keuze van het elastomeer met een optimale $\mathrm{Tg}$, of de juiste combinatie van een mengsel van elastomeren, spelen een belangrijke rol bij het bereiken van een compromis tussen de vele eisen te stellen aan een autoband. Hoofdstuk 9 richt zich op de versterking van een silica-silaan-systeem in NR, door gebruik te maken rubber mengsels van NR / IR en NR / BR. IR en BR zijn geselecteerd vanwege een $10 \%$ vinyl-gehalte. Het mengsel van NR met BR is bedoeld om de slijtvastheid van een NR-gebaseerde rubbersamenstelling verbeteren. Bovendien, door NR met vinyl-IR te mengen moet de reactiviteit van de silicasilaan-rubber koppeling verbeterd worden. De Tg van het NR / IR mengsel vertoont een verschuiving vmet de hoeveelheid $10 \%$-vinyl IR in het NR / IR mengsel, hetgeen zich laat vertalen in een daling van de slijtvastheid en flexibiliteit bij lage temperatuur van het NR / IR mengsel. De NR / IR mengsels verbeteren duidelijk in slip-weerstand, maar geven een slechte rolweerstand, zoals blijkt uit de hogere tan delta in het bereik van 0 tot $30{ }^{\circ} \mathrm{C}$ en eveneens hogere tan delta in het bereik van 30 tot $70^{\circ} \mathrm{C}$. Een afname in $\mathrm{Tg}$ van de NR/BR mengsels wordt waargenomen bij toenemende $10 \%$ vinyl-BR hoeveelheid. De NR / BR 
combinatie vertoont superieure slijtvastheid ten opzichte van NR. Aan de andere kant is de natte slip-weerstand van de NR / BR mengsels iets beter dan voor NR. Er wordt echter een hogere tan delta waarde waargenomen tussen 30 tot $70^{\circ} \mathrm{C}$, hetgeen aan aanwijzing is voor slechtere rolweerstand voor de NR / BR mengsels.

Het 80/10/10 mengsel van NR / BR / IR heeft een hogere $\operatorname{Tg}\left(-43^{\circ} \mathrm{C}\right)$ dan NR $(-48$ $\left.{ }^{\circ} \mathrm{C}\right)$. Het NR / BR / IR mengsel heeft duidelijke superioriteit t.a.v. grip op nat wegdek en de remprestaties, zoals blijkt uit de hogere tan delta waarde in het bereik tussen $0-30{ }^{\circ} \mathrm{C}$. Aan de andere kant geeft het 80/10/10 NR / BR / IR mengsel geen verbetering van de slijtvastheid en de lage temperatuurflexibiliteit. Daarnaast vertoont het mengsel NR / IR / BR ook ongunstige rolweerstand en warmteontwikkeling. De resultaten tonen aan, dat de toepassing van kleine hoeveelheden vinyl-groepen via IR of $B R, 1,5 \%$, een aanzienlijke wijziging opleveren in dynamische eigenschappen van de compounds. Dit is vooral te zien in de natte grip-eigenschappen. Optimalisatie van de loopvlak eigenschappen door polymeren te mengen is nog lang niet voldoende om de magische driehoek uit te breiden. De vier elementen in silica technologie: het polymeer, de silica, de coupling agent en de mengomstandigheden, moeten goed worden geoptimaliseerd om een positief compromis te bereiken tussen de diverse band-eigenschappen.

Naast de silaniserings-reactie, die al in de vorige hoofdstukken is onderzocht, kunnen voortijdige vernettings-reacties plaatsvinden tijdens het mengen van NR-silicaTESPT compounds, doordat er zwavel vrijkomt uit TESPT. Vandaar dat in de bijlag de rol van TESPT als zwavel-donor in het vulkaniseren van rubber geïllustreerd wordt in een beperkte model-compound vulkanisatie studie. De reactie van TESPT met Tetra-MethylEtheen (TME) produceert verknoopte producten met een structuur van TME-S - TME, zelfs in afwezigheid van vulkanisatieversnellers. Interessant is, dat zowel TESPT-TME en TMETESPD reactieproducten een vergelijkbare structuur van verknoopte producten hebben met een polysulfidebrug. Het mechanisme van de vorming van verknoopte producten uit de zwavel vrijgekomen uit TESPT wordt aangetoond.

Algemeen kan worden gesteld, dat de versterking van NR met silica een hele uitdaging is. In de silica-technologie is de gecontroleerde silaniserings-reactie tussen silica en silaan een kritisch punt. Echter, NR is intrinsiek minder thermisch stabiel dan synthetische elastomeren, terwijl vrij hoge temperaturen en lange mengtijden nodig zijn voor een coupling agent om in eerste instantie met de silica en later met rubber polymeren te reageren. Bovendien is NR is een natuurproduct, onderhevig aan seizoens-schommelingen. De invloed van niet-rubber bestanddelen op silica versterking in NR is onderzocht in dit proefschrift. Het effect van eiwitten is het meest uitgesproken bij een eiwit-gehalte of 
wanneer geen silaan wordt gebruikt in het compound. Er is sprake van onderlinge concurrentie tussen silaan en eiwitten, indien aanwezig in vergelijkbare hoeveelheden, bij de hydrofobering van het silica oppervlak. Anderzijds, het gebruik van DPNR bij een silicasilaan systeem geeft verbetering van de dynamische en mechanische eigenschappen, evenals de tan delta bij $60^{\circ} \mathrm{C}$ van de vulkanisaten. Niettemin zal de combinatie van silica technologie met NR milieuvriendelijker banden produceren en aanleiding geven voor tal van nieuwe en spannende onderzoeken op het gebied van silica-technologie voor NR.

\section{SUGGESTIES VOOR VERDER ONDERZOEK}

Dit proefschrift geeft inzicht in de vele aspecten van NR voor gebruik in banden loopvlakken gebaseerd op silica versterking. Mogen de resultaten van dit onderzoek een gids en uitgangspunt zijn voor verdere studies over silica-technologie voor NR en over NR zelf.

Verschillende soorten natuurlijke rubbers zijn onderzocht in dit proefschrift. Wat interessant is, zijn de goede eigenschappen van DPNR, aangezien dit meer consistente fysische eigenschappen geeft in vergelijking met reguliere NR. Fundamenteel onderzoek naar de netwerkstructuur van DPNR kan bijdragen een verdere verbetering van de eigenschappen. Bovendien is er de potentie voor gebruik van DPNR voor silica-silaan systemen voor banden-loopvlakken. Vandaar dat optimalisatie van de parameters voor de silica-TESPT-DPNR compounds, qua formulering en mengomstandigheden verder uitgewerkt zou moeten worden.

Het werk beschreven in dit proefschrift is geconcentreerd geweest op TESPT als coupling agent. Onderzoek naar verschillende typen coupling agents op silica-versterkte $\mathrm{NR}$, is een onderwerp dat nader uitgevoerd moet worden. Diverse coupling agents op basis van zwavelhoudende silanen, zoals mercapto-silanen en geblokkeerde silanen, alsmede zwavelvrije silanen, kunnen geschikt gemaakt worden voor NR mogelijk te maken, waarvoor diepgaander onderzoek vereist is.

Het sterke hydrofoberings-effect van eiwitten op het silica oppervlak is zeer fascinerend. Met het voordeel van eiwit-engineering, zou het interessant zijn om eiwitten zoals NR serum te gebruiken als "compatilizers" (verdraagzaamheid bevorderende middelen) voor versterking van NR met silica-bevattende vulstoffen, in compounds voor niet-banden toepassingen. 


\section{Ringkasan dan Pandangan}

\section{RINGKASAN}

Pemilihan empat elemen penting dalam teknologi silika iaitu polimer getah, jenis silika, agen pengikat yang berkesan dan teknologi penyebatian yang sesuai, adalah peting untuk mengembangkan segitiga ajaib bagi teknologi tayar: iaitu kompromi di antara rintangan guling, cengkaman basah dan kehausan. Kecenderungan masakini dalam penggunaan bahan mentah berasaskan elastomer yang mesra alam menjadi pencetus kepada penyelidikan berkenaan dan peningkatan penggunaan getah asli (NR). Kajian semula secara ekstensif dalam Bab 2 mendapati silika mempunyai kesan positif terhadap penurunan rintangan guling dan pengurangan pengumpulan haba dalam tayar berbanding dengan hitam karbon dalam sebatian NR. Faktor kritikal dalam teknologi silika ialah gandingan pada sekala nano di antara molekul getah dan permukaan silika dengan agen pengikat, tidak diganggu oleh agen aktif permukaan lain seperti protein. Walau bagaimanapun, hanya sedikit maklumat berkenaan kesan unsur bukan getah, terutamanya protein ke atas sebatian getah berpengisi silika yang diketahui. Oleh itu, di dalam bahagian pertama tesis ini, peranan unsur bukan getah, terutamanya protein, ke atas interaksi silikasilika dan silika-silana telah dikaji untuk memahami mekanisma penguatan silika dalam sebatian NR.

Elemen penting dalam penguatan getah menggunakan silika ialah ikatan kimia di antara silika dan getah, di mana ianya diguna pakai untuk getah sintetik dan asli. Kajian dalam Bab 3 menunjukkan perkembangan suhu adalah parameter penting dalam penyebatian silika dan getah asli atau sintetik poliisoprena (IR) dengan bantuan bis(trietoksisililpropil)-tetrasulfida (TESPT) sebagai agen pengikat untuk mencapai silanisasi silika yang dikehendaki. Interaksi silika-silika berkurangan dengan perubahan permukaan silika menjadi lebih hidrofobia menerusi silanisasi TESPT pada suhu yang tinggi dan bersesuaian. Dalam sebatian NR, tindakbalas silanisasi di antara silika dan TESPT bertambah dengan peningkatan suhu akhir penyebatian. Sekiranya suhu akhir penyebatian melebihi optimum, $150^{\circ} \mathrm{C}$, NR mengalami penurunan dalam sifat-sifat dinamik dan mekanikal yang dijangkakan oleh degradasi termo-mekanikal. Kesan ini lebih ketara pada getah sintetik IR. Sebaliknya dengan penyahan protein dari NR seperti getah asli ternyah protein, (DPNR), ianya hanya memberi sedikit kesan pada suhu akhir penyebatian dan 
memberikan sifat-sifat mekanikal yang lebih malar. Sifat-sifat dinamik bertambah baik dengan gandingan silika-silana-getah ini dibuktikan dengan nilai $\tan \delta$ pada $60^{\circ} \mathrm{C}$ yang rendah terutamanya pada sebatian DPNR-silika-TESPT.

Agen pengikat dan protein menunjukkan kesan antagonistik yang kompleks bagi penguatan silika dalam getah asli seperti yang ditunjukkan dalam Bab 4. Kesan protein lebih ketara apabila silana tidak digunakan dalam sebatian silika-getah asli. Apabila kandungan protein dalam getah asli tinggi seperti dalam getah skim, kesan Payne yang rendah dapat dilihat di mana ianya sebanding dengan sebatian mengandungi silana. Ini menunjukkan bahawa interaksi di antara protein dan silika dapat mengganggu rangkaian silika-silika. Bagi getah asli dan getah dengan kandungan protein rendah DPNR, silanisasi silika meningkat pada suhu penyebatian yang cukup tinggi. Bagaimanapun, suhu penyebatian tidak memberikan kesan ke atas sebatian getah skim yang berkandungan protein tinggi. Ini menunjukkan pemhidrofobiaan permukaan silika dengan silana berkemungkinan separa terhalang disebabkan interaksi silika dan protein.

Kesan protein ke atas sebatian NR berpengisi silika dikaji lebih terperinci dalam Bab 5. Teknik Pengvisualan Rangkaian melalui Mikroskopi Elektron Transmisi (TEM) ke atas vulkanizat tanpa silana memperlihatkan vakuol di sekeliling zarah dan agregat silika, di mana ianya menunujukkan interaksi pengisi dan getah yang lemah. Ini disokong dengan hanya getah terikat fizikal yang terdapat pada sebatian tanpa silana. Bagi vulkanizat NR dan DPNR tanpa silana, interaksi antara pengisi yang kuat seperti yang jelas dilihat melalui kesan Payne yang tinggi menyumbang kepada pembentukkan getah terikat fizikal. Ini berbeza dengan getah skim yang mempunyai kesan Payne yang rendah disebabkan rangkaian silika telah terganggu oleh protein. Walaupun protein mengubah permukaan silika menjadi lebih hidrofobia dan mengurangkan interaksi antara pengisi, tetapi ia tidak menyumbang kepada peningkatan interaksi antara getah dan pengisi, di mana interaksi antara silika dan protein hanya membentuk getah terikat fizikal. Sebagai perbandingan, vakuol jelas kelihatan dalam vulkanizat DPNR dan getah skim tanpa silana daripada vulkanizat NR. Interaksi getah-pengisi dalam vulkanizat NR berpengisi silika bertambah baik dengan menggunakan silana TESPT sebagai agen pengikat. Pengvisualan Rangkaian melalui TEM ke atas vulkanizat silika-TESPT memperlihatkan tiada vakuol yang kelihatan, dan ini menunjukkan pengikatan rangkaian getah yang kuat ke atas aggregat silika. Ini adalah hasil daripada tindak balas kimia di antara silika dan TESPT, yang juga ditunjukkan melalui getah terikat kimia yang tinggi dan kesan Payne yang rendah. Kajian dari Pengvisualan Rangkaian melalui TEM dan anjakan pada suhu peralihan kaca $(\mathrm{Tg})$ 
mengesahkan interaksi antara getah dan pengisi yang kuat dalam sebatian getah menggunakan silana dan kesan protein yang lemah dengan kehadiran silana.

Interaksi pengisi-pengisi dalam sebatian silika berhubung kait dengan morfologi serakan. Dalam Bab 6, pemahaman yang lebih baik ke atas interaksi antara pengisi dapat dicapai melalui serakan silika peringkat mikro dan nano menggunakan pengukuran Mikroskopi Daya Atom (AFM) and TEM. Kajian menunjukkan penggunaan agen pengikat TESPT memperbaiki serakan silika peringkat mikro, di mana ianya meningkatkan interaksi getah-pengisi. Dengan kehadiran agen pengikat silana TESPT, zarah utama silika bersaiz $50 \mathrm{~nm}$ dan aggregate bersaiz $100 \mathrm{~nm}$ dapat dilihat dengan jelas dalam vulkanizat NR dan DPNR. Lapisan getah terikat juga dapat dilihat pada zarah utama silika dalam vulkanizat DPNR-TESPT. Di samping itu, aggregat silika dalam vulkanizat DPNR berserak lebih seragam berbanding dengan vulkanizat NR, menunjukkan serakan silika sekala mikro yang baik, yang berhubung-kait dengan kesan Payne yang rendah bagi vulkanizat DPNR-TESPT. Rangkaian silika dilihat sangat kuat dalam getah skim, tanpa atau dengan kehadiran agen pengikat silana. Interaksi silika-protein dalam vulkanizat getah skim dapat dilihat melalui TEM serakan sekala nano, di mana globul protein yang besar bersaiz anggaran $300 \mathrm{~nm}$ menghalangi serakan-nano agregat silika, berkemungkinan juga disebabkan oleh pilihan penjerapan oleh agen pengikat TESPT.

Mekanisma interaksi silika-protein berbanding dengan interaksi silika-silana dalam penghidrofobiaan permukaan silika dijelaskan dalam Bab 7. Kinetik silanisasi bagi silika lebih pantas bagi getah sintetik IR berbanding getah asli NR seperti yang ditunjukkan oleh tenaga pengaktifan yang rendah. Kajian berkenaan kesan protein serum NR menunjukkan interaksi silika-protein mengurangkan interaksi pengisi-pengisi dan menahan flokulasi silica. Walau bagaimanapun, apabila kombinasi kedua-dua TESPT dan protein digunakan dalam sebatian silika, mereka bersaing untuk mendapatkan permukaan silika. Kesan persaingan antara protein dan TESPT dalam penghidrofobiaan permukaan silika ini digambarkan dengan kesan Payne yang tinggi. Mekanisma protein dalam penghidrofobiaan permukaan silika ini ditunjukkan lebih lanjut dengan menggunakan permukaan gelas yang rata dan dibandingkan dengan serbuk silika. Pengukuran sudut sentuhan menunjukkan kesan hidrofobia yang tinggi oleh protein serum NR ke atas permukaan silika, di mana nilainya hampir kepada 90‥

Bahagian kedua dalam tesis ini membincangkan alternatif yang berlainan dalam menghadapi cabaran untuk memperbaiki penguatan silika dalam NR. Dalam Bab 8, penggunaan silika yang telah dirawat atau permukaan terubah suai untuk menguatkan NR telah dikaji. Pengunaan silika terubah-suai permukaan menghapuskan keperluan 
agen pengikat semasa penyebatian. Silika termendak terubai-suai permukaan komersil, Agilon $^{\text {TM }} 400$ ialah silika yang telah dirawat dengan organologam merkapto dan agen bukan pengikat. Silika terubah suai ini senang disebatikan ke dalam NR semasa penyebatian kerana silika tersebut telah disilanasasikan dan mempunyai kehidrofobiaan yang tinggi yang mana ianya meningkatkan keserasian dengan NR. Penggunaan silika terubah suai memperbaiki penyerakan mikro silika dalam NR dan mencegah flokulasi silika terutamanya pada tahap bebanan silika yang tinggi. Peningkatan bebanan silika terubah suai dalam kedua-dua sebatian NR dan DPNR meningkatkan kilas maxima pada lengkung pemvulkan, kelikatan Mooney dan kesan Payne. Dengan menggunakan spektroskopi ATR-FTIR, jumlah silika terubah suai dalam sebatian boleh diawasi dengan puncak penyerapan pada rantau silika di antara 1250-1000 $\mathrm{cm}^{-1}$. Puncak penyerapan silika bagi kedua-dua vulkanizat NR dan DPNR berubah ke arah nombor gelombang yang lebih rendah dengan peningkatan bebanan silika terubah suai. Untuk peningkatan silika terubah suai sebanyak 10 phr, puncak nombor gelombang menurun sebanyak $3.5 \mathrm{~cm}^{-1}$. Kajian menunjukkan tiada perbezaan pada sifat-sifat mekanikal di antara vulkanizat NR dan DPNR berpengisi silika terubah suai. Kekuatan regangan, pemanjangan tahap putus dan index kekuatan iaitu nisbah M300/M100 menurun dengan peningkatan bebanan. Peningkatan tan delta pada $60^{\circ} \mathrm{C}$ dan tenaga histerisis yang hilang pada kedua-dua vulkanizat NR dan DPNR adalah berkadar dengan jumlah penggunaan silika terubah suai. Sebagai perbandingan dengan silika yang dirawat dengan TESPT secara in situ, silika terubah suai menunjukkan penambahbaikan yang nyata pada tan delta di $60^{\circ} \mathrm{C}$ untuk NR. Walaubagaimanapun, tiada perbezaan dapat dilihat bagi DPNR..

Pilihan elastomer yang mempunyai suhu peralihan kaca yang optima atau kombinasi campuran elastomer yang sesuai, memainkan faktor utama dalam mendapatkan kompromi di antara keperluan-keperluan bagi tayar. Bab 9 memberikan fokus kepada penguatan sistem silika dan silana dalam NR menggunakan campuran NR/IR dan NR/BR. Kedua-dua getah IR dan BR dipilih untuk mempunyai kandungan vinil sebanyak $10 \%$. Campuran NR dan BR adalah salah satu langkah untuk memperbaiki ketahanan kelelasan sebatian telapak tayar berasaskan getah asli. Di samping itu, campuran NR dan IR-vinil adalah untuk memperbaiki reaktiviti ikatan silika-silana-getah. Tg bagi campuran NR/IR menunjukkan anjakan dengan kandungan IR dalam campuran NR/IR dimana ianya diterjemahkan kepada penurunan dalam rintangan kelelasan dan kelenturan pada suhu rendah bagi campuran ini. Campuran NR/IR dengan jelas memperbaiki rintangan gelincir tetapi memberikan rintangan guling yang buruk seperti yang ditunjukkan masing-masing oleh kenaikan tan delta pada suhu $0-30^{\circ} \mathrm{C}$ dan $30-70^{\circ} \mathrm{C}$. Penurunan $\mathrm{Tg}$ bagi campuran 
NR/BR dilihat dengan peningkatan nisbah BR-vinil. Campuran NR/BR memiliki rintangan lelasan yang unggul berbanding dengan getah NR. Di samping itu, rintangan gelincir basah bagi campuran NR/BR adalah lebih baik daripada NR. Walau bagaimanapun, nilai tan delta yang tinggi di lihat pada julat $30-70^{\circ} \mathrm{C}$, di mana ianya memberikan indikasi rintangan guling yang kurang bagi campuran NR/BR.

Campuran NR/BR/IR 80/10/10 mempunyai Tg yang lebih tinggi $\left(-43^{\circ} \mathrm{C}\right)$ berbanding dengan NR $\left(-48^{\circ} \mathrm{C}\right)$. Campuran NR/BR/IR jelas memiliki rintangan gelincir basah dan prestasi pembrekan yang lebih tinggi seperti yang ditunjukkan dari nilai tan delta pada julat 0-30ㄷ․ Sebaliknya, campuran NR/BR/IR 80/10/10 tidak memberikan penambahbaikan pada rintangan lelasan dan kelenturan pada suhu rendah. Di samping itu, campuran $\mathrm{NR} / \mathrm{R} / \mathrm{BR}$ juga mempunyai rintangan guling dan pengumpulan haba yang tidak memuaskan. Kajian menunjukkan penggunaan kandungan vinil dalam jumlah yang kecil melalui IR atau BR, sebanyak 1-5\%, menyebabkan perubahan yang besar dalam sifat dinamik sebatian campuran, terutamanya dalam sifat gelincir basah. Pengoptimuman sifat telapak tayar menggunakan campuran polimer adalah tidak mencukupi untuk mengembangkan segitiga ajaib. Keempat-empat elemen dalam teknologi silika iaitu polimer, silika, agen pengikat dan keadaan penyebatian perlu dioptimumkan untuk mencapai kompromi yang positif ke atas sifat-sifat tayar.

Selain daripada reaksi silanisasi, yang telah dikaji dalam bab-bab yang lepas, reaksi pra-sambung silang boleh berlaku semasa penyebatian sebatian NR-silica-TESPT disebabkan sulfur yang dikeluarkan daripada TESPT. Oleh itu, dalam Lampiran, peranan TESPT sebagai penderma sulfur dalam pemvulkanan getah ditunjukkan dalam kajian model pemvulkanan yang terbatas. Reaksi di antara TESPT dan Tetra metil etilena (TME) menghasilkan produk sambung silang dengan struktur TME-Sx-TME, walaupun tanpa sebarang agen pencepat pemvulkanan. Lebih menarik, produk untuk kedua-dua reaksi TESPT-TME dan TESPD-TME mempunyai struktur produk sambung silang dengan rangkain polisulfidik yang sama. Mekanisma pembentukan produk sambung silang daripada sulfur yang dikeluarkan oleh TESPT ditunjukkan.

Keseluruhannya, penguatan silika dalam getah asli adalah satu cabaran. Dalam teknologi silika, reaksi silanisasi yang terkawal di antara silika dan silana adalah sangat penting. Walau bagaimanapun, NR adalah kurang stabil dari segi terma jika dibandingkan dengan elastomer sintetik, di mana suhu yang tinggi dan masa yang panjang diperlukan untuk agen pengikat bertindak pada awalnya dengan silika dan pada akhirnya dengan getah polimer. Di samping itu, NR adalah produk semulajadi yang bergantung kepada variasi bermusim. Pengaruh unsur bukan getah kepada penguatan silika dalam getah asli telah 
dikaji dalam tesis ini. Kesan protein sangat ketara pada jumlah yang tinggi dan tiada silana digunakan dalam sebatian. Terdapat kesan persaingan di antara silana dan protein apabila digunakan pada kuantiti yang sama dalam memhidrofobiaan permukaan silika. Pada sudut yang lain, penggunaan DPNR untuk sistem silika-silana memberikan penambahbaikan dalam sifat-sifat dinamik dan mekanikal, dan juga tan delta pada $60^{\circ} \mathrm{C}$ bagi vulkanizat tersebut. Walau bagaimanapun, kombinasi teknologi silika dan NR akan menghasilkan tayar yang lebih mesra alam, dan membuka ruang kepada banyak lagi kajian yang baru dan menarik dalam teknologi silika untuk NR.

\section{Cadangan untuk Penyelidikan Lanjut}

Tesis ini memberikan pengertian mendalam ke atas banyak aspek getah asli untuk digunakan dalam sebatian telapak tayar silika berserakan tinggi. Kajian daripada tesis ini diharapkan dapat menjadi panduan dan juga permulaan untuk kajian lanjut dalam teknologi silika untuk getah asli, dan juga untuk getah asli itu sendiri.

Getah asli berlainan jenis telah dikaji dalam tesis ini. Apa yang menarik ialah sifatsifat getah DPNR yang baik, di mana ianya memberikan sifat-sifat fizikal yang stabil jika dibandingkan dengan getah asli biasa. Kajian asas berkenaan struktur rangkaian DPNR yang memberikan sifat-sifat yang unggul adalah amat menarik perhatian. Di samping itu, terdapat potensi untuk DPNR digunakan dalam sistem silika-silana untuk telapak tayar. Oleh itu, pemoptimuman parameter bagi sebatian silika-TESPT-DPNR terutamanya dari segi formulasi dan keadaan pencampuran harus dikaji lebih kanjut.

Kajian yang dibahaskan dalam tesis ini hanya berlegar kepada agen pengikat TESPT. Kajian berkenaan agen pengikat yang berlainan jenis ke atas sebatian getah asli berpenguatan silika adalah menjadi topik untuk kajian lanjut. Berbagai jenis agen pengikat daripada silana mengandungi sulfur, dan juga silana tanpa sulfur boleh dioptimakan untuk kesesuaian dengan NR dan kajian yang lebih lanjut perlu dijalankan.

Kesan pemhidrofobiaan protein yang tinggi ke atas permukaan silika amat menarik. Dengan kelebihan kejuruteraan protein, ianya amat menarik untuk menggunakan protein seperti protein serum NR sebagai agen penyesuaian bagi penguatan getah asli dengan pengisi yang mempunyai kandungan silika dalam sebatian untuk aplikasi bukan tayar. 


\section{Symbols and Abbreviations}

$\begin{array}{ll}\text { SYMBOL } & \text { DESCRIPTION } \\ \gamma_{s} & \text { surface energy of solid } \\ \gamma_{s}^{d} & \text { dispersive component of surface energy } \\ \gamma_{s}^{s p} & \text { specific component of surface energy } \\ \alpha_{f} & \text { Wolff's filler structure parameter or in-rubber structure } \\ v_{r o} & \text { volume fraction of rubber in unfilled (gum) vulcanizate } \\ v_{r f} & \text { volume fraction of rubber in the filled vulcanizate } \\ \phi & \text { volume fraction of filler in the filled vulcanizate } \\ \eta^{*} & \text { complex viscosity } \\ \Delta T_{\text {centre }} & \text { heat build up } \\ 1 / R T & \text { reciprocal of the product of gas constant }\left(8.314 \mathrm{JK}^{-1} \mathrm{~mol}^{-1} \text { ) and absolute }\right. \\ \text { G' } & \text { temperature (in Kelvin) } \\ \mathrm{G}^{\prime \prime} & \text { storage modulus } \\ \text { M100 } & \text { loss modulus } \\ \text { M300 } & \text { Tensile modulus at } 100 \% \text { elongation } \\ \text { M300/M100 } & \text { Tensile modulus at 300\% elongation } \\ \text { Q } & \text { Reinforcement index } \\ \text { Tan } \delta & \text { swelling value } \\ & \text { loss angle }\end{array}$

\section{ABbreViation Description}

ACN

Acetonitrile

AFM

Atomic Force Microscopy

ATR-FTIR

Attenuated Total Reflection Fourier Transform Infrared

BET

Brunauer-Emmett-Teller nitrogen-adsorption

BPC

Base peak chromatogram

$\mathrm{BR}$

Butadiene rubber

BRC

Bound rubber content

CB

Carbon black 
CBS

$\mathrm{CO}_{2}$

DBPA

DEG

DMA

DPG

DPNR

DT

ENR

EB

EC

EG

EV

EM

EPDM

eSBR

FTIR

HD

HPLC

IGC

IR

IRSG

LCMS-ESI

LNR

MBS

MCV

MG

$\mathrm{ML}(1+4)$

MS (2+4)

$\mathrm{N}_{2} \mathrm{SA}$

NR

ODPA
$\mathrm{N}$-cyclohexyl-2-benzothiazyl sulfenamide

Carbon dioxide

Dibutylphthalate adsorption

Diethylene glycol

Dynamic mechanical analyzer

Diphenyl guanidine

Deproteinized natural rubber

Dump temperature

Epoxidized natural rubber

Elongation at break

Equilibrium cure system

Ethylene glycol

Efficient system vulcanization

Earth mover

Ethylene propylene diene rubber

emulsion styrene butadiene rubber

Fourier transform infrared spectroscopy

Highly dispersible

High performance liquid chromatography

Inverse gas chromatography

Polyisoprene

International Rubber Study Group

Liquid chromatography mass spectroscopy with electronspray ionization

Liquid natural rubber

Morpholinothio benzothiazole

Model compound vulcanization

Methyl methacrylate grafted rubber

Mooney viscosity after 1 minute of preheating and 4 minutes of test time measured using a large rotor

Mooney viscosity after 2 minute of preheating and 4 minutes of test time measured using a small rotor

Nitrogen-adsorption surface area

Natural rubber

Octylated diphenylamine 


\begin{tabular}{ll} 
PEG & Polyethylene glycol \\
phr & Parts per hundred rubber \\
RPA & Rubber process analyzer \\
RSS & Ribbed smoked sheet \\
RT & Room temperature \\
S & Sulfur \\
SBR & Styrene butadiene rubber \\
SBR & Solution butadiene rubber \\
SEM & Scanning Electron Microscopy \\
SIR & Standard Indonesian Rubber \\
SMR & Standard Malaysian Rubber \\
SSBR & Solution styrene butadiene rubber \\
StA & Stearic acid \\
TARRC & Tun Abdul Razak Research Centre \\
TDAE & Treated distillate aromatic extract oil \\
TEA & Triethanolamine \\
TEM & Transmission Electron Microscopy \\
TESPD & Bis (triethoxysilylpropyl) disulfide \\
TESPT & Bis (triethoxysilylpropyl) tetrasulfide \\
TGA & Thermogravimetric Analysis \\
TME & Tetra methyl ethylene or 2,3-dimethyl-2-butene \\
TMTM & Tetramethyl thiuram monosulfide \\
TMQ & 2,2,4-trimethy-1,2-dihydroquinoline \\
TS & Tensile strength \\
Tg & Glass transition temperature \\
TTS & Thai Technical Rubber \\
ZnO & Zinc oxide \\
\hline
\end{tabular}




\section{Bibliography}

\section{JoURnal ARTICLES}

1. S.S. Sarkawi, W.K. Dierkes and J.W.M. Noordermeer, Natural Rubber-Silica Combinations for Low Rolling Resistance Truck Tire Treads, Rubber World, 247(2), p.26-31 (2012).

2. S.S. Sarkawi, W.K. Dierkes and J.W.M. Noordermeer, The Effect of Protein Content in Natural Rubber on Performance of Silica Filled Compounds as Influenced by Processing Temperature, Kautschuk Gummi Kunststoffe, 66 (3),p.27-33 (2013).

3. S.S. Sarkawi, W.K. Dierkes and J.W.M. Noordermeer, The Influence of Non-Rubber Constituents on Performance of Silica Reinforced Natural Rubber Compounds, European Polymer Journal, 49, p.3199-3209 (2013).

4. S.S. Sarkawi, W.K. Dierkes and J.W.M. Noordermeer, Reinforcement of Natural Rubber by Precipitated Silica: The Influence of Processing Temperature, Rubber Chemistry and Technology Journal, in press and available online (2013).

5. S.S. Sarkawi, W. Kaewsakul, K. Sahakaro, W.K. Dierkes and J.W.M. Noordermeer, A Review on Reinforcement of Natural Rubber by Silica Fillers for Use in Low-Rolling Resistance Tyres, J. Rubber Res., submitted (2013).

6. S.S. Sarkawi, W.K. Dierkes and J.W.M. Noordermeer, Effect of Silane Coupling Agent on the Morphology of Silica Reinforced Natural Rubber Compounds, Kautschuk Gummi Kunststoffe, submitted (2013).

7. S.S. Sarkawi, W.K. Dierkes and J.W.M. Noordermeer, Morphology of Silica Reinforced Natural Rubber: The Effect of Silane Coupling Agents, Rubber Chemistry and Technology Journal, submitted (2013).

8. S.S. Sarkawi, W.K. Dierkes and J.W.M. Noordermeer, Elucidation of Filler-to-Filler and Filler-to-Rubber Interactions in Silica Reinforced Natural Rubber using TEM Network Visualization, Composite Science and Technology, in preparation (2013).

\section{MAgAZINe ARTICLE}

9. S.S. Sarkawi, W.K. Dierkes and J.W.M. Noordermeer, Reinforcement of Natural Rubber by Silica Fillers, Tire Technology International Annual Showcase 2012, p.18-22 (2012). 


\section{MONOGRAPH AND BOOK CHAPTER}

10. S.S. Sarkawi, W.K. Dierkes and J.W.M. Noordermeer, A Review on Silica Reinforcement in Natural Rubber, Malaysian Rubber Board, Monograph no.22. (2011).

11. S.S. Sarkawi, W.K. Dierkes and J.W.M. Noordermeer, Progress in Polymer Research Vol. 2, Ed. A.M. Azman, The Influence of Mixing Temperature on Silica Reinforced Natural Rubber, Polymer Research Center (PORCE), Bangi, Malaysia (2013).

\section{Conference Proceedings}

12. S.S. Sarkawi, W.K. Dierkes and J.W.M. Noordermeer, The Influence of Mixing Temperature on Silica Reinforced Natural Rubber, Proceeding of Malaysia Polymer International Conference 2011, Equitorial Bangi-Putrajaya, Malaysia, 18-20 October 2011.

13. S.S. Sarkawi, W.K. Dierkes and J.W.M. Noordermeer, Natural Rubber-Silica Combinations for Low Rolling Resistance Truck Tyre Treads, Proceeding of German Rubber Conference (DKT) 2012, Nuremberg Convention Centre, Nuremberg, Germany, 2-5 July 2012.

14. S.S. Sarkawi, W.K. Dierkes and J.W.M. Noordermeer, The Challenges of Silica-Silane Reinforcement of Natural Rubber, Proceeding of 15th International Conference 'Polymeric Materials', Halle (Saale), Germany, 12-14 September 2012.

15. S.S. Sarkawi, W.K. Dierkes and J.W.M. Noordermeer, Reinforcement of Natural Rubber by Precipitated Silica: The Influence of Processing Temperature, Fall $182^{\text {nd }}$ Technical Meeting of the Rubber Division, ACS, Cincinnati, Ohio, US, paper no.52, 9-11 October 2012

16. S.S. Sarkawi, W.K. Dierkes and J.W.M. Noordermeer, The Challenges of Silica-Silane Reinforcement of Natural Rubber, Proceeding of the Polymer Processing Society 28th Annual Meeting, Pattaya, Thailand, 11-15 December 2012.

17. S.S. Sarkawi, W.K. Dierkes and J.W.M. Noordermeer, Morphology of Silica-Reinforced Natural Rubber: The Effect of Silane Coupling Agent, Fall $184^{\text {th }}$ Technical Meeting of the Rubber Division, ACS, Cleveland, Ohio, US, paper no.86, 8-10 October 2013.

\section{External Presentations}

1. S.S. Sarkawi, J.W.M. Noordermeer and W.K. Dierkes, Energy-Saving Tyres Based on Natural Rubber, 2010 Meeting The Future of Natural Rubber, EU-PEARLS, Agropolis International, Montpellier, France, 14-15 October 2010. 
2. S.S. Sarkawi, W.K. Dierkes and J.W.M. Noordermeer, The Effect of Silane Coupling Agent, TESPT on Silica-filled Natural Rubber, PhD-workshop IPF/UT/MLU/TUL/Contitech, Hannover, 6-8 June 2011.

3. S.S. Sarkawi, W.K. Dierkes and J.W.M. Noordermeer, The Influence of Mixing Temperature on Silica Reinforced Natural Rubber, Malaysia Polymer International Conference 2011, Equitorial Bangi-Putrajaya, Malaysia, 18 - 20 October 2011.

4. S.S. Sarkawi, W.K.Dierkes and J.W.M. Noordermeer, Reinforcement of Natural Rubber by Silica Fillers, Tire Technology Expo 2012 Conference, Köln Messe, Cologne, Germany, 14-16 February 2012.

5. S.S. Sarkawi, W.K.Dierkes and J.W.M. Noordermeer, The Influence of Mixing Temperature on Silica-Reinforced Natural Rubber, Dutch Polymer Days 2012, De Werelt Conference Centre, Lunteren, the Netherlands, 12-13 March 2012.

6. S.S. Sarkawi, W.K.Dierkes and J.W.M. Noordermeer, The Effect of Protein Content in Natural Rubber on Performance of Silica Filled Compounds as Influenced by

Processing Temperature, International Rubber Conference 2012, Ramada Plaza Jeju Hotel, Jeju, South Korea, 21-24 May 2012.

7. S.S. Sarkawi, W.K.Dierkes and J.W.M. Noordermeer, Chances and Threats for Natural Rubber for Use in Low Rolling Resistance Truck Tyres, Vereniging van Kunststof en Rubber Technologen (VKRT) ECCO Meeting, Oude Tolhuys, Utrecht, the Netherlands, 13 September 2012.

8. J.W.M. Noordermeer, W.K. Dierkes, S. S. Sarkawi, W. Kaewsakul and K. Sahakaro, Chances and Threats for Natural Rubber for Use in Low Rolling Resistance Tyres, 2012 Congress : BioRubber for Europe in Global Perspective, EU-PEARLS, Wageningen, the Netherlands, 24-25 September 2012.

9. S.S. Sarkawi, W.K. Dierkes and J.W.M. Noordermeer, Reinforcement of Natural Rubber by Precipitated Silica: The Influence of Processing Temperature, Fall $182^{\text {nd }}$ Technical Meeting of the Rubber Division, ACS, Cincinnati, Ohio, US, paper no.52, 9-11 October 2012.

10. S.S. Sarkawi, W.K. Dierkes and J.W.M. Noordermeer, Effect of Silane Coupling Agent on the Morphology of Silica-Reinforced Natural Rubber, International Rubber Conference 2013, Paris, France, 20-22 March 2013.

11. S.S. Sarkawi, W.K. Dierkes and J.W.M. Noordermeer, Morphology of Silica-Reinforced Natural Rubber: The Effect of Silane Coupling Agent , Fall $184^{\text {th }}$ Technical Meeting of the Rubber Division, ACS, Cleveland, Ohio, US, paper no.86, 8-10 October 2013. 


\section{Poster Presentation}

12. S.S. Sarkawi, W.K. Dierkes and J.W.M. Noordermeer, Natural Rubber as Renewable Resources for Tires, Impact Energy and Resources Symposium, Enschede, the Netherlands, 14 April 2011 (poster). 


\section{Acknowledgements}

First and foremost, all the praises and thanks to Allah. Alhamdulillah, after three and a half years at the University of Twente, Enschede, the Netherlands I have completed this thesis. During this period, I have received support and help from many people which contribute to this success in their special ways and I really appreciate it.

I wish to express my sincere gratitude to Malaysian Rubber Board (MRB) for giving me the opportunity and financial support to further my post-graduate study. Coming from the country with natural rubber as one of the main commodity, I felt the deep responsibility with this project and hope it will bring benefit to the MRB, Malaysia and society. To all the top management, directors, and all my former heads: Dr. Yusof, Dr. Aris and Che Su, thanks for always seeing the positive in me.

I am especially thankful to my promoter Prof. J.W.M. Noordermeer for accepting me to be his PhD student and be a part of the Elastomer Technology and Engineering group. I am grateful to have the opportunity to work under the renowned scientist. It is an honour to have you as my supervisor. Thank you for your guidance, support and encouragements for me to develop my research skills. You always see the bright side because 'it is all in the game' and as you said 'the easy part has been done before'. For one thing, we do agree that 'NR is so complicated'. I will always remember your motivational phrase 'if you don't have a problem, create one'. You will always inspire me.

As my co-promoter, I would like to thank Dr. Wilma for her advices, fruitful discussions and supports all this years. You have added a feminine touch to the group and things are so much easier when you are around. I also want to express my appreciation to all the members in my graduation committee for their support in completing this thesis. Special thanks also to Dr. Zairossani and Dr. Kannika for valuable comments and suggestions, and to Dr. Victoria and Mark for their help and discussion.

Some work in this thesis was also carried out at Tun Abdul Razak Research Centre (TARRC), Hertford. UK. I would like to acknowledge Dr. Stuart Cook, Dr. Andy Chapman, Dr. Anna Kepas-Suwara, Dr. Robin Davies, Katherine Lawrence, and all staff of TARRC. Thank you for all the supports, help and fruitful discussions. I wish to extend my sincere thanks to all staff of MRB, especially Dayang, Mazlina, Rohaidah and UTGT factory staff, as well as Ahmad Khairul and Aimi Izyana for supplying DPNR and NR serum proteins. 
Studying and living overseas is a challenge and at the same time adventurous. I learned a lot and enjoyed every moment from the kindness and friendship from everyone around me. To all ETE-toppers: Jacob, Ernest, Siti, Anida, Andre, Morteza \& Somayeh, Nadia, Prachid, Karnda, Gerda, Kannika, Yvonne and Ceciel. I had a great time with all of you during the group trips, coffee breaks, lunches and promotion-dinners. My special thanks to Jacob and Anida for being my paranimfen and helping me with the galley proof. My dear cum laude Dr Siti, I really appreciate your friendship and kindness. You always spoiled us with your delicious Thai cookings. To Wisut, the NR-silica team member, thanks for your help in the lab and in the office, as well as the never-ending discussions. Jacob: you sure have a Midas touch in the lab, Ernest: I really appreciate the stimulating discussion on the chem-mystery of silane and silica, Nadia: the lovely cheerful-lady, thanks for the dutch summary and to all dank je well and best of luck!

Not to forget to our Malaysian friends that make our life in Enschede more colourful: Kammarrul \& Rabieah family, Federick and Magritta family, Hakim \& Yana family, Maziah \& Nizam family. To Prof. Yusof and Shahzana, it is a pleasure to know you both and we really appreciate your help and prayers.

Lastly and importantly, I would like to express my sincere gratitude to my family. I owe my deepest thank to my dearest and beloved husband for his love, support and sacrifice all this years. Your love and encouragements is my strength. We had our ups and downs and we learned together to become better person. To my mum \& dad and my dearest sister Nana, thank you for your unconditional love and being supportive. To my late brother, Wan, I miss you and will always pray for you. To all my family, thank you for all your supports to us. 


\section{Curriculum Vitae}

Siti Salina Sarkawi was born in Batu Pahat, Johor, Malaysia on December $17^{\text {th }}, 1976$. She obtained her degree in Chemical Engineering from Vanderbilt University, Nashville, Tennessee, USA in 1998. She joined Malaysian Rubber Board (MRB) in 2000 as a research officer. In 2003, she pursued her MSc in Polymer Materials Science and Engineering at University of Manchester Institute of Science and Technology (UMIST), Manchester, United Kingdom. After completing her MSc in 2004, with a thesis dissertation entitled 'Deformation Micromechanics of Natural Cellulose Fibre', she came back to Malaysia to continue working with MRB as a research officer. She had experience with Malaysian rubber industry in technical advisory and her research work/areas of interest are: natural fillers, epoxidized palm oil, epoxidized natural rubber and silica technology.

In January 2010, she joined ETE Group at University of Twente as a PhD student on the project 'Nano-reinforcement of silica for tire rubber: silica technology for Natural Rubber', under the supervision of Prof. Dr. J.W.M. Noordermeer. At present she is working in Advanced Rubber Technology Unit, MRB at RRIM Research Station Sg.Buloh, Selangor, Malaysia.

Contact address : Siti Salina Sarkawi

Malaysian Rubber Board

Technology \& Engineering Division, RRIM Research Station,

$47000 \mathrm{Sg}$. Buloh,

Selangor,

MALAYSIA

Tel : $+60-(03)-61459400$

Email : ssalina@lgm.gov.my 
"Celik mata, reset minda"

"Oper your eyes and reset your mind"

- Prof. Dr. Murhyg- 